\title{
The complement system and cardiovascular disease
}

Citation for published version (APA):

Hertle, E. (2016). The complement system and cardiovascular disease: the CODAM study. [Doctoral Thesis, Maastricht University]. https://doi.org/10.26481/dis.20160401eh

Document status and date:

Published: 01/01/2016

DOI:

10.26481/dis.20160401eh

Document Version:

Publisher's PDF, also known as Version of record

\section{Please check the document version of this publication:}

- A submitted manuscript is the version of the article upon submission and before peer-review. There can be important differences between the submitted version and the official published version of record.

People interested in the research are advised to contact the author for the final version of the publication, or visit the DOI to the publisher's website.

- The final author version and the galley proof are versions of the publication after peer review.

- The final published version features the final layout of the paper including the volume, issue and page numbers.

Link to publication

\footnotetext{
General rights rights.

- You may freely distribute the URL identifying the publication in the public portal. please follow below link for the End User Agreement:

www.umlib.nl/taverne-license

Take down policy

If you believe that this document breaches copyright please contact us at:

repository@maastrichtuniversity.nl

providing details and we will investigate your claim.
}

Copyright and moral rights for the publications made accessible in the public portal are retained by the authors and/or other copyright owners and it is a condition of accessing publications that users recognise and abide by the legal requirements associated with these

- Users may download and print one copy of any publication from the public portal for the purpose of private study or research.

- You may not further distribute the material or use it for any profit-making activity or commercial gain

If the publication is distributed under the terms of Article $25 \mathrm{fa}$ of the Dutch Copyright Act, indicated by the "Taverne" license above, 
THE COMPLEMENT SYSTEM AND CARDIOVASCULAR DISEASE:

THE CODAM STUDY 
(C) Elisabeth Hertle, Maastricht 2016

Cover design: Jean Scheijen | www.vierdrie.nl

Production: Ipskamp Printing B.V., Enschede

ISBN: 978-94-028-0085-2 


\title{
THE COMPLEMENT SYSTEM AND CARDIOVASCULAR DISEASE:
}

\section{THE CODAM STUDY}

\author{
DISSERTATION
}

to obtain the degree of Doctor at the Maastricht University, on the authority of the Rector Magnificus, Prof. dr. L.L.G. Soete, in accordance with the decision of the Board of Deans, to be defended in public on Friday $1^{\text {st }}$ April 2016, at 14.00 hours

by

Elisabeth Hertle 
Supervisor:

Prof. dr. C.D.A. Stehouwer

Co-supervisors:

Dr. M.M.J. van Greevenbroek

Prof. dr. I.C.W. Arts

Assessment Committee:

Prof. dr. H. ten Cate (chairman)

Prof. dr. N. Marx, University Hospital Aachen, Germany

Prof. dr. M.H. Prins

Dr. J.C. Sluimer

Dr. L.A. Trouw, Leids Universitair Medisch Centrum

The research described in this thesis was supported by a grant of the Dutch Heart Foundation (DHF-NHS2010B194)

Financial support by the Dutch Heart Foundation for the publication of this thesis is gratefully acknowledged.

Financial support from TECOmedical for the printing of this thesis is gratefully acknowledged. 


\section{Contents}

Chapter 1 General introduction 7

Chapter 2 The complement system in human cardiometabolic disease 21

Chapter 3 Complement C3: an emerging risk factor in cardiometabolic 51 disease

Chapter 4 Distinct associations of complement C3a and its precursor C3 with atherosclerosis and cardiovascular disease: The CODAM study

Chapter 5 Complement activation products $\mathrm{C} 5 \mathrm{a}$ and sC5b-9 are associated with low-grade inflammation and endothelial dysfunction, but not with atherosclerosis in a cross-sectional analysis:

The CODAM study

Chapter 6 The alternative complement pathway is longitudinally associated with adverse cardiovascular outcomes: The CODAM study

Chapter 7 Longitudinal associations of factors of the classical complement pathway with cardiovascular outcomes: The CODAM study

Chapter 8 Longitudinal associations of MBL, MASP-1, MASP-2, MASP-3 and MAp44 with cardiovascular outcomes: The CODAM study

Chapter 9 General discussion

Samenvatting 207

Valorisation addendum

Dankwoord

List of publications

Curriculum Vitae 


\section{Chapter 1 CugibfGi I}

General introduction 


\section{Cardiovascular disease}

Cardiovascular disease (CVD) is a major global health issue. According to the World Health Organization (WHO), CVD is the leading cause of death worldwide [1]. Reduction of cardiovascular mortality was also specified as primary global health target in the latest WHO status report on non-communicable diseases 2014 [2].

CVD denotes malfunction of the heart and/or the blood vessels [1]. Coronary heart disease (CHD) constitutes malfunction of the vessels supplying the heart muscle; and cerebrovascular disease constitutes malfunction of the vessels supplying the brain. CHD can culminate in acute myocardial infarction and cerebrovascular disease in stroke, which together represent the most common causes of death from CVD [2]. Peripheral arterial disease (PAD) constitutes malfunction of the vessels supplying the legs and arms, and can entail amputation of the affected limbs [2]. Myocardial infarction, stroke and limb amputation are advanced clinical endpoints of CVD, but actually, malfunction of the blood vessels is a chronic process that starts early in life and progresses subclinically over many years.

\subsection{Aetiology of CVD}

Endothelial dysfunction, atherosclerosis and atherothrombosis are the pathophysiological processes in the arterial vasculature underlying CVD (figure 1). The endothelium is the inner surface of the blood vessels, which serves as barrier and transfers signals from the blood stream into the outer vessel layers and the surrounding tissue, and vice versa [3]. Endothelial dysfunction is considered the first step in the degeneration of blood vessels, and describes the disturbed interaction of the endothelium with the blood stream and the surrounding tissues $[3,4]$. The next stage, atherosclerosis, is characterized by harmful deposition of lipids and immune cells in the vessel wall $[5,6]$. Atherosclerotic deposits (plaque) can be present in such a degree that they narrow the arteries and cause a restriction in blood stream. This results in insufficient oxygen delivery to the surrounding tissue (ischaemia). In ischaemic heart disease, the coronary arteries are narrowed, and the heart function is impaired due to ischaemia in the heart muscle [7]. Plaques can additionally destabilize the vessel and as a result, abrupt ruptures along the plaque and the surrounding vessel surface can occur [8].

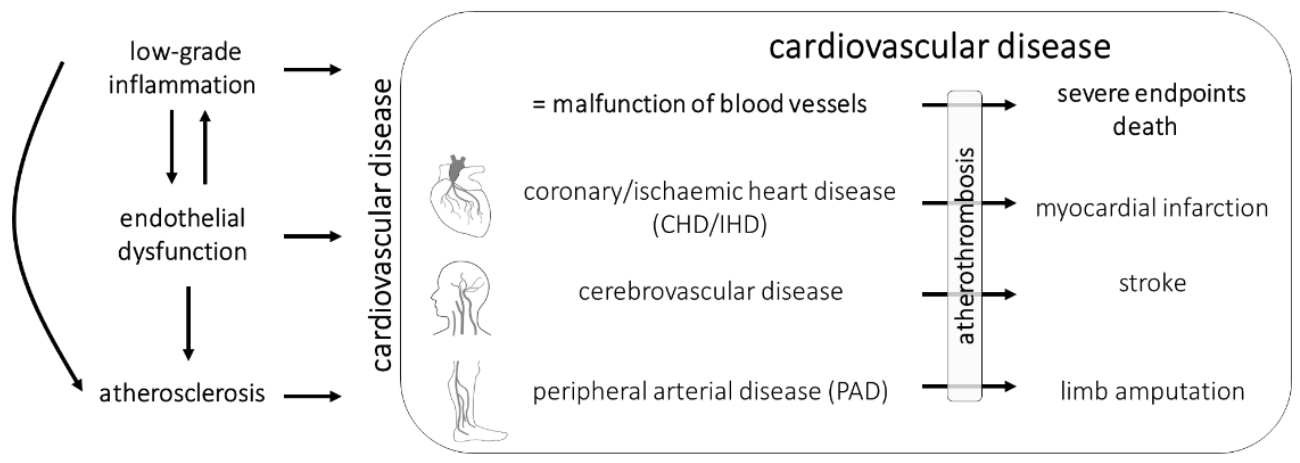

Figure 1: Aetiology, manifestations and endpoints of cardiovascular disease. 
Plaque rupture activates the coagulation system to seal the injured area, but this repair reaction can escalate into excessive clotting and complete arterial occlusion (atherothrombosis). An acute atherothrombotic event leads to prolonged or severe ischaemia, and can thereby cause tissue death. Myocardial infarction and the main form of stroke, ischaemic stroke, entail irreversible destruction of heart or brain tissue as result of tissue death [9].

\subsection{Current situation and future challenges in cardiovascular research}

Over the last decades, considerable progress has been made in treatment and also prevention of CVD. As a result, cardiovascular mortality has been continuously decreasing in high-income countries since the widespread use of lipid-modifying, anti-hypertensive and anti-thrombotic medication [10]. However, there are still evident problems that need to be addressed in order to preserve the current state and to potentially further reduce the burden of CVD: First, in Western countries, a large part of patients with CVD would be assigned a relatively low cardiovascular risk based on the current risk prediction strategies [11]. This means that despite efficient control of known cardiovascular risk factors there may be unrecognized, yet crucial processes contributing to CVD. Second, CVD is a comorbidity of several metabolic and degenerative diseases, such as diabetes mellitus, fatty liver disease and rheumatoid arthritis [12-15]. Furthermore, CVD itself stimulates the development of other degenerative diseases such as Alzheimer's disease [16, 17]. In order to decrease cardiovascular burden not at the expense of other common diseases, treatment of CVD should be improved by intensified targeting of mechanisms that play a role in several chronic diseases. Last, the burden of CVD is at the moment excessively rising in low-income countries [1]. Among other ethnicities, slight variations in aetiology of CVD may exist due to genetic differences and differences in lifestyle $[18,19]$. This suggests that identification of further mechanisms contributing to CVD could potentially have a large impact especially on those societies. Together, these three problems illustrate that there is still considerable need to identify novel risk factors for CVD, despite the accomplishments that have already been made in the past. In particular, better understanding of mechanisms is needed that are not yet included in current risk prediction, that may be involved in several chronic diseases, and that may potentially play a great role in CVD among other ethnicities.

\subsection{Low-grade inflammation and the immune system in CVD}

For these reasons, low-grade inflammation and aberrancies in immune function are of particular interest in cardiovascular research. First, because inflammation and immune dysregulation contribute to several chronic diseases [20]. Next, because the immune system and inflammatory mechanisms have evolved differently among other ethnicities [21, 22]. Furthermore, none of the hitherto established inflammatory markers was consistently shown to add to traditional CVD risk prediction in large studies, which may argue for a continuation of the search for novel biomarkers of CVD risk [23-25].

Inflammation is the body's immediate response to dangers such as tissue damage and infection. The aim of inflammation is to transmit an alarm signal in order to overcome the 
danger and to repair the damage. Inflammatory mediators are powerful signals that induce substantial changes in a broad range of target cells and tissues, and importantly, also in the vasculature. Inflammatory mediators are produced by immune cells, but can also be released from damaged tissues. In chronic low-grade inflammation, however, inflammatory mediators are constantly released at a low level and not as a result of an explicit danger. For example, adipose tissue itself secretes inflammatory mediators in obesity [26]. Lifestyle factors such as smoking and sleep shortage can also lead to chronic release of inflammatory mediators $[27,28]$. Furthermore, chronic, typically Western diseases such as type 2 diabetes mellitus (T2DM) are characterized by chronic inflammation and immune dysregulation [29, 30].

Low-grade inflammation promotes CVD via initiation of endothelial dysfunction, aggravation of atherosclerosis and modulating thrombosis (figure 1) [31-34]. Immune dysregulation incites systemic low-grade inflammation and also directly contributes to cardiovascular damage [35]. Immune cells are characteristic constituents of atherosclerotic plaques and release humoral immune factors locally inside the vessels wall, such as antibodies or matrix metalloproteinases [35]. Thereby, immune cells advance atherosclerosis and contribute to the destabilization of plaques. In addition, humoral immune factors can damage endothelial cells and can activate the coagulation system. One major component of humoral immunity is the complement system. Complement is part of the innate immune system and is an important inducer of inflammation; and is therefore thought to contribute to several processes underlying CVD.

\section{The complement system and CVD}

\subsection{The complement system}

The complement system is a complex protein network. Complement factors are produced by immune cells, in liver, in adipose tissue and by the endothelium. Complement proteins are present in body fluids and tissues, either in soluble form or bound to membranes of circulating or tissue-embedded cells. Complement proteins represent a substantial fraction of plasma proteins and add up to a concentration of $\approx 3 \mathrm{~g} / \mathrm{L}$ in plasma [36]. Complement proteins rank among the first identified components of the immune system and were discovered as early as in the 1890s. Back then, these proteins were observed to aid the killing of bacteria, and were therefore termed 'complement' [37]. Hereafter, many more and also autonomous functions of complement were discovered [38].

Pathways of complement activation [38,39] The complement system consists of more than 30 proteins. Their nomenclature follows the chronological order of their discovery. Most complement factors are inactive proteases that are sequentially activated in a well-organized cascade. Complement is activated by several pattern recognition molecules that can recognize distinct molecular structures. The cascade is subclassified in four complement pathways, which each comprises a series of related enzymatic steps (figure 3 ). The three traditional activation pathways - the 'classical, the lectin and the alternative pathway' - are activated by specific molecular triggers or in a specific microenvironment. Besides, 
complement can be activated by proteases of for example the coagulation and the fibrinolysis system, or by other proteases secreted by immune cells. These miscellaneous activation mechanisms beyond the traditional pathways are gathered in the 'extrinsic pathway'. All activation pathways share a common end route that is started with activation of the central protease C3. Activation of C3 can then initiate the 'terminal pathway'. Besides, particular reactions of the extrinsic pathway can directly activate the terminal pathway.

Function of the complement system Primarily, complement identifies and controls external but also internal dangers, such as damaged, abnormal or obsolete structures. Upon recognition of danger signals, complement is activated to generate an appropriate, fine-tuned immune response. This is achieved by its abilities to selectively recognize diverse molecular structures, to quickly react to them directly, to interact with all other major components of the immune system and to signal to further housekeeping systems. For example, complement interacts with the coagulation system, affects the function of metabolic organs and regulates developmental processes. Thereby, complement modulates processes needed to maintain an immune response and directs tissue regeneration and healing [40-42]. Due to these effects that go far beyond a direct defence reaction, complement is nowadays thought to play a role in multiple physiologic as well as pathophysiologic processes. For example, complement has been implicated in adipose tissue inflammation, insulin resistance, T2DM, fatty liver disease and the metabolic syndrome (detailed overview in chapter 2). Furthermore, complement is thought to be involved in CVD (detailed overview in chapters 2 and 3 ).

\subsection{The complement system in different aetiological processes of CVD}

Complement may be harmful but may also have beneficial functions in the development of CVD (see text box) [43-45]. Because the different complement activation pathways entail characteristic molecular effects, they are each thought to play different roles in the diverse processes underlying CVD. The classical and the lectin pathway are activated by pattern recognition molecules such as $\mathrm{C} 1 \mathrm{q}$ and mannose-binding lectin (MBL) [46]. C1q and MBL can recognize damaged tissues and structures within atherosclerotic deposits, such as apoptotic cells and cholesterol crystals. Experimental studies suggested that the classical and the lectin pathway may be necessary for clear-up processes inside the vessel wall and may therefore be beneficial for vascular health $[43,47]$. In contrast, the alternative pathway is activated spontaneously, and spontaneous activation in the (micro-)vasculature is thought to promote (micro-)vascular damage $[48,49]$. Activation of the terminal pathway immediately generates powerful complement mediators,
Harmful effects of complement in CVD
- release of inflammatory mediators
- activation of endothelial cells
- direct endothelial damage
- activation of immune cells
- smooth muscle cell proliferation
- stimulation of coagulation
- hypofibrinolysis
Beneficial effects of complement in CVD
- clearance of apoptotic/necrotic cells
- clearance of cholesterol deposits
- clearance of infectious triggers for atherosclerosis (Chlamydia pneumoniae) 
and is therefore also thought to contribute to vascular damage [50]. The extrinsic pathway is thought to be involved in thrombosis based on the interaction between complement and coagulation [51-54].

\subsection{Molecular effects of complement activation and suggested roles in CVD}

Each single enzymatic step in the complement cascade generates functional products. This results in a multiplicity of possible response patterns following complement activation. Roughly, the consequences of complement activation can be classified into four main types of response: opsonization, release of soluble mediators (anaphylatoxins), direct interference with membranes and extrinsic protease activation. These different molecular effects contribute to several processes in the development of CVD (figure 2).

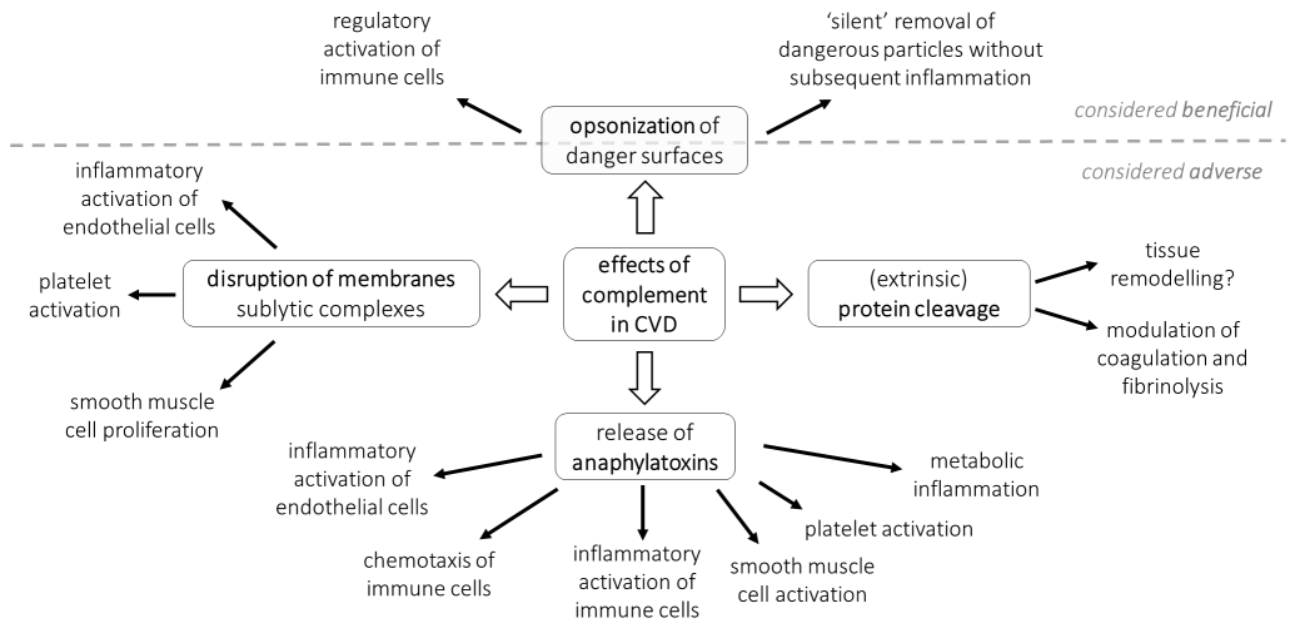

Figure 2: Molecular effects of complement activation and their suggested role in CVD.

Opsonization [39, 43, 45] All successive protease activation steps occur on the surface of the identified danger, while some enzymatic reactions can also occur in the fluid phase. The activated proteases have only a short half-live and are degraded or self-disintegrate rapidly. Proteolytic inactive fragments, such as C3b or C4b, remain however attached to the surface, where they act as specific label (=opsonization). Furthermore, pattern recognition molecules such as C1q and MBL can also act as opsonins by binding to the danger surface without further complement activation. These surface labels can be recognized by manifold receptors on immune and other cells (=opsonin receptors). Opsonin receptors can either be specific for a certain complement fragment, can recognize several complement fragments, or can even recognize various surface modifications including non-complement labels. In general, opsonization without downstream complement activation is considered beneficial, as this can induce 'silent' clear-up processes without subsequent inflammation. In animal studies, transgenic mice lacking $\mathrm{C} 1 \mathrm{q}$ or MBL had more atherosclerosis, which suggested that these 
pattern recognition receptors play a role in the 'silent' removal of deposits inside atherosclerotic lesions [55-57].

Anaphylatoxins [58, 59] Next to these locally confined effects on the surface of dangerous particles, complement activation can result in the release of anaphylatoxins. Anaphylatoxins such as $\mathrm{C} 3 \mathrm{a}, \mathrm{C} 4 \mathrm{a}$ and $\mathrm{C} 5 \mathrm{a}$ are small peptide fragments that are cleaved off from the inactive proteases ( $\mathrm{C} 3, \mathrm{C} 4, \mathrm{C} 5$, respectively) during the activation step. These peptides can spread a signal not only into the local environment but also into the surrounding tissue and even into systemic circulation. Similarly to hormones, anaphylatoxins exert biological effects via binding to specific receptors present in basically all tissues and organs. Currently, there are three known anaphylatoxin receptors: two receptors are well-described, G-protein coupled receptors (C3aR, C5aR1), while the function of the third receptor (C5aR2) is still poorly understood. Notably, for the anaphylatoxin C4a no receptor is identified yet. Anaphylatoxins are strong inflammatory mediators that can promote local as well as systemic inflammation. The designation 'anaphylatoxins' is based on their capacity to induce anaphylactic shock when released in large amounts. Anaphylatoxins are thought to contribute to CVD because they can activate endothelial cells, immune cells, smooth muscle cells and platelets. Anaphylatoxin receptors $\mathrm{C} 3 \mathrm{aR}$ and $\mathrm{C} 5 \mathrm{aR} 1$ were shown to be present in human coronary plaques [60], which implicates C3a and C5a in human CVD. Animal studies suggested a causal role of signalling via $\mathrm{C} 3 \mathrm{a}$, as transgenic mice lacking $\mathrm{C} 3 \mathrm{aR}$ were protected from atherosclerosis $[61,62]$. Furthermore, C3a and C5a were in mice or rats implicated in dietinduced obesity, adipose tissue inflammation and insulin resistance $[63,64]$. This suggested that anaphylatoxins may be involved CVD not only via direct effects on the vasculature but also via induction of inflammation and metabolic disease in other tissues.

Membrane disruption [65] The most direct result of complement activation is the disruption of membranes by insertion of complement proteins into the membrane of a target cell. In contrast to opsonization, which happens on the membrane surface, membrane disruption happens only upon terminal pathway activation. On the target membrane, terminal complement factors C5b, C6, C7, C8, C9 can assemble complexes of different sizes with distinct cellular effects (C5b6, C5b-7, C5b-8, C5b-9). Terminal complement complexes range from small aggregates, which slightly alter membrane integrity, to large lytic pores, which can cause immediate cell death. The induction of cell lysis via formation of large pores, also known as membrane-attack complexes, has long been considered as ultimate result of complement attack. However, induction of cell death is most relevant in the defence against pathogens but is probably of minor relevance in the role of complement in non-infectious processes [38, 39]. Here, terminal complement activation is thought to have biological effects via insertion of smaller aggregates that do not lead to cell lysis but alter membrane organisation and thereby induce intracellular signalling. These sublytic terminal complement complexes were shown to be involved in endothelial activation, smooth muscle cell activation, vascular permeability and platelet activation $[66,67]$. In rabbits, lack of C6, resulting in the inability to form terminal complement complexes, was shown to decrease the severity of atherosclerosis [68]. Furthermore, terminal complement complexes were 
found especially in the deeper layers of human atherosclerotic plaques, which suggested a role of terminal pathway activation in the maturation of atherosclerotic lesions [44].

Extrinsic protein cleavage [69-71] Some active complement proteases may also cleave noncomplement proteins. The last years, a number of non-complement substrates of MASP-1, MASP-2 and MASP-3 were discovered, and their number is likely to increase in the future. Most of the hitherto identified non-complement substrates are part of other protease networks, such as the coagulation, the fibrinolysis or the kinin-kallikrein system. Furthermore, one study suggested a potential function of the complement protease Factor $D$ in tissue remodelling by cleaving structural proteins such as elastin [72].

Complement regulation [37-39] The complement system includes a variety of complement regulators in order to fine-tune its effects. Regulators either influence the kinetic of the proteolytic reactions or inactivate proteases. At each step of the cascade, specific regulators can prevent downstream activation, can inhibit the generation of anaphylatoxins or can prevent the opsonization of surfaces. Healthy host cells express a set of complement regulators on their surface to protect themselves from complement attack. Together, the presence of regulators in the respective microenvironment determines both the extent and the endpoints of complement activation. In general, the presence or absence of complement regulators may be more decisive for the ultimate outcome than the extent of complement activation. Transgenic mice that lacked regulators protecting against terminal complement attack had consistently more severe atherosclerosis in several studies [73-76].

\section{Current gaps in knowledge and aim of the thesis}

As described above, a large body of experimental studies has identified various mechanisms that implicate a causal role of the complement system in CVD. However, knowledge from human studies on complement factors and development of CVD is limited (detailed overview in chapter 2). Several large studies investigated the central protease C3 and the pattern recognition molecule $\mathrm{MBL}$. Most but not all studies on $\mathrm{MBL}$ showed an inverse association of $\mathrm{MBL}$ with future CVD, while observations on $\mathrm{C3}$ were inconsistent or were difficult to compare. However, it has not yet been evaluated whether C3 and MBL are associated with inflammation, endothelial dysfunction and/or atherosclerosis. Furthermore, other complement factors have so far only scarcely been investigated. These studies were mostly small, cross-sectional or included selected patients with pre-existing disease. Most importantly, there is no study that measured simultaneously factors of each complement activation pathway to characterize the role of the different pathways in CVD.

For a more comprehensive appraisal of complement in CVD, this thesis aimed to investigate the different pathways of complement activation within one cohort that is suitable to distinguish different aetiological aspects of CVD. First, this allows evaluating, which part of the complement system is most crucial in development of human CVD. Furthermore, this gives an insight into the relations of complement factors with low-grade inflammation, endothelial dysfunction and atherosclerosis in order to substantiate the evidence from experimental studies in humans. For these reasons, we investigated factors of the different 
complement activation pathways in the CODAM study - a prospective cohort of middle-aged to elderly individuals with an increased cardiometabolic risk.

\subsection{The CODAM cohort}

CODAM is an ongoing cohort study that was designed to investigate the development of metabolic dysfunction and cardiovascular complications (CODAM, cohort study on diabetes and atherosclerosis Maastricht). In order to obtain a wide range in the distribution of cardiometabolic outcomes as well as a relatively high incidence of CVD during the follow-up period, CODAM includes individuals with an increased cardiometabolic risk.

This was achieved as follows: Participants were invited from a large population-based cohort study, the MORGEN study, to participate in the screening if they met the following criteria: living in the area of Maastricht, Caucasian descent, age $>40$ years, and additionally at least one of the following characteristics: $\mathrm{BMI}>25 \mathrm{~kg} / \mathrm{m}^{2}$; use of anti-hypertensive medication; positive family history of T2DM; postprandial blood glucose level $>6.0 \mathrm{mmol} / \mathrm{L}$; history of gestational diabetes and/or glycosuria $[77,78]$. 6108 subjects met these criteria and were invited to undergo the screening, which involved a standard oral glucose tolerance test (OGTT). Of those, 2715 individuals participated in the screening and were diagnosed with normal glucose metabolism (NGM), impaired glucose metabolism (IGM, combining impaired glucose tolerance and impaired fasting glucose) and T2DM according to the WHO criteria of 1999 [79]. All those screened with T2DM ( $n=226)$ and a random selection of those with NGM $(n=728)$ were invited to participate in CODAM. From those screened with IGM, approximately the half were invited to participate in CODAM $(n=291)$, and the other half was allocated to a lifestyle-intervention study (SLIM; study on lifestyle-intervention and impaired glucose tolerance Maastricht). From those invited, 525 individuals were enrolled in CODAM (301 with NGM, 127 with IGM, 97 with T2DM). In order to increase the number of participants with T2DM, 134 patients with known T2DM were in an additional step invited from the original MORGEN cohort, of whom 49 participated. In the end, 574 individuals were included in CODAM (42\% participation rate, 301 with NGM, 127 with IGM, 146 with T2DM).

The baseline examination took place between 1999 and 2001 (CODAM1), and the follow-up examination between 2006 and 2009 (CODAM2) at the university's metabolic research unit. 495 individuals participated in the follow-up examination (14\% attrition rate) with a median follow-up time of 7.0 years [interquartile range: 6.9-7.1 years]. From the 79 subjects lost to follow-up, 37 had died and 42 did not participate for various reasons.

Both at baseline and at follow-up, participants were extensively phenotyped. Data on disease history, smoking, diet, physical activity, health status, parity status (in women) and others were collected by self-administered questionnaires. Adiposity, blood pressure and vascular measurements (carotid artery ultrasound and ankle-brachial index as markers of atherosclerosis), and a 12-lead electrocardiogram (ECG) were performed by a trained research assistant. A second OGTT was performed and venous blood samples were drawn by a study nurse or medical students. In these blood samples, a broad array of metabolic parameters such as lipids and indices of glucose metabolism as well as biomarkers of low- 
grade inflammation and endothelial dysfunction were measured. Furthermore, several common polymorphisms were evaluated with a genome-wide assay.

At baseline, the presence of CVD was assessed by applying a broad definition of CVD in order to have a reference population that was truly free of CVD. At follow-up, presence of CVD and additionally of cardiovascular events (CVE) was assessed in order to distinguish the development of stable CVD from the development of severe CVE. The definition of CVD included the occurrence of at least one of the following: myocardial infarction, stroke, coronary bypass or percutaneous coronary intervention reported by questionnaires; signs of myocardial infarction or coronary ischaemia on an ECG (Minnesota codes 1-1, 1-2, 1-3, 4-1, $4-2,4-3,5-1,5-2,5-3$ or $7-1$ ); non-traumatic amputation or an ankle-brachial index $<0.9$. The ECG measurements were included to identify silent myocardial infarction, which occurs frequently in patients with T2DM [80]. Furthermore, the ECG measurements allowed identifying coronary ischaemia, a less severe stage of CHD. The definition of CVD thus includes cases with overt clinical events, cases with silent events as well as cases with ischaemic disease who did not experience an acute event. In contrast, CVE included only cases with events and was defined as occurrence of at least one of the following: selfreported myocardial infarction, stroke, coronary bypass or percutaneous coronary intervention or signs of myocardial infarction on an ECG (Minnesota codes 1-1, 1-2).

The overall design makes CODAM for several reasons suitable to study the role of complement activation in inflammatory mechanisms of CVD: First of all, the extensive collection of data on several plasma markers of inflammation allows a robust assessment of low-grade inflammation. Elaborate measurement of low-grade inflammation is required for the reliable study of its potential determinants, because low-grade inflammation itself is a relatively vague concept, for which no gold-standard exists. Second, the comprehensive assessment of low-grade inflammation, endothelial dysfunction and atherosclerosis allows investigating the most crucial processes underlying CVD. Distinction of different aetiological processes is needed to gain insight into the mechanisms how complement may contribute to the natural development of CVD. Such knowledge is valuable to appraise the relevance of mechanisms suggested by basic science studies in humans. Furthermore, this also enables to quantify the effect of complement via intermediate variables on CVD. Third, the detailed phenotyping enables the adequate control of potentially confounding cardiometabolic parameters and allows the study of effect modification. Last, the overrepresentation of patients with an elevated cardiovascular risk resulted in an adequate number of events as well as in physiologically relevant changes in intermediate variables during the seven years follow-up. 


\subsection{Thesis outline}

In the current thesis, a set of crucial recognition factors, proteases, activation makers and regulators was measured from each traditional complement pathway, i.e. from the classical, the lectin, the alternative and the terminal pathway (figure 3 ). The associations of C3, C3a, and of terminal pathway factors with cardiovascular outcomes were studied in crosssectional analyses using data of the baseline examination. The associations of classical, lectin and alternative pathway factors with cardiovascular outcomes were studied in longitudinal analyses using data of the baseline and the follow-up examination. Additionally, an overview of previous human studies that investigated complement factors in cardiometabolic disease is provided in chapter 2. Furthermore, mechanisms how C3 and C3 activation may contribute to CVD are discussed in chapter 3.

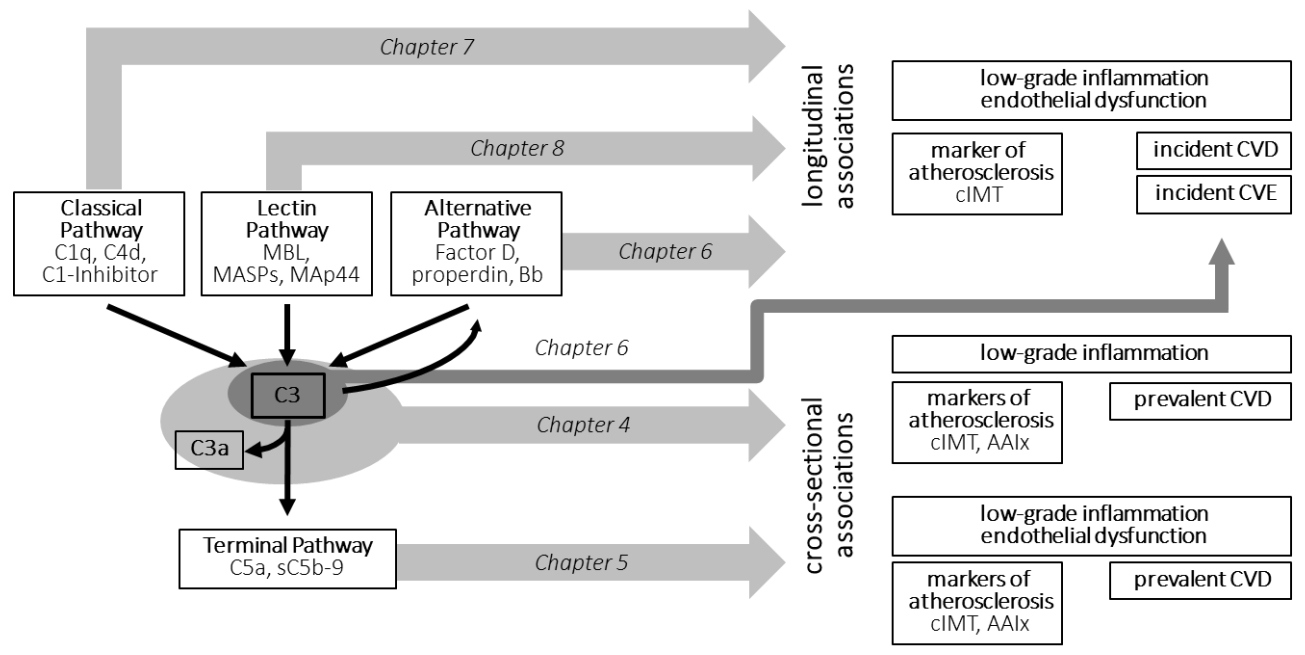

Figure 3: Summary of the investigated associations between the different arms of complement activation and cardiovascular outcomes in the CODAM study. 


\section{References}

1. World Health Organization. Fact sheet $N^{\circ} 317$ 2015. [cited 21 February 2016]; retrieved from http://www.who.int/mediacentre/factsheets/fs317/en/.

2. World Health Organization. Global status report on non-communicable diseases 2014. [cited 21 February 2016]; retrieved from http://apps.who.int/iris/bitstream/10665/148114/1/9789241564854_eng.pdf.

3. Aird WC. Endothelium in health and disease. Pharmacol. Rep., 2008. 60: 139-43.

4. Steyers CM, 3rd, Miller FJ, Jr. Endothelial dysfunction in chronic inflammatory diseases. Int. J. Mol. Sci., 2014. 15: 11324-49.

5. Hansson GK. Inflammation, atherosclerosis, and coronary artery disease. N. Engl. J. Med., 2005. 352: $1685-95$.

6. Hansson GK. Atherosclerosis--an immune disease: The Anitschkov Lecture 2007. Atherosclerosis, 2009. 202: 2-10.

7. American Heart Association, Silent Ischemia and Ischemic Heart Disease 2012. [cited 7 February 2016]; retrieved from http://www.heart.org/HEARTORG/Conditions/HeartAttack/PreventionTreatmentofHeart Attack/Silent-Ischemia-and-Ischemic-Heart-Disease_UCM_434092_Article.jsp.

8. Bentzon JF, Otsuka F, Virmani R, Falk E. Mechanisms of plaque formation and rupture. Circ. Res., 2014. 114: 1852-66.

9. Leys D. Atherothrombosis: a major health burden. Cerebrovasc. Dis., 2001. 11 Suppl 2: 1-4.

10. European Heart Network, European Cardiovascular Disease Statistics 2012. [cited 7 February 2016]; retrieved from http://www.ehnheart.org/cvd-statistics.html.

11. Daviglus ML, Lloyd-Jones DM, Pirzada A. Preventing cardiovascular disease in the 21st century: therapeutic and preventive implications of current evidence. Am. J. Cardiovasc. Drugs, 2006. 6: 87-101.

12. Merkler M, Reiner Z. The burden of hyperlipidaemia and diabetes in cardiovascular diseases. Fundam. Clin. Pharmacol., 2007. 21 Suppl 2: 1-3.

13. Ziegler D. Type 2 diabetes as an inflammatory cardiovascular disorder. Curr. Mol. Med., 2005. 5: 309-22.

14. Symmons DP, Gabriel SE. Epidemiology of CVD in rheumatic disease, with a focus on RA and SLE. Nature reviews. Rheumatology, 2011. 7: 399-408.

15. Armstrong MJ, Adams LA, Canbay A, Syn WK. Extrahepatic complications of nonalcoholic fatty liver disease. Hepatology, 2014. 59: 1174-97.

16. Qiu C, Fratiglioni L. A major role for cardiovascular burden in age-related cognitive decline. Nature reviews. Cardiology, 2015. 12: 267-77.

17. Attems J, Jellinger KA. The overlap between vascular disease and Alzheimer's disease--lessons from pathology. BMC Med., 2014. 12: 206 [doi: 10.1186/s12916-014-0206-2].

18. Dowd JB, Palermo T, Chyu L, Adam E, McDade TW. Race/ethnic and socioeconomic differences in stress and immune function in The National Longitudinal Study of Adolescent Health. Soc. Sci. Med., 2014. 115: 49-55.

19. Wells JC. Ethnic variability in adiposity and cardiovascular risk: the variable disease selection hypothesis. Int. J. Epidemiol., 2009. 38: 63-71.

20. McGeer PL, McGeer EG. Inflammation and the degenerative diseases of aging. Ann. N. Y. Acad. Sci., 2004. 1035: 104-16.

21. Fumagalli M, Sironi M. Human genome variability, natural selection and infectious diseases. Curr. Opin. Immunol., 2014. 30: 9-16.

22. Fumagalli M, Sironi M, Pozzoli U, Ferrer-Admetlla A, Pattini L, Nielsen R. Signatures of environmental genetic adaptation pinpoint pathogens as the main selective pressure through human evolution. PLoS Genet., 2011. 7: e1002355.

23. Krintus M, Kozinski M, Kubica J, Sypniewska G. Critical appraisal of inflammatory markers in cardiovascular risk stratification. Crit. Rev. Clin. Lab. Sci., 2014. 51: 263-79.

24. De Backer G, Graham I, Cooney MT. Do novel biomarkers add to existing scores of total cardiovascular risk? Eur. J. Prev. Cardiol., 2012. 19: 14-7.

25. Ferket BS, van Kempen BJ, Hunink MG, Agarwal I, Kavousi M, Franco OH, Steyerberg EW, Max W, Fleischmann KE. Predictive value of updating Framingham risk scores with novel risk markers in the U.S. general population. PLOS ONE, 2014. 9: e88312.

26. Ouchi N, Parker JL, Lugus JJ, Walsh K. Adipokines in inflammation and metabolic disease. Nat. Rev. Immunol., 2011. 11: 85-97.

27. Shiels MS, Katki HA, Freedman ND, Purdue MP, Wentzensen N, Trabert B, Kitahara CM, Furr M, Li Y, Kemp TJ, Goedert JJ, Chang CM, Engels EA, Caporaso NE, Pinto LA, Hildesheim A, Chaturvedi AK. Cigarette smoking and variations in systemic immune and inflammation markers. J. Natl. Cancer Inst., 2014. 106 [doi: 10.1093/jnci/dju294].

28. Baron KG, Reid KJ. Circadian misalignment and health. Int. Rev. Psychiatry., 2014. 26: 139-54. 
29. Dali-Youcef N, Mecili M, Ricci R, Andres E. Metabolic inflammation: connecting obesity and insulin resistance. Ann. Med., 2013. 45: 242-53.

30. Rao X, Zhong J, Sun Q. The heterogenic properties of monocytes/macrophages and neutrophils in inflammatory response in diabetes. Life Sci., 2014. 116: 59-66.

31. Libby $P$, Okamoto $Y$, Rocha VZ, Folco E. Inflammation in atherosclerosis: transition from theory to practice. Circ. J., 2010. 74: 213-20.

32. Aksu K, Donmez A, Keser G. Inflammation-induced thrombosis: mechanisms, disease associations and management. Curr. Pharm. Des., 2012. 18: 1478-93.

33. Jackson SP. Arterial thrombosis--insidious, unpredictable and deadly. Nat. Med., 2011. 17: 1423-36.

34. Libby P, Hansson GK. Inflammation and immunity in diseases of the arterial tree: players and layers. Circ. Res., 2015. 116: 307-11.

35. Hansson GK, Hermansson A. The immune system in atherosclerosis. Nat. Immunol., 2011. 12: 204-12.

36. Walport MJ. Complement. First of two parts. N. Engl. J. Med., 2001. 344: 1058-66.

37. Sarma JV, Ward PA. The complement system. Cell Tissue Res., 2011. 343: 227-35.

38. Ricklin D, Hajishengallis G, Yang K, Lambris JD. Complement: a key system for immune surveillance and homeostasis. Nat. Immunol., 2010. 11: 785-97.

39. Ehrnthaller C, Ignatius A, Gebhard F, Huber-Lang M. New insights of an old defense system: structure, function, and clinical relevance of the complement system. Mol. Med., 2011. 17: 317-29.

40. Phieler J, Garcia-Martin R, Lambris JD, Chavakis T. The role of the complement system in metabolic organs and metabolic diseases. Semin. Immunol., 2013. 25: 47-53.

41. Markiewski MM, Lambris JD. The role of complement in inflammatory diseases from behind the scenes into the spotlight. Am. J. Pathol., 2007. 171: 715-27.

42. Rutkowski MJ, Sughrue ME, Kane AJ, Ahn BJ, Fang S, Parsa AT. The complement cascade as a mediator of tissue growth and regeneration. Inflamm. Res., 2010. 59: 897-905.

43. Speidl WS, Kastl SP, Huber K, Wojta J. Complement in atherosclerosis: friend or foe? J. Thromb. Haemost., 2011. 9: 428-40.

44. Oksjoki R, Kovanen PT, Meri S, Pentikainen MO. Function and regulation of the complement system in cardiovascular diseases. Front. Biosci., 2007. 12: 4696-708.

45. Szeplaki G, Varga L, Fust G, Prohaszka Z. Role of complement in the pathomechanism of atherosclerotic vascular diseases. Mol. Immunol., 2009. 46: 2784-93.

46. Degn SE, Thiel S. Humoral pattern recognition and the complement system. Scand. J. Immunol., 2013. 78 : 181-93.

47. Bohlson SS, Fraser DA, Tenner AJ. Complement proteins C1q and MBL are pattern recognition molecules that signal immediate and long-term protective immune functions. Mol. Immunol., 2007. 44: 33-43.

48. Thurman JM, Holers VM. The central role of the alternative complement pathway in human disease. J. Immunol., 2006. 176: 1305-10.

49. Zipfel PF, Heinen S, Jozsi M, Skerka C. Complement and diseases: defective alternative pathway control results in kidney and eye diseases. Mol. Immunol., 2006. 43: 97-106.

50. Francescut L, Steiner T, Byrne S, Cianflone K, Francis S, Stover C. The role of complement in the development and manifestation of murine atherogenic inflammation: novel avenues. J. Innate Immun., 2012. 4: 260-72.

51. Weitz IC. Complement the hemostatic system: an intimate relationship. Thromb. Res., 2014. 133 Suppl 2: S117-21.

52. Peerschke EI, Yin W, Ghebrehiwet B. Platelet mediated complement activation. Adv. Exp. Med. Biol., 2008. 632: 81-91.

53. Peerschke El, Yin W, Ghebrehiwet B. Complement activation on platelets: implications for vascular inflammation and thrombosis. Mol. Immunol., 2010. 47: 2170-5.

54. Conway EM. Reincarnation of ancient links between coagulation and complement. J. Thromb. Haemost., 2015. 13 Suppl 1: S121-32.

55. Bhatia VK, Yun S, Leung V, Grimsditch DC, Benson GM, Botto MB, Boyle JJ, Haskard DO. Complement C1q reduces early atherosclerosis in low-density lipoprotein receptor-deficient mice. Am. J. Pathol., 2007. 170: 416-26.

56. Lewis MJ, Malik TH, Ehrenstein MR, Boyle JJ, Botto M, Haskard DO. Immunoglobulin M is required for protection against atherosclerosis in low-density lipoprotein receptor-deficient mice. Circulation, 2009. 120: 417-26.

57. Matthijsen RA, de Winther MP, Kuipers D, van der Made I, Weber C, Herias MV, Gijbels MJ, Buurman WA. Macrophage-specific expression of mannose-binding lectin controls atherosclerosis in low-density lipoprotein receptor-deficient mice. Circulation, 2009. 119: 2188-95. 
58. Klos A, Tenner AJ, Johswich KO, Ager RR, Reis ES, Kohl J. The role of the anaphylatoxins in health and disease. Mol. Immunol., 2009. 46: 2753-66.

59. Zhou W. The new face of anaphylatoxins in immune regulation. Immunobiology, 2012. 217: 225-34.

60. Oksjoki R, Laine P, Helske S, Vehmaan-Kreula P, Mayranpaa MI, Gasque P, Kovanen PT, Pentikainen MO. Receptors for the anaphylatoxins C3a and C5a are expressed in human atherosclerotic coronary plaques. Atherosclerosis, 2007. 195: 90-9.

61. Sakuma M, Morooka T, Wang Y, Shi C, Croce K, Gao H, Strainic M, Medof ME, Simon DI. The intrinsic complement regulator decay-accelerating factor modulates the biological response to vascular injury. Arterioscler. Thromb. Vasc. Biol., 2010. 30: 1196-202.

62. Yang $X$, Peterson L, Thieringer R, Deignan JL, Wang X, Zhu J, Wang S, Zhong H, Stepaniants S, Beaulaurier J, Wang IM, Rosa R, Cumiskey AM, Luo JM, Luo Q, Shah K, Xiao J, Nickle D, Plump A, Schadt EE, Lusis AJ, Lum PY. Identification and validation of genes affecting aortic lesions in mice. J. Clin. Invest., 2010. 120: 2414-22.

63. Mamane Y, Chung Chan C, Lavallee G, Morin N, Xu LJ, Huang J, Gordon R, Thomas W, Lamb J, Schadt EE, Kennedy BP, Mancini JA. The C3a anaphylatoxin receptor is a key mediator of insulin resistance and functions by modulating adipose tissue macrophage infiltration and activation. Diabetes, 2009. 58: 2006-17.

64. Lim J, Iyer A, Suen JY, Seow V, Reid RC, Brown L, Fairlie DP. C5aR and C3aR antagonists each inhibit dietinduced obesity, metabolic dysfunction, and adipocyte and macrophage signaling. FASEB J., 2013. 27: 822-31.

65. Tegla CA, Cudrici C, Patel S, Trippe R, 3rd, Rus V, Niculescu F, Rus H. Membrane attack by complement: the assembly and biology of terminal complement complexes. Immunol. Res., 2011. 51: 45-60.

66. Salama A, Hugo F, Heinrich D, Hoge R, Muller R, Kiefel V, Mueller-Eckhardt C, Bhakdi S. Deposition of terminal C5b-9 complement complexes on erythrocytes and leukocytes during cardiopulmonary bypass. N. Engl. J. Med., 1988. 318: 408-14.

67. Wiedmer T, Esmon CT, Sims PJ. Complement proteins C5b-9 stimulate procoagulant activity through platelet prothrombinase. Blood, 1986. 68: 875-80.

68. Schmiedt W, Kinscherf R, Deigner HP, Kamencic H, Nauen O, Kilo J, Oelert H, Metz J, Bhakdi S. Complement C6 deficiency protects against diet-induced atherosclerosis in rabbits. Arterioscler. Thromb. Vasc. Biol., 1998. 18: $1790-5$.

69. Oikonomopoulou K, Ricklin D, Ward PA, Lambris JD. Interactions between coagulation and complement--their role in inflammation. Semin. Immunopathol., 2012. 34: 151-65.

70. Dobo J, Schroeder V, Jenny L, Cervenak L, Zavodszky P, Gal P. Multiple roles of complement MASP-1 at the interface of innate immune response and coagulation. Mol. Immunol., 2014. 61: 69-78.

71. Lupu F, Keshari RS, Lambris JD, Coggeshall KM. Crosstalk between the coagulation and complement systems in sepsis. Thromb. Res., 2014. 133 Suppl 1: S28-31.

72. Beazley KE, Reckard S, Nurminsky D, Lima F, Nurminskaya M. Two sides of MGP null arterial disease: chondrogenic lesions dependent on transglutaminase 2 and elastin fragmentation associated with induction of adipsin. J. Biol. Chem., 2013. 288: 31400-8.

73. An G, Miwa T, Song WL, Lawson JA, Rader DJ, Zhang Y, Song WC. CD59 but not DAF deficiency accelerates atherosclerosis in female ApoE knockout mice. Mol. Immunol., 2009. 46: 1702-9.

74. Lewis RD, Jackson CL, Morgan BP, Hughes TR. The membrane attack complex of complement drives the progression of atherosclerosis in apolipoprotein E knockout mice. Mol. Immunol., 2010. 47: 1098-105.

75. Wu G, Hu W, Shahsafaei A, Song W, Dobarro M, Sukhova GK, Bronson RR, Shi GP, Rother RP, Halperin JA, Qin $X$. Complement regulator CD59 protects against atherosclerosis by restricting the formation of complement membrane attack complex. Circ. Res., 2009. 104: 550-8.

76. Yun S, Leung VW, Botto M, Boyle JJ, Haskard DO. Brief report: accelerated atherosclerosis in low-density lipoprotein receptor-deficient mice lacking the membrane-bound complement regulator CD59. Arterioscler. Thromb. Vasc. Biol., 2008. 28: 1714-6.

77. Kruijshoop M, Feskens EJ, Blaak EE, de Bruin TW. Validation of capillary glucose measurements to detect glucose intolerance or type 2 diabetes mellitus in the general population. Clin. Chim. Acta, 2004. 341: 33-40.

78. van Dam RM, Boer JM, Feskens EJ, Seidell JC. Parental history of diabetes modifies the association between abdominal adiposity and hyperglycemia. Diabetes Care, 2001. 24: 1454-9.

79. Alberti KG, Zimmet PZ. Definition, diagnosis and classification of diabetes mellitus and its complications. Part 1: diagnosis and classification of diabetes mellitus provisional report of a WHO consultation. Diabet. Med., 1998. 15: 539-53.

80. Arenja N, Mueller C, Ehl NF, Brinkert M, Roost K, Reichlin T, Sou SM, Hochgruber T, Osswald S, Zellweger MJ. Prevalence, extent, and independent predictors of silent myocardial infarction. Am. J. Med., 2013. 126: 515-22. 


\section{Chapter 2 Cusibfi 5}

The complement system in human cardiometabolic disease

Molecular Immunology, 2014. 61: 135-48

E. Hertle, C.D.A. Stehouwer, M.M.J. van Greevenbroek 


\begin{abstract}
The complement system has been implicated in obesity, fatty liver, diabetes and cardiovascular disease (CVD). Complement factors are produced in adipose tissue and appear to be involved in adipose tissue metabolism and local inflammation. Thereby complement links adipose tissue inflammation to systemic metabolic derangements, such as low-grade inflammation, insulin resistance and dyslipidaemia. Furthermore, complement has been implicated in pathophysiological mechanisms of diet- and alcohol induced liver damage, hyperglycaemia, endothelial dysfunction, atherosclerosis and fibrinolysis.

In this review, we summarize current evidence on the role of the complement system in several processes of human cardiometabolic disease. C3 is the central component in complement activation, and has most widely been studied in humans. C3 concentrations are associated with insulin resistance, liver dysfunction, risk of the metabolic syndrome, type 2 diabetes and CVD. C3 can be activated by the classical, the lectin and the alternative pathway of complement activation; and downstream activation of C3 activates the terminal pathway. Complement may also be activated via extrinsic proteases of the coagulation, fibrinolysis and the kinin systems. Studies on the different complement activation pathways in human cardiometabolic disease are limited, but available evidence suggests that they may have distinct roles in processes underlying cardiometabolic disease. The lectin pathway appeared beneficial in some studies on type 2 diabetes and CVD, while factors of the classical and the alternative pathway were related to unfavourable cardiometabolic traits. The terminal complement pathway was also implicated in insulin resistance and liver disease, and appears to have a prominent role in acute and advanced CVD.

The available human data suggest a complex and potentially causal role for the complement system in human cardiometabolic disease. Further, preferably longitudinal studies are needed to disentangle which aspects of the complement system and complement activation affect the different processes in human cardiometabolic disease.
\end{abstract}




\section{Cardiometabolic disease}

Cardiometabolic disease describes a spectrum of interconnected pathobiological alterations in metabolic organs and the cardiovascular system that alone and in concert increase cardiovascular disease burden [1,2]. Our modern lifestyle, with excess energy intake and sedentary behaviour, forms the basis for the current epidemic of overweight and obesity. Central obesity, i.e. the accumulation of fat in and around the abdominal area, is associated with cardiometabolic traits such as dyslipidaemia, insulin resistance, type 2 diabetes (T2DM), and ultimately cardiovascular disease [3].

When adipose tissue $\operatorname{depot}(\mathrm{s})$ are enlarged to a size that exceeds a certain critical mass, adipocytes are presumed to be exposed to hypoxia due to adipocyte hypertrophy and decreased capillary density in the expanding adipose tissue [4]. Hypoxia itself poses a direct cellular stress signal that can induce inflammatory reactions. Additionally, nutrient excess may overcharge adipocyte metabolism and thereby induce endoplasmic reticulum stress, oxidative stress and mitochondrial dysfunction. These adverse conditions in obesity favour the release of proinflammatory adipokines and induce adipocyte apoptosis and necrosis. Macrophages that are directed towards the dying adipocytes also release proinflammatory mediators [5]. This local adipose tissue inflammation is thought to initiate adipose tissue insulin resistance and to spill over into systemic low-grade inflammation [6]. In addition, visceral adipose tissue, which is located around the internal organs, sensitively reacts to stress hormones such as catecholamines and glucocorticoids, leading to lipolysis [7]. Central obesity will thus lead to the combined release of free fatty acids (FFAs) and adipocytokines into the hepatic and systemic circulation. This forms the unfavourable concert that can enhance the further metabolic consequences of central obesity.

The liver is directly exposed to diet-derived nutrient excess and to visceral adipose tissue products [8]. Adipokines, FFAs and nutrient overload can impair normal substrate metabolism in the liver and thereby promote hepatic inflammation and hepatic fat accumulation [9]. Hepatic fat accumulation itself induces hepatocyte dysfunction. Moreover, it can contribute to hepatic insulin resistance via disruption of glucose and lipid metabolism, leading to dyslipidaemia [10]. The liver is a crucial source of many protein species; therefore, production of coagulation, immune and growth factors is also altered in hepatocyte dysfunction. In muscle, lipid overflow from insulin-resistant adipose tissue in combination with a proinflammatory environment and a sedentary lifestyle is thought to cause muscle ectopic fat deposition, which in turn promotes muscle insulin resistance [11]. These multiple metabolic derangements in the main insulin-sensitive organs constitute a state of systemic insulin-resistance and once established, entail further metabolic and cardiovascular impairments.

Insulin resistance is the impaired capacity of insulin-sensitive organs such as muscle, liver, adipose tissue and the vasculature to respond to a given concentration of insulin [6]. In insulin resistance, predominantly the metabolic insulin signalling pathway is affected [3]. In its early stages, insulin resistance can be counteracted by compensatory hyperinsulinaemia, which aggravates metabolic stress and may facilitate the expansion of fat mass. Progressively, insulin resistance results in increased glucose levels both after food intake and 
in the fasting state, and further enhances dyslipidaemia in the liver. Glucotoxicity, lipotoxicity and inflammation are thought to promote beta-cell failure, which may lead to the development of T2DM [12].

The multiple metabolic derangements originating from central adiposity also affect the cardiovascular system. Insulin resistance in the microvasculature increases peripheral vascular resistance, favours the development of hypertension and contributes to systemic insulin resistance by blunting insulin-mediated perfusion and nutrient supply in peripheral tissues [13]. Inflammation, dyslipidaemia and hyperglycaemia initiate endothelial dysfunction and promote atherosclerosis [14]. Impaired regulation of the production of coagulation and fibrinolytic factors by liver and adipose tissue establishes a hypercoagulable, prothrombotic state [15]. Notably, these cardiometabolic impairments aggravate each other in vicious circles. This explains why a disbalance, once established in either adipose tissue, liver or cardiovascular system, facilitates the development of further cardiometabolic impairments. The clustered occurrence of cardiometabolic risk factors is amongst others reflected in the metabolic syndrome (MetS), which combines central adiposity, dyslipidaemia, hyperglycaemia and hypertension [3].

Taken together, there is a strong interrelatedness between various obesity-associated cardiometabolic risk factors. Moreover, there is also great interindividual variability in the response to adiposity, as a great part of obese individuals preserves normal glucose tolerance [16]. One biological system that may participate in the unfavourable response to (central) fat accumulation and may contribute to essential pathophysiological mechanisms in cardiometabolic disease is the complement system. The complement system has long-known connections to several features of cardiometabolic disease, and recent discoveries further corroborate these relations. In this review, we will summarize current evidence on the role of the complement system in several processes of adiposity-associated cardiometabolic disease, with particular focus on the available human data.

\section{The complement system}

The complement system is a complex protein network of the innate immune system. It consists of soluble and membrane-bound proteins functioning in cascades of stepwise protease activation [17]. Complement can be activated by three major pathways, the classical pathway, the lectin pathway and the alternative pathway [18]. Activation of any of the three pathways can lead to the cleavage of $\mathrm{C} 3$, and subsequent activation of $\mathrm{C} 5, \mathrm{C} 6, \mathrm{C} 7$, $\mathrm{C} 8$ and $\mathrm{C} 9$ of the terminal pathway. $\mathrm{C} 1 \mathrm{q}$ and $\mathrm{MBL}$ are pattern recognition molecules of the classical and the lectin pathway, respectively; and C1s, C1r, MBL-associated serine proteases (MASP), C2 and C4 further participate in classical and lectin pathway activation of C3 [19]. The alternative pathway activates $\mathrm{C} 3$ spontaneously in combination with Factor B (FB), Factor $D(F D)$ and properdin [20]. Furthermore, activation of C3 and C5 via extrinsic proteases of the coagulation, fibrinolysis and the kinin systems has nowadays been recognized as a fourth complement activation pathway [21].

During complement activation, the anaphylatoxins $\mathrm{C} 3 \mathrm{a}$ and $\mathrm{C} 5 \mathrm{a}$ are released, but also multiple other protein fragments are produced, such as C4d, Bb, C3c and C3d [22, 23]. 
Completion of the terminal pathway results in the formation of membrane-bound and soluble C5b-9 complexes (C5b-9, also known as membrane-attack complex and sC5b-9, respectively) [24]. There are multiple complement inhibitors that can regulate the degree of complement activation [18]. C1-Inhibitor (C1-INH) and C4b-binding protein (C4BP) are regulators of the classical and the lectin pathway, while Factor $\mathrm{H}(\mathrm{FH})$, factor I (FI) and properdin are regulators of the alternative pathway. Complement can trigger responses via binding of anaphylatoxins to their receptors (C3a-receptor (C3aR), C5a-receptors (C5aR1 and C5aR2)) but also via surface bound fragments that can be recognized by other cellular receptors such as complement receptors (CR) 1-4 [22, 25]. Protein complexes of the terminal pathway insert into cell membranes and thereby induce intracellular signals or disturb membrane integrity, eventually promoting cell lysis [24]. Complement is ubiquitously present, and human cells express a variety of complement regulators to protect themselves from complement attack. The body has to maintain a delicate balance between complement activation and inhibition, as unbalanced complement control can result in immune dysregulation and tissue damage [26]. Consequent on its role in multiple processes in various organs, the complement system may contribute to the development and progression of cardiometabolic disease.

\section{The complement system in adipose tissue}

Adipose tissue is considered a metabolically active immune organ [27]. It is also a source and a target of many complement factors. Human adipose tissue produces and secretes many factors of the classical, lectin, alternative and terminal pathways (table 1). In vivo, both adipose and non-adipose cells (such as endothelial cells or macrophages) are likely to contribute to adipose tissue complement production, but studies using isolated adipocytes have shown that at least $\mathrm{C} 1 \mathrm{q}, \mathrm{C} 1 \mathrm{r}, \mathrm{C} 1 \mathrm{~s}, \mathrm{C2}, \mathrm{C} 3, \mathrm{C} 4, \mathrm{C} 6, \mathrm{C} 7, \mathrm{FD}, \mathrm{FB}, \mathrm{FH}$ are actually expressed in the adipocyte itself (table 1). In observational studies, plasma levels of C3, C3a, FB, FD and $\mathrm{FH}$ were associated with body mass index (BMI) [28] or were higher in obese compared to non-obese subjects [29-33]. Moreover, C3, FB, and FH in obese patients were shown to decrease after weight loss [34-37], and low levels of C3, FB, FD and FH in patients with anorexia nervosa increased upon weight gain [35]. In addition, plasma C3 was, in two human proteomics studies, identified as key marker for differences in body fat and body fat changes $[38,39]$. Taken together, these data suggests that adipose tissue is a relevant determinant of at least local, but potentially also systemic, levels of these complement factors.

Complement was not only shown to be associated with the degree of obesity, but also with qualitative measures of obesity such as body fat distribution. Circulating concentrations of C3 and FD were associated with waist circumference and/or waist-hip ratio, which are both measures of central obesity [40-42], and C3 was independently of BMI associated with central adiposity [43]. A potential role for complement in visceral adipose tissue is further corroborated by studies in human adipose tissue, which have shown higher expression of FB in visceral, as compared to subcutaneous adipose tissue [44-46]. Also C1q, C1r, C1s, C2, C4, C7 were found to be more highly expressed in visceral compared to subcutaneous adipose tissue [44], while results were inconsistent for C3 and FD [44, 46-48]. 
Systemic metabolic derangements may affect complement expression in adipose tissue (or vice versa), as an increased expression of $\mathrm{C} 1 \mathrm{r}, \mathrm{C} 1 \mathrm{~s}, \mathrm{C} 3$ and $\mathrm{C} 7$ was found in adipose tissue from individuals with insulin resistance or T2DM [49-52]. Both in dyslipidaemic individuals and in controls, adipose tissue expression of C3 was associated with an impaired response to insulin and with glucose intolerance as determined by oral glucose tolerance test [53]. We recently showed that adipocyte expression of the complement system as a whole was associated with higher plasma lipids and also larger waist circumference [54]. In this study, adipocyte complement expression also correlated with proxy measures of adipose tissue insulin resistance. We additionally showed that the association of body fat and fat distribution with systemic C3 concentrations was, for a large part, explained by systemic inflammation and insulin resistance [55].

Complement is not only produced, but also activated in human adipose tissue. Interestingly, higher levels of activated C3 (C3d) were found in adipose tissue of obese, insulin resistant as compared to lean women [56]. The expression of two complement anaphylatoxin receptors i.e. C3AR and C5AR1 in adipocytes (table 1) further presents adipose tissue as target of complement activation. With use of knock-out mouse models, both the C5aR1 [57] and the C3aR [58] were shown to be instrumental in obesity-induced metabolic derangements. In mice fed a high-fat diet, absence of either anaphylatoxin receptor was generally associated with less adipose tissue inflammation and preserved insulin sensitivity. In addition, complement is increasingly recognized to exert metabolic effects in adipose tissue, where especially C3 activation via the alternative pathway appears to mediate postprandial lipid uptake and lipid metabolism in adipocytes $[59,60]$. In fact, C3a-desArg, which is also known as acylation stimulating protein (ASP), has been discussed over the past two decades to be crucially involved in adipocyte lipid metabolism [61, 62], and recently, studies in transgenic mice also implicated FB and properdin in postprandial lipid uptake in adipose tissue $[63,64]$. These observations suggest complement as mediator of adipose tissue sensitivity to dietary overload. Remarkably, in a large human study in healthy men, plasma C3 concentration was reported to predict weight gain over a 6-year period independently of other lifestyle or metabolic factors [65]. In contrast to the data presented above, the lectin pathway does not appear have a clear role in adipose tissue. MBL has not been detected in adipose tissue, and was in larger human studies not correlated with BMI or affected by weight loss $[66,67]$. On the other hand, MASP-2, MASP-3 and MAp44 were correlated with BMI in some studies [68, 69], which deserves further investigation.

Taken together, an array of complement factors, mainly of the classical, the alternative and the terminal complement pathway, are produced in adipose tissue, appear to be involved in both adipose tissue inflammation and adipose tissue metabolism, and might be even be causally involved in the progression of obesity. The complement system might thus link adipose tissue inflammation to systemic metabolic derangements. 
Table 1: Summary of current data on the expression and production of the main components of the complement system in human adipose tissue ${ }^{a}$

\begin{tabular}{|c|c|c|c|c|c|}
\hline Name & GeneSymbol & Source $(s)^{b}$ & $\operatorname{Depot}^{c}$ & $\begin{array}{l}\text { RNA or } \\
\text { protein }{ }^{d}\end{array}$ & References $^{\mathrm{e}}$ \\
\hline \multicolumn{6}{|c|}{ Classical pathway } \\
\hline $\mathrm{C} 1 \mathrm{q}$ & $C 1 Q A / B / C$ & AT, ADIP, AS & $\mathrm{O}-\mathrm{V}, \mathrm{SC}, \mathrm{PCA}$ & RNA, P & $A, B, F, P, S, V$ \\
\hline C1s & C1S & AT, ADIP, AS & $\mathrm{O}-\mathrm{V}, \mathrm{SC}, \mathrm{PCA}$ & RNA, $P$ & $A, B, C, L, N, P, R, V$ \\
\hline C1r & $C 1 R$ & AT, ADIP, AS & $\mathrm{O}-\mathrm{V}, \mathrm{SC}, \mathrm{PCA}$ & RNA, $P$ & $A, B, M, N, P, R, S, V$ \\
\hline C2 & $C 2$ & $\mathrm{AT}, \mathrm{AS}$ & $\mathrm{O}-\mathrm{V}, \mathrm{SC}, \mathrm{PCA}$ & RNA, $P$ & $A, F, L, P, S, V$ \\
\hline C4 & $C 4 A / B$ & AT, AS & $\mathrm{O}-\mathrm{V}, \mathrm{SC}, \mathrm{PCA}$ & RNA, $P$ & $A, L, P, V$ \\
\hline \multicolumn{6}{|c|}{ Lectin Pathway } \\
\hline MASP-1 & MASP1 & AT & O-V, SC & RNA & K \\
\hline \multicolumn{6}{|c|}{ Alternative Pathway } \\
\hline C3 & C3 & AT, ADIP, AS & $\mathrm{O}-\mathrm{V}, \mathrm{SC}, \mathrm{PCA}$ & RNA, $P$ & $\begin{array}{l}A, B, C, D, E, G, J, L, M, P, Q, \\
T, \cup, V\end{array}$ \\
\hline FB & $C F B$ & AT, ADIP, AS & $\mathrm{O}-\mathrm{V}, \mathrm{SC}, \mathrm{PCA}$ & RNA, $P$ & $A, G, H, L, P, S, T, V$ \\
\hline FD & CFD & $A T, A D I P, A S$ & $\mathrm{O}-\mathrm{V}, \mathrm{SC}, \mathrm{PCA}$ & RNA, $P$ & $A, B, G, I, L, M, N, P, T, V$ \\
\hline \multicolumn{6}{|c|}{ Terminal Pathway } \\
\hline C5 & C5 & AT & O-V, SC & RNA & $\mathrm{T}$ \\
\hline C6 & C6 & ADIP & SC & RNA & B \\
\hline C7 & $C 7$ & $A T, A D I P, A S$ & $\mathrm{O}-\mathrm{V}, \mathrm{SC}, \mathrm{PCA}$ & RNA, $P$ & $A, B, C, F, M, P, V$ \\
\hline \multicolumn{6}{|c|}{ Regulatory proteins } \\
\hline C1-INH & SERPING1 & ADIP, AS & SC, PCA & RNA, P & $B, M, N, O, P$ \\
\hline $\mathrm{FH}$ & $\mathrm{CFH}$ & AT, ADIP, AS & O-V, SC, PCA & RNA, $P$ & $B, H, I, M, P, T, V$ \\
\hline $\mathrm{FI}$ & $C F I$ & AT & O-V, SC & RNA & $\mathrm{T}$ \\
\hline $\mathrm{Cn}$ & $C L U$ & AT, ADIP, AS & $\mathrm{O}-\mathrm{V}, \mathrm{SC}, \mathrm{PCA}$, & RNA, P & $B, L, N, P, S, V$ \\
\hline DAF & CD55 & ADIP & SC & RNA & B \\
\hline CD59 & CD59 & ADIP & SC, & RNA & B \\
\hline \multicolumn{6}{|c|}{ Receptors } \\
\hline gC1qR & $C 1 q B P$ & ADIP, AS & SC, PCA & RNA, P & $\mathrm{B}, \mathrm{N}$ \\
\hline $\mathrm{cC} 1 \mathrm{qR}$ & $C A L R$ & ADIP, AS & SC, PCA & RNA, $P$ & $B, N$ \\
\hline C3aR & C3AR1 & AT, ADIP & $\mathrm{O}-\mathrm{V}, \mathrm{SC}$ & RNA & $\mathrm{B}, \mathrm{S}, \mathrm{T}, \mathrm{U}$ \\
\hline C5aR1 & C5AR1 & AT, ADIP & O-V, SC & RNA & $B, T$ \\
\hline C5aR2 & C5AR2 & AT & O-V, SC & RNA & $\mathrm{T}$ \\
\hline CR1 & CR1 & AT & O-V, SC & RNA & $\mathrm{F}, \mathrm{T}$ \\
\hline CR3 & ITGAM & ADIP & SC & RNA & B \\
\hline CR4 & ITGAX & ADIP & SC & RNA & B \\
\hline
\end{tabular}

${ }^{a}$ the following factors were specifically searched for, but have not been reported to be expressed in human adipose tissue: MBL, ficolins, MASP-2, C8, C9, C4BP, properdin, Vn, MCP, CD93, CR2

${ }^{\text {b } A T ;}$; adipose tissue (stromal vascular cells \& adipocytes), ADIP; isolated adipocytes, AS; adipocyte or AT secretome (not necessarily within 1 publication)

c O-V; omental or visceral fat, SC; subcutaneous fat, PCA; primary cultured adipocytes, i.e. differentiated stromal vascular cells (not necessarily within 1 publication)

${ }^{d}$ not necessarily within 1 publication

e A; [44], B; [54], C; [49], D; [47], E; [50], F; [70], G; [46], H; [45], I; [48], J; [53], K; [71], L; [72], M; [73], N; [74], O; [75], P; [30], Q; [52], R; [51], S; [76], T; [77], U; [78], V, [79] 


\section{The complement system in low-grade inflammation and insulin resistance}

The above-described relations between complement and adiposity strongly suggest a role for the complement system also in systemic inflammation and systemic insulin resistance. In large studies ( $\mathrm{N}>1000), \mathrm{C} 3, \mathrm{C4}, \mathrm{MBL}$ and FD correlated with plasma C-reactive protein (CRP) or with other inflammatory markers [80-83]. Furthermore, plasma $\mathrm{C} 1 \mathrm{q}, \mathrm{FB}, \mathrm{FH}$, properdin, C1-INH and C4BP correlated with CRP in smaller studies $(\mathrm{N}<300)$ [84-87]. We additionally showed that $\mathrm{C} 3, \mathrm{C} 3 \mathrm{a}, \mathrm{C} 5 \mathrm{a}$ and $\mathrm{SC} 5 \mathrm{~b}-9$ were, independently of confounding factors, associated with systemic low-grade inflammation [88, 89].

Insulin resistance is, in human studies, usually assessed by euglycaemic-hyperinsulinaemic clamp, HOMA index or fasting insulin. The clamp technique is considered to be the gold standard but is labour-intensive and often not available in large epidemiological studies. HOMA, which is derived from fasting glucose and fasting insulin, has been validated against the euglycaemic-hyperinsulinaemic clamp [90], while fasting insulin reflects hyperinsulinaemia, i.e. the compensatory response to insulin resistance. In unadjusted analyses, C3 was correlated with HOMA in populations of different age, health and ethnicity [80, 91-94]. The association of C3 with HOMA was shown to be independent of confounding factors among a broad range of individuals, including healthy adults, overweight and obese, elderly and patients with MetS or rheumatoid arthritis [40, 91, 95-99]. Furthermore, we recently showed, in a prospective analysis, that over a 7-year period C3 was independently associated with longitudinal changes in HOMA [100]. Also, C3 was associated with fasting insulin, and in Pima Indians, an ethnic group with an increased propensity for obesity and increased cardiometabolic risk, the association of C3 with fasting insulin was independent of body fat percentage $[42,91,92,94,101]$. In the Pima Indians, C3 also correlated with postprandial insulin levels after a glucose challenge, and was, independently of confounding factors, inversely associated with clamp insulin sensitivity. In an analysis of the circulating proteome, C3 also emerged as one of the strongest determinants of fasting insulin [39]. The observation that $\mathrm{C} 3$ expression in adipose tissue was not affected by external insulin infusion [50] supports the concept that C3 might influence insulin concentrations, and not vice versa. The accumulating evidence for a role of $\mathrm{C} 3$ in insulin resistance raises the question of which specific complement activation pathways may be involved herein, and several studies have addressed the role of specific complement components and activation products in human insulin resistance. C3a was correlated with fasting insulin in Caucasian and Chinese individuals, but not in the Pima Indians [42, 92, 102], and was also independently of age and sex associated with HOMA $[100,102]$. An independent association of sC5b-9 with HOMA was also reported [103]. Higher plasma concentrations of both FH and FB were associated with insulin resistance, and $\mathrm{FH}$ but not $\mathrm{FB}$ decreased with improved insulin resistance induced by rosiglitazone treatment [45]. Furthermore, C3, properdin, FB and FH all showed age-and sexadjusted correlations with HOMA in South Asian non-diabetic subjects [87]. Data on FD have been inconsistent. FD correlated with HOMA in a cohort of obese and non-obese adults and correlated with insulin in overweight to obese women [29, 32]. However, FD was not elevated in insulin-resistant compared to insulin-sensitive morbidly obese young adults [104]. In a small study, only C3, but not C3a, C1q, FB, Bb or FD was inversely associated with 
clamp insulin sensitivity, although there was a trend for C1q and FB [105]. The lectin pathway, on the other hand, might have a beneficial role in insulin resistance, as low MBL levels were observed to be associated with reduced insulin sensitivity during clamp, or no association with HOMA was found $[66,106,107]$. In these studies MBL was inversely correlated with inflammation, and a beneficial role for $\mathrm{MBL}$ was hypothesized to emerge from a potential protection against chronic infections.

Overall, a growing body of evidence suggests a role of C3 in systemic insulin resistance, which may primarily involve terminal and alternative pathway complement activation. The lectin pathway may actually exert protective effects in insulin resistance while there is little data with respect to the classical pathway.

\section{The complement system in the metabolic syndrome and type 2 diabetes}

The association of complement with systemic insulin resistance and inflammation suggests that complement might also contribute to DM2 and MetS, which is a strong risk factor for DM2. Complement C3 is elevated in MetS in persons of different ethnicities and is correlated with the number of overt MetS components [94, 108-111]. The association between C3 and prevalent MetS was shown to be independent of plasma cholesterol, insulin resistance and inflammation $[97,110]$. Notably, the association of C3 with incident MetS was also shown to be independent of baseline central obesity, lifestyle factors and use of medication [83, 94]. This suggests that specific pathways, additional to general inflammatory mechanisms, may underlie the association of C3 with MetS. Potentially, a role of C3 and/or C3 activation in postprandial lipid metabolism might provide the missing link between C3 and MetS risk. In a large study, the strength of the association of C3 with risk of developing the MetS was doubled in individuals who consumed a high amount of dietary fat [94]. A blunted increase in C3 and/or C3a-desArg/ASP after fat consumption might impair peripheral lipid metabolism $[97,112]$, although other, smaller studies on postprandial increases in C3 or C3a-desArg/ASP were not conclusive [50, 113-116]. A polymorphism in C3 that was protective in the development of MetS was noted to be associated with higher plasma polyunsaturated fatty acids and lower triacylglycerides [111], which further supports a function for C3 in postprandial FFA and triacylglyceride metabolism.

The role of $\mathrm{C} 3$ and its activation products in DM2 has been investigated in several crosssectional and prospective studies. In cross-sectional studies, C3 was found to be elevated in patients with DM2 of European and Asian ethnicity [117-119]. The association of C3 with incident DM2 has been investigated in 4 large studies ( $n \sim 500$ to 13,000): in middle-aged men, C3 was independently associated with incident DM2 over a 6-year period [80]. In middleaged to elderly individuals, a borderline significant association of C3 with incident DM2 was apparent only in women, but not in men [83]. This study, however, had a follow-up time of only 3.3 years and may therefore have been underpowered, as suggested by the low number of incident DM2 cases $(n=30)$. In middle-aged US-American subjects with different ethnicities, C3 was independently associated with incident DM2 during 7.6 years of followup [120]. Furthermore, in individuals with a moderately increased cardiometabolic risk we showed that the association of C3 with 7-year incidence of DM2 was independent of low- 
grade inflammation [100]. Further evidence indicates that C3 activation might be involved in the development of DM2: the activated fragment C3d was elevated in patients with DM2 [121] and C3a was correlated with 7-year DM2 incidence, but this association was not independent of confounding factors [100]. In middle-aged women, the activated fragment C3c was independently associated with 16-year incidence of DM2 [122], although it is not completely clear if the assay used was specific for C3c or also captured non-activated C3. Interestingly, this study investigated the association of environmental air pollution with DM2, and observed an interaction between $\mathrm{C} 3 \mathrm{C}$ and air pollution on DM2 risk. This suggests that metabolic as well as environmental factors that stimulate chronic inflammatory pathways may contribute to C3 activation and, thereby, to development of DM2.

Other complement factors have only occasionally been studied in relation to DM2. sC5b-9 was not elevated in patients with DM2 and was not associated with incident DM2 [100, 117, 119]. In support of the relevance of the terminal pathway, $C 5$ and $C 8$ were elevated in Swedish DM2 patients [123]. Furthermore, C4, C4d, FB, Bb, iC3b and C3a were elevated in Japanese patients with DM2 [117]. In addition, the association of complement with family history of DM2 was investigated in South Asian and Caucasian individuals. Properdin, FB and sC5b-9 were independently of insulin resistance and other metabolic traits associated with family history of DM2 [87]. C3 and FB, but not C1q, C4 or FD, were borderline significantly elevated in first-degree relatives of DM2 patients [84]. These findings support a potential role of the alternative pathway in the predisposition for DM2. In overt DM2, complement was also linked to hyperglycaemia. Reduction in $\mathrm{HbA} 1 \mathrm{c}$ achieved with anti-hyperglycaemic therapy was accompanied by decreases in C3 [124, 125], while C5 and C8 correlated with HbA1c [123], and MASP-2, but not sC5b-9, correlated weakly with HbA1c [68].

Altogether, a substantial body of data suggests an association of C3 with MetS, potentially involving a role of C3 in postprandial lipid metabolism. Several large studies have shown C3 to be a predictor of DM2, independent of confounding factors such as (central) obesity or low-grade inflammation. Some small studies suggest involvement of the terminal and the alternative pathway, and limited evidence suggests complement to be linked to hyperglycaemia. Further studies are needed to elucidate the biological pathways that are involved.

\section{The complement system in fatty liver disease}

The liver plays a pivotal role in the metabolic impairments that result from obesity-induced inflammation, insulin resistance and dyslipidaemia. Non-alcoholic fatty liver disease (NAFLD) is regarded to be the liver manifestation of the MetS and DM2, and up to $80 \%$ of obese individuals have been reported to have some form of NAFLD [126]. In fact, hepatic fat accumulation rather than (central) adiposity itself has been proposed as the cause of systemic insulin resistance [127].

Fat accumulation in hepatocytes induces fatty liver (steatosis), and results in metabolically compromised and insulin-resistant hepatocytes. Excess intracellular FFAs and lipid peroxidation products are toxic, produce mitochondrial defects and initiate an inflammatory response. In NAFLD, steatosis-induced cell injury and inflammation (steatohepatitis) is 
primarily enforced by obesity-induced inflammatory mediators. In the alcohol-driven form of fatty liver disease, alcoholic liver disease (ALD), alcohol metabolites stimulate fat synthesis and decrease fat egress, while the inflammatory response is aggravated by intestinal products such as translocated lipopolysaccharide. Fat-laden, inflamed hepatocytes are likely to die, and apoptotic and necrotic hepatocytes together with the inflammatory environment stimulate hepatic Kupffer and stellate cells. Activated Kupffer cells push inflammation and further stimulate stellate cells to induce tissue fibrosis, which ultimately can result in liver cirrhosis $[128,129]$. Thus, NAFLD can to a certain extent result in similar liver damage as observed in ALD, but these two conditions differ with respect to the underlying trigger for hepatic fat accumulation.

The liver has long been recognized as the major organ for the production of complement factors, but complement may also play an active role in liver disease [130, 131]. In humans, complement has been associated with indirect as well as with direct measures of liver damage. First, circulating C3 was associated with prevalent hepatic steatosis assessed by ultrasound [96]. Secondly, circulating C3 has, independently of other metabolic parameters, been associated with markers of liver dysfunction [132, 133]. Thirdly, studies that had human liver biopsy material available strongly suggest that complement is altered in liver damage. In livers of patients with NAFLD, increased deposition of C1q, MBL, C4d, C3c and C5b-9 was evident, while C4d, C3c and C5b-9 deposits were absent in healthy livers [134]. In this study, the degree of C5b-9 deposition correlated with disease severity. Furthermore, increased C3 mRNA expression was found in liver samples of patients with severe NAFLD [135]. Interestingly, C3 and C3a/ASP were elevated in patients with NAFLD, not only compared to controls but also compared to patients with chronic viral hepatitis, which underscores a role of C3 and C3a/ASP in NAFLD beyond generalized inflammation [102]. MBL was detected to a higher degree in fatty livers, but was also strongly present in healthy livers. Also $\mathrm{MBL}$ genotypes, which are commonly used as proxy of MBL plasma concentrations, were not associated with presence of NAFLD [134]. The only study on the alternative pathway did not observe FD to be elevated in patients with biopsy proven NAFLD [136]. These observations suggest the classical and the terminal pathway to be affected in liver disease, while the role of the lectin pathway remains unclear.

These human studies are complemented by in vitro and animal studies that explore the function of complement in NAFLD. The anaphylatoxin receptors C3aR and C5aR1 are expressed on resting Kupffer and stellate cells, and expression can be induced by proinflammatory stimuli [131]. C3a and C5a were shown to activate Kupffer and stellate cells in vitro [137, 138], and inhibiting C5a signalling had anti-fibrotic effects [139]. This suggests that complement activation occurs in the course of tissue fibrosis after hepatocellular injury. High-fat feeding-induced steatosis showed a role for C1q and C3 activation in steatosis in transgenic $\mathrm{C}_{1 q^{--}}, \mathrm{C}_{3} \mathrm{aR}^{-/-}$or $\mathrm{CD} 55(\mathrm{DAF})^{-/-}$mice $[56,58]$. Interestingly, $\mathrm{C}^{-/-}$mice on a high-fat diet developed more steatosis than control mice, which suggests that a minimum levels of functional C3 may actually be beneficial in high-fat feeding [140]. Furthermore, serum C2 and C8 were altered in swine with diet-induced liver steatosis and inflammation [141]. These 
studies in diet-induced steatosis again implicate classical, C3 and terminal activation in NAFLD.

Studies in ALD illustrate specific mechanisms of ALD as well as general pathobiological mechanisms of liver damage that may be comparable to NAFLD. In humans, alcohol consumption was observed to alter the effects of complement on markers of liver dysfunction, as C3a was observed to be independently associated with a composite liver enzyme score in heavy drinkers, but not in mild-to moderate drinkers [142]. In support of these human data, several animal studies also showed C3 activation to contribute to alcoholinduced liver damage. Mice deficient in $\mathrm{C} 3\left(\mathrm{C}^{--}\right)$were protected against ethanol-induced steatosis, while mice deficient in CD55 (DAF) developed more steatosis after alcohol feeding $[140,143]$. Of note, alcohol and alcohol metabolites may directly activate C3, as supported by increased C3a levels and increased hepatic C3b deposition in alcohol-fed mice [143, 144]. $\mathrm{C}^{\%}$ mice were not protected against ethanol-induced steatosis, but hepatic $\mathrm{C} 8$ deposition in alcohol-fed rats implicates activation of the terminal pathway [145]. C1 $\mathrm{q}^{-/-}$mice were protected against alcohol-induced steatosis, and $\mathrm{C} 1 \mathrm{q}$ activation on hepatocytes promoted C3b deposition and apoptosis [146].

In summary, complement seems to play a role in both NAFLD and ALD, but human data are limited. Animal studies suggest that alcohol may activate C3, while functional C3 appeared to be beneficial in high-fat-induced fatty liver. Although there is sufficient evidence to support a role of $\mathrm{C} 3$ and its immediate activation products in NAFLD, little is known about other complement factors. Indices of classical and terminal pathway activation were found in human fatty livers, while there are no comprehensive studies on the alternative or the lectin pathway.

\section{Complement in cardiovascular disease}

Cardiovascular disease (CVD) is a major clinical manifestation of cardiometabolic disorders and is characterized by critically narrowing (stenosis) or occlusion (atherothrombosis) of blood vessels. Coronary heart disease comprises partial or complete obstruction of coronary vessels, while the term CVD additionally includes partial or complete obstruction of peripheral or cerebral vessels. Key processes in CVD are endothelial dysfunction, atherosclerosis, and impaired regulation of coagulation and fibrinolysis. The complement system may be involved in all these processes based on its immune, inflammatory and metabolic functions.

\subsection{Complement in initiation of CVD}

The endothelium regulates crucial functions of blood vessels such as vascular tone, vascular permeability and coagulation, and may also be a source of complement factors such as properdin [147]. In endothelial dysfunction, the capacity for vasodilation is reduced, which impairs blood pressure regulation. Furthermore, dysfunctional endothelial cells express adhesion molecules that promote adherence and migration of inflammatory cells, which initiates and furthers atherosclerosis. The complement system is thought to contribute to several aspects of endothelial dysfunction $[148,149]$. Endothelial cells express anaphylatoxin receptors and complement regulators on their surface, which indicates that they are a direct 
target of complement. In vitro, C3a, C5a and C5b-9 induced expression of adhesion molecules and proinflammatory cytokines in endothelial cells, and (s)C5b-9 was shown to be involved in leakage of the endothelial cell layer [150]. We recently showed that, in humans, C5a and sC5b-9 were independently associated with plasma markers of endothelial dysfunction [88]. Also in another study, sC5-9 was independently associated with soluble Eselectin [103].

When the endothelial lining of blood vessels is compromised, entrance of lipids and inflammatory cells into the sub-endothelial space initiate and further atherosclerosis. Complement may have a dual function in atherosclerosis, as it is involved in the removal of debris but also in the amplification of the inflammatory response and the recruitment of immune cells [151-153]. Complement is activated in early fatty streaks and also in late stages of atherosclerosis (major plaque/atheroma). In the plaque, complement co-localized with potential activators such as CRP, oxidized low-density lipoproteins (LDL), enzymatically modified LDL, cholesterol crystals, gC1qR and macrophages/foam cells. Furthermore, mRNA for C1q, C1r, C1s, C4, C7 and C8 was detected in human plaques, which suggests local production of complement $[151,154]$. Genetically modified animal models (reviewed in [155]) suggest that $\mathrm{C} 3 \mathrm{a}, \mathrm{C} 5 \mathrm{a}$ and $\mathrm{C} 5 \mathrm{~b}-9$ may drive atherosclerosis. In contrast, the pattern recognition molecules $\mathrm{C} 1 \mathrm{q}$ and $\mathrm{MBL}$ appeared to have protective effects, which may be explained by a role in the removal of cellular debris. A possible protective role of C3 in mice was attributed to a role of C3 in lipid metabolism (see [155]).

\subsection{Human studies on complement in CVD}

A relatively large body of data has been published on the association of C3 with human CVD. C3 was consistently shown to be elevated in patients with prevalent CHD, independently of obesity [156-158], or inflammatory markers [101, 159]. We reported similar associations, but additionally showed that this association may be largely attributable to the subset of heavy smokers [160]. The associations of C3 with incident CVD are somewhat less consistent (table 2). In two medium-sized populations ( $\mathrm{n} 1000$ ), associations between C3 and risk of CHD were observed when adjusted for age and sex [161], or additionally for smoking, MetS and CRP [83]. On the other hand, in a large study ( $n>5000$ men), this association was not independent of obesity, lipids, and diabetes [162]. Interestingly, in that same study, C3 did emerge as an independent predictor of incidence of hypertension [163], while studies on C3 and incident stroke were inconsistent $[162,164]$. C3 was also associated with incidence of atrial fibrillation, but not independently of other risk factors [165]. Interestingly, in several large, observational studies, C3 was not independently associated with severity of atherosclerosis, i.e. intima-media thickness (IMT) $[89,166,167]$. Thus, it appears that C3 may play distinct roles in different aetiological processes of CVD, and may potentially contribute to CVD via mechanisms other than atherosclerosis. Moreover, other cardiometabolic risk factors may confound or modify the effect of $\mathrm{C} 3$ on risk of CHD.

Downstream activation products of $\mathrm{C} 3$ show partly dissimilar associations. We recently reported that in contrast to $\mathrm{C} 3$, C3a was independently associated with IMT and anklebrachial index (ABI), another marker of atherosclerosis [89]. We also observed a strong and independent association of $\mathrm{C} 3 \mathrm{a}$ with prevalent CVD in heavy smokers, which was 
independent of C3 and was not explained by low-grade inflammation. This suggests that C3 and C3a may reflect a different aspect of the association of the complement system with CVD. In addition, previous case-control studies have shown C3a to be elevated in CHD patients [168], in patients with angina pectoris [169], and the fragment C3d to be elevated in CHD patients with T2DM [121]. In a proteomic study, C5a was identified as more abundant in plasma of CHD patients compared to controls [170]. In contrast, we did not observe an association of $\mathrm{C} 5 \mathrm{a}$ and $\mathrm{sC} 5 \mathrm{~b}-9$ with prevalent CVD or with markers of atherosclerosis IMT or $A B I$ [88]. Others reported sC5b-9 to be elevated in patients with peripheral arterial disease and heart failure $[86,103,171]$, while studies on sC5b-9 in angina pectoris were discrepant [172-174]. Two studies that investigated sC5b-9 in CHD patients with different degrees of vessel obstruction (stenosis) also yielded conflicting findings [174, 175].

Table 2: Longitudinal studies ( $\mathrm{N}>150$ ) on complement factors and risk of a first cardiovascular event

\begin{tabular}{|c|c|c|c|c|c|c|c|}
\hline \multirow[t]{2}{*}{ Publication } & \multicolumn{2}{|l|}{ Population } & \multirow[t]{2}{*}{ Outcome } & \multirow[t]{2}{*}{ Follow-up } & \multicolumn{3}{|c|}{ C-factor Association with outcome } \\
\hline & Participants & $\mathrm{N}$ at risk / cases & & & & Crude $^{a}$ & Multivariable ${ }^{b}$ \\
\hline Muscari 1995 [161] & men \& women & $860 / 57$ & $\mathrm{CHD}$ & 4 yrs & C3 & positive & n.r \\
\hline Onat 2010 [83] & men \& women & $1145 / 84$ & CHD & 3.3 yrs & C3 & n.r. & positive \\
\hline $\begin{array}{l}\text { Adamsson } 2011 \\
\text { [165] }\end{array}$ & men & $6031 / 667$ & $\begin{array}{l}\text { atrial } \\
\text { fibrillation }\end{array}$ & 25 yrs & $\begin{array}{l}\mathrm{C} 3 \\
\mathrm{C} 4\end{array}$ & $\begin{array}{l}\text { positive } \\
\text { none }\end{array}$ & $\begin{array}{l}\text { positive } \\
\text { none }\end{array}$ \\
\hline $\begin{array}{l}\text { Cavusoglu } 2007 \\
{[164]}\end{array}$ & men & $389 / 23$ & stroke & 2 yrs & $\begin{array}{l}\mathrm{C} 3 \\
\mathrm{C} 4\end{array}$ & $\begin{array}{l}\text { positive } \\
\text { positive }\end{array}$ & $\begin{array}{l}\text { positive } \\
\text { positive }\end{array}$ \\
\hline $\begin{array}{l}\text { Engstrom } 2007 \\
\text { [162] }\end{array}$ & men & $5850 / 185$ & CVD & 18 yrs & $\begin{array}{l}\mathrm{C} 3 \\
\mathrm{C} 4\end{array}$ & $\begin{array}{l}\text { positive } \\
\text { positive }\end{array}$ & $\begin{array}{l}\text { none } \\
\text { positive i }\end{array}$ \\
\hline Luc 2010 [82] & men & $9779 / 617^{c}$ & $\mathrm{CHD}$ & 10 yrs & FD & none & none \\
\hline Prugger 2012 [189] & men & $9771 / 95 d$ & stroke & 10 yrs & FD & positive & positive \\
\hline Best 2004 [179] & men \& women & $4549 / 217^{\mathrm{e}}$ & $\mathrm{CHD}$ & $7-10$ yrs & $M B L 2$ & inverse & inverse \\
\hline $\begin{array}{l}\text { Siezenga } 2011 \\
\text { [181] }\end{array}$ & $\begin{array}{l}\text { men \& women } \\
\text { with T2DM }\end{array}$ & $168 / 31$ & CVD & 7.6 yrs & $\begin{array}{l}\text { MBL2 } \\
\text { MBL }\end{array}$ & $\begin{array}{l}n . r \\
n . r\end{array}$ & $\begin{array}{l}\text { inverse } \\
\text { none }\end{array}$ \\
\hline Vengen 2012 [182] & men \& women & $57133 / 370^{f}$ & $\mathrm{CHD}$ & $11-13$ yrs & $M B L 2$ & inverse & inverse \\
\hline Keller 2006 [81] & men \& women & $25663 / 946^{\mathrm{g}}$ & $\mathrm{CHD}$ & $6 \mathrm{yrs}$ & MBL & $n . r$ & positive ${ }^{J}$ \\
\hline $\begin{array}{l}\text { Saevarsdottir } 2005 \\
\text { [180] }\end{array}$ & men \& women & 19381 / 867 h & $\mathrm{CHD}$ & 27 yrs & $\mathrm{MBL}$ & none & none \\
\hline \multicolumn{8}{|c|}{ MBL2, MBL-genotype; MBL, systemic MBL concentrations, n.r. not reported, yrs, years } \\
\hline 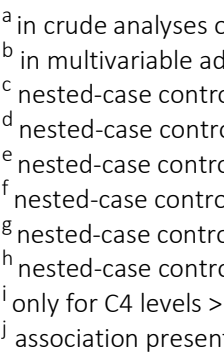 & $\begin{array}{l}\text { or in age- and sex } \\
\text { djusted analyses; } \\
\text { rol study, } 1215 \text { ma } \\
\text { rol study, } 190 \text { mat } \\
\text { ol study, } 217 \text { mat } \\
\text { ol study, } 370 \text { mat } \\
\text { ol study, } 1799 \text { ma } \\
\text { ol study, } 442 \text { mat } \\
\text { 90th percentile; } \\
\text { ht only in men }\end{array}$ & $\begin{array}{l}\text {-adjusted analyse } \\
\text { ched controls; } \\
\text { ched controls; } \\
\text { ched controls; } \\
\text { ched controls; }\end{array}$ & & & & & \\
\hline
\end{tabular}


In addition to C3 and its downstream activation products, an array of other complement components has also been evaluated in relation to human CVD. The complex C1sC1rC1-INH is elevated in patients with CHD, while C1q was not different between T2DM patients with or without CHD $[174,176]$. C4 and C4d do not appear to be altered in angina pectoris or in heart failure $[172,177]$, and C4 was associated with risk of stroke in a small but not in a large study $[162,164]$. The latter study did report an independent association of very high C4 levels (above the $90^{\text {th }}$ percentile) with CHD. C1-INH did not differ between patients with different clinical presentations of angina pectoris [169], although it was associated with arterial stiffness, independently of other cardiovascular risk factors [85]. C4BP was elevated in patients with peripheral arterial disease compared to controls [86], and was associated with severity of atherosclerosis [178]. C4BP was also detected in human atherosclerotic aortas [178]. Since C1-INH, C4 and C4BP are shared by the classical and the lectin pathway, these studies suggest that dysfunctional regulation of classical and lectin pathway activation may contribute to arterial stiffening and atherosclerosis. In contrast, a potential antiatherosclerotic effect has been suggested for the initiation molecule of the lectin pathway. Lower MBL plasma concentrations or low MBL genotypes were associated with an increased risk of CHD in several larger longitudinal studies [179-182]. Only one study found high MBL levels to be associated with CHD, and this association was only found in men but not in women [81]. An independent association of plasma MBL with IMT was observed in patients with rheumatoid arthritis [183]. Interestingly, this latter study reported a U-shaped relation with IMT, denoting that both low and high MBL levels may be unfavourable in the development of atherosclerosis. Such a complex role of MBL in atherosclerosis may explain why some studies assessing MBL2 genotype did not find a clear effect of $M B L 2$ on atherosclerosis: $M B L 2$ genotype was in young and healthy men not associated with early atherosclerotic changes [184] and was in obese individuals not associated with IMT or ABI [185]. In patients with CAD, MBL2 was not associated with baseline or 2-year progression of coronary atherosclerosis [186]. With respect to the alternative pathway, polymorphisms in $\mathrm{FH}$ have long been discussed as risk factor for $\mathrm{CHD}$, but two large meta-analyses concluded that the commonly studied $\mathrm{Y} 402 \mathrm{H}$ variant ( $\mathrm{rs} 1061170$ ) is not associated with $\mathrm{CHD}[187,188]$. Interestingly, in two large studies, FD was not associated with risk of CHD, but was independently associated with risk of stroke $[82,189]$. Furthermore, in a proteomic study FB was detected as an elevated plasma component in CHD patients [170]. On the other hand, alternative pathway activation markers $\mathrm{C} 3 \mathrm{bBbP}$ and $\mathrm{Bb}$ were not elevated in patients with angina, or in patients with stenosis of coronary arteries [172, 174]. Taken together, the picture emerges that the classical, the lectin and the alternative pathway may each play specific roles in the development of human CVD.

In addition to early and chronic CVD, complement also appears to contribute to advanced cardiovascular morbidity. In patients with established CVD, complement activation has been associated with risk of progression of CVD and with secondary events. Activated complement components were detected in human infarcted myocardium and C3, C3a, C5a and C1q were detected in coronary thrombi [190]. C5a and sC5b-9 were repeatedly shown to increase during acute CVD events, where they are thought to mediate ischaemia-reperfusion injury 
$[191,192]$. Other complement activation products, i.e. Bb, C4d, C3d and MASP-1 were also increased in patients with acute myocardial infarction [69, 172, 193]. In several studies, sC5b-9 levels at admission for acute myocardial infarction predicted risk of death or secondary events $[68,194,195]$. In hospitalized heart failure patients, sC5b-9 correlated with functional outcome and survival [171]. Also C5a was, in patients with CHD or peripheral arterial disease, associated with secondary CVD events or restenosis [196-198]. This suggests that the degree of terminal complement activation during acute events or in severe CVD may reflect severity of the event. $\mathrm{C} 3$ was also shown to contribute to progression of disease in established CVD. In patients with CHD, C3 was independently associated with the risk of secondary events and with restenosis $[158,199]$. Moreover, the C3/C4 ratio was, in CHD patients, independently associated with risk of secondary events [200]. In contrast, in stroke patients, C3 and C4 were not associated with survival or secondary events [201, 202]. Consistently with the above-described apparent anti-atherosclerotic effect of $\mathrm{MBL}$, low $\mathrm{MBL}$ was associated with higher risk of secondary events $[67,181]$. In contrast, low MBL was associated with a lower risk of restenosis [199]. In patients with high MBL, low levels of C1INH suggesting less inhibition of the lectin pathway, were also associated with restenosis [203]. MASP-2 on the other hand was not observed to be associated with secondary events in patients with acute $\mathrm{MI}$ [68]. These data suggest a complex relation between the lectin pathway and the processes that underlie cardiovascular events on the one, and restenosis on the other hand.

Overall, C3 has been investigated in several large studies, and it appears that that the association of C3 with risk of CVD may be modulated by other CVD risk factors. C3 may affect distinct aetiological processes in CVD, while C3a appears to reflect the severity of atherosclerosis. Systemic activation of the terminal pathway seems to be prominent in acute CVD events and in advanced stages of CVD. In contrast, the lectin pathway appears to have a dual role in CVD, as several studies point to a beneficial function of the lectin pathway in anti-atherosclerotic mechanisms, while some studies showed adverse effects of high MBL levels. The role of the classical and the alternative pathway has so far only been marginally studied.

\subsection{Novel insights in complement-related CVD mechanisms: HDL, diabetic complications, fibrinolysis}

Ongoing research has revealed further emerging links between distinct aspects of cardiometabolic disease and the complement system that may contribute to increased CVD risk, of which we provide a few examples. First, high-density lipoprotein (HDL) has in humans been recognized as carrier of complement, as shown for C1q, C1s, C2, C3, C4, C5, C9, FB, FH, C1-INH and vitronectin [204-206]. Interestingly, C3 was enriched in HDL from patients with CHD compared to HDL from controls [206]. Furthermore, plasma C3 correlated with presence of pro-atherogenic HDL particles in patients with systemic lupus erythematosus [207]. These observations suggest that complement may influence the (anti-)inflammatory properties of $\mathrm{HDL}$ in cardiometabolic diseases.

Secondly, interaction between complement and cardiometabolic disease may be related to (transient) hyperglycaemia. Glycation of CD59 correlated with glucose control in DM2 and 
was shown to interfere with its protective function [208, 209]. For example, in kidneys from patients with DM2, glycated CD59 co-localized with C5b-9, while deposition of C5b-9 was absent in healthy kidneys [210]. These findings suggest that in persons with impaired glucose metabolism or DM2, as often seen in cardiometabolic diseases, glycation of complement regulator(s) - particularly CD59 - may increase the susceptibility for vascular damage.

Thirdly, complement might also to contribute to CVD via its functions in coagulation and fibrinolysis [21, 211]. In humans, plasma C3 was found to be associated with fibrinogen, platelet count or plasminogen activator inhibitor-1 (PAI-1) [156, 157, 212, 213]. Moreover, C3 is now recognized to be a structural component of blood clots. In healthy volunteers, C3 was shown to bind to fibrinogen, and plasma C3 concentrations correlated with the extent of C3 incorporation into fibrin clots [214]. Within the clot, the amount of C3 incorporation was observed to affect clot structure and sensibility to lysis. In line with this, plasma C3 was shown to be associated with clot lysis time, independently of PAI-1 and fibrinogen [215]. Furthermore, in patients with diabetes the effect of $\mathrm{C} 3$ and clot parameters may be affected by glycaemic control [216]. These observations suggest that complement may have specific effects in fibrinolysis that may partly explain distinct associations of different aspects of complement activation with atherosclerotic and atherothrombotic manifestations of CVD.

\section{Lifestyle factors and drugs that influence complement}

Given the multi-facetted implications of complement in cardiometabolic disease, it appears valuable to consider the effect of modifiable lifestyle factors and commonly prescribed drugs on complement. Circulating C3 was in observational studies associated with dietary intake of provitamin A, selenium status and dietary anti-oxidant intake [217-219]. An experimental study that compared the effects of two hypocaloric diets on C3 found that a legume-based diet produced a stronger decrease of plasma C3 than an isocaloric diet that was comparable in macronutrient pattern. Importantly, this association was persistent after adjustment for the extent of weight loss. Besides, there are some indications that physical activity and fitness are related to C3 [220, 221]. Furthermore, some commonly prescribed anti-hypertensive, lipid-lowering and glucose-lowering drugs were shown to have an effect on C3, C3a or C4BP $[124,125,130,222-225]$. Together, these preliminary observations suggest that C3 levels can be affected by micronutrient intake, diet quality and physical activity independently form the control of body weight, and indicate a potential to influence C3 levels via widely used drugs.

\section{Conclusion}

The above-presented data suggest that the complement system may provide a link between adipose tissue inflammation and systemic metabolic derangements that promote human cardiometabolic disease (figure 1). Complement appears to play a role in general but also in specific processes of cardiometabolic disease, such as lipid metabolism, hyperglycaemia and fibrinolysis. A large array of studies has investigated C3, and shown independent associations with insulin resistance, liver dysfunction and in the risk of MetS and T2DM. C3 was also associated with CVD; however the risk of C3 with CVD may be modulated by other CVD risk 
factors such as smoking or dietary fat. The role of the three activation pathways as well as downstream activation of C3 is needs to be studied in more detail, but available evidence suggests that they have distinct roles in processes underlying cardiometabolic disease.

For the future, large, longitudinal studies are awaited that can investigate which aspects of complement activation or regulation affect the different processes in human cardiometabolic disease. In particular, studies that measure several complement factors within one and the same cohort are needed, and studies that allow sub-analyses in certain risk groups to investigate interactions of complement with relevant other cardiovascular risk factors. Furthermore, novel insights will be extracted from investigations on how complement is influenced by genotype (complotype), lifestyle and (pre-existing) pharmacological treatments. 

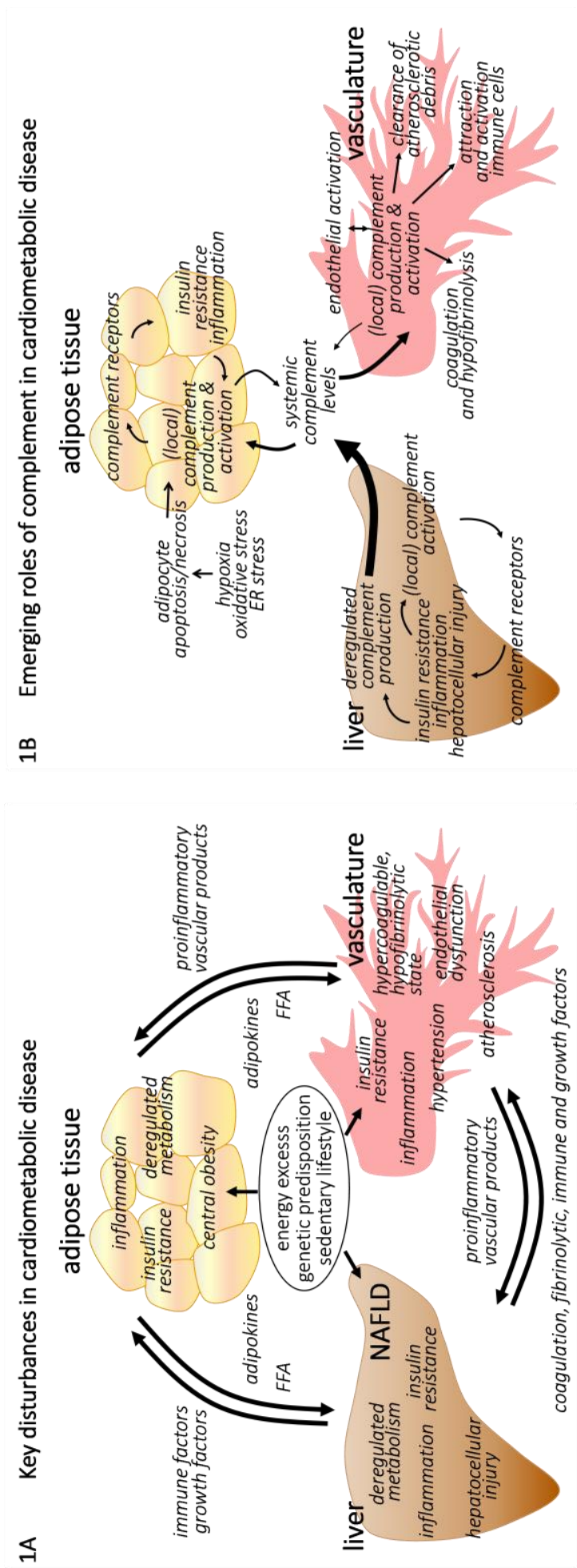

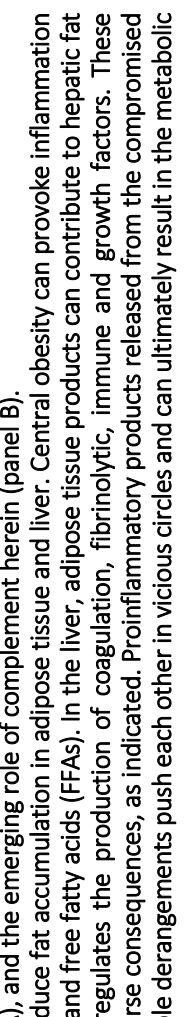

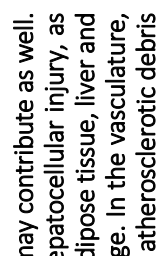

है

뜽뭉응 웡

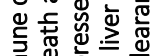

등

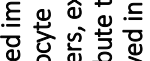

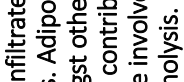

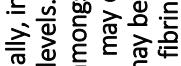

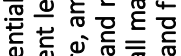

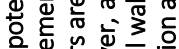

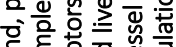

के है 웡웡

넌

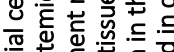

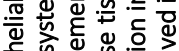

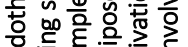

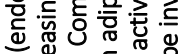

논

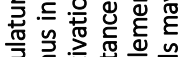

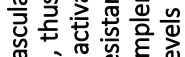

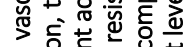

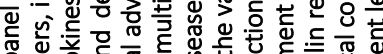

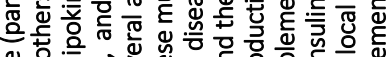

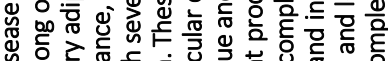

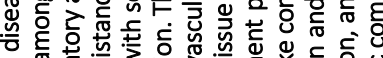

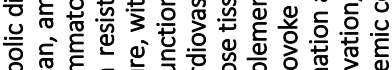

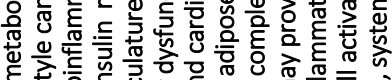

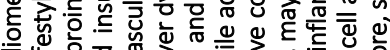

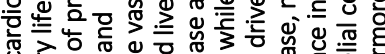

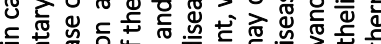

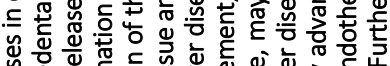

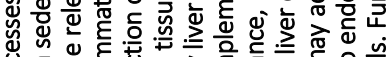

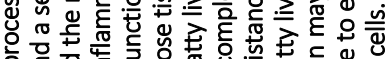

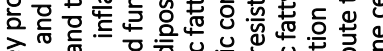

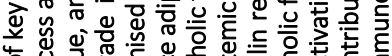

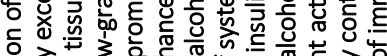

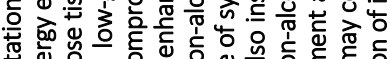

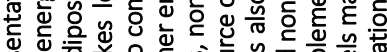

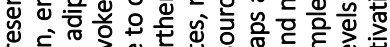

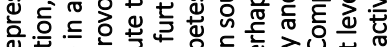

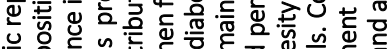

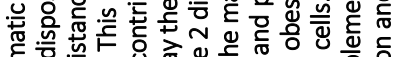

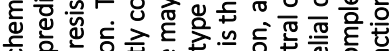
员. 든

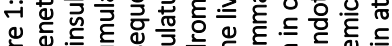

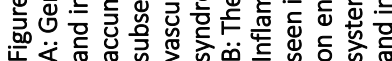




\section{References}

1. Gill H, Mugo M, Whaley-Connell A, Stump C, Sowers JR. The key role of insulin resistance in the cardiometabolic syndrome. Am. J. Med. Sci., 2005. 330: 290-4.

2. Castro JP, El-Atat FA, McFarlane SI, Aneja A, Sowers JR. Cardiometabolic syndrome: pathophysiology and treatment. Curr. Hypertens. Rep., 2003. 5: 393-401.

3. Kaur J. A Comprehensive Review on Metabolic Syndrome. Cardiol. Res. Pract., 2014. 2014: 943162.

4. Trayhurn P. Hypoxia and adipose tissue function and dysfunction in obesity. Physiol. Rev., 2013. 93: 1-21.

5. Dali-Youcef N, Mecili M, Ricci R, Andres E. Metabolic inflammation: connecting obesity and insulin resistance. Ann. Med., 2013. 45: 242-53.

6. van Greevenbroek MM, Schalkwijk CG, Stehouwer CD. Obesity-associated low-grade inflammation in type 2 diabetes mellitus: causes and consequences. Neth. J. Med., 2013. 71: 174-87.

7. Capurso C, Capurso A. From excess adiposity to insulin resistance: the role of free fatty acids. Vascul. Pharmacol., 2012. 57: 91-7.

8. Item F, Konrad D. Visceral fat and metabolic inflammation: the portal theory revisited. Obes. Rev., 2012. 13 Suppl 2: 30-9.

9. Fabbrini E, Sullivan S, Klein S. Obesity and nonalcoholic fatty liver disease: biochemical, metabolic, and clinical implications. Hepatology, 2010. 51: 679-89.

10. Yki-Jarvinen H. Liver fat in the pathogenesis of insulin resistance and type 2 diabetes. Dig. Dis., 2010. 28: 203-9.

11. Turner N, Cooney GJ, Kraegen EW, Bruce CR. Fatty acid metabolism, energy expenditure and insulin resistance in muscle. J. Endocrinol., 2014. 220: T61-79.

12. Kahn SE, Cooper ME, Del Prato S. Pathophysiology and treatment of type 2 diabetes: perspectives on the past, present, and future. Lancet, 2014. 383: 1068-83.

13. Karaca U, Schram MT, Houben AJ, Muris DM, Stehouwer CD. Microvascular dysfunction as a link between obesity, insulin resistance and hypertension. Diabetes Res. Clin. Pract., 2014. 103: 382-387.

14. Libby P. Inflammation in atherosclerosis. Arterioscler. Thromb. Vasc. Biol., 2012. 32: 2045-51.

15. Faber DR, de Groot PG, Visseren FL. Role of adipose tissue in haemostasis, coagulation and fibrinolysis. Obes. Rev., 2009. 10: 554-63.

16. Phillips CM. Metabolically healthy obesity: definitions, determinants and clinical implications. Rev. Endocr. Metab. Disord., 2013. 14: 219-27.

17. Ricklin D, Hajishengallis G, Yang K, Lambris JD. Complement: a key system for immune surveillance and homeostasis. Nat. Immunol., 2010. 11: 785-97.

18. Noris M, Remuzzi G. Overview of complement activation and regulation. Semin. Nephrol., 2013. 33: 479-92.

19. Degn SE, Thiel S. Humoral pattern recognition and the complement system. Scand. J. Immunol., 2013. 78: 181-93.

20. Harboe M, Mollnes TE. The alternative complement pathway revisited. J. Cell. Mol. Med., 2008. 12: $1074-84$.

21. Oikonomopoulou K, Ricklin D, Ward PA, Lambris JD. Interactions between coagulation and complement--their role in inflammation. Semin. Immunopathol., 2012. 34: 151-65.

22. Klos A, Wende E, Wareham KJ, Monk PN. International Union of Basic and Clinical Pharmacology. [corrected]. LXXXVII. Complement peptide C5a, C4a, and C3a receptors. Pharmacol. Rev., 2013. 65: 500-43.

23. Nesargikar PN, Spiller B, Chavez R. The complement system: history, pathways, cascade and inhibitors. Eur. J. Microbiol. Immunol., 2012. 2: 103-111.

24. Tegla CA, Cudrici C, Patel S, Trippe R, 3rd, Rus V, Niculescu F, Rus H. Membrane attack by complement: the assembly and biology of terminal complement complexes. Immunol. Res., 2011. 51: 45-60.

25. Bohana-Kashtan O, Ziporen L, Donin N, Kraus S, Fishelson Z. Cell signals transduced by complement. Mol. Immunol., 2004. 41: 583-97.

26. Ricklin D, Lambris JD. Complement in immune and inflammatory disorders: pathophysiological mechanisms. J. Immunol., 2013. 190: 3831-8.

27. Makki K, Froguel P, Wolowczuk I. Adipose Tissue in Obesity-Related Inflammation and Insulin Resistance: Cells, Cytokines, and Chemokines. ISRN Inflamm, 2013. 2013: 139239.

28. Reynolds R, Hartnett ME, Atkinson JP, Giclas PC, Rosner B, Seddon JM. Plasma complement components and activation fragments: associations with age-related macular degeneration genotypes and phenotypes. Invest. Ophthalmol. Vis. Sci., 2009. 50: 5818-27.

29. Derosa G, Fogari E, D'Angelo A, Bianchi L, Bonaventura A, Romano D, Maffioli P. Adipocytokine levels in obese and non-obese subjects: an observational study. Inflammation, 2013. 36: 914-20. 
30. Lehr S, Hartwig S, Lamers D, Famulla S, Muller S, Hanisch FG, Cuvelier C, Ruige J, Eckardt K, Ouwens DM, Sell $\mathrm{H}$, Eckel J. Identification and validation of novel adipokines released from primary human adipocytes. Mol. Cell. Proteomics, 2012. 11: M111 010504.

31. Maslowska M, Vu H, Phelis S, Sniderman AD, Rhode BM, Blank D, Cianflone K. Plasma acylation stimulating protein, adipsin and lipids in non-obese and obese populations. Eur. J. Clin. Invest., 1999. 29: 679-86.

32. Mavri A, Stegnar M, Krebs M, Sentocnik JT, Geiger M, Binder BR. Impact of adipose tissue on plasma plasminogen activator inhibitor-1 in dieting obese women. Arterioscler. Thromb. Vasc. Biol., 1999. 19: $1582-7$.

33. Napolitano A, Lowell BB, Damm D, Leibel RL, Ravussin E, Jimerson DC, Lesem MD, Van Dyke DC, Daly PA, Chatis $P$, et al. Concentrations of adipsin in blood and rates of adipsin secretion by adipose tissue in humans with normal, elevated and diminished adipose tissue mass. Int. J. Obes. Relat. Metab. Disord., 1994. 18: 213-8.

34. Ortega FJ, Moreno-Navarrete JM, Sabater M, Ricart W, Fruhbeck G, Fernandez-Real JM. Circulating glucagon is associated with inflammatory mediators in metabolically compromised subjects. Eur. J. Endocrinol., 2011. 165: 639-45.

35. Pomeroy C, Mitchell J, Eckert E, Raymond N, Crosby R, Dalmasso AP. Effect of body weight and caloric restriction on serum complement proteins, including Factor D/adipsin: studies in anorexia nervosa and obesity. Clin. Exp. Immunol., 1997. 108: 507-15.

36. Hernandez-Mijares A, Banuls C, Bellod L, Jover A, Sola E, Morillas C, Victor VM, Rocha M. Effect of weight loss on C3 and C4 components of complement in obese patients. Eur. J. Clin. Invest., 2011.

37. Onat A, Can G, Rezvani R, Cianflone K. Complement C3 and cleavage products in cardiometabolic risk. Clin. Chim. Acta, 2011. 412: 1171-9.

38. Oberbach A, Bluher $M$, Wirth $H$, Till H, Kovacs $P$, Kullnick $Y$, Schlichting $N$, Tomm JM, Rolle-Kampczyk $U$, Murugaiyan J, Binder H, Dietrich A, von Bergen M. Combined proteomic and metabolomic profiling of serum reveals association of the complement system with obesity and identifies novel markers of body fat mass changes. J. Proteome Res., 2011. 10: 4769-88.

39. van Dijk SJ, Feskens EJ, Heidema AG, Bos MB, van de Rest O, Geleijnse JM, de Groot LC, Muller M, Afman LA. Plasma protein profiling reveals protein clusters related to BMI and insulin levels in middle-aged overweight subjects. PLOS ONE, 2010. 5: e14422.

40. Hermsdorff HH, Zulet MA, Puchau B, Martinez JA. Central adiposity rather than total adiposity measurements are specifically involved in the inflammatory status from healthy young adults. Inflammation, 2011. 34: 161-70.

41. Blogowski W, Budkowska M, Salata D, Serwin K, Dolegowska B, Lokaj M, Prowans P, Starzynska T. Clinical analysis of selected complement-derived molecules in human adipose tissue. J. Transl. Med., 2013. 11: 11.

42. Weyer C, Tataranni PA, Pratley RE. Insulin action and insulinemia are closely related to the fasting complement C3, but not acylation stimulating protein concentration. Diabetes Care, 2000. 23: 779-85.

43. Warnberg J, Nova E, Moreno LA, Romeo J, Mesana MI, Ruiz JR, Ortega FB, Sjostrom M, Bueno M, Marcos A. Inflammatory proteins are related to total and abdominal adiposity in a healthy adolescent population: the AVENA Study. Am. J. Clin. Nutr., 2006. 84: 505-12.

44. Gabrielsson BG, Johansson JM, Lonn M, Jernas M, Olbers T, Peltonen M, Larsson I, Lonn L, Sjostrom L, Carlsson B, Carlsson LM. High expression of complement components in omental adipose tissue in obese men. Obes. Res., 2003. 11: 699-708.

45. Moreno-Navarrete JM, Martinez-Barricarte R, Catalan V, Sabater M, Gomez-Ambrosi J, Ortega FJ, Ricart W, Bluher M, Fruhbeck G, Rodriguez de Cordoba S, Fernandez-Real JM. Complement factor H is expressed in adipose tissue in association with insulin resistance. Diabetes, 2010. 59: 200-9.

46. Xia Z, Cianflone K. Acylation-stimulating protein precursor proteins in adipose tissue in human obesity. Metabolism, 2003. 52: 1360-6.

47. Insenser M, Montes-Nieto R, Vilarrasa N, Lecube A, Simo R, Vendrell J, Escobar-Morreale HF. A nontargeted proteomic approach to the study of visceral and subcutaneous adipose tissue in human obesity. Mol. Cell. Endocrinol., 2012. 363: 10-9.

48. Montague CT, Prins JB, Sanders L, Zhang J, Sewter CP, Digby J, Byrne CD, O'Rahilly S. Depot-related gene expression in human subcutaneous and omental adipocytes. Diabetes, 1998. 47: 1384-91.

49. Corominola H, Conner L, Beavers LS, Gadski RA, Johnson D, Caro JF, Rafaeloff-Phail R. Identification of novel genes differentially expressed in omental fat of obese subjects and obese type 2 diabetic patients. Diabetes, 2001. 50: 2822-30.

50. Koistinen HA, Vidal H, Karonen S-L, Dusserre E, Vallier P, Koivisto VA, Ebeling P. Plasma Acylation Stimulating Protein Concentration and Subcutaneous Adipose Tissue C3 mRNA Expression in Nondiabetic and Type 2 Diabetic Men. Arterioscler. Thromb. Vasc. Biol., 2001. 21: 1034-1039.

51. Zhang J, Wright W, Bernlohr DA, Cushman SW, Chen X. Alterations of the classic pathway of complement in adipose tissue of obesity and insulin resistance. American Journal of Physiology. Endocrinology and Metabolism, 2007. 292: E1433-40. 
52. Samaras K, Botelho NK, Chisholm DJ, Lord RV. Subcutaneous and visceral adipose tissue gene expression of serum adipokines that predict type 2 diabetes. Obesity, 2010. 18: 884-9.

53. Ylitalo K, Nuotio I, Viikari J, Auwerx J, Vidal H, Taskinen MR. C3, hormone-sensitive lipase, and peroxisome proliferator-activated receptor gamma expression in adipose tissue of familial combined hyperlipidemia patients. Metabolism, 2002. 51: 664-70.

54. van Greevenbroek MM, Ghosh S, van der Kallen CJ, Brouwers MC, Schalkwijk CG, Stehouwer CD. Up-regulation of the complement system in subcutaneous adipocytes from nonobese, hypertriglyceridemic subjects is associated with adipocyte insulin resistance. J. Clin. Endocrinol. Metab., 2012. 97: 4742-52.

55. Wlazlo N, van Greevenbroek MM, Ferreira I, Jansen EJ, Feskens EJ, van der Kallen CJ, Schalkwijk CG, Bravenboer B, Stehouwer CD. Low-grade inflammation and insulin resistance independently explain substantial parts of the association between body fat and serum C3: the CODAM study. Metabolism, 2012. 61: 1787-96.

56. Hillian AD, McMullen MR, Sebastian BM, Roychowdhury S, Kashyap SR, Schauer PR, Kirwan JP, Feldstein AE, Nagy LE. Mice lacking C1q are protected from high fat diet-induced hepatic insulin resistance and impaired glucose homeostasis. J. Biol. Chem., 2013. 288: 22565-75.

57. Phieler J, Chung KJ, Chatzigeorgiou A, Klotzsche-von Ameln A, Garcia-Martin R, Sprott D, Moisidou M, Tzanavari T, Ludwig B, Baraban E, Ehrhart-Bornstein M, Bornstein SR, Mziaut H, Solimena M, Karalis KP, Economopoulou M, Lambris JD, Chavakis T. The complement anaphylatoxin c5a receptor contributes to obese adipose tissue inflammation and insulin resistance. J. Immunol., 2013. 191: 4367-74.

58. Mamane Y, Chung Chan C, Lavallee G, Morin N, Xu LJ, Huang J, Gordon R, Thomas W, Lamb J, Schadt EE, Kennedy BP, Mancini JA. The C3a anaphylatoxin receptor is a key mediator of insulin resistance and functions by modulating adipose tissue macrophage infiltration and activation. Diabetes, 2009. 58: 2006-17.

59. Cianflone K, Xia Z, Chen LY. Critical review of acylation-stimulating protein physiology in humans and rodents. Biochim. Biophys. Acta, 2003. 1609: 127-43.

60. van Oostrom AJ, van Wijk J, Cabezas MC. Lipaemia, inflammation and atherosclerosis: novel opportunities in the understanding and treatment of atherosclerosis. Drugs, 2004. 64 Suppl 2: 19-41.

61. Maslowska M, Wang HW, Cianflone K. Novel roles for acylation stimulating protein/C3adesArg: a review of recent in vitro and in vivo evidence. Vitam. Horm., 2005. 70: 309-32.

62. Cianflone K. Acylation stimulating protein and the adipocyte. J. Endocrinol., 1997. 155: 203-6.

63. Paglialunga S, Fisette A, Yan Y, Deshaies Y, Brouillette JF, Pekna M, Cianflone K. Acylation-stimulating protein deficiency and altered adipose tissue in alternative complement pathway knockout mice. Am. J. Physiol. Endocrinol. Metab., 2008. 294: E521-9.

64. Gauvreau D, Roy C, Tom FQ, Lu H, Miegueu P, Richard D, Song WC, Stover C, Cianflone K. A new effector of lipid metabolism: Complement factor properdin. Mol. Immunol., 2012. 51: 73-81.

65. Engstrom G, Hedblad B, Janzon L, Lindgarde F. Weight gain in relation to plasma levels of complement factor 3: results from a population-based cohort study. Diabetologia, 2005. 48: 2525-31.

66. Hoyem PH, Bruun JM, Pedersen SB, Thiel S, Richelsen B, Christiansen JS, Hansen TK. The effect of weight loss on serum mannose-binding lectin levels. Clin. Dev. Immunol., 2012. 2012: 354894.

67. Mellbin LG, Hamsten A, Malmberg K, Steffensen R, Ryden L, Ohrvik J, Hansen TK. Mannose-binding lectin genotype and phenotype in patients with type 2 diabetes and myocardial infarction: a report from the DIGAMI 2 trial. Diabetes Care, 2010. 33: 2451-6.

68. Mellbin LG, Bjerre M, Thiel S, Hansen TK. Complement activation and prognosis in patients with type 2 diabetes and myocardial infarction: a report from the DIGAMI 2 trial. Diabetes Care, 2012. 35: 911-7.

69. Frauenknecht V, Thiel S, Storm L, Meier N, Arnold M, Schmid JP, Saner H, Schroeder V. Plasma levels of mannan-binding lectin (MBL)-associated serine proteases (MASPs) and MBL-associated protein in cardio- and cerebrovascular diseases. Clin. Exp. Immunol., 2013. 173: 112-20.

70. Rehrer CW, Karimpour-Fard A, Hernandez TL, Law CK, Stob NR, Hunter LE, Eckel RH. Regional differences in subcutaneous adipose tissue gene expression. Obesity, 2012. 20: 2168-73.

71. Diawara MR, Hue C, Wilder SP, Venteclef N, Aron-Wisnewsky J, Scott J, Clement K, Gauguier D, Calderari S. Adaptive expression of microRNA-125a in adipose tissue in response to obesity in mice and men. PLoS ONE, 2014. 9: e91375.

72. Alvarez-Llamas G, Szalowska E, de Vries MP, Weening D, Landman K, Hoek A, Wolffenbuttel BH, Roelofsen H, Vonk RJ. Characterization of the human visceral adipose tissue secretome. Mol. Cell. Proteomics, 2007. 6: 589600.

73. Zhong J, Krawczyk SA, Chaerkady R, Huang H, Goel R, Bader JS, Wong GW, Corkey BE, Pandey A. Temporal profiling of the secretome during adipogenesis in humans. J. Proteome Res., 2010. 9: 5228-38.

74. Kim J, Choi YS, Lim S, Yea K, Yoon JH, Jun DJ, Ha SH, Kim JW, Kim JH, Suh PG, Ryu SH, Lee TG. Comparative analysis of the secretory proteome of human adipose stromal vascular fraction cells during adipogenesis. Proteomics, 2010. 10: 394-405. 
75. Zvonic S, Lefevre M, Kilroy G, Floyd ZE, DeLany JP, Kheterpal I, Gravois A, Dow R, White A, Wu X, Gimble JM. Secretome of primary cultures of human adipose-derived stem cells: modulation of serpins by adipogenesis. Mol. Cell. Proteomics, 2007. 6: 18-28.

76. Soronen J, Laurila PP, Naukkarinen J, Surakka I, Ripatti S, Jauhiainen M, Olkkonen VM, Yki-Jarvinen H. Adipose tissue gene expression analysis reveals changes in inflammatory, mitochondrial respiratory and lipid metabolic pathways in obese insulin-resistant subjects. BMC Med. Genomics, 2012. 5: 9.

77. MacLaren RE, Cui W, Lu H, Simard S, Cianflone K. Association of adipocyte genes with ASP expression: a microarray analysis of subcutaneous and omental adipose tissue in morbidly obese subjects. BMC Med. Genomics, 2010. 3: 3.

78. Gupta A, Rezvani R, Lapointe M, Poursharifi P, Marceau P, Tiwari S, Tchernof A, Cianflone K. Downregulation of Complement C3 and C3aR Expression in Subcutaneous Adipose Tissue in Obese Women. PLoS ONE, 2014. 9: e95478.

79. Dahlman I, Elsen M, Tennagels N, Korn M, Brockmann B, Sell H, Eckel J, Arner P. Functional annotation of the human fat cell secretome. Arch. Physiol. Biochem., 2012. 118: 84-91.

80. Engstrom G, Hedblad B, Eriksson KF, Janzon L, Lindgarde F. Complement C3 is a risk factor for the development of diabetes: a population-based cohort study. Diabetes, 2005. 54: 570-5.

81. Keller TT, van Leuven SI, Meuwese MC, Wareham NJ, Luben R, Stroes ES, Hack CE, Levi M, Khaw KT, Boekholdt $\mathrm{SM}$. Serum levels of mannose-binding lectin and the risk of future coronary artery disease in apparently healthy men and women. Arterioscler. Thromb. Vasc. Biol., 2006: 26: 2345-50.

82. Luc G, Empana JP, Morange P, Juhan-Vague I, Arveiler D, Ferrieres J, Amouyel P, Evans A, Kee F, Bingham A, Machez E, Ducimetiere P. Adipocytokines and the risk of coronary heart disease in healthy middle aged men: the PRIME Study. Int. J. Obes. (Lond), 2010: 34: 118-26.

83. Onat A, Hergenc G, Can G, Kaya Z, Yuksel H. Serum complement C3: a determinant of cardiometabolic risk, additive to the metabolic syndrome, in middle-aged population. Metabolism, 2010: 59: 628-34.

84. Kriketos AD, Greenfield JR, Peake PW, Furler SM, Denyer GS, Charlesworth JA, Campbell LV. Inflammation, insulin resistance, and adiposity: a study of first-degree relatives of type 2 diabetic subjects. Diabetes Care, 2004: 27: 2033-40.

85. Chae YM, Park JK. The relationship between brachial ankle pulse wave velocity and complement 1 inhibitor. J. Korean Med. Sci., 2009: 24: 831-6.

86. Martin M, Gottsater A, Nilsson PM, Mollnes TE, Lindblad B, Blom AM. Complement activation and plasma levels of C4b-binding protein in critical limb ischemia patients. J. Vasc. Surg., 2009: 50: 100-6.

87. Somani R, Richardson VR, Standeven KF, Grant PJ, Carter AM. Elevated Properdin and Enhanced Complement Activation in First-Degree Relatives of South Asian Subjects With Type 2 Diabetes. Diabetes Care, 2012. 35: 894-9.

88. Hertle E, van Greevenbroek MM, Arts IC, van der Kallen CJ, Feskens EJ, Schalkwijk CG, Stehouwer CD. Complement activation products $\mathrm{C} 5 \mathrm{a}$ and sC5b-9 are associated with low-grade inflammation and endothelial dysfunction, but not with atherosclerosis in a cross-sectional analysis: The CODAM study. Int. J. Cardiol., 2014.

89. Hertle E, van Greevenbroek MM, Arts IC, van der Kallen CJ, Geijselaers SL, Feskens EJ, Jansen EH, Schalkwijk CG, Stehouwer CD. Distinct associations of complement C3a and its precursor C3 with atherosclerosis and cardiovascular disease. The CODAM study. Thromb. Haemost., 2014: 111: 1102-11.

90. Wallace TM, Levy JC, Matthews DR. Use and abuse of HOMA modeling. Diabetes Care, 2004: 27: $1487-95$.

91. Hernandez-Mijares A, Jarabo-Bueno MM, Lopez-Ruiz A, Sola-Izquierdo E, Morillas-Arino C, Martinez-Triguero ML. Levels of C3 in patients with severe, morbid and extreme obesity: its relationship to insulin resistance and different cardiovascular risk factors. Int. J. Obes. (Lond), 2007: 31: 927-32.

92. Yang Y, Lu HL, Zhang J, Yu HY, Wang HW, Zhang MX, Cianflone K. Relationships among acylation stimulating protein, adiponectin and complement C3 in lean vs obese type 2 diabetes. Int. J. Obes. (Lond), 2006: 30: 439-46.

93. Martinez-Gomez D, Eisenmann JC, Warnberg J, Gomez-Martinez S, Veses A, Veiga OL, Marcos A. Associations of physical activity, cardiorespiratory fitness and fatness with low-grade inflammation in adolescents: the AFINOS Study. Int. J. Obes., 2010: 34: 1501-7.

94. Phillips CM, Kesse-Guyot E, Ahluwalia N, McManus R, Hercberg S, Lairon D, Planells R, Roche HM. Dietary fat, abdominal obesity and smoking modulate the relationship between plasma complement component 3 concentrations and metabolic syndrome risk. Atherosclerosis, 2012: 220: 513-9.

95. De Pergola G, Tartagni M, Bartolomeo N, Bruno I, Masiello M, Caccavo D, Bavaro S, Silvestris F. Possible direct influence of complement 3 in decreasing insulin sensitvity in a cohort of overweight and obese subjects. Endocr. Metab. Immune Disord. Drug Targets, 2013: 13: 301-5.

96. Muscari A, Antonelli S, Bianchi G, Cavrini G, Dapporto S, Ligabue A, Ludovico C, Magalotti D, Poggiopollini G, Zoli M. Serum C3 is a stronger inflammatory marker of insulin resistance than C-reactive protein, leukocyte count, and erythrocyte sedimentation rate: comparison study in an elderly population. Diabetes Care, 2007: 30: $2362-8$. 
97. van Oostrom AJ, Alipour A, Plokker TW, Sniderman AD, Cabezas MC. The metabolic syndrome in relation to complement component 3 and postprandial lipemia in patients from an outpatient lipid clinic and healthy volunteers. Atherosclerosis, 2007: 190: 167-73.

98. Ursini F, Grembiale A, Naty S, Grembiale RD. Serum complement C3 correlates with insulin resistance in never treated psoriatic arthritis patients. Clin. Rheumatol., 2014: 33: 1759-64.

99. Wang B, Li Q, Jiang Y, Liu Z, Zhong L, Luo R, Cheng Q, Qing H. Serum complement C3 has a stronger association with insulin resistance than high sensitive C-reactive protein in non-diabetic Chinese. Inflamm. Res., 2011: 60: 63-8.

100. Wlazlo N, van Greevenbroek MM, Ferreira I, Feskens EJ, van der Kallen CJ, Schalkwijk CG, Bravenboer B, Stehouwer CD. Complement factor 3 is associated with insulin resistance and with incident type 2 diabetes over a 7-year follow-up period: the CODAM Study. Diabetes Care, 2014: 37: 1900-9.

101. Muscari A, Massarelli G, Bastagli L, Poggiopollini G, Tomassetti V, Drago G, Martignani C, Pacilli P, Boni P, Puddu P. Relationship of serum C3 to fasting insulin, risk factors and previous ischaemic events in middle-aged men. Eur. Heart J., 2000: 21: 1081-90.

102. Yesilova Z, Ozata M, Oktenli C, Bagci S, Ozcan A, Sanisoglu SY, Uygun A, Yaman H, Karaeren N, Dagalp K. Increased acylation stimulating protein concentrations in nonalcoholic fatty liver disease are associated with insulin resistance. Am. J. Gastroenterol., 2005: 100: 842-9.

103. Bjerre M, Kistorp C, Hansen TK, Faber J, Lip GY, Hildebrandt P, Flyvbjerg A. Complement activation, endothelial dysfunction, insulin resistance and chronic heart failure. Scand. Cardiovasc. J., 2010: 44: 260-6.

104. Labruna G, Pasanisi F, Nardelli C, Caso R, Vitale DF, Contaldo F, Sacchetti L. High leptin/adiponectin ratio and serum triglycerides are associated with an "at-risk" phenotype in young severely obese patients. Obesity (Silver Spring), 2011: 19: 1492-6.

105. Peake PW, Kriketos AD, Campbell LV, Charlesworth JA. Response of the alternative complement pathway to an oral fat load in first-degree relatives of subjects with type II diabetes. Int. J. Obes. (Lond), 2005: 29: 429-35.

106. Manco M, Fernandez-Real JM, Equitani F, Vendrell J, Valera Mora ME, Nanni G, Tondolo V, Calvani M, Ricart W, Castagneto $M$, Mingrone $G$. Effect of massive weight loss on inflammatory adipocytokines and the innate immune system in morbidly obese women. J. Clin. Endocrinol. Metab., 2007: 92: 483-90.

107. Fernandez-Real JM, Straczkowski M, Vendrell J, Soriguer F, Perez Del Pulgar S, Gallart L, Lopez-Bermejo A, Kowalska I, Manco M, Cardona F, Garcia-Gil MM, Mingrone G, Richart C, Ricart W, Zorzano A. Protection from inflammatory disease in insulin resistance: the role of mannan-binding lectin. Diabetologia, 2006: 49: 2402-11.

108. Ylitalo K, Pajukanta P, Meri S, Cantor RM, Mero-Matikainen N, Vakkilainen J, Nuotio I, Taskinen MR. Serum C3 but not plasma acylation-stimulating protein is elevated in Finnish patients with familial combined hyperlipidemia. Arterioscler. Thromb. Vasc. Biol., 2001: 21: 838-43.

109. Ohsawa I, Inoshita H, Ishii M, Kusaba G, Sato N, Mano S, Onda K, Gohda T, Horikoshi S, Ohi H, Tomino Y. Metabolic impact on serum levels of complement component 3 in Japanese patients. J. Clin. Lab. Anal., 2010: 24: 113-8.

110. Ajjan R, Carter AM, Somani R, Kain K, Grant PJ. Ethnic differences in cardiovascular risk factors in healthy Caucasian and South Asian individuals with the metabolic syndrome. J. Thromb. Haemost., 2007: 5: 754-60.

111. Phillips CM, Goumidi L, Bertrais S, Ferguson JF, Field MR, Kelly ED, Peloso GM, Cupples LA, Shen J, Ordovas JM, McManus R, Hercberg S, Portugal H, Lairon D, Planells R, Roche HM. Complement component 3 polymorphisms interact with polyunsaturated fatty acids to modulate risk of metabolic syndrome. Am. J. Clin. Nutr., 2009: 90: 1665-73.

112. Meijssen S, van Dijk H, Verseyden C, Erkelens DW, Cabezas MC. Delayed and exaggerated postprandial complement component 3 response in familial combined hyperlipidemia. Arterioscler. Thromb. Vasc. Biol., 2002: 22: 811-6.

113. Charlesworth JA, Peake PW, Campbell LV, Pussell BA, O'Grady S, Tzilopoulos T. The influence of oral lipid loads on acylation stimulating protein (ASP) in healthy volunteers. Int. J. Obes. Relat. Metab. Disord., 1998: 22: 1096102.

114. van Oostrom AJ, van Dijk H, Verseyden C, Sniderman AD, Cianflone K, Rabelink TJ, Castro Cabezas M. Addition of glucose to an oral fat load reduces postprandial free fatty acids and prevents the postprandial increase in complement component 3. Am. J. Clin. Nutr., 2004: 79: 510-5.

115. Alipour A, van Oostrom AJ, Van Wijk JP, Verseyden C, Plokker HW, Jukema JW, Rabelink AJ, Castro Cabezas M. Mannose binding lectin deficiency and triglyceride-rich lipoprotein metabolism in normolipidemic subjects. Atherosclerosis, 2009: 206: 444-50.

116. Halkes CJ, van Dijk H, de Jaegere PP, Plokker HW, van Der Helm Y, Erkelens DW, Castro Cabezas M. Postprandial increase of complement component 3 in normolipidemic patients with coronary artery disease: effects of expanded-dose simvastatin. Arterioscler. Thromb. Vasc. Biol., 2001: 21: 1526-30.

117. Fujita T, Hemmi S, Kajiwara M, Yabuki M, Fuke Y, Satomura A, Soma M. Complement-mediated chronic inflammation is associated with diabetic microvascular complication. Diabetes Metab. Res. Rev., 2013. 29: 220-6. 
118. Mantov S, Raev D. Additive effect of diabetes and systemic hypertension on the immune mechanisms of atherosclerosis. Int. J. Cardiol., 1996: 56: 145-8.

119. Siezenga MA, Chandie Shaw PK, van der Geest RN, Mollnes TE, Daha MR, Rabelink TJ, Berger SP. Enhanced complement activation is part of the unfavourable cardiovascular risk profile in South Asians. Clin. Exp. Immunol., 2009: 157: 98-103.

120. Raynor LA, Pankow JS, Duncan BB, Schmidt MI, Hoogeveen RC, Pereira MA, Young JH, Ballantyne CM. Novel risk factors and the prediction of type 2 diabetes in the Atherosclerosis Risk in Communities (ARIC) study. Diabetes Care, 2013: 36: 70-6.

121. Figueredo A, Ibarra JL, Bagazgoitia J, Rodriguez A, Molino AM, Fernandez-Cruz A, Patino R. Plasma C3d levels and ischemic heart disease in type II diabetes. Diabetes Care, 1993: 16: 445-9.

122. Kramer U, Herder C, Sugiri D, Strassburger K, Schikowski T, Ranft U, Rathmann W. Traffic-related air pollution and incident type 2 diabetes: results from the SALIA cohort study. Environ. Health Perspect., 2010: 118: 1273-9.

123. Karlsson E, Shaat N, Groop L. Can complement factors 5 and 8 and transthyretin be used as biomarkers for MODY 1 (HNF4A-MODY) and MODY 3 (HNF1A-MODY)? Diabet. Med., 2008: 25: 788-91.

124. Ebeling P, Teppo AM, Koistinen HA, Koivisto VA. Concentration of the complement activation product, acylation-stimulating protein, is related to C-reactive protein in patients with type 2 diabetes. Metabolism, 2001: 50: 283-7.

125. Ebeling P, Teppo AM, Koistinen HA, Viikari J, Ronnemaa T, Nissen M, Bergkulla S, Salmela P, Saltevo J, Koivisto VA. Troglitazone reduces hyperglycaemia and selectively acute-phase serum proteins in patients with Type II diabetes. Diabetologia, 1999: 42: 1433-8.

126. Bellentani S, Scaglioni F, Marino M, Bedogni G. Epidemiology of non-alcoholic fatty liver disease. Dig. Dis., 2010: 28: 155-61.

127. Fabbrini E, Magkos F, Mohammed BS, Pietka T, Abumrad NA, Patterson BW, Okunade A, Klein S. Intrahepatic fat, not visceral fat, is linked with metabolic complications of obesity. Proc. Natl. Acad. Sci. U. S. A., 2009: 106: 15430-5.

128. Farrell GC, Larter CZ. Nonalcoholic fatty liver disease: from steatosis to cirrhosis. Hepatology, 2006: 43: S99S112.

129. Scaglioni F, Ciccia S, Marino M, Bedogni G, Bellentani S. ASH and NASH. Dig. Dis., 2011: 29: 202-10.

130. Gao B, Seki E, Brenner DA, Friedman S, Cohen JI, Nagy L, Szabo G, Zakhari S. Innate immunity in alcoholic liver disease. Am. J. Physiol. Gastrointest. Liver Physiol., 2011: 300: G516-25.

131. Qin X, Gao B. The complement system in liver diseases. Cell. Mol. Immunol., 2006: 3: 333-40.

132. van Greevenbroek MM, Jacobs M, van der Kallen CJ, Vermeulen VM, Jansen EH, Schalkwijk CG, Ferreira I, Feskens EJ, Stehouwer CD. The cross-sectional association between insulin resistance and circulating complement C3 is partly explained by plasma alanine aminotransferase, independent of central obesity and general inflammation (the CODAM study). Eur. J. Clin. Invest., 2011: 41: 372-9.

133. Onat A, Hergenc G, Karabulut A, Turkmen S, Dogan Y, Uyarel H, Can G, Sansoy V. Serum gamma glutamyltransferase as a marker of metabolic syndrome and coronary disease likelihood in nondiabetic middle-aged and elderly adults. Prev. Med., 2006: 43: 136-9.

134. Rensen SS, Slaats Y, Driessen A, Peutz-Kootstra CJ, Nijhuis J, Steffensen R, Greve JW, Buurman WA. Activation of the complement system in human nonalcoholic fatty liver disease. Hepatology, 2009: 50: 1809-17.

135. Sreekumar R, Rosado B, Rasmussen D, Charlton M. Hepatic gene expression in histologically progressive nonalcoholic steatohepatitis. Hepatology, 2003: 38: 244-51.

136. Yilmaz Y, Yonal O, Kurt R, Alahdab YO, Eren F, Ozdogan O, Celikel CA, Imeryuz N, Kalayci C, Avsar E. Serum levels of omentin, chemerin and adipsin in patients with biopsy-proven nonalcoholic fatty liver disease. Scand. J. Gastroenterol., 2011: 46: 91-7.

137. Strey CW, Markiewski M, Mastellos D, Tudoran R, Spruce LA, Greenbaum LE, Lambris JD. The proinflammatory mediators C3a and C5a are essential for liver regeneration. J. Exp. Med., 2003: 198: 913-23.

138. Xu R, Lin F, He J, Jin L, Zhang JY, Fu J, Liu H, Wang S, Zhang Z, Wang FS. Complement 5a stimulates hepatic stellate cells in vitro, and is increased in the plasma of patients with chronic hepatitis B. Immunology, 2013: 138: 228-34.

139. Hillebrandt S, Wasmuth HE, Weiskirchen R, Hellerbrand C, Keppeler H, Werth A, Schirin-Sokhan R, Wilkens G, Geier A, Lorenzen J, Kohl J, Gressner AM, Matern S, Lammert F. Complement factor 5 is a quantitative trait gene that modifies liver fibrogenesis in mice and humans. Nat. Genet., 2005: 37: 835-43.

140. Bykov I, Jauhiainen M, Olkkonen VM, Saarikoski ST, Ehnholm C, Junnikkala S, Vakeva A, Lindros KO, Meri S. Hepatic gene expression and lipid parameters in complement C3(-/-) mice that do not develop ethanolinduced steatosis. J. Hepatol., 2007: 46: 907-14.

141. Bell LN, Lee L, Saxena R, Bemis KG, Wang M, Theodorakis JL, Vuppalanchi R, Alloosh M, Sturek M, Chalasani N. Serum proteomic analysis of diet-induced steatohepatitis and metabolic syndrome in the Ossabaw miniature swine. Am. J. Physiol. Gastrointest. Liver Physiol., 2010: 298: G746-54. 
142. Wlazlo N, van Greevenbroek MM, Ferreira I, Jansen EH, Feskens EJ, van der Kallen CJ, Schalkwijk CG, Bravenboer B, Stehouwer CD. Activated complement factor 3 is associated with liver fat and liver enzymes: the CODAM study. Eur. J. Clin. Invest., 2013: 43: 679-88.

143. Pritchard MT, McMullen MR, Stavitsky AB, Cohen JI, Lin F, Medof ME, Nagy LE. Differential contributions of C3, C5, and decay-accelerating factor to ethanol-induced fatty liver in mice. Gastroenterology, 2007: 132: 1117-26.

144. Roychowdhury S, McMullen MR, Pritchard MT, Hise AG, van Rooijen N, Medof ME, Stavitsky AB, Nagy LE. An early complement-dependent and TLR-4-independent phase in the pathogenesis of ethanol-induced liver injury in mice. Hepatology, 2009: 49: 1326-34.

145. Jarvelainen HA, Vakeva A, Lindros KO, Meri S. Activation of complement components and reduced regulator expression in alcohol-induced liver injury in the rat. Clin. Immunol., 2002: 105: 57-63.

146. Cohen JI, Roychowdhury S, McMullen MR, Stavitsky AB, Nagy LE. Complement and alcoholic liver disease: role of $\mathrm{C} 1 \mathrm{q}$ in the pathogenesis of ethanol-induced liver injury in mice. Gastroenterology, 2010: 139: 664-74, 74 e1.

147. Bongrazio M, Pries AR, Zakrzewicz A. The endothelium as physiological source of properdin: role of wall shear stress. Mol. Immunol., 2003: 39: 669-75.

148. Bossi F, Peerschke El, Ghebrehiwet B, Tedesco F. Cross-talk between the complement and the kinin system in vascular permeability. Immunol. Lett., 2011: 140: 7-13.

149. Oksjoki R, Kovanen PT, Pentikainen MO. Role of complement activation in atherosclerosis. Curr. Opin. Lipidol., 2003: 14: 477-82.

150. Bossi F, Fischetti F, Pellis V, Bulla R, Ferrero E, Mollnes TE, Regoli D, Tedesco F. Platelet-activating factor and kinin-dependent vascular leakage as a novel functional activity of the soluble terminal complement complex. J. Immunol., 2004: 173: 6921-7.

151. Speidl WS, Kastl SP, Huber K, Wojta J. Complement in atherosclerosis: friend or foe? J. Thromb. Haemost., 2011: 9: 428-40.

152. Torzewski M, Bhakdi S. Complement and atherosclerosis-united to the point of no return? Clin. Biochem., 2013: 46: 20-5.

153. Haskard DO, Boyle JJ, Mason JC. The role of complement in atherosclerosis. Curr. Opin. Lipidol., 2008: 19: 478-82.

154. Cao W, Bobryshev YV, Lord RS, Oakley RE, Lee SH, Lu J. Dendritic cells in the arterial wall express C1q: potential significance in atherogenesis. Cardiovasc. Res., 2003: 60: 175-86.

155. Francescut L, Steiner T, Byrne S, Cianflone K, Francis S, Stover C. The role of complement in the development and manifestation of murine atherogenic inflammation: novel avenues. J. Innate Immun., 2012: 4: 260-72.

156. Ajjan R, Grant PJ, Futers TS, Brown JM, Cymbalista CM, Boothby M, Carter AM. Complement C3 and C-reactive protein levels in patients with stable coronary artery disease. Thromb. Haemost., 2005: 94: 1048-53.

157. Carter AM, Prasad UK, Grant PJ. Complement C3 and C-reactive protein in male survivors of myocardial infarction. Atherosclerosis, 2009: 203: 538-43.

158. Széplaki G, Prohászka Z, Duba J, Rugonfalvi-Kiss S, Karádi I, Kókai M, Kramer J, Füst G, Kleiber M, Romics L, Varga L. Association of high serum concentration of the third component of complement (C3) with preexisting severe coronary artery disease and new vascular events in women. Atherosclerosis, 2004: 177: 383-9.

159. Onat A, Uzunlar B, Hergenc G, Yazici M, Sari I, Uyarel H, Can G, Sansoy V. Cross-sectional study of complement C3 as a coronary risk factor among men and women. Clin. Sci. (Lond), 2005: 108: 129-35.

160. van Greevenbroek MM, Jacobs M, van der Kallen CJ, Blaak EE, Jansen EH, Schalkwijk CG, Feskens EJ, Stehouwer $\mathrm{CD}$. Human plasma complement C3 is independently associated with coronary heart disease, but only in heavy smokers (the CODAM study). Int. J. Cardiol., 2012: 154: 158-62.

161. Muscari A, Bozzoli C, Puddu GM, Sangiorgi Z, Dormi A, Rovinetti C, Descovich GC, Puddu P. Association of serum C3 levels with the risk of myocardial infarction. Am. J. Med., 1995: 98: 357-64.

162. Engstrom G, Hedblad B, Janzon L, Lindgarde F. Complement C3 and C4 in plasma and incidence of myocardial infarction and stroke: a population-based cohort study. Eur. J. Cardiovasc. Prev. Rehabil., 2007: 14: 392-7.

163. Engstrom G, Hedblad B, Berglund G, Janzon L, Lindgarde F. Plasma levels of complement C3 is associated with development of hypertension: a longitudinal cohort study. J. Hum. Hypertens., 2007: 21: 276-82.

164. Cavusoglu E, Eng C, Chopra V, Ruwende C, Yanamadala S, Clark LT, Pinsky DJ, Marmur JD. Usefulness of the serum complement component $\mathrm{C} 4$ as a predictor of stroke in patients with known or suspected coronary artery disease referred for coronary angiography. Am. J. Cardiol., 2007: 100: 164-8.

165. Adamsson Eryd S, Smith JG, Melander O, Hedblad B, Engstrom G. Inflammation-sensitive proteins and risk of atrial fibrillation: a population-based cohort study. Eur. J. Epidemiol., 2011: 26: 449-55.

166. Muscari A, Martignani C, Bastagli L, Poggiopollini G, Tomassetti V, Baldini L, Cappelletti O, Boni P, Ravaglia G, Puddu P. A comparison of acute phase proteins and traditional risk factors as markers of combined plaque and intima-media thickness and plaque density in carotid and femoral arteries. Eur. J. Vasc. Endovasc. Surg., 2003: 26: 81-7. 
167. De Pergola G, Ciccone MM, Guida P, Morea G, Giannuzzo E, Cortese F, Scicchitano P, Favale S, Silvestris F. Relationship between C3 Levels and Common Carotid Intima-Media Thickness in Overweight and Obese Patients. Obes. Facts, 2011: 4: 159-63.

168. Cianflone K, Zhang XJ, Genest J, Jr., Sniderman A. Plasma acylation-stimulating protein in coronary artery disease. Arterioscler. Thromb. Vasc. Biol., 1997: 17: 1239-44.

169. Kostner KM, Fahti RB, Case C, Hobson P, Tate J, Marwick TH. Inflammation, complement activation and endothelial function in stable and unstable coronary artery disease. Clin. Chim. Acta, 2006: 365: 129-34.

170. Donahue MP, Rose K, Hochstrasser D, Vonderscher J, Grass P, Chibout SD, Nelson CL, Sinnaeve P, GoldschmidtClermont PJ, Granger CB. Discovery of proteins related to coronary artery disease using industrial-scale proteomics analysis of pooled plasma. Am. Heart J., 2006: 152: 478-85.

171. Clark DJ, Cleman MW, Pfau SE, Rollins SA, Ramahi TM, Mayer C, Caulin-Glaser T, Daher E, Kosiborod M, Bell L, Setaro JF. Serum complement activation in congestive heart failure. Am. Heart J., 2001: 141: 684-90.

172. Yasuda M, Takeuchi K, Hiruma M, lida H, Tahara A, Itagane H, Toda I, Akioka K, Teragaki M, Oku H. The complement system in ischemic heart disease. Circulation, 1990: 81: 156-63.

173. Hoffmeister HM, Ehlers R, Buttcher E, Kazmaier S, Szabo S, Beyer ME, Steinmetz A, Seipel L. Comparison of Creactive protein and terminal complement complex in patients with unstable angina pectoris versus stable angina pectoris. Am. J. Cardiol., 2002: 89: 909-12.

174. Horvath Z, Csuka D, Vargova K, Kovacs A, Molnar AA, Gulacsi-Bardos P, Lee S, Varga L, Kiss RG, Preda I, Fust G. Elevated $\mathrm{C} 1 \mathrm{rC1sC1inh}$ levels independently predict atherosclerotic coronary heart disease. Mol. Immunol., 2013: 54: 8-13.

175. Videm V, Wiseth R, Gunnes S, Madsen HO, Garred P. Multiple inflammatory markers in patients with significant coronary artery disease. Int. J. Cardiol., 2007: 118: 81-7.

176. Hirata A, Kishida K, Nakatsuji H, Kobayashi H, Funahashi T, Shimomura I. High serum C1q-adiponectin/total adiponectin ratio correlates with coronary artery disease in Japanese type 2 diabetics. Metabolism, 2013: 62: 578-85.

177. Kosar F, Varol E, lleri M, Ayaz S, Hisar I, Kisacik H. Circulating cytokines and complements in chronic heart failure. Angiology, 1999: 50: 403-8.

178. Kimoto K, Inoue T, Oku K, Mori T, Kusuda M, Handa K, Sakata N, Sasaki J, Arakawa K. Relation of C4b-binding protein to athero-sclerosis of the descending thoracic aorta. Artery, 1996: 22: 101-14.

179. Best LG, Davidson M, North KE, MacCluer JW, Zhang Y, Lee ET, Howard BV, DeCroo S, Ferrell RE. Prospective analysis of mannose-binding lectin genotypes and coronary artery disease in American Indians: the Strong Heart Study. Circulation, 2004: 109: 471-5.

180. Saevarsdottir S, Oskarsson OO, Aspelund T, Eiriksdottir G, Vikingsdottir T, Gudnason V, Valdimarsson $H$. Mannan binding lectin as an adjunct to risk assessment for myocardial infarction in individuals with enhanced risk. J. Exp. Med., 2005: 201: 117-25.

181. Siezenga MA, Chandie Shaw PK, Daha MR, Rabelink TJ, Berger SP. Low Mannose-Binding Lectin (MBL) genotype is associated with future cardiovascular events in type 2 diabetic South Asians A prospective cohort study. Cardiovasc. Diabetol., 2011: 10: 60.

182. Vengen IT, Madsen HO, Garred P, Platou C, Vatten L, Videm V. Mannose-binding lectin deficiency is associated with myocardial infarction: the HUNT2 study in Norway. PLoS ONE, 2012: 7: e42113.

183. Troelsen LN, Garred P, Christiansen B, Torp-Pedersen C, Christensen IJ, Narvestad E, Jacobsen S. Double role of mannose-binding lectin in relation to carotid intima-media thickness in patients with rheumatoid arthritis. Mol. Immunol., 2010: 47: 713-8.

184. Aittoniemi J, Fan YM, Laaksonen R, Janatuinen T, Vesalainen R, Nuutila P, Knuuti J, Hulkkonen J, Hurme M, Lehtimaki $\mathrm{T}$. The effect of mannan-binding lectin variant alleles on coronary artery reactivity in healthy young men. Int. J. Cardiol., 2004: 97: 317-8.

185. Strack C, Baessler A, Wagner F, Bruxmeier J, Yaroslavskii O, Rousseva E, Loew T, Riegger G, Schmitz G, Fischer M. Mannose-binding lectin in obesity with different degrees of metabolic syndrome abnormalities: association with atherogenic and metabolic traits. J. Atheroscl. Thromb., 2012: 19: 539-51.

186. Alipour A, Cabezas MC, Elte JW, Vallve JC, Ribalta J, Zwinderman AH, Defesche JC, Jukema JW. Mannose binding lectin 2 haplotypes do not affect the progression of coronary atherosclerosis in men with proven coronary artery disease treated with pravastatin. Atherosclerosis, 2011: 215: 125-9.

187. Sofat R, Casas JP, Kumari M, Talmud PJ, Ireland H, Kivimaki M, Marmot M, Hughes AD, Thom S, Ebrahim S, Whittaker JC, Smeeth L, Lawlor DA, Humphries SE, Hingorani AD. Genetic variation in complement factor $\mathrm{H}$ and risk of coronary heart disease: eight new studies and a meta-analysis of around 48,000 individuals. Atherosclerosis, 2010: 213: 184-90.

188. Zhang HF, Wang JF, Wang Y, Zhu LG, Lei L. Complement factor H Y402H gene polymorphism and coronary heart disease susceptibility: a meta-analysis. Mol. Biol. Rep., 2011: 38: 2933-8. 
189. Prugger C, Luc G, Haas B, Arveiler D, Machez E, Ferrieres J, Ruidavets JB, Bingham A, Montaye M, Amouyel P, Yarnell J, Kee F, Ducimetiere P, Empana JP. Adipocytokines and the risk of ischemic stroke: the PRIME Study. Ann. Neurol., 2012: 71: 478-86.

190. Distelmaier K, Adlbrecht C, Jakowitsch J, Winkler S, Dunkler D, Gerner C, Wagner O, Lang IM, Kubicek M. Local complement activation triggers neutrophil recruitment to the site of thrombus formation in acute myocardial infarction. Thromb. Haemost., 2009: 102: 564-72.

191. Oksjoki R, Kovanen PT, Meri S, Pentikainen MO. Function and regulation of the complement system in cardiovascular diseases. Front. Biosci., 2007: 12: 4696-708.

192. Arumugam TV, Magnus T, Woodruff TM, Proctor LM, Shiels IA, Taylor SM. Complement mediators in ischemiareperfusion injury. Clin. Chim. Acta, 2006: 374: 33-45.

193. Holt CB, Thiel S, Munk K, Ostergaard JA, Botker HE, Hansen TK. Association between endogenous complement inhibitor and myocardial salvage in patients with myocardial infarction. Eur. Heart J. Acute Cardiovas. Care, 2014: 3: 3-9.

194. Lindberg S, Pedersen SH, Mogelvang R, Galatius S, Flyvbjerg A, Jensen JS, Bjerre M. Soluble form of membrane attack complex independently predicts mortality and cardiovascular events in patients with ST-elevation myocardial infarction treated with primary percutaneous coronary intervention. Am. Heart J., 2012: 164: 786-92.

195. Szeplaki G, Szegedi R, Hirschberg K, Gombos T, Varga L, Karadi I, Entz L, Szeplaki Z, Garred P, Prohaszka Z, Fust G. Strong complement activation after acute ischemic stroke is associated with unfavorable outcomes. Atherosclerosis, 2009: 204: 315-20.

196. Speidl WS, Exner M, Amighi J, Kastl SP, Zorn G, Maurer G, Wagner O, Huber K, Minar E, Wojta J, Schillinger M. Complement component $\mathrm{C5}$ a predicts future cardiovascular events in patients with advanced atherosclerosis. Eur. Heart J., 2005: 26: 2294-9.

197. Speidl WS, Exner M, Amighi J, Mlekusch W, Sabeti S, Kastl SP, Zorn G, Maurer G, Wagner O, Huber K, Minar E, Wojta J, Schillinger M. Complement component C5a predicts restenosis after superficial femoral artery balloon angioplasty. J. Endovasc. Ther., 2007: 14: 62-9.

198. Speidl WS, Katsaros KM, Kastl SP, Zorn G, Huber K, Maurer G, Wojta J, Christ G. Coronary late lumen loss of drug eluting stents is associated with increased serum levels of the complement components C3a and C5a. Atherosclerosis, 2010: 208: 285-9.

199. Szeplaki G, Varga L, Laki J, Dosa E, Madsen HO, Prohaszka Z, Szabo A, Acsady G, Selmeci L, Garred P, Fust G, Entz L. Elevated complement C3 is associated with early restenosis after eversion carotid endarterectomy. Thromb. Haemost., 2006: 96: 529-34.

200. Palikhe A, Sinisalo J, Seppanen M, Haario H, Meri S, Valtonen V, Nieminen MS, Lokki ML. Serum complement C3/C4 ratio, a novel marker for recurrent cardiovascular events. Am. J. Cardiol., 2007: 99: 890-5.

201. Cervera A, Planas AM, Justicia C, Urra X, Jensenius JC, Torres F, Lozano F, Chamorro A. Genetically-defined deficiency of mannose-binding lectin is associated with protection after experimental stroke in mice and outcome in human stroke. PLoS ONE, 2010: 5: e8433.

202. Shantikumar S, Grant PJ, Catto AJ, Bamford JM, Carter AM. Elevated C-reactive protein and long-term mortality after ischaemic stroke: relationship with markers of endothelial cell and platelet activation. Stroke, 2009: 40: 977-9.

203. Szeplaki G, Varga L, Laki J, Dosa E, Rugonfalvi-Kiss S, Madsen HO, Prohaszka Z, Kocsis A, Gal P, Szabo A, Acsady G, Karadi I, Selmeci L, Garred P, Fust G, Entz L. Low c1-inhibitor levels predict early restenosis after eversion carotid endarterectomy. Arterioscler. Thromb. Vasc. Biol., 2007: 27: 2756-62.

204. Gordon SM, Deng J, Lu LJ, Davidson WS. Proteomic characterization of human plasma high density lipoprotein fractionated by gel filtration chromatography. J. Proteome Res., 2010: 9: 5239-49.

205. Rezaee F, Casetta B, Levels JH, Speijer D, Meijers JC. Proteomic analysis of high-density lipoprotein. Proteomics, 2006: 6: 721-30.

206. Vaisar T, Pennathur S, Green PS, Gharib SA, Hoofnagle AN, Cheung MC, Byun J, Vuletic S, Kassim S, Singh P, Chea H, Knopp RH, Brunzell J, Geary R, Chait A, Zhao XQ, Elkon K, Marcovina S, Ridker P, Oram JF, Heinecke $\mathrm{JW}$. Shotgun proteomics implicates protease inhibition and complement activation in the antiinflammatory properties of HDL. J. Clin. Invest., 2007: 117: 746-56.

207. Parra S, Vives G, Ferre R, Gonzalez M, Guardiola M, Ribalta J, Castro A. Complement system and small HDL particles are associated with subclinical atherosclerosis in SLE patients. Atherosclerosis 2012: 225: 224-30.

208. Acosta J, Hettinga J, Fluckiger R, Krumrei N, Goldfine A, Angarita L, Halperin J. Molecular basis for a link between complement and the vascular complications of diabetes. Proc. Natl. Acad. Sci. U. S. A., 2000: 97: 5450-5.

209. Ghosh P, Vaidya A, Sahoo R, Goldfine A, Herring N, Bry L, Chorev M, Halperin JA. Glycation of the complement regulatory protein CD59 is a novel biomarker for glucose handling in humans. J. Clin. Endocrinol. Metab., 2014: 99: E999-E1006. 
210. Qin X, Goldfine A, Krumrei N, Grubissich L, Acosta J, Chorev M, Hays AP, Halperin JA. Glycation inactivation of the complement regulatory protein CD59: a possible role in the pathogenesis of the vascular complications of human diabetes. Diabetes, 2004: 53: 2653-61.

211. Hertle E, van Greevenbroek MM, Stehouwer CD. Complement C3: an emerging risk factor in cardiometabolic disease. Diabetologia, 2012: 55: 881-4.

212. El Khoudary SR, Shields KJ, Chen HY, Matthews KA. Menopause, complement, and hemostatic markers in women at midlife: the Study of Women's Health Across the Nation. Atherosclerosis, 2013: 231: 54-8.

213. Capuano V, D'Arminio T, La Sala G, Mazzotta G. The third component of the complement (C3) is a marker of the risk of atherogenesis. Eur. J. Cardiovasc. Prev. Rehabil., 2006: 13: 658-60.

214. Howes JM, Richardson VR, Smith KA, Schroeder V, Somani R, Shore A, Hess K, Ajjan R, Pease RJ, Keen JN, Standeven KF, Carter AM. Complement C3 is a novel plasma clot component with anti-fibrinolytic properties. Diab. Vasc. Dis. Res., 2012: 9: 216-25.

215. Schroeder V, Carter AM, Dunne J, Mansfield MW, Grant PJ. Proinflammatory and hypofibrinolytic phenotype in healthy first-degree relatives of patients with Type 2 diabetes. J. Thromb. Haemost., 2010: 8: 2080-2.

216. Hess K, Alzahrani SH, Mathai M, Schroeder V, Carter AM, Howell G, Koko T, Strachan MW, Price JF, Smith KA, Grant PJ, Ajjan RA. A novel mechanism for hypofibrinolysis in diabetes: the role of complement C3. Diabetologia, 2012: 55: 1103-13.

217. Puchau B, Zulet MA, de Echavarri AG, Hermsdorff HH, Martinez JA. Dietary total antioxidant capacity is negatively associated with some metabolic syndrome features in healthy young adults. Nutrition, 2010: 26: 534-41.

218. Puchau B, Zulet MA, Gonzalez de Echavarri A, Navarro-Blasco I, Martinez JA. Selenium intake reduces serum C3, an early marker of metabolic syndrome manifestations, in healthy young adults. Eur. J. Clin. Nutr., 2009: 63: 858-64.

219. van Greevenbroek MM, Arts IC, van der Kallen CJ, Dagnelie PC, Ferreira I, Jansen E, Schalkwijk CG, Feskens EJ, Stehouwer CD. Complement C3 is inversely associated with habitual intake of provitamin A but not with dietary fat, fatty acids, or vitamin $\mathrm{E}$ in middle-aged to older white adults and positively associated with intake of retinol in middle-aged to older white women. J. Nutr., 2014: 144: 61-7.

220. Artero EG, Espana-Romero V, Jimenez-Pavon D, Martinez-Gomez D, Warnberg J, Gomez-Martinez S, Gonzalez-Gross M, Vanhelst J, Kafatos A, Molnar D, De Henauw S, Moreno LA, Marcos A, Castillo MJ. Muscular fitness, fatness and inflammatory biomarkers in adolescents. Pediatr. Obes., 2014: 9: 391-400.

221. Ruiz JR, Ortega FB, Warnberg J, Moreno LA, Carrero JJ, Gonzalez-Gross M, Marcos A, Gutierrez A, Sjostrom M. Inflammatory proteins and muscle strength in adolescents: the Avena study. Arch. Pediatr. Adolesc. Med., 2008: 162: 462-8.

222. Altunbas H, Karayalcin U, Undar L. Glycemic control and coagulation inhibitors in diabetic patients. Haemostasis, 1998: 28: 307-12.

223. Muscari A, Bastagi L, Poggiopollini G, Tomassetti V, Massarelli G, Boni P, Puddu P. Short term effect of atorvastatin and vitamin $E$ on serum levels of $C 3$, a sensitive marker of the risk of myocardial infarction in men. Cardiovasc. Drugs Ther., 2001: 15: 453-8.

224. Rajzer M, Wojciechowska W, Kawecka-Jaszcz K, Undas A. Plasma fibrin clot properties in arterial hypertension and their modification by antihypertensive medication. Thromb. Res., 2012: 130: 99-103.

225. Oktenli C, Ozgurtas T, Dede M, Sanisoglu YS, Yenen MC, Yesilova Z, Kenar L, Kurt YG, Baser I, Smith J, Cianflone K. Metformin decreases circulating acylation-stimulating protein levels in polycystic ovary syndrome. Gynecol. Endocrinol., 2007: 23: 710-5. 


\section{Chapter 3 CHobfGr 3}

Complement C3: an emerging risk factor in cardiometabolic disease

Diabetologia, 2012. 55: 881-4

E. Hertle, M.M.J. van Greevenbroek, C.D.A Stehouwer 


\section{Abstract}

Complement C3 is the central component of the complement system and activation of C3 via either of the three major activation pathways - the classical, the lectin and the alternative pathway - results in initiation of the terminal complement pathway and release of the anaphylatoxin C3a. Both terminal pathway activation and signalling of C3a and its inactivation product $\mathrm{C3a}$-desarg via the $\mathrm{C} 3 \mathrm{a}$-receptor and the $\mathrm{C} 5 \mathrm{a}$-like receptor 2 , respectively, can induce inflammatory, immunomodulatory and metabolic responses. C3 has been implicated in metabolic disorders, notably adiposity, dyslipidaemia, insulin resistance, liver dysfunction and diabetes, and complement C3 is increasingly recognised as cardiometabolic risk factor. Complement C3 may play a role in the macrovascular as well as the microvascular complications of diabetes. Moreover, C3 may interact with the coagulation system and as such also contribute to a procoagulant, hypofibrinolytic and ultimately prothrombotic state. Recent data suggested a diabetes-dependent incorporation of C3 into fibrin clots, with concomitant effects on clot characteristics. Taken together, epidemiological and experimental evidence concordantly point to a role of complement C3 in metabolic, atherosclerotic/atherothrombotic and microangiopathic processes and further research should be directed towards the elucidation of complement function and activation in cardiometabolic disorders. 


\section{Commentary}

The complement system, a complex protein network initially identified as part of the innate immune system, is increasingly recognised as an essential regulator of cell and tissue homeostasis. It consists of soluble and membrane-bound proteins functioning in cascades of stepwise protease activation; effector functions include the release of anaphylatoxins and formation of terminal complement complexes (TCCS). Anaphylatoxins operate by binding to their receptors on a variety of immune and non-immune cells, where they exert proinflammatory, immunomodulatory and metabolic effects. TCCs are assembled upon membranes, where they exert stimulatory effects on cell cycle and cell metabolism (sublytic TCCs) or promote cytolysis when present in higher amounts (lytic TCCs, also referred to as membrane-attack complexes [MACs]).

C3 lies at the heart of the complement network, as all three major activation pathways may result in cleavage of $\mathrm{C} 3$ and initiation of the downstream terminal pathway. Systemic levels of C3 may reflect the potential for complement activation. Upon activation of C3, C3a and C3b are generated. Once formed, the anaphylatoxin C3a is rapidly desarginated by a carboxypeptidase, generating C3a-desarg. Although this was previously thought to be an inactivation process, $\mathrm{C} 3 \mathrm{a}$-desArg has been recognised as a lipogenic hormone and is now also known as acylation-stimulating protein (ASP) [1]. C3b is instrumental in the activation of the terminal pathway of complement activation, which leads to formation of TCCs/MACs (figure 1).

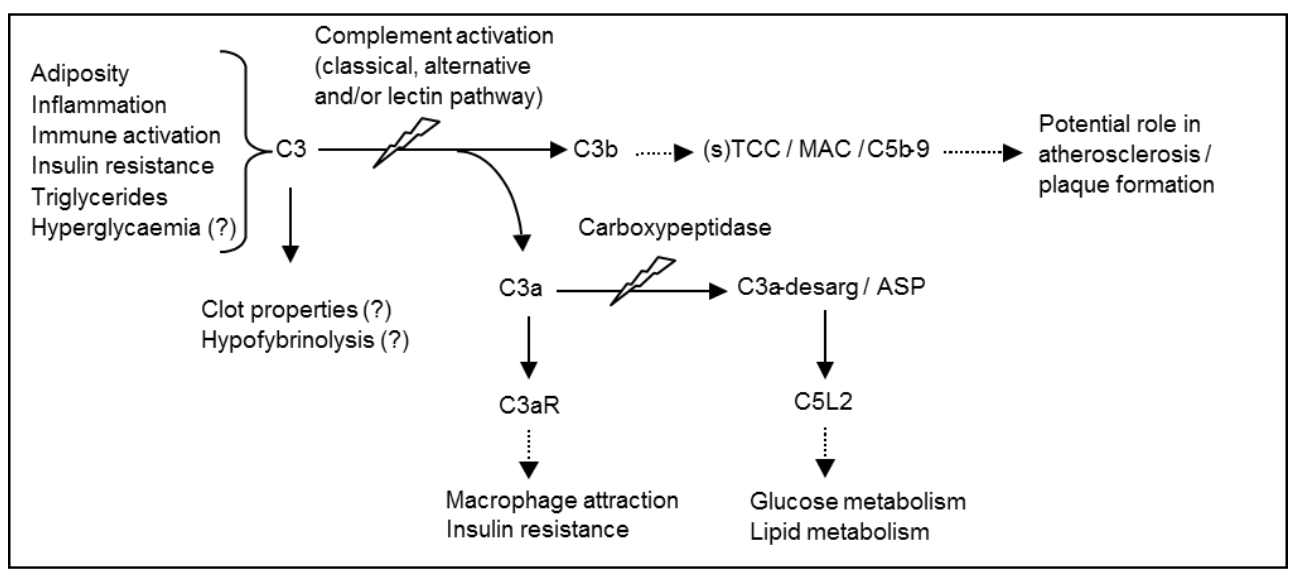

Figure 1: Adiposity, inflammation, immune activation, insulin resistance, hypertriglyceridaemia and, potentially, hyperglycaemia may lead to increased systemic levels of C3. C3a and C3b are generated upon activation of C3. C3b is part of the multi-step complement activation cascade that eventually leads to the formation of (soluble) TCCs/MACs. The anaphylatoxin C3a is rapidly degraded into its desarginated form, which is also known as ASP. Both C3a and C3adesarg/ASP can, via binding to their respective receptors (C3aR and C5L2), exert relevant effects with respect to diabetes and CVD. The solid arrows denote direct effects, the dotted arrows denote more distal effects and the lightning bolts indicate activation. 
Recent data suggest that C3 plays a role in metabolic disorders. For example, a high systemic concentration of C3 was shown to be independently associated with the incidence of type 2 diabetes, at least in men [2]. Additionally, systemic C3 levels have been associated with several diabetes risk factors, including adiposity (waist, BMI), serum triacylglycerol and insulin resistance (as reviewed in [3]). Plasma C3 levels are also higher in non-alcoholic fatty liver disease (NAFLD) [4], a common comorbidity associated with obesity and in type 2 diabetes, and possibly also in type 1 diabetes; in agreement with this observation, alanine aminotransferase - a marker of liver dysfunction-was also associated with plasma levels of C3 [5]. The link between systemic C3 and adiposity is substantiated by the observations that adipose tissue secretes $\mathrm{C} 3$, that weight gain is associated with an increase in C3 and that C3 decreases upon weight loss [3]. Further evidence supporting a potential role for C3 in (the development of) diabetes and associated metabolic disorders is the strong association of C3, which is an acute-phase reactant, with inflammatory markers [6]. In addition, complement activation can promote systemic inflammation [7].

At least two signalling pathways have been identified that may contribute to the association of $\mathrm{C} 3$ activation with insulin resistance, adipose tissue function and lipid metabolism. First, expression of the $\mathrm{C} 3 \mathrm{a}$ receptor $(\mathrm{C} 3 \mathrm{aR})$ is particularly high in white adipose tissue and is strongly upregulated after a high-fat diet [8]. Interruption of the C3-C3a-C3aR axis in a $\mathrm{C} \mathrm{aR}^{-/-}$mouse model prevented diet-induced insulin resistance [8]. Second, interruption of the C3-(C3a)-C3a-desarg/ASP-C5a-like receptor 2 (C5L2) pathway in a C5L2-- mouse model had multiple metabolic effects, including reduced triacylglycerol synthesis in adipose tissue and delayed triacylglycerol and glucose clearance [9]. Reduced signalling of ASP via C5L2 (ASP resistance) may contribute to altered adipose tissue metabolism in obesity/insulin resistance phenotypes in humans [10]. Overall, substantial evidence points towards an active role of C3, at least partly via C3 activation, in diabetes-related metabolic diseases. However, the causal relationships and order of the events that occur in the vicious cycles of adiposity, insulin resistance and inflammation, remain to be unravelled.

C3 may also play a role in the cardiovascular complications of diabetes and related metabolic diseases. C3 has repeatedly been shown to be associated with different manifestations of prevalent and incident cardiovascular disease (CVD), often - but not always - independent of classical risk factors [3]. The observation that the associations with CVD risk factors and disease manifestations are not uniform suggests that there may be distinct properties of C3 contributing to the development of CVD. Mechanistically, the involvement of C3 in the atherosclerotic process is illustrated by the presence of $\mathrm{C} 3$ and its activation products in atherosclerotic plaques. A causal role was deduced from animal studies showing that deposition of lipids and complement in the intima precedes the infiltration of inflammatory cells and foam cell development [11].

The role of complement activation in diabetic vascular complications may not be confined to macrovascular disease. The complement system may also contribute to compromised microvascular function, and activation of complement, including C3, may thus contribute to the diabetic nephropathy, retinopathy and neuropathy. Its role in nephropathy is supported by the presence of activated C3 in glomeruli and glomerular capillaries of animal models of 
type 1 and type 2 diabetes $[12,13]$. Furthermore, C3 activation may aggravate existing kidney disease since non-selective filtration of complement proteins may result in intratubular complement activation and tubular damage $[14,15]$. Support for a role of C3 in retinopathy and neuropathy is provided by data indicating that activated C3 and TCC/MAC (representing terminal pathway activation) are present in the retinal vessels [16] and choriocapillaries [17] of patients with diabetic retinopathy and in the walls of endoneurial microvessels of patients with diabetic neuropathy [18].

Activation of the complement system may contribute to diabetic vasculopathy via interactions with the coagulation system. Based on the early observation that complement is activated during blood clotting, multiple interactions between the complement and the coagulation/fibrinolysis cascades have been revealed (as reviewed in [19]). Several enzymes of the coagulation/fibrinolysis cascade can activate C3 and, conversely, several components of the complement system appear to be involved in the activation of thrombin and the modulation of platelet aggregation. These observations position the complement system as part of a complex protease network that is characterised by substantial crosstalk between complement and the coagulation/fibrinolysis system. This considerable interplay might represent another cluster of proteins in which complement participates in macro- and microvascular disease risk by conferring procoagulant, hypofibrinolytic and ultimately prothrombotic properties.

In this issue of Diabetologia, Hess and colleagues present novel aspects of the role of C3 in diabetes with regard to CVD risk [20]. They propose a mechanism in which C3 participates in a hypofibrinolytic, and thus prothrombotic, state. It had previously been recognised that C3 is incorporated into fibrin clots, and that this is accompanied by a modified fibre architecture, rendering clots more resistant to lysis [21, 22]. Hess et al. [20] expand on these previous findings by showing that clots from type 1 diabetic patients are more resistant to fibrinolysis, which appears to be due to altered clotting and lysis features of diabetic fibrinogen and C3. In a variety of experiments they demonstrate that clots generated from diabetic fibrinogen incorporate more C3, resulting in more consolidated clots. On the other hand, exposure of both diabetic and control fibrinogen to diabetic C3 produces clots more resistant to lysis. Furthermore, in diabetic individuals, clot lysis time was correlated with plasma C3 levels. The authors concluded that the effect of $\mathrm{C} 3$ on clot properties is more pronounced in diabetes. It was also suggested that systemic C3 levels and C3-mediated fibrin clot formation and lysis properties may be influenced via glycaemic control, although these were essentially uncontrolled observations that clearly need corroborating.

The mechanism of diabetes-dependent C3 incorporation into fibrin clots with concomitant effects on clot characteristics adds to our knowledge of the broad aspects of complement functioning in type 1 and, potentially also, type 2 diabetes, and may in due course be extended to related disorders, including obesity and NAFLD. Unfortunately, Hess et al. [20] did not consider C3 activation in their experiments, which hampers full comprehension of the presented data. For a better understanding of the C3 coagulation/fibrinolysis interaction, it is essential to know to what extent $\mathrm{C} 3$ activation is involved. If the mechanism presented by Hess et al. takes place basically without C3 activation, this would represent a completely 
novel, direct mode of C3 operating on CVD risk, whereby C3 would contribute to a hypofibrinolytic/prothrombotic state solely by its presence in clots, thereby modulating clot properties. In such a scenario, it will be essential to elucidate what intrinsic characteristics of diabetic C3 and fibrinogen underlie the observed changes in clot lysis properties. Glycation of C3 may be a plausible possibility [23]. It will also be interesting to see if similar observations with respect to the role of $\mathrm{C} 3$ in hypofibrinolysis can be made in type 2 diabetic individuals. In summary, this brief commentary gives a brief overview of the emerging roles of C3, C3a and C3a-desarg/ASP in adipose tissue homeostasis, insulin resistance and (development of) diabetes and related cardiometabolic diseases. The epidemiological and experimental evidence presented supports a role for these complement factors in metabolic, atherosclerotic/atherothrombotic and microangiopathic processes. It should be noted that an increasing number of additional complement factors are reported to be expressed in adipose tissue in association with insulin resistance (for example, see [24]). Likewise, a range of additional complement components and regulators have also been shown to be involved in the process of atherosclerosis and atherothrombosis [25]. The current study by Hess et al. [20] expands our knowledge of the multifaceted role of the complement system, C3 in particular, in the pathogenesis of diabetes-associated CVD. Further epidemiological, experimental and clinical studies are needed to differentiate between, and determine the relevance of, different aspects of complement function and activation in the complex and interrelated processes that are characteristic of cardiometabolic disorders. 


\section{References}

1. Cianflone K, Xia Z, Chen LY. Critical review of acylation-stimulating protein physiology in humans and rodents. Biochim. Biophys. Acta, 2003. 1609: 127-43.

2. Engstrom G, Hedblad B, Eriksson KF, Janzon L, Lindgarde F. Complement C3 is a risk factor for the development of diabetes: a population-based cohort study. Diabetes, 2005. 54: 570-5.

3. Onat A, Can G, Rezvani R, Cianflone K. Complement C3 and cleavage products in cardiometabolic risk. Clin. Chim. Acta, 2011. 412: 1171-9.

4. Yesilova Z, Ozata M, Oktenli C, Bagci S, Ozcan A, Sanisoglu SY, Uygun A, Yaman H, Karaeren N, Dagalp K. Increased acylation stimulating protein concentrations in nonalcoholic fatty liver disease are associated with insulin resistance. Am. J. Gastroenterol., 2005. 100: 842-9.

5. Jacobs M, van Greevenbroek MM, van der Kallen CJ, Ferreira I, Blaak EE, Feskens EJ, Jansen EH, Schalkwijk CG, Stehouwer CD. The association between the metabolic syndrome and peripheral, but not coronary, artery disease is partly mediated by endothelial dysfunction: the CODAM study. Eur. J. Clin. Invest., 2011. 41: 167-75.

6. Muscari A, Antonelli S, Bianchi G, Cavrini G, Dapporto S, Ligabue A, Ludovico C, Magalotti D, Poggiopollini G, Zoli M. Serum C3 is a stronger inflammatory marker of insulin resistance than C-reactive protein, leukocyte count, and erythrocyte sedimentation rate: comparison study in an elderly population. Diabetes Care, 2007. 30: $2362-8$.

7. Markiewski MM, Lambris JD. The role of complement in inflammatory diseases from behind the scenes into the spotlight. Am. J. Pathol., 2007. 171: 715-27.

8. Mamane Y, Chung Chan C, Lavallee G, Morin N, Xu LJ, Huang J, Gordon R, Thomas W, Lamb J, Schadt EE, Kennedy BP, Mancini JA. The C3a anaphylatoxin receptor is a key mediator of insulin resistance and functions by modulating adipose tissue macrophage infiltration and activation. Diabetes, 2009. 58: 2006-17.

9. Paglialunga S, Fisette A, Yan Y, Deshaies Y, Brouillette JF, Pekna M, Cianflone K. Acylation-stimulating protein deficiency and altered adipose tissue in alternative complement pathway knockout mice. Am. J. Physiol. Endocrinol. Metab., 2008. 294: E521-529.

10. Wen Y, Wang H, MacLaren R, Wu J, Lu H, Cianflone K. Palmitate and oleate induction of acylation stimulating protein resistance in 3T3-L1 adipocytes and preadipocytes. J. Cell. Biochem., 2008. 104: 391-401.

11. Seifert PS, Hugo F, Hansson GK, Bhakdi S. Prelesional complement activation in experimental atherosclerosis. Terminal C5b-9 complement deposition coincides with cholesterol accumulation in the aortic intima of hypercholesterolemic rabbits. Lab. Invest., 1989. 60: 747-54.

12. Ostergaard J, Hansen TK, Thiel S, Flyvbjerg A. Complement activation and diabetic vascular complications. Clin. Chim. Acta, 2005. 361: 10-9.

13. Xiao X, Ma B, Dong B, Zhao P, Tai N, Chen L, Wong FS, Wen L. Cellular and humoral immune responses in the early stages of diabetic nephropathy in NOD mice. J. Autoimmun., 2009. 32: 85-93.

14. Morita Y, Ikeguchi H, Nakamura J, Hotta N, Yuzawa Y, Matsuo S. Complement activation products in the urine from proteinuric patients. J. Am. Soc. Nephrol., 2000. 11: 700-7.

15. Hsu SI, Couser WG. Chronic progression of tubulointerstitial damage in proteinuric renal disease is mediated by complement activation: a therapeutic role for complement inhibitors? J. Am. Soc. Nephrol., 2003. 14: S186-91.

16. Zhang J, Gerhardinger C, Lorenzi M. Early complement activation and decreased levels of glycosylphosphatidylinositol-anchored complement inhibitors in human and experimental diabetic retinopathy. Diabetes, 2002. 51: 3499-504.

17. Gerl VB, Bohl J, Pitz S, Stoffelns B, Pfeiffer N, Bhakdi S. Extensive deposits of complement C3d and C5b-9 in the choriocapillaris of eyes of patients with diabetic retinopathy. Invest. Ophthalmol. Vis. Sci., 2002. 43: 1104-8.

18. Rosoklija GB, Dwork AJ, Younger DS, Karlikaya G, Latov N, Hays AP. Local activation of the complement system in endoneurial microvessels of diabetic neuropathy. Acta Neuropathol, 2000. 99: 55-62.

19. Oikonomopoulou K, Ricklin D, Ward PA, Lambris JD. Interactions between coagulation and complement--their role in inflammation. Semin. Immunopathol., 2012. 34: 151-65.

20. Hess K, Alzahrani SH, Mathai M, Schroeder V, Carter AM, Howell G, Koko T, Strachan MW, Price JF, Smith KA, Grant PJ, Ajjan RA. A novel mechanism for hypofibrinolysis in diabetes: the role of complement C3. Diabetologia, 2012. 55: 1103-13.

21. Shats-Tseytlina EA, Nair CH, Dhall DP. Complement activation: a new participant in the modulation of fibrin gel characteristics and the progression of atherosclerosis? Blood Coagul. Fibrinolysis, 1994. 5: 529-35.

22. Distelmaier K, Adlbrecht C, Jakowitsch J, Winkler S, Dunkler D, Gerner C, Wagner O, Lang IM, Kubicek M. Local complement activation triggers neutrophil recruitment to the site of thrombus formation in acute myocardial infarction. Thromb. Haemost., 2009. 102: 564-72. 
23. Austin GE, Mullins RH, Morin LG. Non-enzymic glycation of individual plasma proteins in normoglycemic and hyperglycemic patients. Clin. Chem., 1987. 33: 2220-4.

24. Moreno-Navarrete JM, Martinez-Barricarte R, Catalan V, Sabater M, Gomez-Ambrosi J, Ortega FJ, Ricart W, Bluher M, Fruhbeck G, Rodriguez de Cordoba S, Fernandez-Real JM. Complement factor H is expressed in adipose tissue in association with insulin resistance. Diabetes, 2010. 59: 200-9.

25. Speidl WS, Kastl SP, Huber K, Wojta J. Complement in atherosclerosis: friend or foe? J. Thromb. Haemost., 2011. 9: 428-40. 


\section{Chapter 4 Cugibfr +}

Distinct associations of complement C3a and its precursor C3 with atherosclerosis and cardiovascular disease: The CODAM study

Thrombosis and Haemostasis 2014. 111: 1102-11

E. Hertle, M.M.J. van Greevenbroek, I.C.W. Arts, C.J.H. van der Kallen, S.L.C. Geijselaers, E.J.M. Feskens, E.H.J.M. Jansen, C.G. Schalkwijk, C.D.A. Stehouwer 


\begin{abstract}
Complement C3 is a novel risk factor for cardiovascular disease (CVD), but the underlying mechanism is currently unknown. We determined the associations of the anaphylatoxin C3a, the activation product of $\mathrm{C} 3$, and of $\mathrm{C} 3$ itself with estimates of atherosclerosis and CVD.

We studied associations of C3a and C3 with carotid intima-media thickness (cIMT), ankle-arm blood pressure index (AAIx) and CVD in cross-sectional analyses among 545 participants of the Cohort on Diabetes and Atherosclerosis Maastricht (CODAM) study (61\% men, 59.4 \pm 6.9 years) and examined effect modification by smoking. We conducted linear and logistic regression analyses with adjustments for age, sex, glucose metabolism status, lipids, adiposity, renal function, blood pressure, pack years smoked, physical activity, use of medication and investigated mediation by inflammation.

C3a was independently associated with cIMT ( $\beta=0.032 \mathrm{~mm}$, [95\% Cl: $0.004 ; 0.060])$ and AAlx $(\beta=-0.022$, [-0.043; -0.001$])$, but C3 was not. Effect modification by smoking was only observed for CVD ( $\left.P_{\text {smoking }}{ }^{C} 3 a=0.008, P_{\text {smoking }}{ }^{*}{ }_{C 3}=0.018\right)$, therefore these associations were stratified for smoking behaviour. Both C3a $(O R=2.96,[1.15 ; 7.62])$ and C3 (OR =1.98, [1.21; 3.22]) were independently associated with CVD in heavy smokers. The association of C3 with CVD was independent of C3a. Low-grade inflammation did partially explain the association of C3a with AAIx, but not the other observed associations.

This suggests that C3a and C3 have distinct roles in pathways leading to CVD. C3a may promote atherosclerosis and additionally advance CVD in heavy smokers. Conversely, C3 may be associated with CVD in heavy smokers via pathways other than atherosclerosis.
\end{abstract}




\section{Introduction}

Cardiovascular disease (CVD) is an inflammatory disease [1]. CVD results from a combination of atherosclerosis, a chronic disease of the vessel wall, and atherothrombosis, which is an acute event of vessel occlusion. Complement-mediated inflammatory responses are increasingly implicated in the development of CVD [2]. The complement network is part of the innate immune system, orchestrates adaptive immunity and inflammatory networks, and also interacts with coagulation and fibrinolysis [3]. Besides its well-known role in host defence, complement is involved in tissue repair and homeostasis [4] and also appears to be crucial in vascular health. Unbalanced complement function is therefore thought to contribute to a broad spectrum of cardiovascular pathologies, including atherosclerosis and atherothrombosis.

The C3 - C3a - C3a receptor (C3aR) axis is an important effector pathway of the complement network. $\mathrm{C} 3$ is the pivotal protein of the complement cascade, and is cleaved upon activation, resulting in the release of the anaphylatoxin C3a. At present, several cohort studies have shown an association of C3 with metabolic disturbances such as adiposity, dyslipidaemia, insulin resistance and liver dysfunction, as well as with incident diabetes and CVD $[5,6]$. We previously found a similar association between $\mathrm{C} 3$ and coronary heart disease (CHD) in a comparable population, but showed that this association is restricted to smokers [7]. This is in line with studies suggesting that smoking might affect the C3-C3a-C3aR axis [8-11]. It has generally been assumed that these adverse associations of C3 are not due to C3 itself, but to activation of $\mathrm{C} 3$ with generation of $\mathrm{C} 3 \mathrm{a}$. C3a is a proinflammatory mediator that can signal via its specific membrane-bound receptor ( $C 3 a R)$ present on immune cells, endothelial cells and smooth muscle cells [12]. Effects of C3a include release of proinflammatory cytokines, degranulation of mast cells, increase in vascular permeability, smooth muscle cell contraction and chemotaxis of immune cells. However in humans, data on the relation between C3a and the development of CVD are scarce. To the best of our knowledge, only one study has been published on this subject [13]. This case-control study reported elevated plasma C3a concentrations in patients with coronary artery disease (CAD) compared to healthy, age-matched controls, but did not consider potential confounding factors, such as obesity, diabetes, smoking status or use of medication.

To obtain more insight in the role of the C3-C3a axis in the development of human CVD, we investigated the associations of C3 and C3a with several measures of atherosclerosis (carotid intima-media thickness [CIMT] and ankle-arm blood pressure index [AAIx]) and prevalent CVD in a cohort of middle-aged to older Caucasian individuals with a moderately increased risk of type 2 diabetes (T2DM) and CVD. Next, we explored the interaction of C3 and C3a with smoking behaviour, and we evaluated to what extent C3a could explain any associations observed for C3. Finally, we evaluated to what extent low-grade inflammation could explain the associations observed for C3a and C3. 


\section{Materials and Methods}

\subsection{Participants}

The current study presents cross-sectional analyses among 545 participants of the Cohort study on Diabetes and Atherosclerosis Maastricht (CODAM) cohort ( $n=574)$, which has been described in detail previously [14]. Briefly, participants of a large population-based cohort were invited for screening according to the following criteria: Caucasian descent, age $>40$ years, and additionally at least one of the following criteria: body mass index (BMI) $>25$ $\mathrm{kg} / \mathrm{m}^{2}$; use of anti-hypertensive medication; positive family history of T2DM; postprandial blood glucose $>6.0 \mathrm{mmol} / \mathrm{L}$; history of gestational diabetes and/or glucosuria. After an oral glucose tolerance test (OGTT), all those screened with T2DM and a random selection of those with normal glucose metabolism (NGM) and impaired glucose metabolism (IGM, combining impaired fasting glucose and impaired glucose tolerance) were invited to participate in CODAM. According to this inclusion procedure, the CODAM cohort was oversampled for IGM and T2DM. The study was approved by the Medical Ethics Committee of the Maastricht University Medical Centre, and all participants gave written informed consent.

We excluded in all analyses individuals with missing data in C3 or C3a ( $=6$ ) or covariates $(n=23)$. Individuals lacking data on cIMT $(n=41)$ were also excluded in analyses with cIMT as outcome, while in analyses with AAlx as outcome we excluded individuals lacking data on the AAIx $(n=1)$ and those with an AAlx $\geq 1.5(n=3)$, as elevated AAlx values have been linked with vascular stiffness [15]. Thus, we investigated associations with CVD in 545 individuals, associations with cIMT in 504 individuals and associations with AAlx in 541 individuals.

\subsection{Measurements}

Participants were requested to stop lipid-modifying medication 14 days beforehand, while all other medication was stopped the day before the visit. Current smokers were instructed to refrain from smoking from the evening before the blood draw. Blood samples were collected after an overnight fast by venepuncture. Serum was allowed to clot at room temperature for 45 minutes, centrifuged at $1950 \mathrm{~g}$ for 15 minutes and aliquots were stored at $-80^{\circ} \mathrm{C}$ until use. EDTA tubes were kept on ice until blood was centrifuged at $4^{\circ} \mathrm{C}$ (within 3 hours). Plasma aliquots were stored at $-80^{\circ} \mathrm{C}$ until use. Complement $\mathrm{C} 3$ was measured in serum by auto analyser (Hitachi 912) using a Roche kit assay (Roche Diagnostics Nederland BV, Almere, The Netherlands). C3a was determined in EDTA plasma by ELISA (MicroVue C3a plus EIA kit, Quidel, San Diego, USA). This assay measures both the short-lived anaphylatoxin C3a and its stable degradation product C3a-desarg. Inter-assay variation of C3 and C3a were $2.1 \%$ and $6.6 \%$, respectively. The main dependent variables were measured as previously described [16-18]. In short, cIMT was measured in the left and right common carotid artery with an ultrasound scanner (Ultramark $4+$ ) that was placed $10-20 \mathrm{~mm}$ proximal to the carotic bulb. AAlx was measured using a handheld 8-MHZ Doppler device and calculated by dividing the highest of the systolic pressures in the posterior tibial or dorsalis pedis arteries by the highest systolic pressure at the brachial arteries. CVD was defined as the occurrence of at least one of the following: previous myocardial infarction (MI); coronary bypass; 
percutaneous coronary intervention ( $\mathrm{PCl}$ ) or stroke reported by questionnaires (Subjects' Disease History Questionnaire); signs on a 12-lead electrocardiogram of myocardial infarction (Minnesota codes 1-1 or 1-2) or ischaemia (Minnesota codes 1-3, 4-1, 4-2, 4-3, 5 1, 5-2, 5-3 or 7-1); non-traumatic amputation; and/or an AAlx $<0.9$.

Glucose metabolism status was assessed by a standard $75 \mathrm{~g}$ OGTT and classified as NGM, IGM or T2DM according to the WHO criteria [19]. Waist, BMI and blood pressure were measured by a research assistant as described previously [20]. Smoking behaviour was measured in pack years (one pack year is defined as $20 \mathrm{~g}$ of tobacco smoked per day, over the course of one year), reported by questionnaire. Habitual physical activity was assessed by use of a validated short questionnaire [21] and participants were defined as physically active if they were moderately physically active for at least 30 minutes a day for at least 5 days a week [21, 22]. The estimated glomerular filtration rate (eGFR) was calculated with the MDRD [23] formula from creatinine levels, measured in EDTA plasma with a Jaffé diagnostic test (Roche Diagnostics). Other plasma and serum components were measured as described previously: in NaF/KOx plasma: glucose [20]; in EDTA plasma: high density lipoprotein (HDL) cholesterol, total cholesterol, triacylglycerols [20]; in serum: ceruloplasmin and haptoglobin [18]. Interleukin-8 (II-8) and tumour necrosis factor- $\alpha$ (TNF $\alpha$ ) were measured in EDTA plasma using a multiarray detection system (MesoScale Discovery, SECTOR Imager 2400, Gaithersburg, Maryland, USA). C-reactive protein (CRP), Interleukin-6 (II-6), soluble intercellular adhesion molecule-1 (sICAM-1) and serum amyloid A (SAA) were measured twice, once in serum or EDTA plasma with ELISA [24] and once in EDTA plasma using a multiarray detection system (MesoScale Discovery, SECTOR Imager 2400), and were calibrated after cross-validation as described in [25].

\subsection{Statistical analyses}

Variables were visually inspected for normal distribution. Normally distributed variables are presented as meantstandard deviation (SD). Skewed variables are presented as medians with interquartile range (IQR). Skewed variables were $\log _{2}$ transformed to achieve normal distribution prior to the analyses. One-way ANOVA was used to compare continuous or $\log 2$ transformed variables, and Pearson $\mathrm{Chi}^{2}$ to compare categorical variables across tertiles of C3a concentrations. In all regression analyses, the effect sizes for C3a correspond to a doubling in C3a while for C3 they are presented per SD increase in C3.

Main analyses: Multiple linear regression analysis was used to investigate the associations of $\mathrm{C} 3$ and $\log _{2}$ transformed C3a with cIMT and AAlx. Multiple logistic regression analysis was used to investigate the association of C3 and $\log _{2}$ transformed C3a with CVD. We first analysed models that were adjusted for variables inherent to the study design, including stratified sampling for age and sex, and oversampling for IGM and T2DM (model 1: age, sex, glucose metabolism status). Subsequently, we additionally adjusted for blood lipids (model 2: model 1 + triacylglycerols, total cholesterol, HDL-cholesterol) and for weight, fat distribution, systolic blood pressure (SBP), kidney function, pack years and physical activity (model 3: + BMI, waist, SBP, eGFR, smoking, physical activity), because these are known determinants of atherosclerosis and have been associated with C3 and/or C3a [5]. In the full 
model, we further adjusted for use of relevant medication (model 4: + use of glucoselowering medication, use of anti-hypertensive medication) because these are associated with CVD and might influence C3 or C3a levels. All assumptions for linear and logistic regression analyses were met.

Interaction with smoking behaviour: Since we previously observed effect modification by smoking in the association of C3 with CHD [7], we also investigated this interaction in the current analyses. We used the same classification for smoking behaviour, creating one group that combines the never and light smokers (never smokers combined with former and current smokers who smoked $\leq$ median of pack years of all smokers) and a group of heavy smokers (former and current smokers; >median of pack years of all smokers). The median of pack years in ever smokers was 23.0 pack years. We tested for interaction by adding crossproducts of $\mathrm{C} 3 \mathrm{a}$ and smoking behaviour, and of $\mathrm{C} 3$ and smoking behaviour to the respective regression models.

Evaluating the C3-C3a axis: In all fully adjusted models we first performed mutual adjustments for C3 or C3a in order to evaluate their independent associations. Subsequently, we conducted mediation analyses as described elsewhere [26], to determine the extent to which the association(s) of C3, if present, were mediated (i.e. explained) by C3a. In short, we estimated the indirect effect of C3 via C3a on the outcome and computed bootstrapped (bias-corrected) confidence intervals (5000 samples) for the size of the mediated effect, using SPSS macros provided by Preacher and Hayes [27].

Mediation by low-grade inflammation: As C3a is a potent inducer of inflammation and as C3 is also an acute-phase protein, we evaluated the extent to which low-grade inflammation accounted for the observed associations. For this, a low-grade inflammation score, representing an individual's overall low grade inflammatory status, was calculated for each subject by averaging the Z-scores of the 8 markers of low-grade inflammation CRP, IL-6, TNF $\alpha$, IL-8, sICAM-1, SAA, ceruloplasmin and haptoglobin [28]. Mediation analyses were conducted as described above.

Additional analyses: Our a priori CVD definition may result in a heterogeneous group of cases, including patients with atherothrombotic events as well as other patients with CVD. As these cardiovascular pathologies differ in their underlying aetiologies, we repeated the logistic regression analyses after sub-classifying CVD into atherothrombotic events (comprising $\mathrm{MI}$, stroke and/or non-traumatic amputation) and CVD of other aetiology (ischaemia, angina pectoris, coronary bypass, $\mathrm{PCl}$ ). In multinomial logistic regression, both sub-groups of cases were compared to non-CVD subjects (as reference group). Furthermore, we performed several sensitivity analyses. First, we repeated all analyses with exclusion of participants with a possible acute inflammation (serum CRP concentrations $>10 \mathrm{mg} / \mathrm{L}, \mathrm{n}=37$ ). In the associations with AAlx, we additionally repeated the analyses with exclusion of participants with clinical peripheral arterial disease ( $P A D, n=29)$ in order to preclude influential impact of PAD patients; and also with exclusion of participants with an AAlx higher than $1.3(n=18)$, as occasionally 1.3 is proposed as cut-off for vascular stiffness [15]. Furthermore, we performed additional adjustments for use of lipid-modifying medication [yes/no], which might in crosssectional evaluations act both as a confounder and as a collider. A collider is defined as a 
common effect of exposure (or of causes of exposure) and outcome [29]. Particularly in the analyses with CVD as outcome, lipid-modifying medication might be a collider, because lipidmodifying medication is in practice often prescribed after a clinical event, regardless of actual lipid profiles, and can therefore be considered a consequence of disease. On the other hand, lipid-modifying medication might act as a confounder when this is a determinant of CVD, and might also influence C3 or C3a levels [30]. For as far as lipid-modifying medication acts as a confounder, failure to adjust for it may result in residual confounding, while adjustment may generate bias if it acts as a collider [29]. Finally, we investigated effect modification by sex by adding interaction terms to the fully adjusted models. We found no such interaction ( $P$ for interaction $>0.1$ ) and therefore all results are presented for men and women combined.

All statistical analyses were performed using the SPSS package version 18.0 (SPSS, Chicago, IL, USA) and statistical significance was set at $P<0.05$, except for testing for interaction where statistical significance was set at $P<0.10$.

\section{Results}

\subsection{General characteristics of the study population}

C3a levels ranged from 26 to $212 \mathrm{mg} / \mathrm{L}$, with a median of 59 [IQR: 49-73] mg/L. C3 levels ranged from 1.01 to $3.35 \mathrm{~g} / \mathrm{L}$, with a mean of $1.80 \pm 0.33 \mathrm{~g} / \mathrm{L}$. C3a and C3 concentrations were modestly correlated (Spearman's correlation coefficient 0.34, $\mathrm{P}<0.001$ ). General characteristics of the study population across tertiles of C3a concentration and tertiles of C3 concentration are presented in table 1. Participants in the higher tertiles of plasma C3a had higher plasma C3, BMI, waist circumference, systolic blood pressure, HOMA2IR, CRP, IL-6 and TNF $\alpha$ compared to participants in the lowest C3a tertile. Furthermore, the higher tertiles of C3a were characterized by a greater proportion of women and a greater proportion of participants using lipid-modifying and anti-hypertensive medication than the lowest tertile. Compared to the lowest tertile, individuals in the higher tertiles of C3a concentration also more often had CVD, CHD and PAD. Notably, only HDL-cholesterol but not triacylglycerides, LDL-cholesterol or total cholesterol differed among tertiles of C3a.

\subsection{Associations of C3a and C3 with markers of atherosclerosis and CVD}

Plasma C3a was positively associated with cIMT and inversely with the AAIx (table 2, model $1 \mathrm{a}$, adjusted for age, sex and glucose metabolism status). A doubling in plasma C3a was associated with $0.043 \mathrm{~mm}$ higher $\mathrm{cIMT}$ [95\% $\mathrm{Cl}$ : 0.015; 0.071] and with 0.030 lower AAlx [95\% Cl: $-0.051 ;-0.009]$, both reflecting more atherosclerosis. A doubling in C3a represents the range between the median of the 1st and the 3rd tertile of C3a (see table 1 ). When model 1a was additionally adjusted for lipids (model 2a) the associations were only marginally attenuated. The associations of C3a with CIMT and AAIx also remained significant when all potential confounders were included (model 4a). In this fully adjusted model, a doubling in plasma C3a was associated with $0.032 \mathrm{~mm}$ higher CIMT [95\% Cl: 0.004; 0.060] and with 0.022 lower AAlx [95\%Cl: -0.043; -0.001]. Plasma C3a was also positively associated with prevalent 


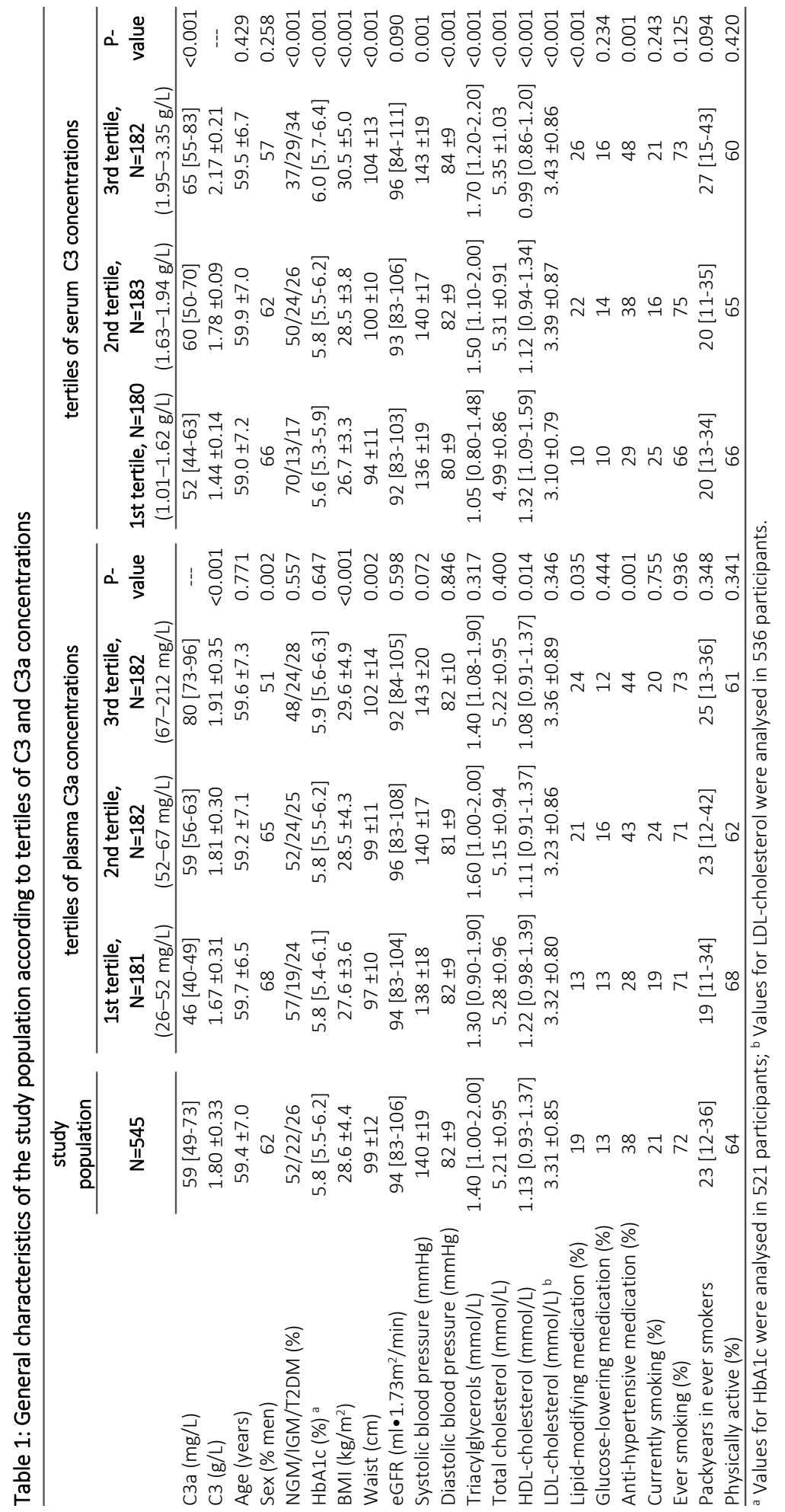




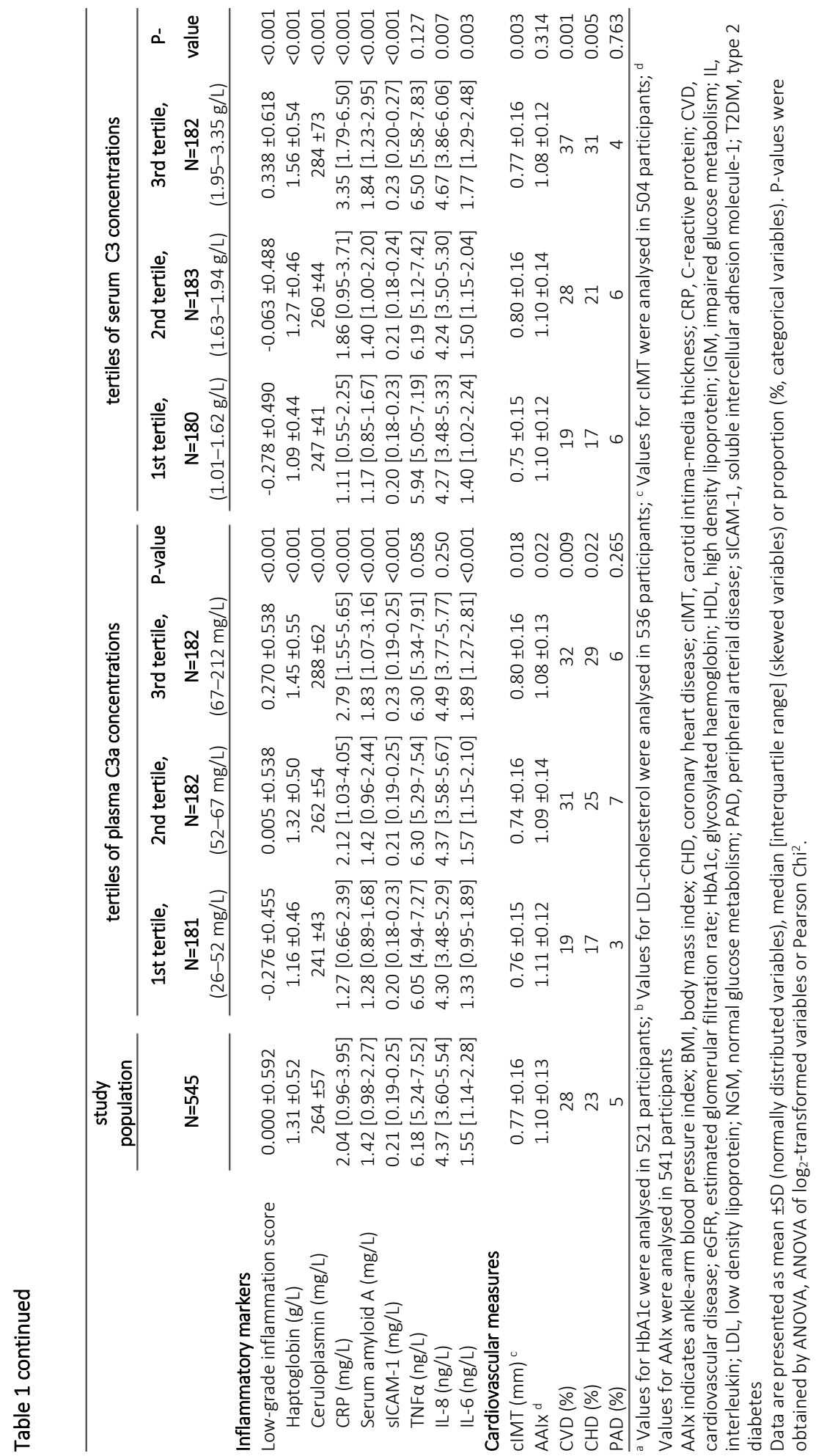


CVD (table 3, all participants, model 1a), but this association was substantially attenuated and became non-significant in the fully adjusted model (model 4a).

C3 was not associated with the AAlx in any of the linear regression analyses (table 2, models 1b-4b). C3 was positively associated with cIMT in the minimally adjusted model (table 2, model $1 \mathrm{~b}$ ) but in the fully adjusted model (model $4 \mathrm{~b}$ ) the association with cIMT was almost completely lost. In contrast, C3 was associated with CVD (table 3, all participants, models 1b$4 b)$. In the fully adjusted model, the odds ratio (OR) was 1.42 [95\% Cl: 1.10; 1.84].

\subsection{Effect modification by smoking behaviour}

Addition of cross-products of smoking behaviour and plasma C3a or C3 to the respective linear regression models indicated that smoking behaviour did not modify the associations of C3a or C3 with CIMT (Psmoking*C3a $=0.712$; $P_{\text {smoking*C3 }}{ }^{*}=0.730$ ) or with the AAIX ( $P_{\text {smoking }}{ }^{C}{ }_{3 a}=0.482 ; \quad P_{\text {smoking }}{ }^{C} 3=0.243$ ). In contrast, we observed significant interaction between smoking and C3a with regard to the association between C3a and CVD ( $P_{\text {smoking }}{ }^{*}$ C3a $=0.008$ ). In line with our previous report on C3 and CHD [12], the association of C3 with CVD also differed according to smoking behaviour ( $\left.P_{\text {smoking }}{ }^{*} 3=0.018\right)$. Subsequent stratified analyses revealed that C3a was independently and positively associated with CVD in heavy smokers but not in never/light smokers (table 3). In heavy smokers, the OR was 4.06 [95\% Cl: $1.71 ; 9.61$ ] (model 1a). This association remained significant when all potential confounders were included (model 4a, OR was 2.96 [95\% Cl: 1.15; 7.62]). Likewise, C3 was independently and positively associated with CVD in the fully adjusted model, again only in heavy smokers (table 3, model 4b, OR was 1.98 [95\% Cl: 1.21; 3.22]).

\subsection{Mutual independence of the associations of C3 and C3a}

When we included C3 and C3a simultaneously in the fully adjusted models in table 2 and 3 respectively, adjustment for C3 slightly strengthened the observed associations of C3a with CIMT and AAIx (table 2, model 5a). The associations of C3 and C3a with CVD were only partly attenuated after mutual adjustments (table 3, models $5 \mathrm{a}$ and $5 \mathrm{~b}$ ). In heavy smokers, the association of C3 with CVD remained significant after adjustment for C3a ( $P=0.014)$, while the association of C3a with CVD was attenuated and became non-significant after adjustment for C3 ( $P=0.059)$. Also, in a formal mediation model, the association of C3 in heavy smokers was not explained by C3a (see supplemental figure 1), as the effect of C3 on CVD mediated via C3a was small and not significant (indirect effect of C3 via C3a: OR was 1.09, 95\% Cl: 0.94; 1.26).

\subsection{The role of low-grade inflammation in the associations of C3a and C3}

We performed mediation analyses to determine the extent to which low-grade inflammation accounted for the significant associations observed for C3a and C3 in paragraph 3.2 and 3.3. Low-grade inflammation explained $\approx 30 \%$ of the association of C3a with AAlx (indirect effect of C3a via low-grade inflammation: $\beta$ was $-0.007,95 \% \mathrm{Cl}$ : -0.016 ; -0.001 ). In contrast, lowgrade inflammation did not significantly attenuate the associations of C3a with cIMT, and of C3a or C3 with CVD in the group of heavy smokers (see supplemental figures 2a-d). 


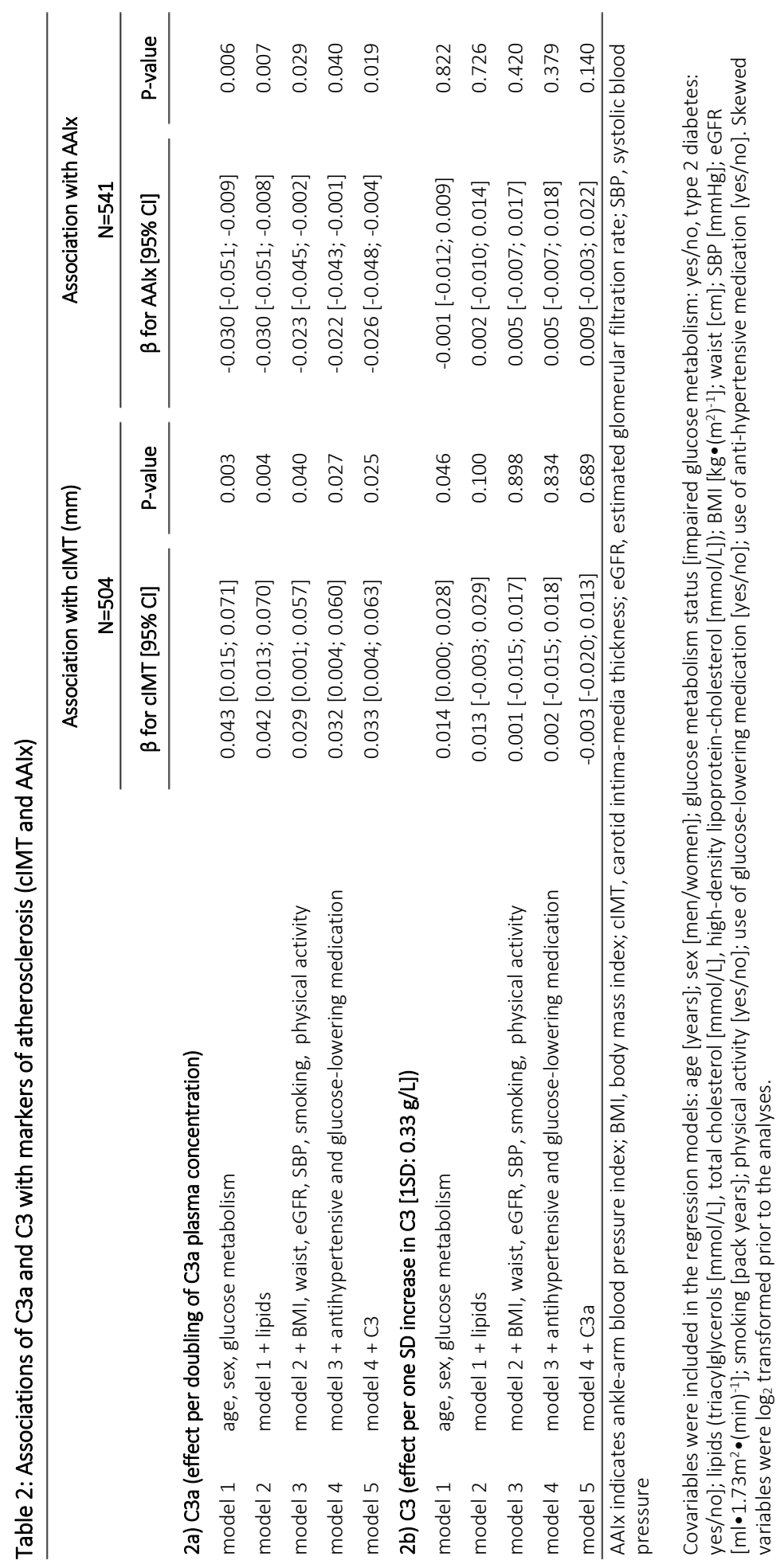




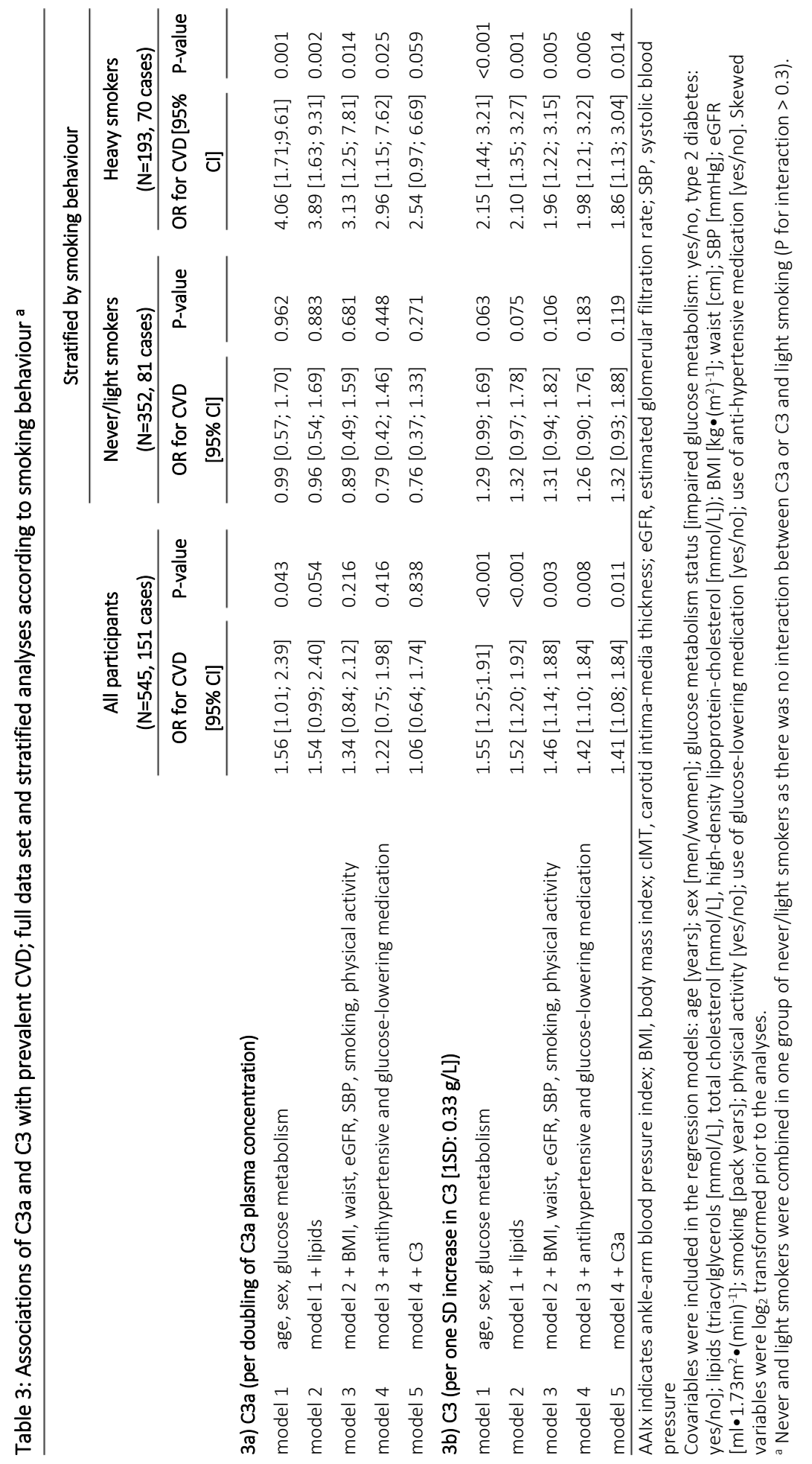




\subsection{Additional analyses}

To explore the observed differences in the associations of C3a and C3 with markers of atherosclerosis and CVD, we sub-classified the outcome measure in the logistic regression analyses into atherothrombotic events ( $N=35$ in the heavy smokers) and into events of other aetiology ( $\mathrm{N}=35$ in the heavy smokers) In this sub-analysis, the association of C3 with atherothrombotic events was stronger than the association with overall CVD (70 cases in the heavy smokers, see table 3, model $4 \mathrm{~b}$ ), while there was no association of C3 with nonatherothrombotic events: in the fully adjusted model, the OR for atherothrombotic events in the group of heavy smokers was $3.04[95 \% \mathrm{Cl}: 1.55 ; 5.96, P=0.001]$ and the OR for nonatherothrombotic events was 1.34 [0.74; 2.43, P=0.336]. Upon additional adjustment for C3a, the OR for atherothrombotic events was unchanged [OR was 2.88, 95\% Cl: $1.47 ; 5.67$, $\mathrm{P}=0.002]$, indicating that this association of C3 was fully independent of C3a. On the other hand, C3a was in this sub-analysis equally associated with atherothrombotic events (OR was 2.92, 95\% $\mathrm{Cl}: 0.84 ; 10.18, \mathrm{P}=0.092$ ) as with non-atherothrombotic events (OR was 2.92, 95\% Cl: 0.91; 9.32, $\mathrm{P}=0.071$ ). Additional adjustments for C3 did not materially alter these results (data not shown).

In the sensitivity analyses, exclusion of participants with serum CRP concentrations above 10 $\mathrm{mg} / \mathrm{L}$, or of participants with PAD or AAIx $>1.3$ did not materially alter the results (data not shown). Additional adjustment for use of lipid-modifying medication in the fully adjusted models did not materially alter the associations of C3a with cIMT ( $\beta$ was $0.031,95 \% \mathrm{Cl}: 0.002$; $0.059, \mathrm{P}=0.034$ ) and AAlx ( $\beta$ was $-0.021,95 \% \mathrm{Cl}:-0.043 ; 0.000, \mathrm{P}=0.051$ ). In contrast, in the models with CVD as outcome additional adjustment for use of lipid-modifying medication in the fully adjusted model did slightly attenuate the association of C3a (heavy smokers: OR was 2.47, 95\% Cl: $0.88 ; 6.92, \mathrm{P}=0.084$ ) and of $\mathrm{C} 3$ (heavy smokers: OR was $1.79 ; 95 \% \mathrm{Cl}: 1.00 ; 3.02$, $\mathrm{P}=0.031)$.

\section{Discussion}

The current study has four main findings. First, higher concentrations of C3a, but not of C3, were independently associated with more severe atherosclerosis, as represented by higher CIMT and lower AAlx. Second, we observed strong and significant effect modification by smoking behaviour in the associations of both C3 and C3a with CVD. Only in the group of heavy smokers were C3 and C3a associated with CVD, again independently of a large number of confounders. Sub-analyses showed that in heavy smokers, C3 was specifically associated with atherothrombotic events, while C3a was equally associated with atherothrombotic events and events of other aetiology. Third, the associations of C3 with CVD and atherothrombotic events were independent of C3a. Finally, systemic low-grade inflammation explained $\approx 30 \%$ of the association between C 3 a and AAlx, but did not significantly attenuate the association of C3a with CIMT or of C3a and C3 with CVD in heavy smokers.

This is the first large cohort study that has investigated the C3-C3a axis in human CVD. To the best of our knowledge, there is only one former study on C3a in human CVD [13], which showed that C3a was higher in patients with CAD than in healthy, age-matched controls. 
Furthermore, $\mathrm{C} 3 \mathrm{a}$ has been shown to be higher in unstable compared to stable angina pectoris [31]. Other studies have focused on complications of established CVD and found C3a a prognostic marker in patients with heart failure [32] or receiving a stent implantation [33]. However, these previous studies either lacked important potential confounders, were small or restricted, and C3 was not measured.

Our current data clearly show that C3 and C3a may reflect different pathways contributing to CVD. C3a was independently associated with cIMT and AAIx, but C3 was not. This lack of an association of $\mathrm{C} 3$ with estimates of atherosclerosis is remarkable, given the existing body of evidence that has implicated C3 in CVD. As far as we are aware, our study is the first on the association of $\mathrm{C} 3$ with AAlx. Previous studies on the relation of $\mathrm{C} 3$ with $\mathrm{CIMT}$ were inconclusive, [34-36]. Notably, the association of C3 with CVD in heavy smokers was only partly attenuated by adding C3a to the model and was independent of C3a. Also, in a mediation model C3a did not account for the association of C3 with CVD. This suggests that C3a does not explain a substantial part of the cardiovascular pathology associated with circulating C3. Despite the fact that positive associations of C3 with adverse cardiovascular outcomes $[5,6]$ have often been attributed to C3 activation with generation of C3a, this assumption is not supported by our current observations.

The discrepancies between C3a and C3 in their associations with IMT, AAIx and CVD might be explained by the concept that atherosclerosis and CVD reflect different aspects of the aetiology of vascular disease. In our current analyses, cIMT and AAIx predominantly reflect atherosclerosis, whereas CVD may additionally involve atherothrombosis, and possibly also hypofibrinolysis [37]. Thus, C3a may be involved in atherogenesis, while C3 seems to be a determinant of atherothrombosis, apparently via mechanisms influenced by smoking. To evaluate this concept we reanalysed the data by sub-classifying CVD into atherothrombotic and non-atherothrombotic CVD. In these analyses, C3 was exclusively associated with atherothrombosis, and this association was stronger than with overall CVD. In contrast, C3a was equally associated with both subclasses of CVD.

The observed associations of C3a with atherosclerosis are in agreement with the well-known proinflammatory and proposed proatherogenic effects of C3a. C3a-receptors are present on immune cells, smooth muscle cells and endothelial cells, and were also detected in human atherosclerotic plaques [38]. Complement activation and generation of C3a might promote atherosclerosis via recruitment and activation of immune cells and via stimulation of smooth muscle cell proliferation, endothelial activation and induction of low-grade inflammation [39, 40]. When we explored the role of low-grade inflammation in the associations observed for C3a, we found that a significant portion $(\approx 30 \%)$ of the association of C3a with AAlx, but not with cIMT or CVD, was explained by low-grade inflammation. This suggests that C3a might partly contribute to cardiovascular dysfunction via induction of low-grade inflammation, but other routes may be relevant as well. Notably, the associations of C3a and C3 with CVD in the heavy smokers (discussed in detail below) were not significantly explained by low-grade inflammation. We stress that our approach of averaging 8 biomarkers of low-grade inflammation produces a robust estimate of the overall inflammatory state with 
minimization of random error. This suggests that C3a and C3 may affect the underlying processes - at least partly - via other routes than inflammation.

Interestingly, our current analyses showed that smoking behaviour modified the association of C3a with CVD. An interaction of C3a with smoking behaviour is supported by observations showing that cigarette smoke enhanced C3aR mRNA expression in vascular myofibroblasts [10], and that smoking was shown to activate the transcription factor activator-protein-1 [41], which regulates C3aR expression [42]. Accordingly, smoking might render an individual hypersensitive for $\mathrm{C} 3 \mathrm{a}$, but we do not have a clear explanation of how smoking could affect the impact of C3a on CVD, but not on atherosclerosis. Taken together, our findings suggest that C3a might in heavy smokers promote CVD of both atherothrombotic and nonatherothrombotic aetiology, but the underlying mechanisms remain to be established.

In our previous publication on the association of C3 with CHD [7] we had observed that this association was actually attributable to the group of former and current heavy smokers, even though the association was also detectable in the whole CODAM population. We proposed that this interaction might originate from higher C3 activation and C3a generation in heavy smokers [7]. Accordingly, one would expect higher C3a in heavy smokers, but in the current study C3a concentrations were not higher in the heavy (median C3a=61 ng/ml) than in never/light smokers (median C $3 a=58 \mathrm{ng} / \mathrm{ml}$, non-significant). This observation actually points to a discrete interaction of smoking and C3 that is not mediated via effects of smoking on C3a generation. Interestingly, C3 may also be non-proteolytically activated to form the reactive intermediate $\mathrm{C} 3 \bullet \mathrm{H} 2 \mathrm{O}$ without release of $\mathrm{C} 3 \mathrm{a}$ [43]. Non-proteolytic complement activation may occur on activated platelets $[43,44]$ and might also underlie smoking-induced C3 activation [9]. Furthermore, C3 is a structural component of fibrin clots and was recently implicated in hypofibrinolysis $[45,46]$, although the precise molecular mechanism is as yet unidentified. In our view, both these processes might, at least to some extent, explain the observed associations of C3 with atherothrombosis rather than atherosclerosis that we presented herein.

With respect to the associations observed for $\mathrm{C} 3 \mathrm{a}$ in the present evaluations, one should keep in mind that the assay we used to determine the plasma C3a concentration measures both C3a and its stable degradation product C3a-desarg. C3a-desarg was described as a lipogenic hormone with crucial functions in plasma lipid metabolism [47], but its role in atherosclerosis is as yet unclear. In our human cohort, C3a/C3a-desarg was not associated with triacylglycerols, total or LDL-cholesterol (table 1), and adjustment for plasma lipids did not affect the associations with CIMT, AAIx or CVD (regression models 2a versus 1a). Thus, the beneficial effects of C3a-desarg on lipid metabolism appear to be less prominent in human atherosclerosis. However, we measured C3a/C3a-desarg in the fasted state, which may incompletely reflect its function in postprandial lipaemia [48, 49]. Murine data also support the relevance of $\mathrm{C} 3 \mathrm{a}$ in atherosclerosis, as transgenic mice lacking the C3aR, a receptor specific for $\mathrm{C} 3 \mathrm{a}$ but not activated by $\mathrm{C} 3 \mathrm{a}$-desarg, had less atherosclerosis than their respective control $[50,51]$.

The most prominent strengths of our study are the evaluation of both C3 and C3a in relation to several aetiological measures of CVD within one cohort, and the comprehensive 
characterization for potential confounding factors. Our study does, however, also have limitations. First, the cross-sectional design precludes any conclusion on the temporal relationships between exposure(s) and outcome. Nevertheless, it is implausible that the observed associations of C3 and C3a are due to reverse causation, as complement is one of the most upstream sensor and effector systems for downstream inflammatory cycles. In addition, C3 predicted cardiovascular outcomes in several prospective cohorts [5], and a large body of experimental data indicates an active contribution of $\mathrm{C} 3 \mathrm{a}$ in inflammatory processes [52]. In addition, our observations on the distinct associations of C3a and C3 with atherothrombotic events and events of other aetiology were obtained in post-hoc subanalyses and await replication in other cohorts. Also the use of a selected study population might limit the generalizability of our results. Notwithstanding, our study population does reflect the large group of elderly individuals that are (centrally) overweight, modestly hypertensive, have some signs of a disturbed glucose metabolism and/or use medication for various reasons. Lastly, we measured systemic concentrations of C3 and $\mathrm{C} 3 \mathrm{a}$ as reflections of pathological processes, but activation of the C3-C3a axis may mainly take place locally in the vasculature. Systemic C3 may be a good reflection of local C3 availability, while systemic C3a may be a less efficient reflection of locally generated C3a. This limitation would, however, result in an underestimation of any effects of C3a and hence even emphasizes its relevance in the presented associations.

In conclusion, our findings strongly suggest that C3 and C3a reflect different pathways in CVD. We found that C3a was independently associated with markers of atherosclerosis in the whole cohort, while it was associated with CVD only in heavy smokers. In contrast, C3 was not associated with markers of atherosclerosis. Thus, while C3 was, in heavy smokers, also associated with CVD, this was via pathways other than atherosclerosis. Moreover, this latter association was not mediated by C3a. These distinct associations with atherosclerosis and CVD might be explained by the concept that CVD comprises not only atherosclerosis but also atherothrombosis and possibly hypofibrinolysis. Thus, C3a may particularly be involved in the process of atherosclerosis and, in heavy smokers, additionally advance CVD, whereas C3 may affect the process of atherothrombosis, but only in heavy smokers. Our findings suggest that the CVD risk of C3 cannot be explained by an association with atherosclerosis or by its activation marker C3a. C3 and C3a might thus reflect discrete, non-overlapping processes contributing to CVD. 


\section{References}

1. Libby P. Inflammation and cardiovascular disease mechanisms. Am. J. Clin. Nutr., 2006. 83: 456S-460S.

2. Szeplaki G, Varga L, Fust G, Prohaszka Z. Role of complement in the pathomechanism of atherosclerotic vascular diseases. Mol. Immunol., 2009. 46: 2784-93.

3. Oikonomopoulou K, Ricklin D, Ward PA, Lambris JD. Interactions between coagulation and complement--their role in inflammation. Semin. Immunopathol., 2012. 34: 151-65.

4. Ricklin D, Hajishengallis G, Yang K, Lambris JD. Complement: a key system for immune surveillance and homeostasis. Nat. Immunol., 2010. 11: 785-97.

5. Onat A, Can G, Rezvani R, Cianflone K. Complement C3 and cleavage products in cardiometabolic risk. Clin. Chim. Acta, 2011. 412: 1171-9.

6. Hertle E, van Greevenbroek MM, Stehouwer CD. Complement C3: an emerging risk factor in cardiometabolic disease. Diabetologia, 2012. 55: 881-4.

7. van Greevenbroek MM, Jacobs M, van der Kallen CJ, Blaak EE, Jansen EH, Schalkwijk CG, Feskens EJ, Stehouwer $\mathrm{CD}$. Human plasma complement $\mathrm{C} 3$ is independently associated with coronary heart disease, but only in heavy smokers (the CODAM study). Int. J. Cardiol., 2012. 154: 158-62.

8. Yin W, Ghebrehiwet B, Weksler B, Peerschke El. Regulated complement deposition on the surface of human endothelial cells: effect of tobacco smoke and shear stress. Thromb. Res., 2008. 122: 221-8.

9. Kew RR, Ghebrehiwet B, Janoff A. Characterization of the third component of complement (C3) after activation by cigarette smoke. Clin. Immunol. Immunopathol., 1987. 44: 248-58.

10. Helske S, Oksjoki R, Lindstedt KA, Lommi J, Turto H, Werkkala K, Kupari M, Kovanen PT. Complement system is activated in stenotic aortic valves. Atherosclerosis, 2008. 196: 190-200.

11. Phillips CM, Kesse-Guyot E, Ahluwalia N, McManus R, Hercberg S, Lairon D, Planells R, Roche HM. Dietary fat, abdominal obesity and smoking modulate the relationship between plasma complement component 3 concentrations and metabolic syndrome risk. Atherosclerosis, 2012. 220: 513-9.

12. Klos A, Tenner AJ, Johswich KO, Ager RR, Reis ES, Kohl J. The role of the anaphylatoxins in health and disease. Mol. Immunol., 2009. 46: 2753-66.

13. Cianflone K, Zhang XJ, Genest J, Jr., Sniderman A. Plasma acylation-stimulating protein in coronary artery disease. Arterioscler. Thromb. Vasc. Biol., 1997. 17: 1239-44.

14. Kruijshoop M, Feskens EJ, Blaak EE, de Bruin TW. Validation of capillary glucose measurements to detect glucose intolerance or type 2 diabetes mellitus in the general population. Clin. Chim. Acta, 2004. 341: 33-40.

15. Potier L, Abi Khalil C, Mohammedi K, Roussel R. Use and utility of ankle brachial index in patients with diabetes. Eur. J. Vasc. Endovasc. Surg., 2011. 41: 110-6.

16. Atsma F, Bartelink ML, Grobbee DE, van der Schouw YT. Best reproducibility of the ankle-arm index was calculated using Doppler and dividing highest ankle pressure by highest arm pressure. J. Clin. Epidemiol., 2005. 58: $1282-8$.

17. Engelen L, Ferreira I, Gaens KH, Henry RM, Dekker JM, Nijpels G, Heine RJ, t Hart LM, van Greevenbroek MM, van der Kallen CJ, Blaak EE, Feskens EJ, Ten Cate H, Stehouwer CD, Schalkwijk CG. The association between the -374T/A polymorphism of the receptor for advanced glycation endproducts gene and blood pressure and arterial stiffness is modified by glucose metabolism status: the Hoorn and CoDAM studies. J. Hypertens., 2010. 28: 285-93.

18. Jacobs M, van Greevenbroek MM, van der Kallen CJ, Ferreira I, Blaak EE, Feskens EJ, Jansen EH, Schalkwijk CG, Stehouwer CD. The association between the metabolic syndrome and peripheral, but not coronary, artery disease is partly mediated by endothelial dysfunction: the CODAM study. Eur. J. Clin. Invest., 2011. 41: 16775.

19. Alberti KG, Zimmet PZ. Definition, diagnosis and classification of diabetes mellitus and its complications. Part 1: diagnosis and classification of diabetes mellitus provisional report of a WHO consultation. Diabet. Med., 1998. 15: 539-53.

20. van Greevenbroek MM, Jacobs M, van der Kallen CJ, Vermeulen VM, Jansen EH, Schalkwijk CG, Ferreira I, Feskens EJ, Stehouwer CD. The cross-sectional association between insulin resistance and circulating complement C3 is partly explained by plasma alanine aminotransferase, independent of central obesity and general inflammation (the CODAM study). Eur. J. Clin. Invest., 2011. 41: 372-379.

21. Wendel-Vos GC, Schuit AJ, Saris WH, Kromhout D. Reproducibility and relative validity of the short questionnaire to assess health-enhancing physical activity. J. Clin. Epidemiol., 2003. 56: 1163-9.

22. Kemper H, Ooijendijk W, Stiggelbout M. Consensus over de Nederlandse Norm voor Gezond Bewegen. Tijdschr. Soc. Gezondheidsz., 2000. 78: 180-183.

23. Levey AS, Bosch JP, Lewis JB, Greene T, Rogers N, Roth D. A more accurate method to estimate glomerular filtration rate from serum creatinine: a new prediction equation. Modification of Diet in Renal Disease Study Group. Ann. Intern. Med., 1999. 130: 461-70. 
24. Jacobs M, van Greevenbroek MM, van der Kallen CJ, Ferreira I, Blaak EE, Feskens EJ, Jansen EH, Schalkwijk CG, Stehouwer CD. Low-grade inflammation can partly explain the association between the metabolic syndrome and either coronary artery disease or severity of peripheral arterial disease: the CODAM study. Eur. J. Clin. Invest., 2009. 39: 437-44.

25. van Bussel BC, Ferreira I, van de Waarenburg MP, van Greevenbroek MM, van der Kallen CJ, Henry RM, Feskens EJ, Stehouwer CD, Schalkwijk CG. Multiple Inflammatory Biomarker Detection in a Prospective Cohort Study: A Cross-Validation between Well-Established Single-Biomarker Techniques and an Electrochemiluminescense-Based Multi-Array Platform. PLoS ONE, 2013. 8: e58576.

26. Thewissen MM, Damoiseaux JG, Duijvestijn AM, van Greevenbroek MM, van der Kallen CJ, Feskens EJ, Blaak EE, Schalkwijk CG, Stehouwer CD, Cohen Tervaert JW, Ferreira I. Abdominal fat mass is associated with adaptive immune activation: the CODAM Study. Obesity (Silver Spring), 2011. 19: 1690-8.

27. Preacher KJ, Hayes AF. Asymptotic and resampling strategies for assessing and comparing indirect effects in multiple mediator models. Behav. Res. Methods, 2008. 40: 879-91.

28. Wlazlo N, van Greevenbroek MM, Ferreira I, Jansen EJ, Feskens EJ, van der Kallen CJ, Schalkwijk CG, Bravenboer B, Stehouwer CD. Low-grade inflammation and insulin resistance independently explain substantial parts of the association between body fat and serum C3: the CODAM study. Metabolism, 2012. 61: 1787-96.

29. Cole SR, Platt RW, Schisterman EF, Chu H, Westreich D, Richardson D, Poole C. Illustrating bias due to conditioning on a collider. Int. J. Epidemiol., 2010. 39: 417-20.

30. Muscari A, Bastagi L, Poggiopollini G, Tomassetti V, Massarelli G, Boni P, Puddu P. Short term effect of atorvastatin and vitamin $\mathrm{E}$ on serum levels of $\mathrm{C} 3$, a sensitive marker of the risk of myocardial infarction in men. Cardiovasc. Drugs. Ther., 2001. 15: 453-8.

31. Kostner KM, Fahti RB, Case C, Hobson P, Tate J, Marwick TH. Inflammation, complement activation and endothelial function in stable and unstable coronary artery disease. Clin. Chim. Acta, 2006. 365: 129-34.

32. Gombos T, Forhecz Z, Pozsonyi Z, Szeplaki G, Kunde J, Fust G, Janoskuti L, Karadi I, Prohaszka Z. Complement anaphylatoxin C3a as a novel independent prognostic marker in heart failure. Clin. Res. Cardiol., 2012. 101: 607-15.

33. Speidl WS, Katsaros KM, Kastl SP, Zorn G, Huber K, Maurer G, Wojta J, Christ G. Coronary late lumen loss of drug eluting stents is associated with increased serum levels of the complement components C3a and C5a. Atherosclerosis, 2010. 208: 285-9.

34. De Pergola G, Ciccone MM, Guida P, Morea G, Giannuzzo E, Cortese F, Scicchitano P, Favale S, Silvestris F. Relationship between C3 Levels and Common Carotid Intima-Media Thickness in Overweight and Obese Patients. Obes. Facts, 2011. 4: 159-63.

35. Thompson T, Sutton-Tyrrell K, Wildman RP, Kao A, Fitzgerald SG, Shook B, Tracy RP, Kuller LH, Brockwell S, Manzi S. Progression of carotid intima-media thickness and plaque in women with systemic lupus erythematosus. Arthritis Rheum., 2008. 58: 835-42.

36. Rua-Figueroa I, Arencibia-Mireles O, Elvira M, Erausquin C, Ojeda S, Francisco F, Naranjo A, Rodriguez-Gallego C, Garcia-Laorden I, Rodriguez-Perez J, Rodriguez-Lozano C. Factors involved in the progress of preclinical atherosclerosis associated with systemic lupus erythematosus: a 2-year longitudinal study. Ann. Rheum. Dis., 2010. 69: 1136-9.

37. Collet JP, Allali Y, Lesty C, Tanguy ML, Silvain J, Ankri A, Blanchet B, Dumaine R, Gianetti J, Payot L, Weisel JW, Montalescot G. Altered fibrin architecture is associated with hypofibrinolysis and premature coronary atherothrombosis. Arterioscler. Thromb. Vasc. Biol., 2006. 26: 2567-73.

38. Oksjoki R, Laine P, Helske S, Vehmaan-Kreula P, Mayranpaa MI, Gasque P, Kovanen PT, Pentikainen MO. Receptors for the anaphylatoxins C3a and C5a are expressed in human atherosclerotic coronary plaques. Atherosclerosis, 2007. 195: 90-9.

39. Han Y, Fukuda N, Ueno T, Endo M, Ikeda K, Xueli Z, Matsumoto T, Soma M, Matsumoto K. Role of complement $3 a$ in the synthetic phenotype and angiotensin II-production in vascular smooth muscle cells from spontaneously hypertensive rats. Am. J. Hypertens., 2012. 25: 284-9.

40. Monsinjon T, Gasque P, Chan P, Ischenko A, Brady JJ, Fontaine MC. Regulation by complement C3a and C5a anaphylatoxins of cytokine production in human umbilical vein endothelial cells. FASEB J., 2003. 17: $1003-14$.

41. Li YT, He B, Wang YZ. Exposure to cigarette smoke upregulates AP-1 activity and induces TNF-alpha overexpression in mouse lungs. Inhal. Toxicol., 2009. 21: 641-7.

42. Schaefer M, Konrad S, Thalmann J, Rheinheimer C, Johswich K, Sohns B, Klos A. The transcription factors AP1 and Ets are regulators of C3a receptor expression. J. Biol. Chem., 2005. 280: 42113-23.

43. Nilsson B, Nilsson Ekdahl K. The tick-over theory revisited: Is C3 a contact-activated protein? Immunobiology, 2012. 217: 1106-10.

44. Saggu G, Cortes C, Emch HN, Ramirez G, Worth RG, Ferreira VP. Identification of a novel mode of complement activation on stimulated platelets mediated by properdin and C3(H2O). J. Immunol., 2013. 190: 6457-67. 
45. Howes JM, Richardson VR, Smith KA, Schroeder V, Somani R, Shore A, Hess K, Ajjan R, Pease RJ, Keen JN, Standeven KF, Carter AM. Complement C3 is a novel plasma clot component with anti-fibrinolytic properties. Diab. Vasc. Dis. Res., 2012. 9: 216-25.

46. Nikolajsen CL, Scavenius C, Enghild JJ. Human complement C3 is a substrate for transglutaminases. A functional link between non-protease-based members of the coagulation and complement cascades. Biochemistry (Mosc.), 2012. 51: 4735-42.

47. MacLaren R, Cui W, Cianflone K. Adipokines and the immune system: an adipocentric view. Adv. Exp. Med. Biol., 2008. 632: 1-21.

48. Cianflone K, Paglialunga S, Roy C. Intestinally derived lipids: metabolic regulation and consequences--an overview. Atheroscler. Suppl., 2008. 9: 63-8.

49. Cianflone K, Xia Z, Chen LY. Critical review of acylation-stimulating protein physiology in humans and rodents. Biochim. Biophys. Acta, 2003. 1609: 127-43.

50. Yang X, Peterson L, Thieringer R, Deignan JL, Wang X, Zhu J, Wang S, Zhong H, Stepaniants S, Beaulaurier J, Wang IM, Rosa R, Cumiskey AM, Luo JM, Luo Q, Shah K, Xiao J, Nickle D, Plump A, Schadt EE, Lusis AJ, Lum PY. Identification and validation of genes affecting aortic lesions in mice. J. Clin. Invest., 2010. 120: 2414-22.

51. Sakuma M, Morooka T, Wang Y, Shi C, Croce K, Gao H, Strainic M, Medof ME, Simon DI. The intrinsic complement regulator decay-accelerating factor modulates the biological response to vascular injury. Arterioscler. Thromb. Vasc. Biol., 2010. 30: 1196-202.

52. Peng Q, Li K, Sacks SH, Zhou W. The role of anaphylatoxins C3a and C5a in regulating innate and adaptive immune responses. Inflamm. Allergy Drug Targets, 2009. 8: 236 


\section{Supplemental data}

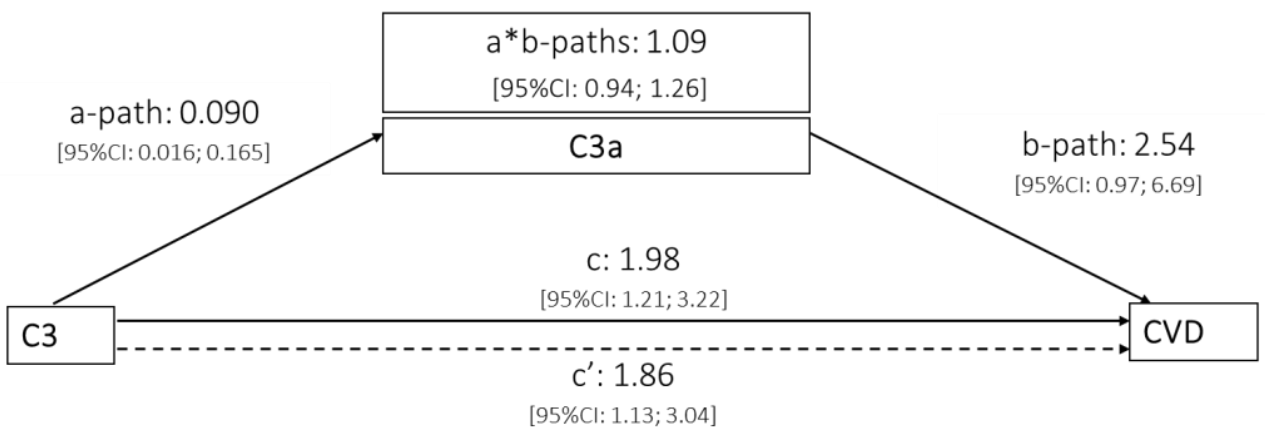

Supplemental figure 1: Mediation of the association of C3 with CVD in heavy smokers $(n=193)$ by C3a in a simple mediation model. The c-path represents the total effect of C3 on CVD, adjusted for covariates (see also table $3 \mathrm{~b}$, model 4). The a-path represents the association between $C 3$ and $C 3 a$, adjusted for covariates. The b-path represents the association between C3a and CVD, adjusted for C3 and covariates (see also table 3a, model 5). The $c^{\prime}$-path represents the direct effect of C3 on CVD and the a*b-path represents the indirect effect of C3 on CVD via C3a (see also table 3b, model 5). Due to different estimation procedures (ordinary least squares for the a-path and maximum likelihood for the $b$-path), the sum of $\left(a^{*} b+c^{\prime}\right)$ does not add to the c-path.

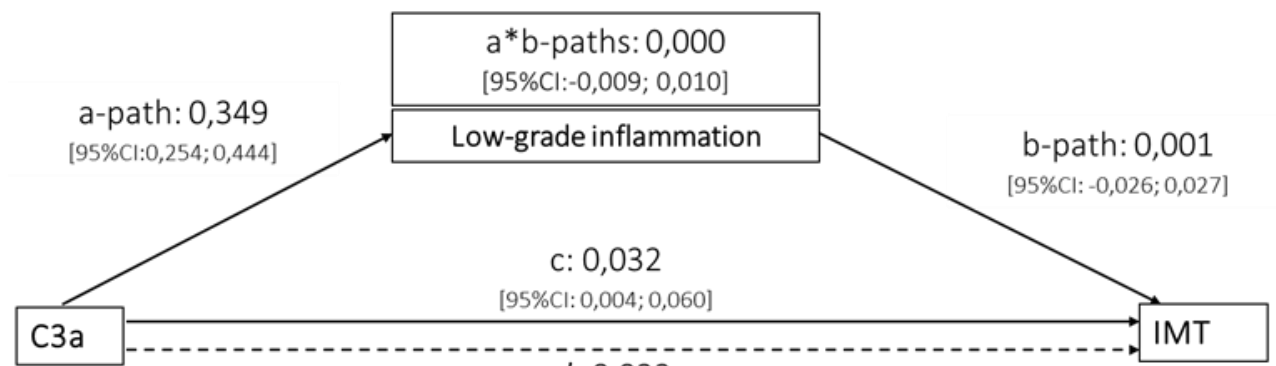

$$
c^{\prime}: 0,032
$$

[95\%Cl: 0,002; 0,061]

2a) Mediation of the association of C3a with cIMT by low-grade inflammation ( $N=504)$.

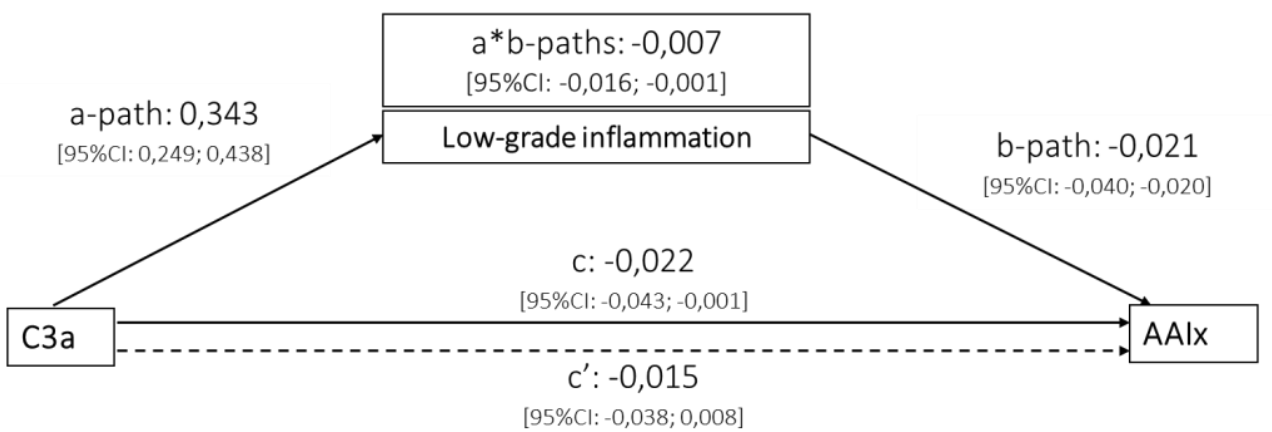

2b) Mediation of the association of C3a with AAlx by low-grade inflammation ( $N=541$ ). 


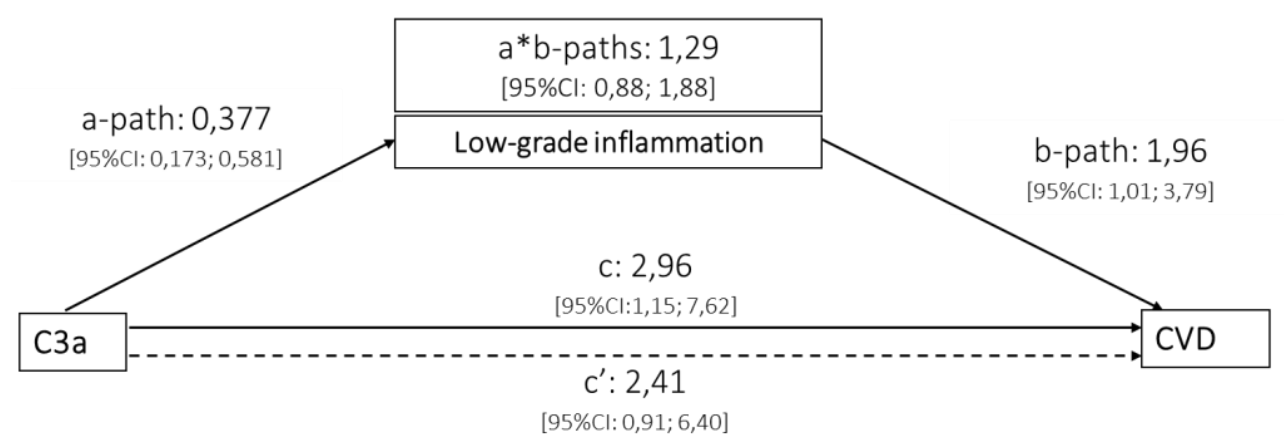

2c) Mediation of the association of C3a with CVD in heavy smokers by low-grade inflammation ( $N=193$ ).

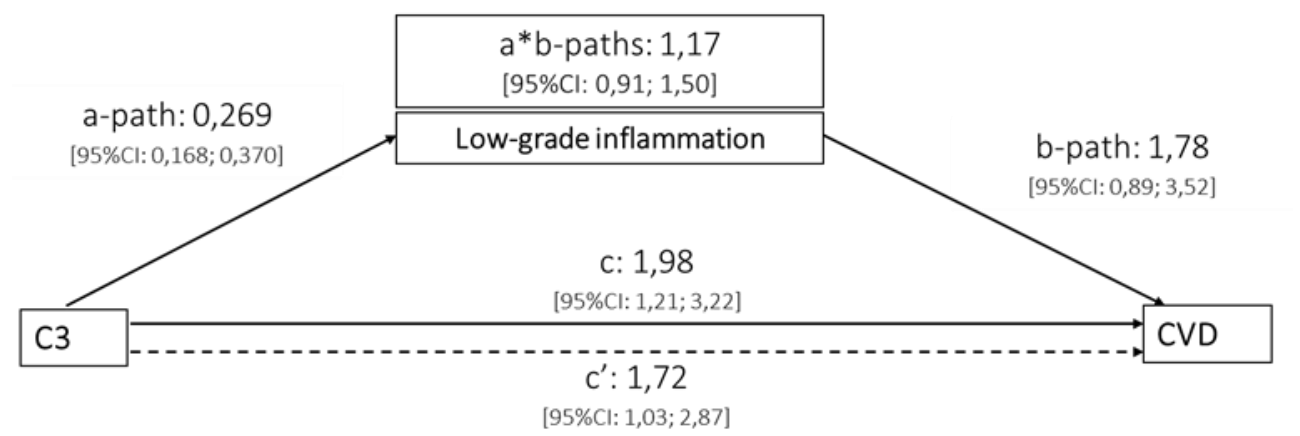

2d) Mediation of the association of C3 with CVD in heavy smokers by low-grade inflammation ( $N=193$ ).

Supplemental figure 2: Mediation of the associations of C3a and C3 by low-grade inflammation (calculated as the mean of the Z-scores of CRP, IL-6, TNF $\alpha$, IL-8, sICAM-1, SAA, ceruloplasmin and haptoglobin) in simple mediation models. The c-path represents the total effect of C3a or C3 on the outcome (cIMT, AAlx, CVD), adjusted for covariates (see also table $3 \mathrm{~b}$, model 4). The a-path represents the association between $\mathrm{C} 3 \mathrm{a}$ or $\mathrm{C} 3$ and low-grade inflammation, adjusted for covariates. The b-path represents the association between low-grade inflammation and the outcome (cIMT, AAlx, CVD), adjusted for C3a or C3 and covariates. The $c^{\prime}$-path represents the direct effect of C3a or C3 on the outcome (cIMT, AAIx, CVD), and the $a^{*}$ b-path represents the indirect effects of C3a or C3 on the outcome via low-grade inflammation. In the mediation models with CVD as outcome the sum of $\left(a^{*} b+c^{\prime}\right)$ does not add to the c-path due to different estimation procedures (ordinary least squares for the a-path and maximum likelihood for the b-path). 


\section{Chapter 5 CHSDfGr?}

Complement activation products $\mathrm{C} 5 \mathrm{a}$ and sC5b-9 are associated with low-grade inflammation and endothelial dysfunction, but not with atherosclerosis in a cross-sectional analysis: the CODAM study

published in parts in: International Journal of Cardiology 2014. 174: 400-3

E. Hertle, M.M.J. van Greevenbroek, I.C.W. Arts, C.J.H. van der Kallen, E.J.M. Feskens, C.G. Schalkwijk, C.D.A. Stehouwer 


\begin{abstract}
Complement activation products $\mathrm{C} 5 \mathrm{a}$ and soluble C5b-9 (sC5b-9) are increased in acute inflammation and in acute cardiovascular events. However, it is unknown whether they contribute to low-grade inflammation, endothelial dysfunction, atherosclerosis and/or stable cardiovascular disease (CVD).

We performed cross-sectional analyses among 537 individuals [61\% men, 59.4 46.9 years, $26 \%$ with type 2 diabetes, 28\% with CVD] of CODAM. Scores for low-grade inflammation and endothelial dysfunction were calculated by averaging the Z-scores of 8 biomarkers of lowgrade inflammation, and 3 biomarkers of endothelial dysfunction, respectively. Markers of atherosclerosis were carotid intima-media thickness (cIMT) and ankle-arm blood pressure index (AAIx). Prevalent CVD was assessed with self-reports, ECG examinations and/or $A A I x<0.9$. We conducted multiple linear and logistic regression analyses with adjustments for age, sex, glucose metabolism status, BMI, waist, lipids, blood pressure, renal function, physical activity, smoking and use of medication.

Per 1SD increase, C5a $(\beta(S D)=0.180$ [95\%Cl: 0.105; 0.255]) and sC5b-9 $(\beta(S D)=0.217$ [0.143; $0.292])$ were positively associated with low-grade inflammation. Also, C5a $(\beta(S D)=0.080$ [0.003; 0.156]) and sC5b-9 $(\beta(S D)=0.127[0.051 ; 0.203])$ were positively associated with endothelial dysfunction. In contrast, $\mathrm{C} 5 \mathrm{a}$ and $\mathrm{sC} 5 \mathrm{~b}-9$ were not associated with $\mathrm{CIMT}$, AAIx or CVD (P-value>0.15).

In conclusion, higher plasma C5a and sC5b-9 concentrations were independently associated with more systemic low-grade inflammation and more endothelial dysfunction. However, C5a and sC5b-9 were not associated with markers of atherosclerosis or CVD. Systemic activation of the terminal complement pathway may thus participate in a chronic inflammatory state and impair endothelial function, but is not increased in atherosclerosis or stable CVD.
\end{abstract}




\section{Introduction}

Low-grade inflammation plays a crucial role in all stages of cardiovascular disease (CVD), from the development of endothelial dysfunction and atherosclerosis to the acute event of atherothrombosis [1]. Inflammatory processes can be induced and regulated by the complement system, which is a complex protease network that orchestrates various immune, inflammatory and metabolic circuits [2]. Activation of the complement network generates multiple effectors in sequential stages, and activation of the terminal pathway, which generates complement $\mathrm{C} 5 \mathrm{a}$ and $\mathrm{C} 5 \mathrm{~b}-9$, is the final step in the complement cascade [3]. Both C5a and C5b-9 are thought to be involved in the pathophysiology of CVD [4].

The anaphylatoxin $\mathrm{C} 5 \mathrm{a}$ is a soluble mediator, which can signal via different receptors on a wide variety of immune and non-immune cells [5]. C5a is the most powerful of the known anaphylatoxins in inducing chemotaxis, immune and endothelial activation [6]. C5b-9, in turn, is a complex of complement proteins C5b, C6, C7, C8 and C9, and is also denoted terminal complement complex (TCC) or membrane attack complex (MAC). On the target membrane, C5b-9 forms a pore, and promotes cell lysis or intracellular signalling that activates inflammatory pathways, depending on the amount and size of pores formed [7-11]. Soluble C5b-9 (sC5b-9) is the circulating form of C5b-9, which is assumed to be formed simultaneously to membrane-bound C5b-9 and also to arise from membrane-shed C5b-9, consequently reflecting burden of terminal complement activation. Soluble terminal complement complexes can also induce intracellular signalling [12] and have been shown to induce vascular leakage [13] and endothelial activation [14] in vitro. Animal studies suggest a role of terminal complement activation in atherosclerosis, as inhibition of C5a signalling [15] or C6 deficiency (resulting in the inability to form C5b-9) [16, 17] reduced atherosclerosis. In humans, prospective studies in patients with advanced CVD or acute cardiovascular events have shown associations of systemic concentrations of $\mathrm{C} 5 \mathrm{a}$ and sC5b9 with adverse cardiovascular outcomes [18-22]. However, these previous findings were confined to more extreme situations of terminal complement activation, as they were obtained in the acute phase of a cardiovascular event or in patients with advanced and unstable CVD. Currently, it is unknown whether $\mathrm{C} 5 \mathrm{a}$ and sC5b-9 are also involved in chronic low-grade inflammation, endothelial dysfunction and atherosclerosis, and thereby contribute to the development of CVD.

In the present study, we investigated the associations of plasma $\mathrm{C5a}$ and sC5b-9 with systemic low-grade inflammation, endothelial dysfunction, markers of atherosclerosis [carotid intima-media thickness (cIMT), ankle-arm blood pressure index (AAIx)] and/or with prevalent CVD, representing progressive stages of CVD, in a cohort of middle-aged, Caucasian individuals with a moderately increased risk of type 2 diabetes (T2DM) and CVD - the CODAM study (Cohort study on diabetes and atherosclerosis Maastricht). Our last aim was to investigate whether potential associations of $\mathrm{C} 5 \mathrm{a}$ and $\mathrm{sC} 5 \mathrm{~b}-9$ with these cardiovascular outcomes are mediated via low-grade inflammation. 


\section{Methods}

\subsection{Study Participants}

This cross-sectional study was performed in the CODAM cohort [23]. Briefly, participants of a population-based cohort were invited for screening if they were Caucasian, aged $>40$ years, and met one of the following criteria: body mass index (BMI) $>25 \mathrm{~kg} / \mathrm{m}^{2}$; use of antihypertensive medication; positive family history of type 2 diabetes (T2DM); postprandial blood glucose $>6.0 \mathrm{mmol} / \mathrm{L}$; history of gestational diabetes, and/or glucosuria. The screening involved an oral glucose tolerance test (OGTT), and all those screened with T2DM or impaired glucose metabolism (IGM, combining impaired fasting glucose [IFG] and impaired glucose tolerance [IGT]) and a random selection of those with normal glucose metabolism (NGM) were invited to participate in CODAM ( $\mathrm{N}=574)$. The study was approved by the Medical Ethics Committee of the Maastricht University Medical Centre. All participants gave written informed consent.

In the current study, we excluded individuals with missing data in C5a or sC5b-9 (n=16), inflammatory markers $(n=2)$ or covariates $(n=19)$. Additionally, we excluded in the respective analyses of endothelial dysfunction, CIMT and AAlx individuals lacking data on endothelial dysfunction markers $(n=7)$, cIMT $(n=41)$, or individuals lacking data on the AAIx $(n=1)$ and those with AAlx values suggesting arterial calcification (AAl $\geq 1.5, n=3$ ) [24]. Thus, the associations with inflammation, endothelial dysfunction, AAlx and CVD were investigated in 530-537 individuals, while associations with cIMT were investigated in 496 individuals.

\subsection{Laboratory measurements}

Participants were asked to stop lipid-modifying medication 14 days prior to blood withdrawal, while other medication was stopped one day beforehand. Blood samples were collected after an overnight fast by venipuncture. EDTA and citrate tubes were kept on ice until blood was centrifuged at $4^{\circ} \mathrm{C}$ (within 3 hours). Serum was allowed to clot at room temperature for 45 minutes. Aliquots were stored at $-80^{\circ} \mathrm{C}$ until use.

Complement factors: Aliquots were thawed rapidly at $37^{\circ} \mathrm{C}$ and subsequently transferred to ice. Complement C5a was measured in EDTA plasma by ELISA (MicroVue C5a EIA kit, Quidel, Catalogue number A021, San Diego, USA). sC5b-9 was measured in citrate plasma by ELISA (MicroVue sC5b-9 EIA kit, Quidel, Catalogue number A029). This assay uses anti-C9neoepitope antibodies and will as such not be able to distinguish between SC5b-9 (soluble C5b-9 complexes containing inhibitory proteins such as S-protein), sC5b-9 (soluble C5b-9) and plasma MAC (plasma membrane vesicles containing membrane-bound C5b-9), which all reflect activation of the terminal complement pathway [25]. Inter-assay coefficients of variation (CV) were $5.3 \%$ for $\mathrm{C} 5 \mathrm{a}$ and $11.8 \%$ for sC5b-9.

Inflammatory markers: Interleukin-8 (IL-8) and tumour necrosis factor- $\alpha$ (TNF $\alpha$ ) were measured in EDTA plasma using a multiarray detection system (MesoScale Discovery, SECTOR Imager 2400, Gaithersburg, Maryland, USA). C-reactive protein (CRP), interleukin-6 (IL-6), soluble intercellular adhesion molecule-1 (sICAM-1) and serum amyloid A (SAA) were measured twice, once in serum or EDTA plasma with ELISA [26] and once in EDTA plasma 
using a multiarray detection system (MesoScale Discovery, SECTOR Imager 2400), and were calibrated after cross-validation as described in [27]. Haptoglobin and ceruloplasmin were measured in serum by an automatic analyser using Tina-quant assay (Roche diagnostics) [28]. CVs varied between $2 \%$ and $9 \%$, except for SAA (CV was 12\%).

Endothelial dysfunction markers: Von Willebrand factor (VWF) was measured with an inhouse ELISA in citrated plasma as described [29], and the concentration was expressed as percentage of antigen concentration in normal pooled plasma. Soluble E-selectin (SEs) and soluble vascular cell adhesion molecule-1 (sVCAM-1) were measured twice, once in serum or EDTA plasma with ELISA [30] and once in EDTA plasma using a multiarray detection system (MesoScale Discovery, SECTOR Imager 2400), and were calibrated after cross-validation as described in [27]. soluble Thrombomodulin (sTm) was measured using the multiarray detection system described. CVs varied between 5 and $6 \%$.

Cardiovascular measures: The cardiovascular outcomes were measured as previously published [30, 31]. cIMT was measured with an ultrasound imaging device (Ultramark 4+, Advance Technology Laboratories, Bothel, Washington, USA) that was placed $10-20 \mathrm{~mm}$ proximal to the carotid bulb. Measurements were obtained seven times at both the left and right common carotid artery. Mean cIMT was calculated from the medians of up to seven measurements of each side. For the AAIx, blood pressures were recorded once in the brachial arteries and twice in both the posterior tibial and dorsalis pedis arteries using a handheld 8-MHZ Doppler device (Mini Dopplex D900, currently: E-medical, Harmelen, The Netherlands). For each leg, the highest systolic pressure of the ankles was divided by the highest systolic brachial pressure. The lowest AAlx of either leg was used [32]. CVD was defined as the occurrence of at least one of the following: previous myocardial infarction (MI); coronary bypass; percutaneous coronary intervention or stroke reported by questionnaires; signs on a 12-lead electrocardiogram of MI (Minnesota codes 1-1 or 1-2) or ischaemia (Minnesota codes 1-3, 4-1, 4-2, 4-3, 5-1, 5-2, 5-3 or 7-1); non-traumatic limb amputation; and/or an AAlx <0.9.

Other covariates: NGM, IFG, IGT or T2DM were identified with a standard $75 \mathrm{~g}$ OGTT according to the WHO criteria of 1999 [33], and IFG and IGT were combined to IGM. Waist, $\mathrm{BMI}$ and blood pressure were measured by a research assistant [34]. Use of medication, smoking behaviour [34] and physical activity [35] were assessed with on-site administered questionnaires. Estimated glomerular filtration rate (eGFR) was calculated with the MDRD formula [36] from creatinine levels, measured in EDTA plasma with a Jaffé diagnostic test (Roche Diagnostics). High-density lipoprotein cholesterol, total cholesterol and triacylglycerols were measured in EDTA plasma [34]. Low-density lipoprotein cholesterol was estimated with the Friedewald formula [37].

\subsection{Statistical analyses}

Variables were visually inspected for normal distribution. Normally distributed variables are presented as mean \pm standard deviation (SD). Skewed variables are presented as medians

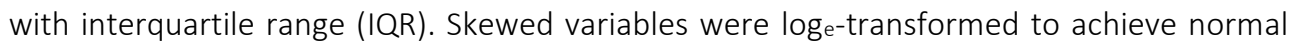
distribution prior to the analyses. One-way ANOVA was used to compare continuous 
variables, and Pearson $\mathrm{Chi}^{2}$ to compare categorical variables across tertiles of $\mathrm{C} 5 \mathrm{a}$ or sC5b-9 concentrations. To provide a robust estimate of inflammation and endothelial dysfunction with minimization of random error, composite scores were calculated by averaging the Zscores (Z-score: [individual value minus mean of all values]/SD of all values) of CRP, IL-6, IL-8, TNF $\alpha$, sICAM-1, SAA, haptoglobin and ceruloplasmin for inflammation [28], and the Z-scores of VWF, sEs and SVCAM-1 for endothelial dysfunction [30]. These composite scores were converted to their standardized scores afterwards. To allow for a direct comparison of the effect sizes, also C5a, sC5b-9, cIMT and AAlx were converted to their standardized scores. Consequently, in all regression analyses the associations are presented per 1 SD increase in $\mathrm{C} 5 \mathrm{a}$ or $\mathrm{sC5b-9}$, and in the linear regression analyses, outcomes (inflammation, endothelial dysfunction, cIMT, AAIx) are also expressed in SDs.

Linear and logistic regression analyses: Multiple linear regression was used to investigate the associations of $\mathrm{C} 5 \mathrm{a}$ and sC5b-9 with inflammation (aim 1) and endothelial dysfunction, cIMT and AAIx (aim 2). Multiple logistic regression was used to investigate the associations of C5a and sC5b-9 with prevalent CVD (aim 2). First we adjusted for variables that are related to the inclusion procedure, which incorporated stratified sampling for age and sex, and oversampling for IGM and T2DM [model 1]. Next, we additionally adjusted for measures of obesity (BMI, waist) [model 2] as adipose tissue can express both complement and inflammatory factors $[38,39]$. Finally, in the fully adjusted model, we additionally adjusted for cardiovascular risk factors and drug use that might affect inflammatory and immunological processes (triacylglycerols, total cholesterol, high-density lipoprotein cholesterol, SBP, eGFR, smoking, physical activity and use of anti-hypertensive or glucoselowering medication) [model 3].

Next, we performed mediation analyses to investigate the role of inflammation in the associations of $\mathrm{C} 5 \mathrm{a}$ and sC5b-9 (aim 3). For this, we first evaluated the association of lowgrade inflammation itself with the cardiovascular measures. Next, we conducted mediation analyses as described elsewhere [40], to determine the extent to which the association(s) of $\mathrm{C} 5 \mathrm{a}$ and sC5b-9 with cardiovascular measures, if present, were mediated (i.e. explained) by inflammation. In short, we estimated the indirect effect of $\mathrm{C} 5 \mathrm{a}$ and sC5b-9 on the outcome via inflammation and computed bootstrapped (bias-corrected) confidence intervals (5000 samples) for the size of the mediated effect, using SPSS macros provided by Preacher and Hayes [41].

Additional analyses: To substantiate the robustness of our observations, we performed several additional analyses. First, we analysed the associations of $\mathrm{C} 5 \mathrm{a}$ and sC5b-9 with endothelial dysfunction when sICAM-1, and when both sICAM-1 and sTm were included in the endothelial dysfunction score. sICAM-1 is widely used as marker of inflammation, but as both monocytes and endothelial cells express SICAM-1 it can also be regarded as marker of endothelial dysfunction [42]. For sTm, both in vitro and human studies so far yielded contradictory findings whether higher or lower concentrations reflect endothelial dysfunction [43]. Next, we adjusted all fully adjusted associations for use of lipid-modifying medication, which might in cross-sectional analysis act both as a collider and as a confounder. A collider is defined as a common effect of exposure (or of causes of exposure) and outcome 
[44]. As lipid-modifying medication is in practice often prescribed after a clinical event regardless of actual lipid profiles it can be considered a consequence of disease. Adjustment for such a collider might generate bias, while failure to adjust might result in confounding. Then, we repeated all analyses with exclusion of participants with a possible acute inflammation (serum CRP concentrations $>10 \mathrm{mg} / \mathrm{L}, \mathrm{n}=37$ ). Last, we analysed the associations with AAlx with exclusion of participants with peripheral arterial disease (PAD, $n=29)$ and also with exclusion of participants with an AAlx higher than $1.3(n=17)$, as 1.3 is discussed as lowest cut-off for arterial calcification [24].

Assumptions for linear and logistic regression analyses were met. Statistical analyses were performed using the SPSS package version 20.0 (SPSS, Chicago, IL, USA) and statistical significance was set at $\mathrm{P}<0.05$.

\section{Results}

\subsection{General characteristics of the study population}

Plasma C5a concentrations ranged from 1.2 to $20.5 \mu \mathrm{g} / \mathrm{L}$, with a mean $\pm \mathrm{SD}$ of $7.6 \pm 3.9 \mu \mathrm{g} / \mathrm{L}$. Plasma sC5b-9 concentrations ranged from 32 to $272 \mu \mathrm{g} / \mathrm{L}$, with a mean \pm SD of $113 \pm 34 \mu \mathrm{g} / \mathrm{L}$. C5a and sC5b-9 concentrations were significantly but weakly correlated (Pearson's correlation coefficient $0.13, \mathrm{P}=0.002$ ). Table 1 shows characteristics of the study population as a whole and across tertiles of plasma $\mathrm{C} 5 \mathrm{a}$ and $\mathrm{sC} 5 \mathrm{~b}-9$ concentrations. Higher tertiles of C5a and sC5b-9 were characterized by a larger proportion of women, but did not show relevant differences in most other general characteristics, such as age, blood pressure, blood lipids or use of medication. In increasing tertiles of $\mathrm{C} 5 \mathrm{a}$, only $\mathrm{HbA} 1 \mathrm{c}$ and the portion of subjects with an abnormal glucose metabolism were higher, and the highest tertile of sC5b9 was characterized by higher BMI and lower physical activity. Most inflammatory markers and $\mathrm{VWF}$ were increasing in the higher tertiles of $\mathrm{C} 5 \mathrm{a}$ and/or sC5b-9, but there were no differences in CIMT, AAlx or prevalent CVD. 


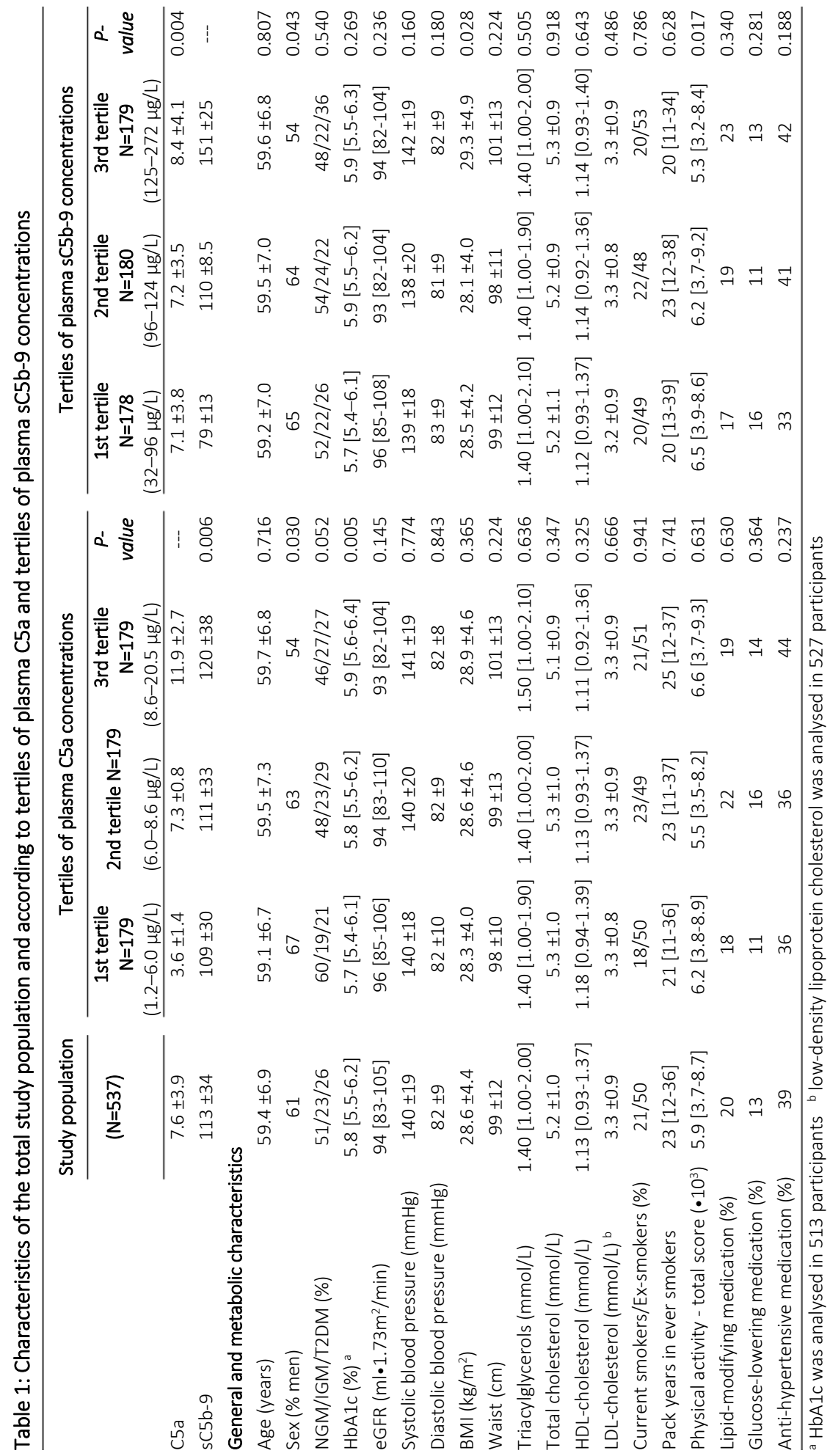




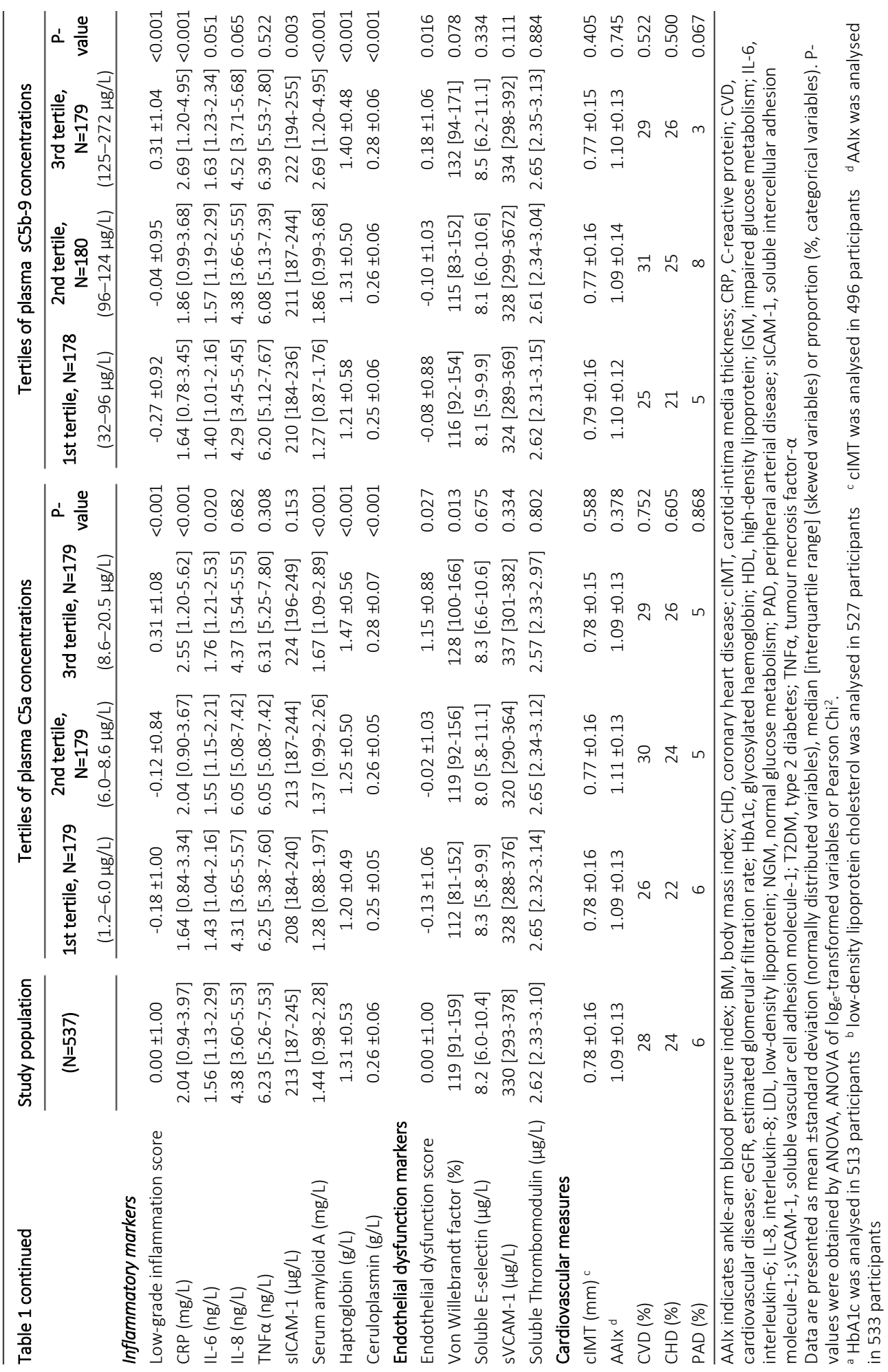




\subsection{Associations of $\mathrm{C} 5 \mathrm{a}$ and sC5b-9 with low-grade inflammation}

Plasma C5a and sC5b-9 were positively associated with the low-grade inflammation score (table 2, model 1). Additional adjustment for relevant covariates (models 2 and 3 ) only slightly attenuated these associations. In the fully adjusted model (model 3), one SD increase in C5a and SC5b-9 were associated with 0.180 SD [0.105; 0.255] and 0.217 SD [0.143; 0.292] higher inflammation score, respectively. In the analyses of the individual inflammatory markers (figure 1), all associations of C5a with inflammatory markers were positive except the association with IL-8, with significant associations for CRP, IL-6, SAA, haptoglobin, and ceruloplasmin. All associations of sC5b-9 with inflammatory markers were positive, with significant associations for all markers except for TNF $\alpha$.

Table 2: Associations of $\mathrm{C} 5 \mathrm{a}$ and sC5b-9 with low-grade inflammation

Association with low-grade inflammation ( $N=537$ )

\begin{tabular}{|c|c|c|}
\hline & $\beta$ for the inflammatory score $[95 \% \mathrm{Cl}]$ & P-value \\
\hline \multicolumn{3}{|c|}{ C5a (effect on the inflammatory score (in SD) per 1SD increase in C5a) } \\
\hline model 1 & $0.205[0.125 ; 0.285]$ & $<0.001$ \\
\hline model 2 & $0.189[0.111 ; 0.267]$ & $<0.001$ \\
\hline model 3 & $0.180[0.105 ; 0.255]$ & $<0.001$ \\
\hline \multicolumn{3}{|c|}{ sCb-9 (effect on the inflammatory score (in SD) per 1SD increase in sC5b-9) } \\
\hline model 1 & $0.238[0.159 ; 0.317]$ & $<0.001$ \\
\hline model 2 & $0.231[0.155 ; 0.308]$ & $<0.001$ \\
\hline model 3 & $0.217[0.143 ; 0.292]$ & $<0.001$ \\
\hline
\end{tabular}

model 1: adjusted for age [years]; sex [men/women]; impaired glucose metabolism [yes/no]; type 2 diabetes [yes/no]

model 2: model $1+$ body mass index $\left[\mathrm{kg} \bullet\left(\mathrm{m}^{2}\right)^{-1}\right]$; waist $[\mathrm{cm}]$

model 3: model $2+$ triacylglycerols [mmol/L]; total cholesterol $[\mathrm{mmol} / \mathrm{L}]$; high-density lipoprotein cholesterol [mmol/L]; systolic blood pressure $[\mathrm{mmHg}]$; eGFR $\left[\mathrm{ml} \bullet 1.73 \mathrm{~m}^{2} \bullet(\mathrm{min})^{-1}\right]$; smoking [pack years]; physical activity [total score]; use of glucose-lowering medication [yes/no]; use of anti-hypertensive medication [yes/no]

Skewed variables were $\log _{\mathrm{e}}$ transformed prior to the analyses. 


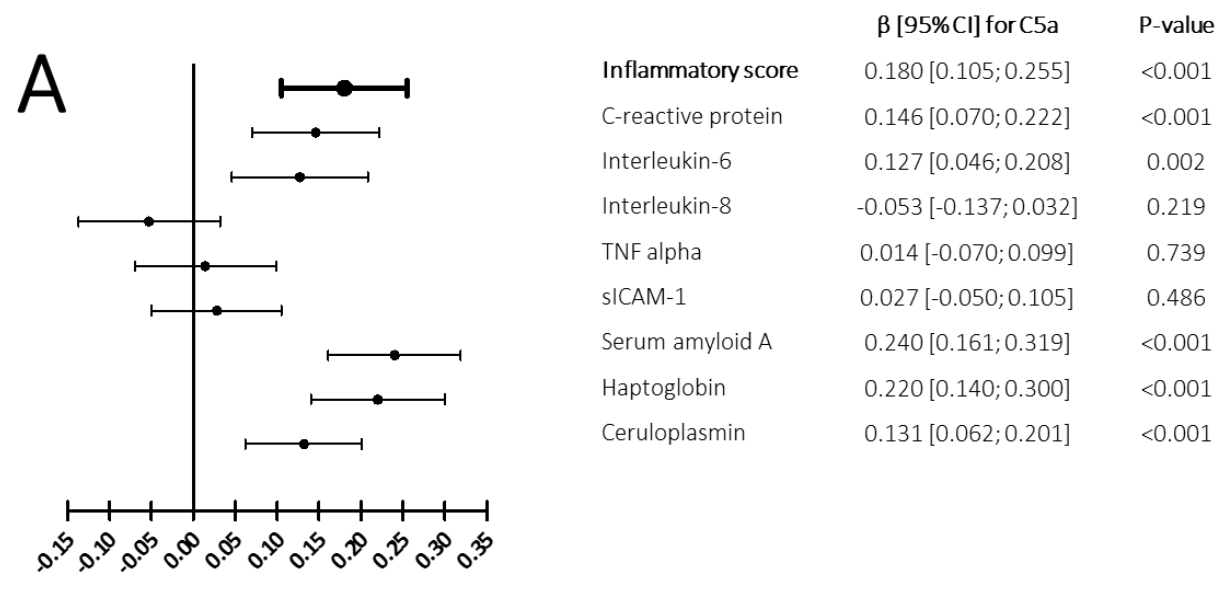

$\beta[95 \% \mathrm{Cl}]$ for the fully adjusted associations of C5a with markers of low-grade inflammation

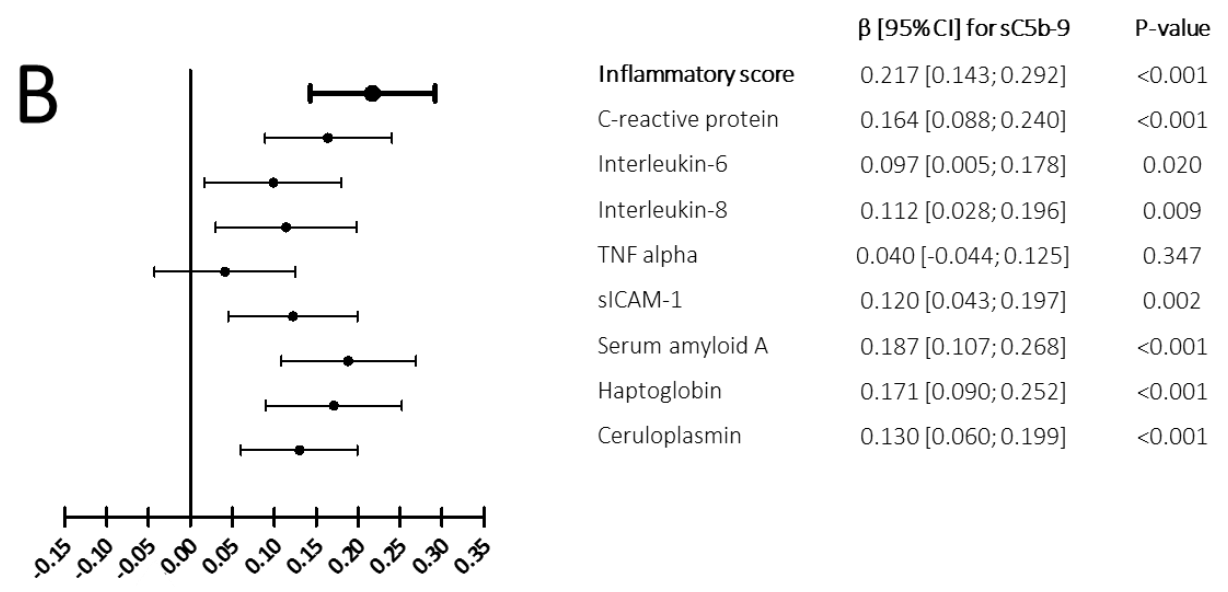

$\beta[95 \% \mathrm{Cl}]$ for the fully adjusted associations of sC5b-9 with markers of low-grade inflammation

Figure 1: Associations of $\mathrm{C5a}$ (A) and sC5b-9 (B) with the inflammatory score and the individual inflammatory markers. Associations were adjusted for age, sex, impaired glucose metabolism, type 2 diabetes mellitus, body mass index, waist, systolic blood pressure, blood lipids (total cholesterol, high-density lipoprotein cholesterol, triacylglycerols), use of medication (glucose-lowering medication, anti-hypertensive medication), smoking (pack years), kidney function and physical activity. 


\subsection{Associations of C5a and sC5b-9 with cardiovascular measures}

C5a and sC5b-9 were positively associated with the endothelial dysfunction score, but were not associated with any of the other cardiovascular outcomes (table 3, model 1). The positive associations of $\mathrm{C} 5 \mathrm{a}$ and $\mathrm{sC5b}-9$ with the endothelial dysfunction score were only slightly attenuated and remained significant upon further adjustment (model 3, $\beta$ for $\mathrm{C} 5 \mathrm{a}$ and sC5b9 were $0.080[0.003 ; 0.156]$ and $0.127[0.051 ; 0.203]$ ). In the analysis of the individual endothelial dysfunction markers (figure 2), there was no association of C5a with sEs and sVCAM-1, but C5a was positively and significantly associated with vWF. For sC5b-9, the associations with VWF, sEs and SVCAM-1 were all positive, and significant or borderline significant.

In contrast, $\mathrm{C} 5 \mathrm{a}$ and sC5b-9 were not associated with cIMT (model 3, $\beta$ s for C5a and sC5b-9 were -0.006 [-0.090, 0.078] and -0.059 [-0.142, 0.023], respectively). Likewise, C5a and sC5b9 were not associated with AAlx (model 3, Bs were $0.041[-0.038,0.120]$ and $0.024[-0.054$, 0.103], respectively). Also, C5a and sC5b-9 were not associated with prevalent CVD (model 3 , ORs were $0.92[0.75,1.14]$ and $0.98[0.79,1.21]$, respectively).

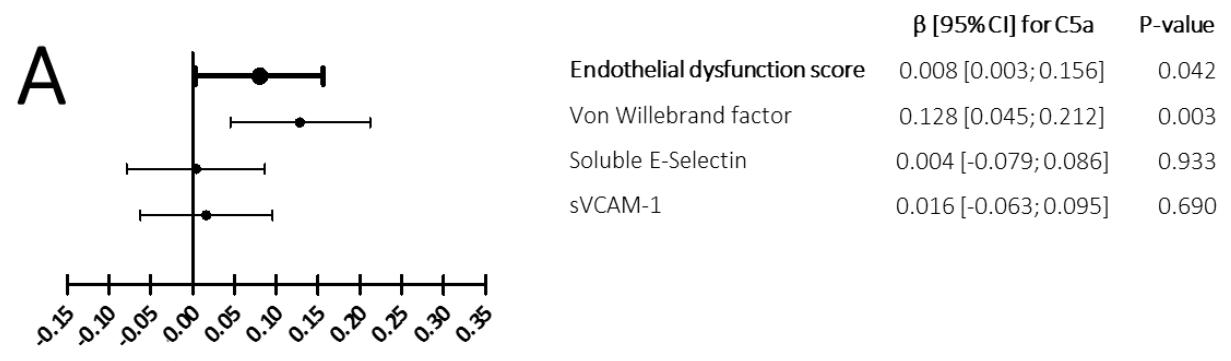

$\beta[95 \% \mathrm{Cl}]$ for the fully adjusted associations of $\mathrm{C5}$ a with markers of endothelial dysfunction

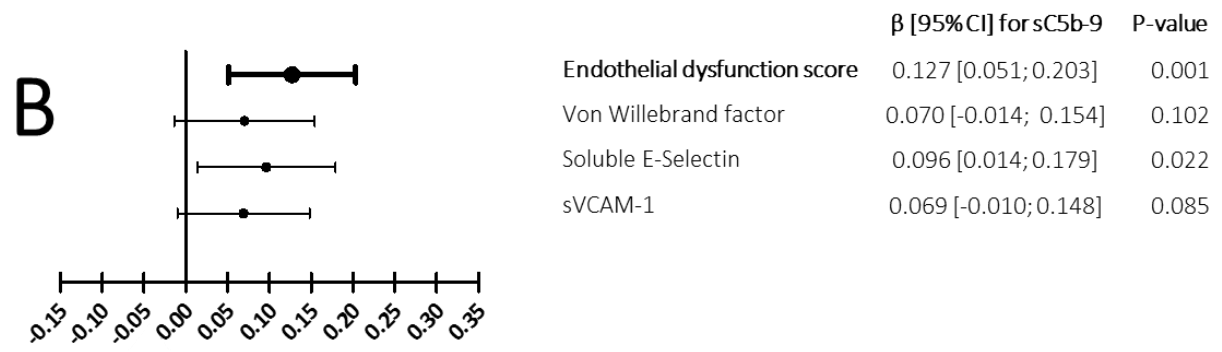

$\beta[95 \% \mathrm{Cl}]$ for the fully adjusted associations of sC5b-9 markers of endothelial dysfunction

Figure 2: Associations of $\mathrm{C} 5 \mathrm{a}(\mathrm{A})$ and $\mathrm{sC} 5 \mathrm{~b}-9$ (B) with the endothelial dysfunction score and the individual markers of endothelial dysfunction. Associations were adjusted for age, sex, impaired glucose metabolism, type 2 diabetes mellitus, body mass index, waist, systolic blood pressure, blood lipids (total cholesterol, high-density lipoprotein cholesterol, triacylglycerols), use of medication (glucose-lowering medication, anti-hypertensive medication), smoking (pack years), kidney function and physical activity. 


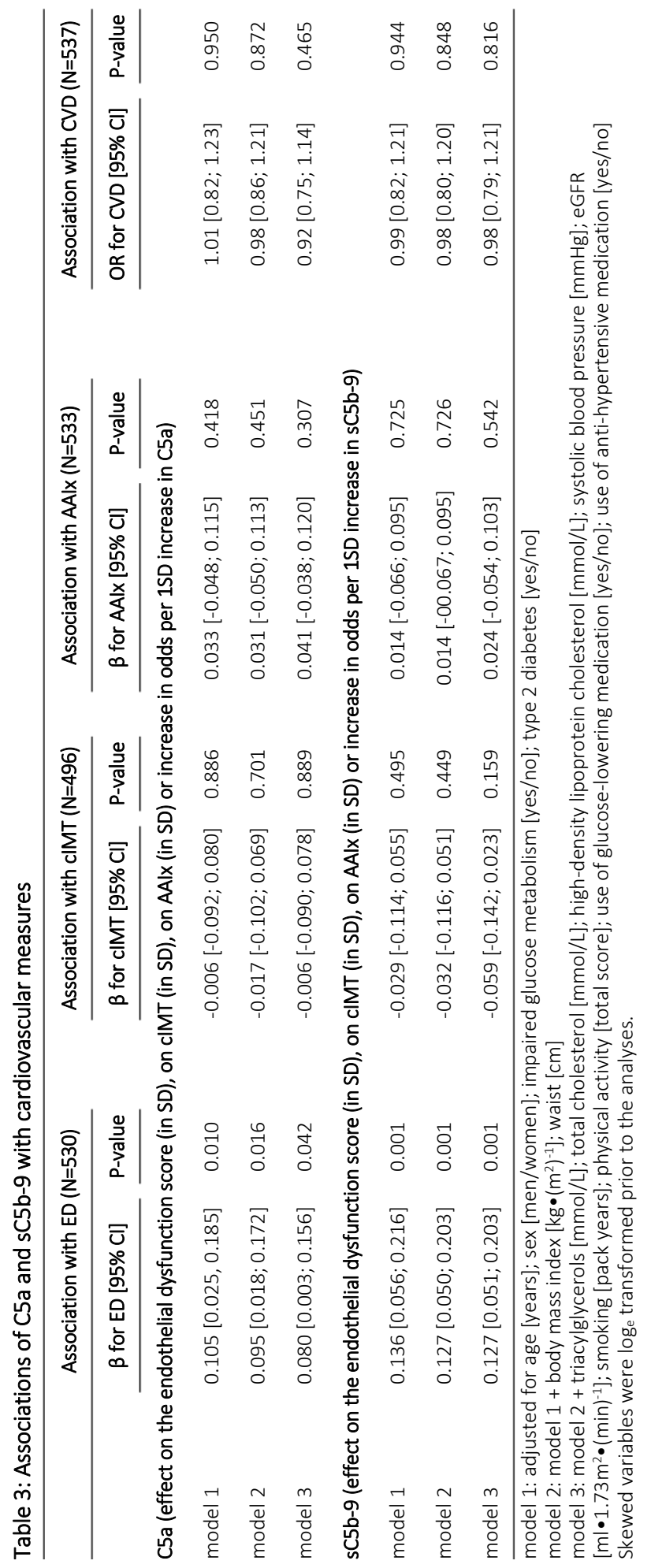




\subsection{Low-grade inflammation in the associations of $\mathrm{C} 5 \mathrm{a}$ and sC5b-9 with cardiovascular measures}

The low-grade inflammation score itself was positively associated with the endothelial dysfunction score, was positively associated with CIMT and inversely associated with the AAlx, both reflecting more atherosclerosis, and was also positively associated with prevalent CVD (supplemental table, model 1). These associations remained significant upon further adjustment, except for the association of low-grade inflammation with cIMT, which was attenuated and became non-significant (supplemental table, model 3).

In mediation analyses, addition of the low-grade inflammation score to the fully adjusted associations of $\mathrm{C} 5 \mathrm{a}$ and $\mathrm{sC} 5 \mathrm{~b}-9$ with the endothelial dysfunction score strongly attenuated the regression coefficients of $\mathrm{C} 5 \mathrm{a}$ and $\mathrm{sC5b}-9$. The direct effects of $\mathrm{C} 5 \mathrm{a}$ and $\mathrm{sC} 5 \mathrm{~b}-\mathrm{9}$ on endothelial dysfunction not mediated via inflammation were 0.030 [-0.045; 0.105] and 0.068 [-0.008; 0.143], respectively. Low-grade inflammation significantly explained $63 \%$ of the association of $\mathrm{C} 5 \mathrm{a}$ with the endothelial dysfunction score (indirect effect of C5a via low-grade inflammation: $\beta$ was 0.050 [0.028; 0.083]), and significantly explained $46 \%$ of the association of sC5b-9 with the endothelial dysfunction score (indirect effect of sC5b-9 via low-grade inflammation: $\beta$ was 0.059 [0.034; 0.098]).

Addition of the low-grade inflammation score to the models with CIMT, AAlx and CVD as outcome did not alter these non-significant associations (data not shown).

\subsection{Additional analyses}

When sICAM-1 was additionally included in the endothelial dysfunction score, the associations of $\mathrm{C} 5 \mathrm{a}$ and $\mathrm{sC} 5 \mathrm{~b}-9$ with endothelial dysfunction were not materially altered (model 3, $\beta$ (C5a) was 0.068 [-0.008; 0.143], $P=0.080$ and $\beta$ (sC5b-9) was 0.140 [0.064; 0.215], $P<0.001)$. When both sICAM-1 and sTm were included in the endothelial dysfunction score, the associations of $\mathrm{C} 5 \mathrm{a}$ and $\mathrm{sC5b}-9$ with the endothelial dysfunction score were partly attenuated (model $3, \beta$ (C5a) was 0.052 [-0.025; 0.129], $P=0.187$ and $\beta$ (sC5b-9) was 0.116 [0.039; 0.193], $P=0.003$ ). C5a nor sCb5-9 was associated with sTm (model 3, $\beta$ (C5a) was 0.017 [-0.097; 0.063], $\mathrm{P}=0.679$ and $\beta$ (sC5b-9) was -0.008 [0-0.088; 0.072], $\mathrm{P}=0.850$ ).

Additional adjustment for use of lipid-modifying medication did not materially alter the associations of $\mathrm{C} 5 \mathrm{a}$ and $\mathrm{SC} 5 \mathrm{~b}-9$ with the low-grade inflammation score, the endothelial dysfunction score, cIMT or AAIx, and also did not alter the non-significant associations of C5a and sC5b-9 with prevalent CVD (data not shown). When participants with CRP concentrations above $10 \mathrm{mg} / \mathrm{L}(\mathrm{N}=37$ ) were excluded, the associations of $\mathrm{C} 5 \mathrm{a}$ and SC5b-9 with the low-grade inflammation score were attenuated but remained highly significant (model 3, $\beta$ (C5a) was 0.097 [0.026; 0.168], $P=0.008$ and $\beta$ (sC5b-9) was 0.159 [0.089; 0.229], $P<0.001)$. The strength of association of $\mathrm{C} 5$ a with endothelial dysfunction increased (model $3, \beta$ was 0.104 [0.023; 0.186], $P=0.012$ ) while the strength of the association of $s C 5 b-9$ with endothelial dysfunction decreased ( $\beta$ was 0.093 [0.011; 0.175], $P=0.025$ ). The non-significant associations of C5a and SC5b-9 with cIMT, AAlx and prevalent CVD were not materially altered upon exclusion of subjects with elevated CRP (data not shown). When participants 
with PAD or an AAlx above 1.3 were excluded from the analyses, the non-significant associations of $\mathrm{C} 5 \mathrm{a}$ and sC5b-9 with the AAlx were not materially altered (data not shown).

\section{Discussion}

This is the first relatively large human study that has evaluated the independent associations of circulating $\mathrm{C} 5 \mathrm{a}$ and sC5b-9 with low-grade inflammation and different manifestations of CVD. There were three main findings. First, higher plasma concentrations of C5a and sC5b-9 were associated with increased systemic low-grade inflammation, independently of a large number of confounders. Second, higher concentrations of C5a and sC5b-9 were associated with more endothelial dysfunction. These associations were also independent of a large number of confounders, and were for a substantial part explained by low-grade inflammation. Third, C5a and sC5b-9 were not associated with markers of atherosclerosis or prevalent CVD.

No prior studies on $\mathrm{C} 5 \mathrm{a}$ and sC5b-9 in chronic inflammation have been published, although their role in acute inflammatory processes is well-known. Both C5a and sC5b-9 increase during sepsis [45], and C5a has been implicated in specific inflammatory responses such as in allergic asthma, rheumatoid arthritis or multiple sclerosis [6]. Moreover, C5a was shown to regulate inflammation in adipose tissue in mice [46] and in vitro studies showed that C5a and sC5b-9 can induce cytokines and chemokines in neutrophils, monocytes, macrophages, endothelial cells and hepatic stellate and Kupffer cells [2, 11, 25, 47, 48]. Our current findings indicate that $\mathrm{C} 5 \mathrm{a}$ and $\mathrm{sC5b}-9$ may not only contribute to the above-mentioned specific inflammatory responses, but may in humans also participate in chronic low-grade inflammation. We averaged eight biomarkers of low-grade inflammation to obtain a robust estimate of inflammation, and showed that $\mathrm{C} 5 \mathrm{a}$ and $\mathrm{sC} 5 \mathrm{~b}-9$ were associated with this overall inflammatory state. Also, $\mathrm{C} 5 \mathrm{a}$ and $\mathrm{sC} 5 \mathrm{~b}-9$ were consistently associated with most individual markers CRP, IL-6, IL-8, TNF $\alpha$, sICAM-1, SAA, haptoglobin and ceruloplasmin. This suggests that $\mathrm{C} 5 \mathrm{a}$ and $\mathrm{sC} 5 \mathrm{~b}-9$ may participate in multiple inflammatory processes in different organs, such as in the endothelium, in the liver and in adipose tissue, and thereby contribute to systemic low-grade inflammation. Our findings remained highly significant when we excluded participants with elevated CRP (>10 mg/L) concentrations, which underscores that the associations we observed indeed represent an association with low-grade inflammation. C5a and sC5b-9 were also positively associated with plasma markers of endothelial dysfunction, but this association was weaker than with low-grade inflammation. Also, the association with endothelial dysfunction was less consistent among the individual markers, as sC5b-9 was associated with overall endothelial dysfunction, while C5a was associated only with vWF. C5a and SC5b-9 may contribute to endothelial dysfunction via on the one hand direct effects on the endothelium and on the other hand indirectly via stimulation of systemic low-grade inflammation. In the mediation analysis, we showed that $46-63 \%$ of the associations of $\mathrm{C} 5 \mathrm{a}$ and sC5b-9 with the endothelial dysfunction score was mediated via inflammation, leaving a minor part of this association independent of inflammation. This is in line with a potential role for both direct and indirect effects of $\mathrm{C} 5 \mathrm{a}$ and sC5b-9 on the 
endothelium. The endothelium is directly influenced by humoral cytokines, chemokines and metabolites, and is constantly exposed to systemic C5a and sC5b-9 concentrations. Several in vitro studies showed that $\mathrm{C} 5 \mathrm{a}$ and $\mathrm{sC} 5 \mathrm{~b}-9$ can induce different processes of endothelial activation [7, 9, 14, 49, 50]. Moreover, C5a has been shown to release vWF [51] from endothelial cells and sC5b-9 to induce VCAM-1 [52]. Of note, plasma markers of endothelial dysfunction are considered to not only reflect the large vessels that are affected in atherosclerosis and CVD, but also to reflect the microvasculature [53]. The microvasculature represents the greatest part of the total vascular surface area and is therefore suggested to contribute the most part to circulating endothelial markers. C5a and sC5b-9 might thus potentially be involved in microvascular impairments given their associations with these endothelial markers. In contrast, $\mathrm{C} 5 \mathrm{a}$ and $\mathrm{sC5b}-9$ were not associated with additional cardiovascular measures cIMT and AAlx. Notably, these measures of atherosclerosis are estimates of large artery impairments, and in contrast to the endothelial markers discussed above, do not reflect microvascular impairments. This lack of an association with cIMT and AAlx was surprising, given previous studies that have implicated terminal complement activation in atherosclerosis. In humans, no previous studies have been published on systemic levels of C5a and C5b-9 and severity of atherosclerosis, but C5a and sC5b-9 were detected in atherosclerotic plaques [54]. In animals, C5a and/or (s)C5b-9 have been implicated in atherosclerosis in some [15-17] but not all [55] studies, using cholesterol-fed rabbits or $\mathrm{ApoE}^{-/-}$or $\mathrm{LDL}^{-/-}$mice. Furthermore, (s)C5b-9 was shown to activate vascular smooth muscle cells [8]. Of note, cellular processes of atherosclerosis occur in a locally confined fashion, and sub-endothelial and intra-plaque generation of C5a and sC5b-9 may in atherosclerosis be more relevant [54] than systemic complement activation. Our findings do not preclude that $\mathrm{C} 5 \mathrm{a}$ and $\mathrm{sC} 5 \mathrm{~b}-9$ may play a role at the sites of atherosclerosis, rather they indicate that their systemic concentrations do not appear to be related to atherosclerosis.

C5a and sC5b-9 were not associated with prevalent CVD. We have no reasons to believe that any methodological shortcoming may have contributed to these null associations. We have in the CODAM Study previously shown positive associations of inflammation and endothelial dysfunction with AAIx and CVD [26, 30] and the observed associations of inflammation with endothelial dysfunction and cIMT were also as expected. Previous human studies on C5a and sC5b-9 have demonstrated that their levels rise in acute cardiovascular events, where they are thought to mediate ischaemia/reperfusion-injury [56]. In addition, levels of sC5b-9 at admission for acute $\mathrm{Ml}$ or stroke were associated with stroke severity or with worse outcome $[21,57]$, but post-admission or follow-up values were not [21]. Furthermore, C5a was, in patients with advanced PAD or patients undergoing interventions to restore blood flow in peripheral or coronary arteries, positively associated with second CVD events or restenosis [18-20]. These [18-21, 57] and other studies [58, 59] suggest that systemic C5a and sC5b-9 concentrations reflect the severity of active disease processes in advanced CVD or in acute cardiovascular events. The greatest difference compared to our study may be the study population used. These previous studies [18-21, 57-59] all included hospital patients with acute or advanced CVD. In contrast, our study population comprises individuals with stable and milder forms of CVD, as our study participants originate from the general population and 
as we included silent $\mathrm{MI}$ (assessed with ECG) in our definition of CVD. Our finding that systemic levels of C5a and sC5b-9 are not increased in atherosclerosis and in established CVD thus suggests that systemic terminal complement pathway activation may be less prominent in mild and stable CVD.

Taken together, the current findings suggest that $\mathrm{C} 5 \mathrm{a}$ and $\mathrm{sC} 5 \mathrm{~b}-9$ play a role in chronic inflammatory processes and in endothelial dysfunction. This is congruent with observations from the orphan diseases, atypical haemolytic uraemic syndrome and paroxysmal nocturnal haemoglobinuria [60, 61]. In those patients, uncontrolled activation of the terminal complement pathway causes systemic inflammation and endothelial damage. A further hallmark of these diseases is venous or microvascular thrombosis, triggered by endothelial damage and coagulation abnormalities. This presentation of vascular damage is markedly different from atherothrombosis in CVD patients of the general population, and suggests that one major point of attack of $\mathrm{C} 5 \mathrm{a}$ and sC5b-9 may be the endothelium of vessel segments other than large arteries. This might explain why we observed an association of $\mathrm{C} 5 \mathrm{a}$ and $\mathrm{sC} \mathrm{Cb}$ 9 with endothelial dysfunction but not with cIMT, AAIx and CVD. The successful use of drugs that inhibit terminal complement activation in the therapy of atypical haemolytic uraemic syndrome and paroxysmal nocturnal haemoglobinuria [62] demonstrates a causal role of terminal complement activation in overt inflammation and endothelial dysfunction, and suggests that the associations of $\mathrm{C} 5 \mathrm{a}$ and $\mathrm{sC} 5 \mathrm{~b}-9$ with endothelial dysfunction and low-grade inflammation observed in the current study may be biologically relevant.

Our study has several strengths but also limitations. A major strength is the availability of both $\mathrm{C} 5 \mathrm{a}$ and $\mathrm{sC} 5 \mathrm{~b}-9$ in a large cohort that has been comprehensively characterized. This allowed us to thoroughly account for potentially confounding factors. A further strength is the availability of several aetiological measures of CVD (CIMT, AAIx, prevalent CVD). The consistent lack of an association of $\mathrm{C} 5 \mathrm{a}$ and sC5b-9 with these measures makes us confident in the robustness of this null finding. We determined three widely used and accepted plasma markers for endothelial dysfunction, but it may be regarded a limitation that we did not have a direct measurement of endothelial (dys)function available. A further limitation of our study is its cross-sectional design, which prohibits conclusions on the direction of these relationships. However in diseased states, complement, inflammation and endothelial function also interact in vicious circles [2], and the present findings suggest that such interrelation also participates in chronic low-grade inflammation and endothelial dysfunction.

In summary, $\mathrm{C} 5 \mathrm{a}$ and sC5b-9, as markers for terminal complement activation, were positively and independently associated with systemic low-grade inflammation and with plasma markers of endothelial dysfunction. This suggests that systemic activation of the terminal complement pathway may participate in a chronic inflammatory state and may impair the vascular endothelium. In contrast, plasma concentrations of $\mathrm{C} 5 \mathrm{a}$ and $\mathrm{sC} 5 \mathrm{~b}-9$ were not associated with markers of atherosclerosis or prevalent CVD. This suggests that in atherosclerosis local generation of $\mathrm{C} 5 \mathrm{a}$ and $\mathrm{sC} 5 \mathrm{~b}-9$ may be more relevant than systemic activation of the terminal complement pathway. Compared to their demonstrated role in acute cardiovascular events, systemic increases in $\mathrm{C5a}$ and sC5b-9 are less prominent in patients with established CVD from the general population. In conclusion, C5a and sC5b-9 
may play a role in chronic inflammation and endothelial dysfunction, but their systemic concentrations are not increased in atherosclerosis and in stable CVD. 


\section{References}

1. Libby $P$, Okamoto $\mathrm{Y}$, Rocha VZ, Folco E. Inflammation in atherosclerosis: transition from theory to practice. Circ. J., 2010. 74: 213-20.

2. Ricklin D, Lambris JD. Complement in immune and inflammatory disorders: pathophysiological mechanisms. J. Immunol., 2013. 190: 3831-8.

3. Ricklin D, Hajishengallis G, Yang K, Lambris JD. Complement: a key system for immune surveillance and homeostasis. Nat. Immunol., 2010. 11: 785-97.

4. Bjerre M, Hansen TK, Flyvbjerg A. Complement activation and cardiovascular disease. Horm. Metab. Res., 2008. 40: 626-34.

5. Ward PA. Functions of C5a receptors. J. Mol. Med., 2009. 87: 375-8.

6. Guo RF, Ward PA. Role of C5a in inflammatory responses. Annu. Rev. Immunol., 2005. 23: 821-52.

7. Kilgore KS, Schmid E, Shanley TP, Flory CM, Maheswari V, Tramontini NL, Cohen H, Ward PA, Friedl HP, Warren JS. Sublytic concentrations of the membrane attack complex of complement induce endothelial interleukin-8 and monocyte chemoattractant protein-1 through nuclear factor-kappa B activation. Am. J. Pathol., 1997. 150: 2019-31.

8. Niculescu F, Badea T, Rus H. Sublytic C5b-9 induces proliferation of human aortic smooth muscle cells: role of mitogen activated protein kinase and phosphatidylinositol 3-kinase. Atherosclerosis, 1999. 142: 47-56.

9. Niculescu F, Soane L, Badea T, Shin M, Rus H. Tyrosine phosphorylation and activation of Janus kinase 1 and STAT3 by sublytic C5b-9 complement complex in aortic endothelial cells. Immunopharmacology, 1999. 42: 187-93.

10. Triantafilou K, Hughes TR, Triantafilou M, Morgan BP. The complement membrane attack complex triggers intracellular Ca2+ fluxes leading to NLRP3 inflammasome activation. J. Cell Sci., 2013. 126: 2903-13.

11. Tegla CA, Cudrici C, Patel S, Trippe R, 3rd, Rus V, Niculescu F, Rus H. Membrane attack by complement: the assembly and biology of terminal complement complexes. Immunol. Res., 2011. 51: 45-60.

12. Okamoto K, Wang W, Jacobs DO, Terai C. Activation of multiple signaling pathways by terminal complement complexes involved in myocellular sodium homeostasis. Tohoku J. Exp. Med., 2004. 202: 113-22.

13. Bossi F, Fischetti F, Pellis V, Bulla R, Ferrero E, Mollnes TE, Regoli D, Tedesco F. Platelet-activating factor and kinin-dependent vascular leakage as a novel functional activity of the soluble terminal complement complex. J. Immunol., 2004. 173: 6921-7.

14. Corallini F, Bossi F, Gonelli A, Tripodo C, Castellino G, Mollnes TE, Tedesco F, Rizzi L, Trotta F, Zauli G, Secchiero $\mathrm{P}$. The soluble terminal complement complex (SC5b-9) up-regulates osteoprotegerin expression and release by endothelial cells: implications in rheumatoid arthritis. Rheumatology, 2009. 48: 293-8.

15. Manthey HD, Thomas AC, Shiels IA, Zernecke A, Woodruff TM, Rolfe B, Taylor SM. Complement C5a inhibition reduces atherosclerosis in ApoE $\%$ mice. FASEB J., 2011. 25: 2447-55.

16. Lewis RD, Jackson CL, Morgan BP, Hughes TR. The membrane attack complex of complement drives the progression of atherosclerosis in apolipoprotein E knockout mice. Mol. Immunol., 2010. 47: 1098-105.

17. Schmiedt W, Kinscherf R, Deigner HP, Kamencic H, Nauen O, Kilo J, Oelert H, Metz J, Bhakdi S. Complement C6 deficiency protects against diet-induced atherosclerosis in rabbits. Arterioscler. Thromb. Vasc. Biol., 1998. 18: $1790-5$.

18. Speidl WS, Exner M, Amighi J, Kastl SP, Zorn G, Maurer G, Wagner O, Huber K, Minar E, Wojta J, Schillinger M. Complement component C5a predicts future cardiovascular events in patients with advanced atherosclerosis. Eur. Heart J., 2005. 26: 2294-9.

19. Speidl WS, Exner M, Amighi J, Mlekusch W, Sabeti S, Kastl SP, Zorn G, Maurer G, Wagner O, Huber K, Minar E, Wojta J, Schillinger M. Complement component C5a predicts restenosis after superficial femoral artery balloon angioplasty. J. Endovasc. Ther., 2007. 14: 62-9.

20. Speidl WS, Katsaros KM, Kastl SP, Zorn G, Huber K, Maurer G, Wojta J, Christ G. Coronary late lumen loss of drug eluting stents is associated with increased serum levels of the complement components C3a and C5a. Atherosclerosis, 2010. 208: 285-9.

21. Mellbin LG, Bjerre M, Thiel S, Hansen TK. Complement activation and prognosis in patients with type 2 diabetes and myocardial infarction: a report from the DIGAMI 2 trial. Diabetes Care, 2012. 35: 911-7.

22. Lindberg S, Pedersen SH, Mogelvang R, Galatius S, Flyvbjerg A, Jensen JS, Bjerre M. Soluble form of membrane attack complex independently predicts mortality and cardiovascular events in patients with ST-elevation myocardial infarction treated with primary percutaneous coronary intervention. Am. Heart J., 2012. 164: 78692.

23. Kruijshoop M, Feskens EJ, Blaak EE, de Bruin TW. Validation of capillary glucose measurements to detect glucose intolerance or type 2 diabetes mellitus in the general population. Clin. Chim. Acta, 2004. 341: 33-40.

24. Potier L, Abi Khalil C, Mohammedi K, Roussel R. Use and utility of ankle brachial index in patients with diabetes. Eur. J. Vasc. Endovasc. Surg., 2011. 41: 110-6. 
25. Morgan BP. Complement membrane attack on nucleated cells: resistance, recovery and non-lethal effects. Biochem. J., 1989. 264: 1-14.

26. Jacobs M, van Greevenbroek MM, van der Kallen CJ, Ferreira I, Blaak EE, Feskens EJ, Jansen EH, Schalkwijk CG, Stehouwer CD. Low-grade inflammation can partly explain the association between the metabolic syndrome and either coronary artery disease or severity of peripheral arterial disease: the CODAM study. Eur. J. Clin. Invest., 2009. 39: 437-44.

27. van Bussel BC, Ferreira I, van de Waarenburg MP, van Greevenbroek MM, van der Kallen CJ, Henry RM, Feskens EJ, Stehouwer CD, Schalkwijk CG. Multiple Inflammatory Biomarker Detection in a Prospective Cohort Study: A Cross-Validation between Well-Established Single-Biomarker Techniques and an Electrochemiluminescense-Based Multi-Array Platform. PLoS ONE, 2013. 8: e58576.

28. Wlazlo N, van Greevenbroek MM, Ferreira I, Jansen EJ, Feskens EJ, van der Kallen CJ, Schalkwijk CG, Bravenboer B, Stehouwer CD. Low-grade inflammation and insulin resistance independently explain substantial parts of the association between body fat and serum C3: the CODAM study. Metabolism, 2012. 61: 1787-96.

29. Stam F, van Guldener C, Schalkwijk CG, ter Wee PM, Donker AJ, Stehouwer CD. Impaired renal function is associated with markers of endothelial dysfunction and increased inflammatory activity. Nephrol. Dial. Transplant., 2003. 18: 892-898.

30. Jacobs M, van Greevenbroek MM, van der Kallen CJ, Ferreira I, Blaak EE, Feskens EJ, Jansen EH, Schalkwijk CG, Stehouwer CD. The association between the metabolic syndrome and peripheral, but not coronary, artery disease is partly mediated by endothelial dysfunction: the CODAM study. Eur. J. Clin. Invest., 2011. 41: 16775.

31. Engelen L, Ferreira I, Gaens KH, Henry RM, Dekker JM, Nijpels G, Heine RJ, t Hart LM, van Greevenbroek MM, van der Kallen CJ, Blaak EE, Feskens EJ, Ten Cate H, Stehouwer CD, Schalkwijk CG. The association between the $-374 \mathrm{~T} / \mathrm{A}$ polymorphism of the receptor for advanced glycation endproducts gene and blood pressure and arterial stiffness is modified by glucose metabolism status: the Hoorn and CoDAM studies. J. Hypertens., 2010. 28: 285-93.

32. Atsma F, Bartelink ML, Grobbee DE, van der Schouw YT. Best reproducibility of the ankle-arm index was calculated using Doppler and dividing highest ankle pressure by highest arm pressure. J. Clin. Epidemiol., 2005. 58: $1282-8$.

33. Alberti KG, Zimmet PZ. Definition, diagnosis and classification of diabetes mellitus and its complications. Part 1: diagnosis and classification of diabetes mellitus provisional report of a WHO consultation. Diabetic Med., 1998. 15: 539-53.

34. van Greevenbroek MM, Jacobs M, van der Kallen CJ, Blaak EE, Jansen EH, Schalkwijk CG, Feskens EJ, Stehouwer $\mathrm{CD}$. Human plasma complement $\mathrm{C} 3$ is independently associated with coronary heart disease, but only in heavy smokers (the CODAM study). Int. J. Cardiol., 2012. 154: 158-62.

35. Wendel-Vos GC, Schuit AJ, Saris WH, Kromhout D. Reproducibility and relative validity of the short questionnaire to assess health-enhancing physical activity. J. Clin. Epidemiol., 2003. 56: 1163-9.

36. Levey AS, Bosch JP, Lewis JB, Greene T, Rogers N, Roth D. A more accurate method to estimate glomerular filtration rate from serum creatinine: a new prediction equation. Modification of Diet in Renal Disease Study Group. Ann. Intern. Med., 1999. 130: 461-70.

37. Friedewald WT, Levy RI, Fredrickson DS. Estimation of the concentration of low-density lipoprotein cholesterol in plasma, without use of the preparative ultracentrifuge. Clin. Chem., 1972. 18: 499-502.

38. van Greevenbroek MM. The expanding role of complement in adipose tissue metabolism and lipoprotein function. Curr. Opin. Lipidol., 2009. 20: 353-4.

39. van Greevenbroek MM, Ghosh S, van der Kallen CJ, Brouwers MC, Schalkwijk CG, Stehouwer CD. Up-regulation of the complement system in subcutaneous adipocytes from nonobese, hypertriglyceridemic subjects is associated with adipocyte insulin resistance. J. Clin. Endocrinol. Metab., 2012. 97: 4742-52.

40. Thewissen MM, Damoiseaux JG, Duijvestijn AM, van Greevenbroek MM, van der Kallen CJ, Feskens EJ, Blaak EE, Schalkwijk CG, Stehouwer CD, Cohen Tervaert JW, Ferreira I. Abdominal fat mass is associated with adaptive immune activation: the CODAM Study. Obesity (Silver Spring), 2011. 19: 1690-8.

41. Preacher KJ, Hayes AF. Asymptotic and resampling strategies for assessing and comparing indirect effects in multiple mediator models. Behav. Res. Methods, 2008. 40: 879-91.

42. Schram MT, Stehouwer CD. Endothelial dysfunction, cellular adhesion molecules and the metabolic syndrome. Horm. Metab. Res., 2005. 37 Suppl 1: 49-55.

43. Chong AY, Blann AD, Lip GY. Assessment of endothelial damage and dysfunction: observations in relation to heart failure. QJM, 2003. 96: 253-67.

44. Cole SR, Platt RW, Schisterman EF, Chu H, Westreich D, Richardson D, Poole C. Illustrating bias due to conditioning on a collider. Int. J. Epidemiol., 2010. 39: 417-20.

45. Ward PA. Role of C5 activation products in sepsis. ScientificWorldJournal, 2010. 10: 2395-402. 
46. Lim J, Iyer A, Suen JY, Seow V, Reid RC, Brown L, Fairlie DP. C5aR and C3aR antagonists each inhibit dietinduced obesity, metabolic dysfunction, and adipocyte and macrophage signaling. FASEB J., 2013. 27: 822-31.

47. Manthey HD, Woodruff TM, Taylor SM, Monk PN. Complement component 5a (C5a). Int. J. Biochem. Cell Biol., 2009. 41: 2114-7.

48. Schieferdecker HL, Schlaf G, Jungermann K, Gotze O. Functions of anaphylatoxin C5a in rat liver: direct and indirect actions on nonparenchymal and parenchymal cells. Int. Immunopharmacol., 2001. 1: 469-81.

49. Lupia E, Del Sorbo L, Bergerone S, Emanuelli G, Camussi G, Montrucchio G. The membrane attack complex of complement contributes to plasmin-induced synthesis of platelet-activating factor by endothelial cells and neutrophils. Immunology, 2003. 109: 557-63.

50. Monsinjon T, Gasque P, Chan P, Ischenko A, Brady JJ, Fontaine MC. Regulation by complement C3a and C5a anaphylatoxins of cytokine production in human umbilical vein endothelial cells. FASEB J., 2003. 17: $1003-14$.

51. Foreman KE, Vaporciyan AA, Bonish BK, Jones ML, Johnson KJ, Glovsky MM, Eddy SM, Ward PA. C5a-induced expression of P-selectin in endothelial cells. J. Clin. Invest., 1994. 94: 1147-55.

52. Tedesco F, Pausa M, Nardon E, Introna M, Mantovani A, Dobrina A. The cytolytically inactive terminal complement complex activates endothelial cells to express adhesion molecules and tissue factor procoagulant activity. J. Exp. Med., 1997. 185: 1619-27.

53. Stehouwer CD. Is measurement of endothelial dysfunction clinically useful? Eur. J. Clin. Invest., 1999. 29: 45961.

54. Oksjoki R, Kovanen PT, Meri S, Pentikainen MO. Function and regulation of the complement system in cardiovascular diseases. Front. Biosci., 2007. 12: 4696-708.

55. Patel S, Thelander EM, Hernandez M, Montenegro J, Hassing H, Burton C, Mundt S, Hermanowski-Vosatka A, Wright SD, Chao YS, Detmers PA. ApoE(-/) mice develop atherosclerosis in the absence of complement component C5. Biochem. Biophys. Res. Commun., 2001. 286: 164-70.

56. Arumugam TV, Magnus T, Woodruff TM, Proctor LM, Shiels IA, Taylor SM. Complement mediators in ischemiareperfusion injury. Clin. Chim. Acta, 2006. 374: 33-45.

57. Szeplaki G, Szegedi R, Hirschberg K, Gombos T, Varga L, Karadi I, Entz L, Szeplaki Z, Garred P, Prohaszka Z, Fust G. Strong complement activation after acute ischemic stroke is associated with unfavorable outcomes. Atherosclerosis, 2009. 204: 315-20.

58. Bjerre M, Kistorp C, Hansen TK, Faber J, Lip GY, Hildebrandt P, Flyvbjerg A. Complement activation, endothelial dysfunction, insulin resistance and chronic heart failure. Scand. Cardiovasc. J., 2010. 44: 260-6.

59. Yasuda M, Takeuchi K, Hiruma M, lida H, Tahara A, Itagane H, Toda I, Akioka K, Teragaki M, Oku H. The complement system in ischemic heart disease. Circulation, 1990. 81: 156-163.

60. Schmidtko J, Peine S, El-Housseini Y, Pascual M, Meier P. Treatment of atypical hemolytic uremic syndrome and thrombotic microangiopathies: a focus on eculizumab. Am. J. Kidney Dis., 2013. 61: 289-99.

61. Hill A, Kelly RJ, Hillmen P. Thrombosis in paroxysmal nocturnal hemoglobinuria. Blood, 2013. 121: 4985-96; quiz 5105.

62. Schrezenmeier H, Hochsmann B. Drugs that inhibit complement. Transfus. Apher. Sci., 2012. 46: 87-92. 


\section{Supplemental data}

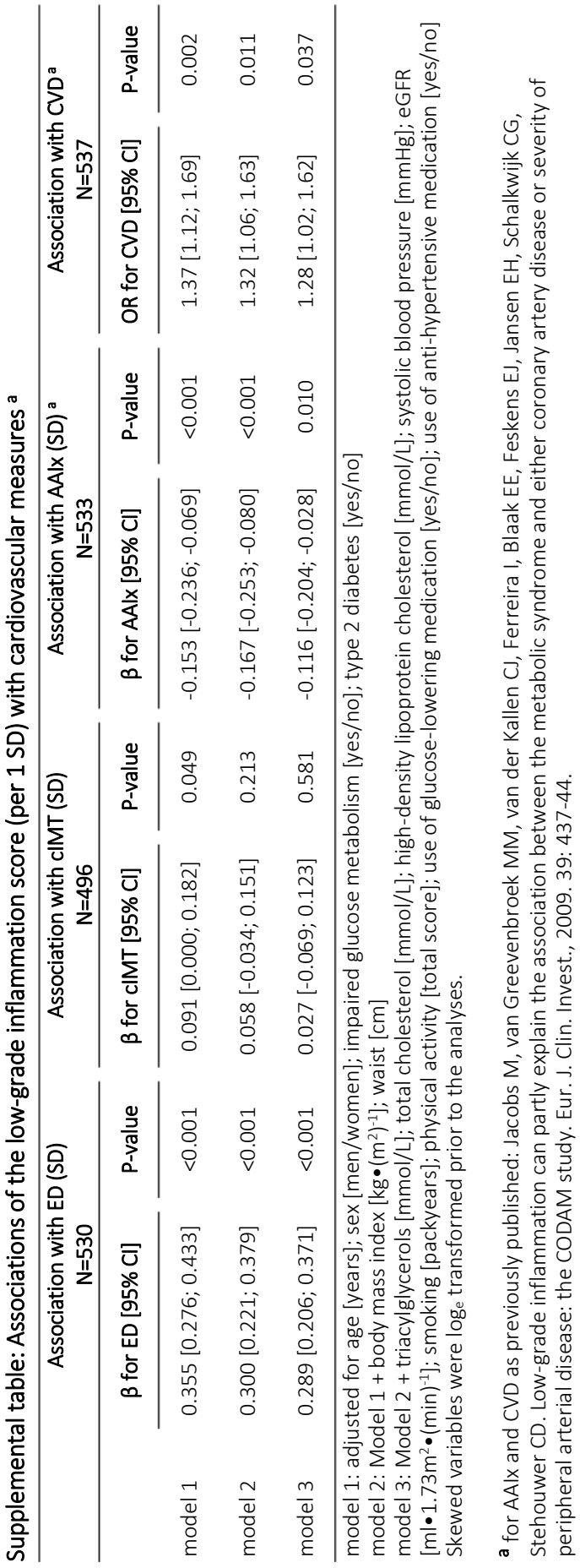




\section{Chapter 6 CHSDfGr 9}

The alternative complement pathway is longitudinally associated with adverse cardiovascular outcomes: The CODAM study

Thrombosis and Haemostasis, 2015. 115 [Epub 8 Oct., doi 10.1160/th15-05-0439]

E. Hertle, I.C.W. Arts, C.J.H. van der Kallen, E.J.M. Feskens, C.G. Schalkwijk,

C.D.A. Stehouwer, M.M.J. van Greevenbroek 


\begin{abstract}
The alternative pathway of complement activation is highly reactive and can be activated spontaneously in the vasculature. Activation may contribute to vascular damage and development of cardiovascular disease (CVD). We aimed to investigate functional components of the alternative pathway in cardiovascular risk.

We studied 573 individuals who were followed-up for seven years. At baseline, we measured the enhancer properdin; the rate-limiting protease factor $D(F D)$; and a marker of systemic activation, $\mathrm{Bb}$. Using generalized estimating equations, we investigated their longitudinal associations with cardiovascular events (CVE, N=89), CVD ( $N=159)$, low-grade inflammation (LGI), endothelial dysfunction (ED) and carotid intima-media thickness (CIMT). Furthermore, we investigated associations with incident CVE $(\mathrm{N}=39)$ and CVD $(\mathrm{N}=73)$ in 342 participants free of CVD at baseline. CVE included myocardial infarction, stroke, cardiac angioplasty and/or cardiac bypass. CVD additionally included ischaemia on an electrocardiogram and/or ankle-brachial index<0.9.

In adjusted analyses, properdin was positively associated with CVE (per 1SD, longitudinal $\mathrm{OR}=1.36$ [1.07; 1.74], OR for incident $\mathrm{CVE}=1.53$ [1.06; 2.20]), but not with CVD. Properdin was also positively associated with $\mathrm{ED}(\beta=0.13[95 \% \mathrm{Cl} 0.06 ; 0.20])$, but not with LGI or CIMT. FD and Bb were positively associated with LGI (per 1SD, FD: $\beta=0.21[0.12 ; 0.29], B b: \beta=0.14$ [0.07; 0.21]), and ED (FD: $\beta=0.20$ [0.11;0.29], Bb: $\beta=0.10$ [0.03; 0.18]), but not with cIMT, CVE or CVD.

Taken together, this suggests that the alternative complement pathway contributes to processes of vascular damage, and that in particular a high potential to enhance alternative pathway activation may promote unfavourable cardiovascular outcomes in humans.
\end{abstract}




\section{Introduction}

The complement system has repeatedly been implicated in cardiovascular disease (CVD) [1-4]. Complement can be activated by the classical, the lectin and the alternative pathway of complement activation, which all converge on the central complement component C3, resulting in activation of the terminal complement pathway [5]. The alternative complement pathway can be activated spontaneously in the circulation and functions also as amplifier of other routes of complement activation $[5,6]$. Alternative pathway factors are highly reactive, and can start a self-amplifying mechanism to promote complement attack and damage [7, 8]. The endothelium, especially, is subjected to constant attack of the alternative pathway $[9,10]$. Complement activation on endothelial cells induces endothelial activation and release of inflammatory mediators [11, 12]. Moreover, systemic complement activation is associated with low-grade inflammation, potentially via effects on liver and adipose tissue [13]. Furthermore, complement is now recognized to participate in coagulation and fibrinolysis $[14,15]$. The alternative complement pathway may thus be instrumental in different processes leading to vascular damage and development of cardiovascular disease (CVD).

As yet, human studies on the alternative pathway factors properdin, factor $D$ (FD) and factor $\mathrm{B}(\mathrm{FB})$ or its activated product $\mathrm{Bb}$ in relation to cardiovascular or metabolic disease are scarce. FD, also known as adipsin, is mainly produced in adipose tissue [16]. FD was shown to increase with obesity and was elevated in individuals with hypertension [17-19]. The only study on FD and CVD reported FD to be associated with risk of ischaemic stroke [18], but not with risk of coronary heart disease (CHD) [19]. FB is produced in the liver, by immune cells, endothelial cells and also in adipose tissue. Expression of FB in adipose tissue was observed to correlate with a worse lipid profile or disturbed glucose metabolism [20]. Its activated product $\mathrm{Bb}$ was, in one case-control study, shown to be elevated in patients with acute myocardial infarction (MI), but not in patients with angina pectoris compared to healthy controls [21]. Properdin is produced by immune and endothelial cells [16, 22] and has so far only been measured in one human cohort, which found properdin to be elevated in individuals with first-degree relatives with type 2 diabetes (T2DM) and in individuals with the metabolic syndrome [23]. These previous human studies thus suggest that the alternative pathway might be involved in cardiometabolic disease. However, there are no prospective studies on $\mathrm{Bb}$ and properdin, and there is no study on CVD that measured several factors of the alternative pathway at the same time.

Therefore, we investigated these different functional components of the alternative pathway in a prospective cohort of middle-aged, Caucasian individuals - the CODAM study. We hypothesized that higher capacity to activate the alternative pathway and higher activation may contribute to vascular damage. We measured properdin, which is the only known positive regulator of complement activation; FD, which is a protease that participates in the alternative pathway activation mechanism; and $\mathrm{Bb}$, a marker of systemic alternative pathway activation (figure 1) [6, 24]. We evaluated their associations with development of cardiovascular events (CVE) and CVD. Furthermore, we explored their relationship with processes underlying the development of CVD, i.e. with biomarker scores reflecting low- 
grade inflammation and endothelial dysfunction and with carotid intima-media thickness (cIMT) as reflection of atherosclerosis.

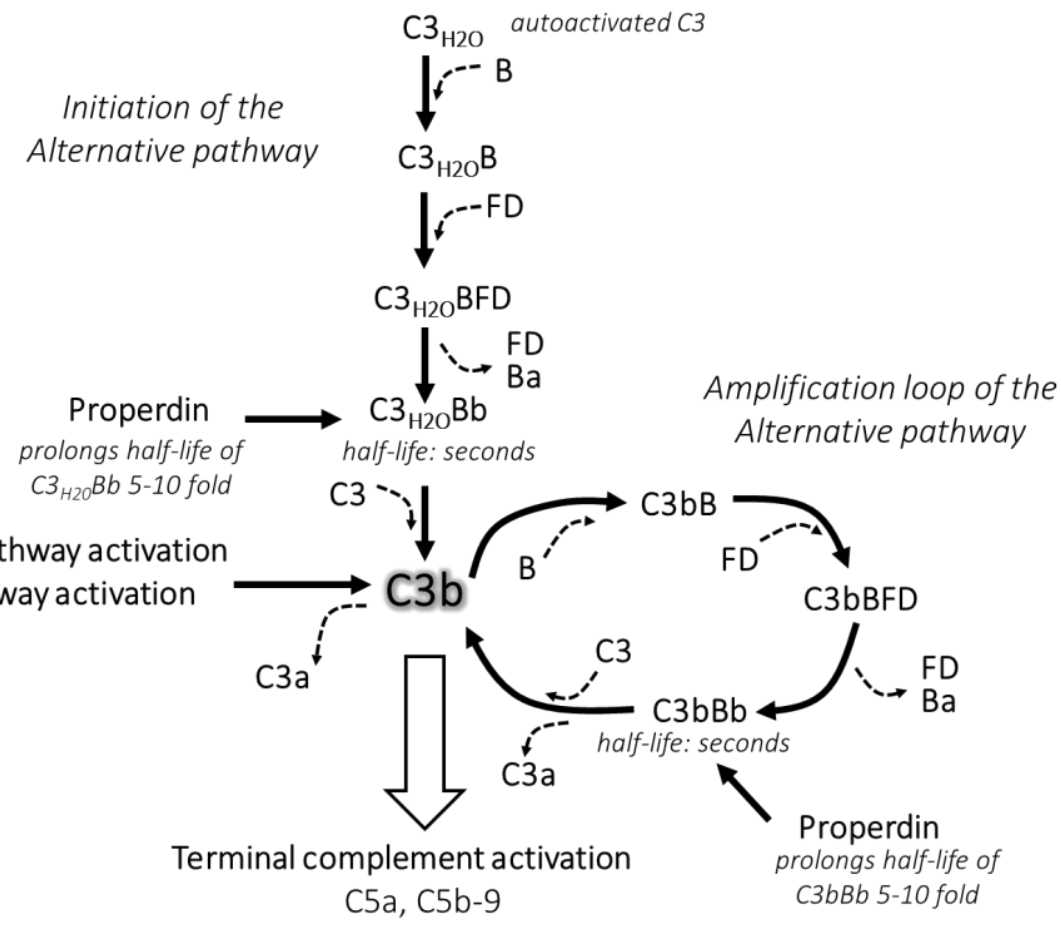

Figure 1: The alternative pathway of complement activation. The alternative pathway is initiated by activation of $\mathrm{C3}$, originating either from $\mathrm{C} 3$ autoactivation $(\mathrm{C} 3 \mathrm{H} 2 \mathrm{O})$ or from $\mathrm{C} 3$ activation as result of previous alternative, classical or lectin pathway activation (C3b). Activated $C 3$ binds Factor $B(B)$, and the complex of activated $C 3$ and $B$ binds factor $D$ (FD). FD is a constitutively active protease and cleaves $\mathrm{B}$ in $\mathrm{Ba}$ and $\mathrm{Bb}$. This complex of activated $\mathrm{C} 3$ and $\mathrm{Bb}$ functions itself as $\mathrm{C} 3$ convertase and activates further $\mathrm{C} 3$ molecules by cleaving $\mathrm{C} 3$ in $\mathrm{C} 3 \mathrm{~b}$ and $\mathrm{C} 3 \mathrm{a}$. Binding of properdin to the $\mathrm{C} 3 \mathrm{H} 2 \mathrm{OBb}$ or $\mathrm{C} 3 \mathrm{bBb}$ complex prolongs the half-life of the active $\mathrm{C} 3$-convertase. Thereby the alternative pathway can expand rapidly and can amplify a few activated $\mathrm{C} 3$ molecules into extensive $\mathrm{C} 3$ activation. Furthermore, the alternative pathway also serves as potent amplification loop of C3 activation initiated via the classical and lectin pathway. Currently, the alternative pathway is estimated to account for $\approx 80 \%$ of complement activation originating from initial classical or lectin pathway activation [6]. 


\section{Materials and methods}

\subsection{Study population}

The Cohort on Diabetes and Atherosclerosis Maastricht (CODAM) includes 574 participants who were selected from a population-based cohort study if they were Caucasian, aged $>40$ years, and had one or more cardiometabolic risk factor (BMl>25 kg/m², use of antihypertensive medication, positive family history of T2DM, history of gestational diabetes and/or glycosuria) [4]. After a median follow-up of 7.0 years [interquartile range 6.9-7.1 years], 495 individuals participated in follow-up measurements. The study was approved by the Medical Ethics Committee of the Maastricht University Medical Centre. All participants gave written informed consent. Participants included in the current analyses are summarized in figure 2.

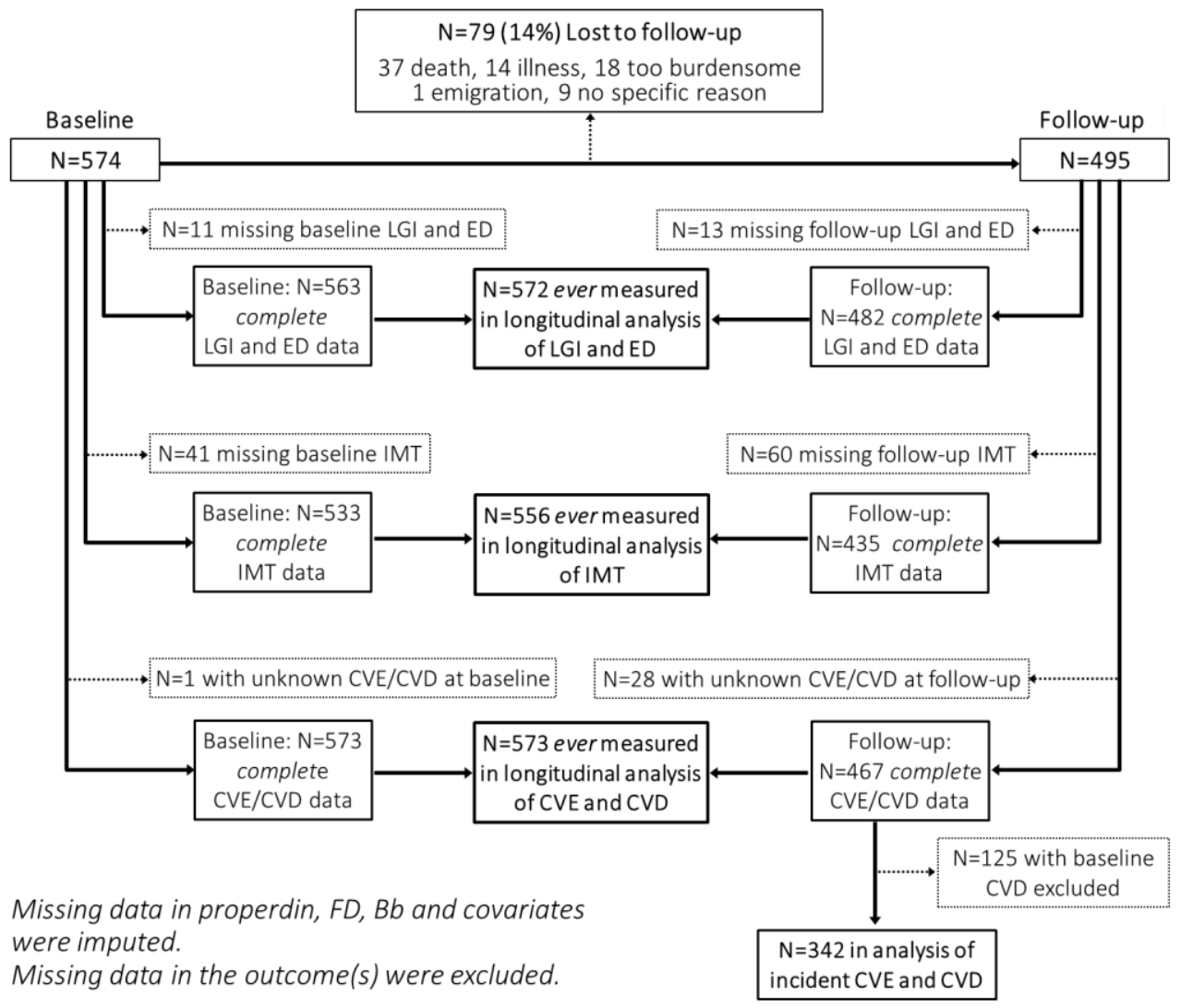

Figure 2: Flow-chart of study participants. CVD, cardiovascular disease; CVE, cardiovascular events; ED, endothelial dysfunction; LGI, low-grade inflammation; cIMT, carotid intima-media thickness. $N=572$ ever measured in longitudinal analysis of $\mathrm{LGI}$ and $\mathrm{ED}$, of which $\mathrm{N}=473$ have complete data at both baseline and follow-up, $\mathrm{N}=90$ have complete data only at baseline and $\mathrm{N}=9$ have complete data only at follow-up, adding up to 1045 observations. $\mathrm{N}=556$ ever measured in longitudinal analysis of cIMT, of which $\mathrm{N}=412$ have complete data at both baseline and follow-up, $\mathrm{N}=121$ have complete data only at baseline and $\mathrm{N}=23$ have complete data only at follow-up, adding up to 968 observations. $\mathrm{N}=573$ ever measured in longitudinal analysis of CVE and CVD, of which $\mathrm{N}=467$ have complete data at both baseline and follow-up, $\mathrm{N}=106$ have complete data only at baseline, adding up to 1040 observations. 


\subsection{Measurements}

Venous blood samples were collected after an overnight fast, processed as described before and stored at $-80^{\circ}[4]$.

Complement factors (measured at baseline): Aliquots were thawed rapidly at $37^{\circ} \mathrm{C}$ and subsequently transferred to ice. Properdin was measured in citrate plasma that had never been thawed before with sandwich-ELISA, with an inter-assay coefficient of variation of $7.7 \%$. For this, microtiter plates were coated overnight at $4{ }^{\circ} \mathrm{C}$ with $1 \mu \mathrm{g} / \mathrm{ml}$ monoclonal mouse anti-human properdin antibody (HYB039-04, BioPorto, Gentofte, Denmark) in carbonate buffer ( $\mathrm{pH}$ 9.6). After 1 wash with PBS containing $0.05 \%$ Tween20 (PBS-Tween) plates were blocked at $20^{\circ} \mathrm{C}$ for $1 \mathrm{~h}$ with $2 \%$ bovine serum albumin (BSA). Plasma and purified properdin (Catalogue number A412, Quidel, San Diego, USA) dilutions were prepared in PBS-Tween with $0.5 \%$ BSA. After 3 washes, plasma samples (diluted 1:500) and properdin standards were incubated at $20{ }^{\circ} \mathrm{C}$ for $2 \mathrm{~h}$. After 3 washes, plates were incubated with $1 \mu \mathrm{g} / \mathrm{ml}$ biotinylated mouse anti-human properdin antibody (HYB039-04-B, BioPorto) for $1 \mathrm{~h}$ at $20^{\circ} \mathrm{C}$. After 4 washes, plates were incubated with streptavidin-horseradish peroxidase for $1 \mathrm{~h}$ at $20^{\circ} \mathrm{C}$. After 4 washes, the amount of bound properdin was determined with a chromogenic reaction using o-phenylenediamine. Absorbance was read at $450 \mathrm{~nm}$ and the amount of properdin in the samples was calculated based on a spline regression curve $(1.5-100 \mathrm{ng} / \mathrm{ml})$. FD was measured in EDTA plasma that had been thawed only once before using a commercially available ELISA development kit (DuoSet, R\&D Systems, Minneapolis, USA) at a $1 / 4000$ dilution according to the manufacturer's instructions, inter-assay variation $4.2 \%$. Bb was measured in EDTA plasma that had never been thawed before by ELISA (MicroVue Bb EIA kit, Quidel, Catalogue number A027, San Diego, USA), inter-assay variation 5.6\%. . At the time these measurements were performed, samples had been stored for $>10$ years. We cannot fully exclude that the absolute concentrations of these factors were to some extent affected during storage, but if this has happened, the extent appears to have been minor. Bb has been reported to be relatively stable even during repeated freeze-thaw cycles [25] and the concentrations of $\mathrm{Bb}$ we observed were exactly within the reference range reported by the manufacturer and by others [26]. Also for properdin and FD, concentrations measured in our study were exactly as reported in pivotal publications in the field [26-28]. Notably, for properdin and FD, absolute concentrations reported in previous studies varied around 4-fold for properdin and 1000-fold for FD [18, 19, 23, 28-30]. C3 was measured twice, once in serum by automatic analyser (Hitachi 912) with a Roche kit (Roche Diagnostics Netherlands BV), inter-assay variation $2.1 \%[4,31]$, and once in plasma by IMMAGE immunochemistry system C3 assay (Beckman Coulter), inter-assay variation 7\%. The two measurements were calibrated after cross-validation as described [32], and the mean of two measurements was used [33].

Biomarkers of inflammation and endothelial dysfunction (measured at baseline and followup): C-reactive protein (CRP), interleukin-6, soluble intercellular adhesion molecule-1 (sICAM-1), serum amyloid A, soluble E-selectin and soluble vascular cell adhesion molecule-1 (sVCAM-1) were measured twice in the baseline samples: once with individual ELISA assays $[34,35]$, and once paired with the follow-up samples using a multiarray detection system 
(MesoScale Discovery, SECTOR Imager 2400). For baseline values, the two measurements were calibrated after cross-validation as described [32], and the mean of two measurements was used. Baseline and follow-up interleukin- 8 and tumour necrosis factor- $\alpha$ were measured paired using a multiarray detection system (MesoScale Discovery, SECTOR Imager 2400, Gaithersburg, Maryland, USA) [32]. Baseline and follow-up von Willebrand factor was measured with an in-house ELISA as described previously [36].

Cardiovascular measures (measured at baseline and follow-up): cIMT was measured at both the left and right common carotid artery 10-20 mm proximal to the carotid bulb with ultrasound (baseline: Ultramark 4+, Advance Technology Laboratories, Bothel, Washington, USA [37]; follow-up: Picus, ESAOTE, Maastricht, Netherlands). CVE were defined as the occurrence of myocardial infarction (MI); coronary bypass; percutaneous coronary intervention and/or stroke reported by questionnaires; as well as signs of $\mathrm{MI}$ on an electrocardiogram (Minnesota codes 1-1 or 1-2). CVD additionally included signs of coronary ischaemia (Minnesota codes 1-3, 4-1, 4-2, 4-3, 5-1, 5-2, 5-3 or 7-1); non-traumatic limb amputation; and/or an ankle-brachial index $<0.9$ [35].

Covariates: Normal glucose metabolism (NGM), impaired glucose metabolism (IGM), T2DM, waist, blood pressure, smoking, habitual physical activity, estimated glomerular filtration rate, HDL-cholesterol, LDL-cholesterol, total cholesterol, triacylglycerols, use of antihypertensive, lipid-modifying and glucose-lowering medication were measured as described $[4,31]$. Use of anti-thrombotic medication was defined as use of either anti-platelet agents or coumarin derivatives.

\subsection{Statistical methods}

Main analyses: We used multiple imputation to impute missing data in the exposure and covariates in order to decrease bias and to increase the power of the analyses [38, 39]. Imputation of missing values has the purpose to avoid the omission of observations with missing data. Especially when observations are not missing completely at random, exclusion of those observations may result in bias in the observed associations. Furthermore, missing values of a single variable can cumulatively result in the exclusion of a substantial part of study population, which leads to a loss of power [39]. We imputed missing values in properdin $(\mathrm{N}=6)$ or $\mathrm{FD}(\mathrm{N}=11)$ and missing values of covariates $(\mathrm{N}=22)$. Missing data in the outcome variables were not imputed (see figure 2 ). The percentage of missing values per variable varied from $0 \%(\mathrm{Bb}$, age, sex NGM, IGM, T2DM, blood pressure, use of medication, lipids) to $2.1 \%$ (smoking pack years). We generated five imputed datasets and the reported results were derived from pooled analyses of all five data sets.

Normally distributed variables are presented as mean \pm standard deviation (SD). Skewed variables are presented as medians (interquartile range). Skewed variables were $\log _{\mathrm{e}}$-transformed to achieve normal distribution prior to the analyses. To provide a robust estimate of inflammation and endothelial dysfunction with minimization of random error, composite scores were calculated of CRP, interleukin-6, interleukin-8, tumour necrosis factor- $\alpha$, sICAM1 and serum amyloid A for inflammation, and of von Willebrand factor, soluble E-selectin, sICAM-1 and SVCAM-1 for endothelial dysfunction [4]. For this, each individual biomarker of 
inflammation or endothelial dysfunction was standardized to the mean of the baseline and follow-up examination (combined Z-score: [individual value minus mean of all values]/SD of all values). These individual Z-scores were averaged into an inflammation score and an endothelial dysfunction score. To allow for a direct comparison of the effect sizes, baseline properdin, FD, Bb and C3 were also converted to their respective Z-scores. Consequently, in all analyses the associations are presented per 1 SD increase in properdin, FD, Bb or C3.

Longitudinal associations of baseline properdin, FD and $\mathrm{Bb}$ with inflammation, endothelial dysfunction and CIMT were investigated using linear GEE. The regression coefficient from GEE indicates the relationship between properdin, FD or Bb (measured at baseline) and the respective outcome (measured at baseline and follow-up) over the 7-year follow-up period. Longitudinal associations with CVE and CVD were investigated with logistic GEE. Logistic GEE includes participants with prevalent and with incident disease. The longitudinal OR obtained in GEE thus combines disease-free participants and participants with either prevalent or incident disease in one measure of association. We used an exchangeable correlation structure and tested whether the association of properdin, FD and Bb with inflammation, endothelial dysfunction, CIMT, CVE or CVD differed over time by including an interaction term with time (baseline / follow-up) in the GEE models. We did not find such interactions (P-value>0.05). All GEE models were adjusted for follow-up time [years]. Logistic regression analysis was used to study the association with incident CVE and CVD in individuals who were free of CVD at baseline. All GEE and logistic regression analyses were adjusted for baseline confounders. First we adjusted for variables that are related to the inclusion procedure, i.e. stratified sampling for age and sex, and oversampling for IGM and T2DM (model 1, age [years]; sex [men/women]; impaired glucose metabolism [yes/no]; type 2 diabetes [yes/no]). Next we adjusted for cardiovascular risk factors and medication use that might affect inflammatory and immunological processes (model 2, waist [cm]; systolic blood pressure $[\mathrm{mmHg}]$; estimated glomerular filtration rate $\left[\mathrm{ml} \bullet 1.73 \mathrm{~m}^{2} \bullet \mathrm{min}^{-1}\right]$; current smoking [yes/no], former smoking [yes/no], amount smoked [pack years], physically active [yes/no]; use of lipid-modifying medication [yes/no]; use of glucose-lowering medication [yes/no]; use of anti-hypertensive medication [yes/no], use of anti-thrombotic medication [yes/no]). In order to explore potential pathways between properdin, FD or Bb and the development of CVE and CVD, we additionally adjusted the logistic models 2 for baseline values of the inflammation score, the endothelial dysfunction score and CIMT, respectively. This was only done if, in linear GEE, a significant association between was observed between properdin, FD or Bb and the inflammation score, the endothelial dysfunction score or CIMT.

Additional analyses: In the main analyses we did not adjust for plasma lipids (triacylglycerols, total cholesterol and HDL-cholesterol) because lipids may act as a confounder but may also lie in the causal path between alternative pathway activation and cardiovascular endpoints. Genetic mouse models suggested that the alternative pathway may causally influence lipid metabolism [40, 41], and human studies suggested involvement in postprandial lipid metabolism $[42,43]$. Adjustment for lipids may therefore result in overadjustment while unadjusted analyses may suffer from residual confounding. In the additional analyses we therefore additionally adjusted model 2 of the main analyses for plasma lipids. Furthermore, 
we repeated all analyses with exclusion of participants with a possible acute inflammation (serum CRP concentrations $>10 \mathrm{mg} / \mathrm{L}, \mathrm{n}=38$ at baseline and $\mathrm{n}=42$ at follow-up). Next, we tested for interaction with sex by including interaction terms with sex to the fully adjusted models. Furthermore, we performed complete case analyses including only participants with complete data in properdin, $\mathrm{FD}, \mathrm{Bb}$ and covariates, resulting in $\mathrm{N}=537$ (982 observations) in the GEE analyses of inflammation and endothelial dysfunction, $\mathrm{N}=519$ (904 observations) in the GEE analyses of CIMT, N=537 (975 observations) in the logistic GEE analyses on CVD and CVE, and $\mathrm{N}=317$ in the analyses on incident CVD and CVE.

Lastly, we evaluated the role of $\mathrm{C} 3$ in significant associations of properdin, FD or Bb with incident CVD and CVE. C3 has been implicated in CVD and processes contributing to CVD in several studies [13] and forms part of the alternative pathway activation mechanism (figure 1). Therefore, we first investigated the association of C3 itself with incident CVD and CVE. Subsequently, we adjusted significant associations of properdin, FD or Bb with incident CVD or CVE for C3.

Statistical analyses were performed using the SPSS package version 22.0 (SPSS, Chicago, IL, USA) and statistical significance was set at $P<0.05$. 
Table 1: Baseline characteristics of the total study population and according to tertiles of properdin concentrations

\begin{tabular}{|c|c|c|c|c|}
\hline & \multirow{2}{*}{$\begin{array}{c}\begin{array}{l}\text { Total study } \\
\text { population }\end{array} \\
\mathrm{N}=573^{*}\end{array}$} & \multicolumn{3}{|c|}{ Tertiles of baseline plasma properdin concentration } \\
\hline & & $\begin{array}{c}1^{\text {st }} \text { tertile } \\
1.69-5.52 \mathrm{mg} / \mathrm{L}\end{array}$ & $\begin{array}{c}\text { 2nd tertile } \\
5.53-6.47 \mathrm{mg} / \mathrm{L}\end{array}$ & $\begin{array}{c}\text { 3rd tertile } \\
6.48-11.21 \mathrm{mg} / \mathrm{L}\end{array}$ \\
\hline Properdin (mg/L) & $6.06 \pm 1.28$ & $4.75 \pm 0.66$ & $6.00 \pm 0.27$ & $7.43 \pm 0.90$ \\
\hline $\mathrm{FD}(\mathrm{mg} / \mathrm{L})$ & $1.00 \pm 0.24$ & $0.96 \pm 0.25$ & $1.03 \pm 0.24$ & $1.01 \pm 0.21$ \\
\hline $\mathrm{Bb}(\mathrm{mg} / \mathrm{L})$ & $0.72 \pm 0.19$ & $0.74 \pm 0.20$ & $0.72 \pm 0.19$ & $0.69 \pm 0.17$ \\
\hline $\mathrm{C} 3(\mathrm{~g} / \mathrm{L})$ & $1.02 \pm 0.16$ & $0.94 \pm 0.15$ & $1.02 \pm 0.14$ & $1.08 \pm 0.16$ \\
\hline Age (years) & $59.6 \pm 7.0$ & $58.8 \pm 7.2$ & $59.9 \pm 6.9$ & $60.1 \pm 6.6$ \\
\hline Sex (\%men) & 61 & 59 & 66 & 58 \\
\hline NGM/IGM/T2DM (\%) & $52 / 22 / 26$ & $61 / 18 / 21$ & $47 / 25 / 28$ & $49 / 23 / 28$ \\
\hline $\mathrm{HbA1c}(\%)$ & $6.0 \pm 0.8$ & $5.9 \pm 0.8$ & $6.0 \pm 0.9$ & $6.0 \pm 0.8$ \\
\hline Waist (cm) & $99 \pm 12$ & $97 \pm 12$ & $99 \pm 12$ & $102 \pm 11$ \\
\hline Systolic blood pressure (mmHg) & $140 \pm 19$ & $137 \pm 19$ & $140 \pm 19$ & $143 \pm 18$ \\
\hline Diastolic blood pressure (mmHg) & $82 \pm 9$ & $80 \pm 9$ & $82 \pm 9$ & $84 \pm 9$ \\
\hline Triacylglycerols (mmol/L) & $1.4[1.0-2.2]$ & $1.10[0.90-1.60]$ & $1.50[1.00-2.00]$ & $1.60[1.20-2.20]$ \\
\hline Total cholesterol (mmol/L) & $5.2 \pm 1.0$ & $5.0 \pm 0.9$ & $5.2 \pm 1.0$ & $5.4 \pm 1.0$ \\
\hline $\mathrm{HDL}(\mathrm{mmol} / \mathrm{L})$ & $1.2 \pm 0.3$ & $1.3 \pm 0.3$ & $1.2 \pm 0.4$ & $1.1 \pm 0.3$ \\
\hline $\mathrm{LDL}(\mathrm{mmol} / \mathrm{L})$ & $3.3 \pm 0.9$ & $3.1 \pm 0.9$ & $3.3 \pm 0.9$ & $3.5 \pm 0.8$ \\
\hline eGFR $\left(\mathrm{ml} / \mathrm{min} / 1.73 \mathrm{~cm}^{2}\right)$ & $94[83-106]$ & 93 [83-103] & 95 [83-105] & $94[84-111]$ \\
\hline Physically active (\%) & 62 & 62 & 67 & 57 \\
\hline Current smokers/former smokers (\%) & $20 / 51$ & $18 / 51$ & $21 / 52$ & $21 / 48$ \\
\hline $\begin{array}{l}\text { Pack years of smoking in former/current } \\
\text { smokers }\end{array}$ & $23[12-36]$ & $24[13-36]$ & 23 [13-39] & 23 [11-37] \\
\hline Use of anti-hypertensive medication (\%) & 38 & 35 & 39 & 41 \\
\hline Use of glucose-lowering medication (\%) & 13 & 14 & 11 & 15 \\
\hline Use of lipid-modifying medication (\%) & 19 & 20 & 18 & 20 \\
\hline Use of anti-thrombotic medication (\%) & 21 & 19 & 22 & 22 \\
\hline $\mathrm{CRP}(\mathrm{mg} / \mathrm{L})$ & $2.07[0.97-3.92]$ & $1.62[0.75-3.16]$ & $2.10[1.02-4.40]$ & $2.31[1.21-4.20]$ \\
\hline Interleukin-6 (ng/L) & $1.55[1.13-2.27]$ & $1.40[1.01-2.29]$ & $1.59[1.17-2.22]$ & $1.62[1.21-2.30]$ \\
\hline Interleukin-8 (ng/L) & $4.40[3.60-5.56]$ & $4.45[3.55-5.56]$ & $4.31[3.59-5.63]$ & $4.44[3.63-5.57]$ \\
\hline Tumour necrosis factor- $\alpha$ (ng/L) & $6.21[5.26-7.52]$ & $5.91[5.01-7.22]$ & $6.38[5.45-7.58]$ & $6.43[5.48-7.87]$ \\
\hline sICAM-1 (mg/L) & $0.21[0.19-0.24]$ & $0.20[0.18-0.23]$ & $0.22[0.19-0.25]$ & $0.22[0.20-0.25]$ \\
\hline Serum amyloid $A$ (mg/L) & $1.42[0.99-2.26]$ & $1.31[0.92-2.21]$ & $1.49[1.00-2.30]$ & $1.51[1.04-2.32]$ \\
\hline $\begin{array}{l}\text { Von Willebrand factor (\% of normal } \\
\text { pooled plasma) }\end{array}$ & 119 [91-161] & $120[87-164]$ & 118 [91-154] & $120[92-164]$ \\
\hline Soluble E-selectin $(\mu \mathrm{g} / \mathrm{L})$ & $8.16[5.98-10.40]$ & $7.62[5.33-10.08]$ & 8.33 [6.47-10.48] & $8.67[6.57-10.62]$ \\
\hline sVCAM-1 ( $\mu \mathrm{g} / \mathrm{L})$ & 332 [293-379] & 316 [285-359] & 334 [299-382] & 347 [297-393] \\
\hline Inflammation score & $-0.09 \pm 0.94$ & $-0.31 \pm 1.00$ & $-0.02 \pm 0.93$ & $0.06 \pm 0.87$ \\
\hline Endothelial dysfunction score & $-0.07 \pm 0.96$ & $-0.34 \pm 0.99$ & $0.03 \pm 0.91$ & $0.10 \pm 0.92$ \\
\hline Carotid IMT ( $\mu \mathrm{m})$ & $774 \pm 157$ & $760 \pm 156$ & $781 \pm 163$ & $780 \pm 153$ \\
\hline Prevalent CVD (\%) & 28 & 29 & 23 & 31 \\
\hline Prevalent CVE (\%) & 16 & 16 & 14 & 16 \\
\hline
\end{tabular}

Data are presented as mean \pm standard deviation (normally distributed variables), median [interquartile range] (skewed variables) or proportion (\%, categorical variables). CRP indicates C-reactive protein; CVD, cardiovascular disease; CVE, cardiovascular events; FD, factor D; eGFR, estimated glomerular filtration rate; HbA1c, glycosylated haemoglobin, HDL, high-density lipoprotein; IGM, impaired glucose metabolism; cIMT, carotid intima-media thickness; LDL, low-density lipoprotein; NGM, normal glucose metabolism; sICAM-1, soluble intercellular adhesion molecule-1; sVCAM-1, soluble vascular cell adhesion molecule-1; T2DM, type 2 diabetes mellitus.

* data were available in $\mathrm{N}=547$ ( $\mathrm{HbA1c}$ ) to 573 participants. 


\section{Results}

Table 1 shows baseline characteristics of the study population ( $N=573$ ) according to tertiles of baseline properdin concentrations. Characteristics according to tertiles of FD and $\mathrm{Bb}$ are given in supplemental table 1.

\subsection{Associations of properdin, FD and $\mathrm{Bb}$ with low-grade inflammation, endothelial dysfunction and cIMT over the 7-year follow-up period}

Participants with follow-up data were marginally younger than the total study population (baseline age $59.1 \pm 7.0$ years compared to $59.6 \pm 7.0$ years) and had a slightly better metabolic profile at baseline (data not shown). Over the 7-year period, participants who were included in the follow-up increased in the inflammation score from $-0.16 \pm 0.90$ to $0.10 \pm 1.05$, in the endothelial dysfunction score from $-0.12 \pm 0.94$ to $0.08 \pm 1.04$, and in cIMT from $768 \pm 157 \mu \mathrm{m}$ to $818 \pm 101 \mu \mathrm{m}$.

Over the 7-year follow-up period, properdin, FD and Bb concentrations were positively and significantly associated with the low-grade inflammation score (table 2, model 1). Upon adjustment for confounders (model 2), the association of properdin with the inflammation score became borderline significant while the associations of $\mathrm{Bb}$ and FD with inflammation were only slightly attenuated. In the fully adjusted model, 1 SD higher baseline properdin, FD or Bb was associated with 0.06 SD [-0.01; 0.12], 0.21 SD [0.12;0.29], and 0.14 SD [0.07; 0.21] higher inflammation score, respectively. Properdin, $\mathrm{FD}$ and $\mathrm{Bb}$ were also

Table 2: Longitudinal associations (GEE) of properdin, FD and $\mathrm{Bb}$ with low-grade inflammation, endothelial dysfunction and cIMT over the 7-year follow-up period

\begin{tabular}{|c|c|c|c|c|c|c|c|c|c|}
\hline & \multicolumn{3}{|c|}{$\begin{array}{l}\text { Low-grade inflammation score } \\
\text { (SD) } \\
\mathrm{N}=572,1045 \text { observations }\end{array}$} & \multicolumn{3}{|c|}{$\begin{array}{l}\text { Endothelial dysfunction score } \\
\text { (SD) } \\
\mathrm{N}=572,1045 \text { observations }\end{array}$} & \multicolumn{3}{|c|}{$\begin{array}{c}\mathrm{clMT} \\
(\mu \mathrm{m}) \\
\mathrm{N}=556,968 \text { observations } \\
\end{array}$} \\
\hline & $\beta$ & $95 \% \mathrm{Cl}$ & P-value & $\beta$ & $95 \% \mathrm{Cl}$ & P-value & $\beta$ & $95 \% \mathrm{Cl}$ & $\mathrm{P}$-value \\
\hline \multicolumn{10}{|c|}{ Properdin (per 1 SD increase) } \\
\hline model 1 & 0.11 & $0.04 ; 0.17$ & 0.001 & 0.17 & $0.10 ; 0.24$ & $<0.001$ & -0.0 & $-10.2 ; 10.2$ & 0.995 \\
\hline model 2 & 0.06 & $-0.01 ; 0.12$ & 0.081 & 0.13 & $0.06 ; 0.20$ & 0.001 & -3.8 & $-13.7 ; 6.1$ & 0.453 \\
\hline \multicolumn{10}{|c|}{ FD (per 1 SD increase) } \\
\hline model 1 & 0.22 & $0.15 ; 0.30$ & $<0.001$ & 0.25 & $0.18 ; 0.33$ & $<0.001$ & -6.2 & $-15.6 ; 3.2$ & 0.197 \\
\hline model 2 & 0.21 & $0.12 ; 0.29$ & $<0.001$ & 0.20 & $0.11 ; 0.29$ & $<0.001$ & -7.0 & $-18.2 ; 4.2$ & 0.220 \\
\hline \multicolumn{10}{|c|}{$\mathrm{Bb}$ (per 1 SD increase) } \\
\hline model 1 & 0.18 & $0.10 ; 0.25$ & $<0.001$ & 0.13 & $0.06 ; 0.20$ & 0.001 & 3.0 & $-6.6 ; 12.6$ & 0.543 \\
\hline model 2 & 0.14 & $0.07 ; 0.21$ & $<0.001$ & 0.10 & $0.03 ; 0.18$ & 0.005 & -2.9 & $-12.4 ; 12.6$ & 0.564 \\
\hline
\end{tabular}

$\beta$ indicates the longitudinal regression coefficient as obtained from GEE which indicates the relationship between baseline properdin, FD and $\mathrm{Bb}$ on the one hand and low-grade inflammation, endothelial dysfunction and cIMT on the other hand over the 7 -year follow-up period; $95 \% \mathrm{Cl}, 95 \%$ confidence interval; FD, factor D; cIMT, carotid intima-media thickness.

model 1: adjusted for age; sex; impaired glucose metabolism; type 2 diabetes, follow-up, follow-up time model 2: model 1 + waist; systolic blood pressure; estimated glomerular filtration rate; current smoking; former smoking; smoking pack years; physical activity; use of lipid-modifying, glucose-lowering, anti-hypertensive or antithrombotic medication 
positively and significantly associated with the endothelial dysfunction score (table 2, model 1). Over the 7-year follow-up period, 1 SD higher baseline properdin, FD or Bb was, in the fully adjusted model (model 2), associated with 0.13 SD [0.06; 0.20], 0.20 SD [0.11; 0.29] and 0.10 SD [0.03; 0.18] higher endothelial dysfunction score, respectively.

In contrast, properdin, FD and $\mathrm{Bb}$ were not associated with cIMT. In model 1, there was no association of properdin, FD or Bb with CIMT (per 1 SD higher properdin: -0.0 $\mu \mathrm{m}$ [-10.2; 10.2]; FD: $-6.2 \mu \mathrm{m}$ [-15.6; 3.2]; and Bb: $3.0 \mu \mathrm{m}[-6.6 ; 12.6])$. Further adjustment did not influence these regression coefficients.

\subsection{Associations of properdin, FD and Bb with CVD and CVE over the 7-year follow-up period}

Next, we investigated associations of baseline properdin, FD and Bb with CVE and CVD in a logistic GEE model (table 3), which includes both prevalent and incident cases.

Properdin was positively and significantly associated with CVE. In model 1, per 1 SD higher properdin the OR for CVE was 1.28 [1.05; 1.55], and further adjustment for confounders did not materially alter this association (model 2, OR was 1.36 [1.07; 1.74]). Properdin was also positively, but borderline significantly, associated with CVD in a model adjusted for age, sex, IGM and T2DM (model 1, per 1 SD higher properdin, the OR for CVD was $1.16[0.98 ; 1.38]$ ). This was however attenuated after adjustment for confounders (OR=1.09 $[0.89 ; 1.33])$. FD was positively and significantly associated with CVE and CVD when adjusted for age, sex, IGM and T2DM (model 1, per 1 SD higher FD, the OR for CVE was 1.25 [1.04; 1.51], and OR for CVD was 1.20 [1.01; 1.42]). These associations were attenuated and became non-significant after adjustment for confounders (model 2). Bb was not clearly associated with CVE or CVD in either model 1 or after full adjustments (model 2).

As shown above, properdin, FD and Bb were positively associated with the inflammation score and/or endothelial dysfunction score. Inflammation and endothelial dysfunction may lie in the causal path leading from alternative pathway activation to cardiovascular endpoints. However, additional adjustment of the association of properdin with CVE for either the endothelial dysfunction score or the inflammation sore in model 2 only slightly attenuated the $\mathrm{OR}$ from 1.36 to $\mathrm{OR}=1.35$ [1.05; 1.73], $\mathrm{P}=0.020$, and $\mathrm{OR}=1.33$ [1.04; 1.70], $\mathrm{P}=0.022$, respectively. Non-significant associations of FD and Bb with CVE or CVD were not materially altered. 
Table 3: Longitudinal associations (logistic GEE) of properdin, FD and Bb with CVE and CVD over the 7-year follow-up period

\begin{tabular}{|c|c|c|c|c|c|c|}
\hline & \multicolumn{3}{|c|}{$\begin{array}{c}\text { CVE } \\
\mathrm{N}=573,1040 \text { observations }\end{array}$} & \multicolumn{3}{|c|}{$\begin{array}{c}\text { CVD } \\
\mathrm{N}=573,1040 \text { observations }\end{array}$} \\
\hline & OR & $95 \% \mathrm{Cl}$ & P-value & OR & $95 \% \mathrm{Cl}$ & P-value \\
\hline \multicolumn{7}{|c|}{ Properdin (per 1 SD increase) } \\
\hline model 1 & 1.28 & $1.05 ; 1.55$ & 0.013 & 1.16 & $0.98 ; 1.38$ & 0.081 \\
\hline model 2 & 1.36 & $1.07 ; 1.74$ & 0.012 & 1.09 & $0.89 ; 1.33$ & 0.398 \\
\hline \multicolumn{7}{|c|}{ FD (per 1 SD increase) } \\
\hline model 1 & 1.25 & $1.04 ; 1.51$ & 0.019 & 1.20 & $1.01 ; 1.42$ & 0.038 \\
\hline model 2 & 1.10 & $0.82 ; 1.46$ & 0.532 & 1.11 & $0.88 ; 1.40$ & 0.371 \\
\hline \multicolumn{7}{|c|}{$\mathrm{Bb}$ (per $1 \mathrm{SD}$ increase) } \\
\hline model 1 & 1.18 & $0.97 ; 1.43$ & 0.100 & 1.11 & $0.94 ; 1.31$ & 0.210 \\
\hline model 2 & 1.15 & $0.90 ; 1.46$ & 0.266 & 1.08 & $0.89 ; 1.33$ & 0.447 \\
\hline
\end{tabular}

OR indicates the longitudinal odds ratio as obtained from logistic GEE which indicates the relationship between baseline properdin, FD and $\mathrm{Bb}$ on the one hand and both prevalent and incident CVE or CVD on the other hand over the 7-year follow-up period; $95 \% \mathrm{Cl}$, 95\% confidence interval; CVD, cardiovascular disease; CVE, cardiovascular events; FD, factor D.

model 1: adjusted for age; sex; impaired glucose metabolism; type 2 diabetes, follow-up, follow-up time model 2: model 1 + waist; systolic blood pressure; estimated glomerular filtration rate; current smoking; former smoking; smoking pack years; physical activity; use of lipid-modifying, glucose-lowering, anti-hypertensive or antithrombotic medication

\subsection{Associations of properdin, FD and Bb with incident CVE and CVD}

The logistic GEE analyses presented above have the advantage that they include more cases; however the OR obtained in GEE does not discriminate between prevalent and incident disease. Theoretically, an OR in GEE may on the one hand represent a cross-sectional association or on the other hand a prospective association, and may in practice be a combination of both. To explore this further, we investigated the associations of baseline properdin, FD and Bb with 7-year risk of CVE and CVD in individuals who were free of CVD at baseline ( $N=342$, table 4). Over the 7-year follow-up period, 73 out of 342 developed CVD (21\%) and 39 of those experienced an actual event (CVE, 11\%). Individuals with incident CVD were at baseline older, more often had T2DM, were characterized by a higher inflammation and endothelial dysfunction score, and a higher cIMT compared to individuals who remained free of CVD during the follow-up (data not shown).

Properdin was positively and significantly associated with incident CVE (table 4). In model 1, per 1 SD higher baseline properdin, the OR for CVE was 1.54 [1.11; 2.14], and further adjustment for confounders did not materially alter this association (model 2, OR was 1.53 [1.06; 2.20]). Properdin was not associated with incident CVD in either model 1 or model 2. FD was positively associated with incident CVE in model 1 (OR was 1.43 [1.03; 1.97], but this association became non-significant after further adjustments. FD was also positively, but non-significantly, associated with incident CVD in model 1 (OR was 1.23 [0.95; 1.61]). This association was attenuated after further adjustments. Bb was not associated with incident 
CVE, and was positively although non-significantly associated with incident CVD in both model 1 and model 2 (model 2, OR=1.25 [0.96; 1.64]).

Additional adjustment of the association of properdin with incident CVE for either the endothelial dysfunction score or the inflammation score in model 2 slightly attenuated the OR from 1.53 to $O R=1.51$ [1.04; 2.18], $P=0.031$ and $O R=1.47$ [1.01; 2.12], $P=0.042$, respectively. Non-significant associations of FD and $\mathrm{Bb}$ with incident CVE or CVD were not materially altered.

Table 4: Associations of properdin, FD and Bb with 7-year incidence of CVE and CVD in participants free of CVD at baseline

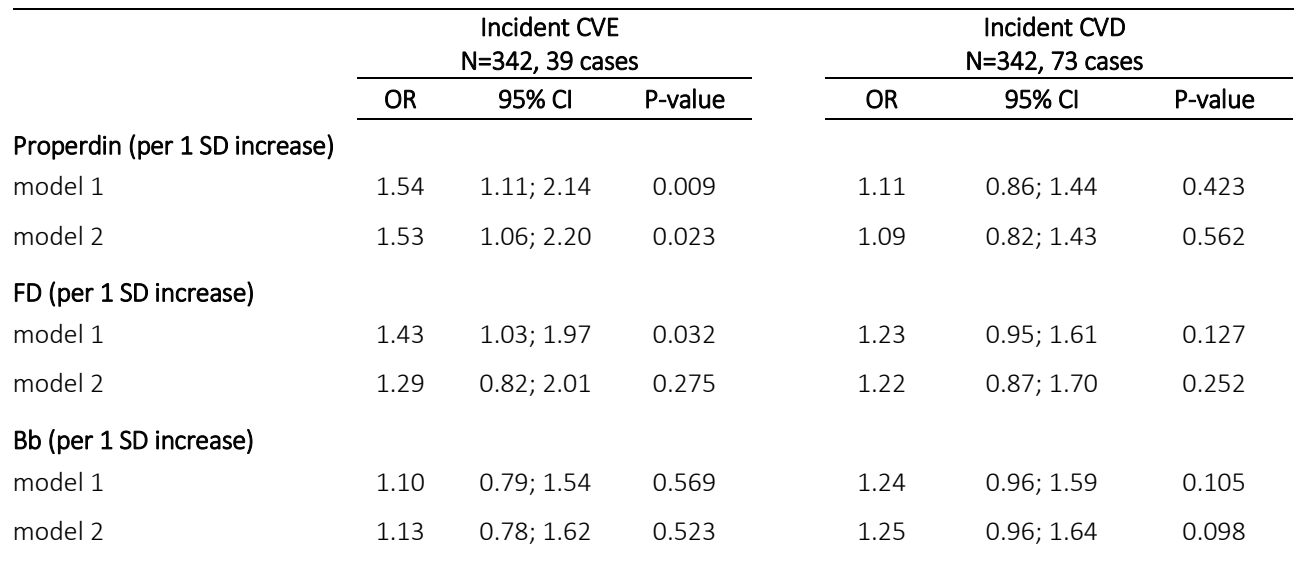

OR indicates odds ratio; 95\% Cl, 95\% confidence interval; CVD, cardiovascular disease; CVE, cardiovascular events; $\mathrm{FD}$, factor $\mathrm{D}$.

model 1: adjusted for age; sex; impaired glucose metabolism; type 2 diabetes

model 2: model 1 + waist; systolic blood pressure; estimated glomerular filtration rate; current smoking; former smoking; smoking pack years; physical activity; use of lipid-modifying, glucose-lowering, anti-hypertensive or antithrombotic medication

\subsection{Additional analyses}

When models 2 in all analyses presented above were additionally adjusted for plasma lipids, the associations of properdin, FD and $\mathrm{Bb}$ with the inflammation score and the endothelial dysfunction score were not materially altered (data not shown). The positive associations of properdin with CVE were also not materially altered. With additional adjustment for lipids, the OR in the logistic GEE was 1.33 [1.04; 1.70], P=0.031, and the OR for incident CVE was 1.46 [0.98; 2.19], $P=0.065$. Exclusion of participants with CRP concentrations above $10 \mathrm{mg} / \mathrm{L}$ did not materially alter the associations (data not shown). No interaction with sex was observed, except for the longitudinal associations of properdin, $\mathrm{FD}$ and $\mathrm{Bb}$ with the endothelial dysfunction score. When stratified for sex, associations of properdin, FD and Bb with the endothelial dysfunction score were more pronounced in women compared to men (supplemental table 2). 
In complete cases analyses, we obtained similar results in all analyses presented above. In 537 participants with complete data, the OR of properdin for CVE in the fully adjusted GEE model was 1.38 [1.08; 1.78], $\mathrm{P}=0.011$ ). In the analysis of incident CVE, the fully adjusted $\mathrm{OR}$ of properdin in 317 participants with complete data was 1.61 [1.09; 2.36], $\mathrm{P}=0.016$ ).

Associations of C3 with incident CVD and CVE, and effect of additional adjustment for C3: In order to relate the association of properdin with CVE to the associations of C3 with CVD or CVE, we first investigated the association of C3 with incident CVD and CVE. In the fully adjusted model, C3 was not associated with incident CVD (per 1 SD, OR was 0.98 [0.69; 1.37], $\mathrm{P}=0.990$ ). The association between C3 and CVE was positive, but was non-significant (OR was $1.19[0.73 ; 1.92], P=0.485)$. When we additionally adjusted the fully adjusted association of properdin with CVE for C3, the association of properdin with CVE was not materially altered (OR was 1.52 [1.04; 2.21], $\mathrm{P}=0.030$ ).

\section{Discussion}

This is the first longitudinal study on the alternative complement pathway in human CVD. We herein performed a comprehensive evaluation of the potential for alternative pathway activation. We measured factors that participate in the activation mechanism - FD and properdin, as well as a systemic estimate of alternative pathway activation - Bb. This study has four main findings: first, properdin was positively associated with endothelial dysfunction and with risk of CVE. Second, FD and Bb were not significantly associated with CVE or CVD, although FD was positively associated with CVE and CVD in analyses adjusted only for age, sex and glucose metabolism status. Third, FD and Bb were positively associated with lowgrade inflammation and endothelial dysfunction. Fourth, properdin, FD and Bb were not associated with cIMT. Taken together, these data implicate the alternative complement pathway in human CVD, and indicate a contribution via inflammatory, endothelial and possibly thrombotic disease mechanisms.

Human data on properdin, FD and Bb in relation to CVD are very scarce. So far, only one study measured properdin, and reported properdin to be elevated in apparently healthy individuals with first-degree relatives with T2DM [23]. In a large cohort of men, FD was associated with risk of ischaemic stroke, but not with risk of CHD $[18,19]$. One former casecontrol study observed $\mathrm{Bb}$ to be elevated in patients upon an acute MI but not in patients with angina pectoris compared to healthy controls [21]. Our current study extends these previous observations by showing that properdin, FD and $\mathrm{Bb}$ were all associated with processes of vascular damage, while in particular properdin was associated with a higher risk of CVE.

Properdin stabilizes the active enzyme complex of the alternative pathway and is the only known positive regulator of complement activation. Moreover, properdin is thought to function not only as stabilizer, but also as activator of the alternative pathway [44]. The alternative pathway is initiated either by random activation of C3 in fluids or on surfaces, or by $\mathrm{C} 3$ activation resulting from classical or lectin pathway activity. Interestingly, properdin was reported to serve as enhancer only upon autonomous activation, but not when the 
alternative pathway was activated secondarily to other pathways [24, 45]. Together with our findings, this suggests that properdin may contribute especially to random (surface) activation of the alternative pathway in the vasculature, which will be most crucially dependent on properdin. In line with this, properdin was in the current study positively associated with endothelial dysfunction, but was not independently associated with lowgrade inflammation. Endothelial dysfunction did however not substantially attenuate the association of properdin with CVE, suggesting that properdin may contribute to CVE through other mechanisms. Notably, properdin was not associated with cIMT. In agreement with this, a recent study in properdin-deficient mice did not report a clear effect on development of atherosclerosis [46]. We emphasize that cIMT reflects thickening of the carotid arterial the wall and that our observations do not preclude that properdin may play a role in other aspects of atherosclerosis, such as in plaque burden or plaque phenotype. We observed that properdin was associated with CVE but only to a lesser extent with CVD. Apparently, properdin was associated particularly with atherothrombotic events, such as stroke and MI, which comprise a major part of the CVE cases while our definition of CVD also includes the less advanced cases such as patients with coronary ischaemia on an electrocardiogram or $A B \mid<0.9$. Participation of properdin in CVE through an effect on thrombosis is indeed supported by recent data that properdin may be involved in platelet activation $[47,48]$.

FD was positively associated with CVE and CVD, but in contrast to what was observed for properdin, these associations were attenuated after full adjustment for confounders. Furthermore, FD was positively and most strongly associated with low-grade inflammation and endothelial dysfunction. FD is a constitutively active protease and is rate-limiting in the activation of the alternative pathway. Human data on FD and CVD are not fully consistent. In a large cohort of men, FD was associated with risk of ischaemic stroke but not with risk of CHD $[18,19]$. In contrast, a plasma proteome study identified FD as risk marker for CHD but not for stroke (ischaemic and haemorrhagic stroke combined) [49]. Our cohort was not powered to distinguish between CHD and stroke, or between ischaemic and haemorrhagic stroke, but we did observe that FD was not associated with cIMT. Altogether, our findings suggest that FD may contribute to the development of CVD through inflammation and endothelial dysfunction. However, the lack of an independent association with CVE or CVD indicates that contribution of FD to CVD risk may be small or dependent on other mechanisms.

We measured $\mathrm{Bb}$ as a marker of systemic alternative pathway activation. Bb was positively associated with inflammation and endothelial dysfunction, but did not show conclusive associations with CVE or CVD. Previous cross-sectional studies did also not show an increase of activated Factor $B$ when patients with angina pectoris or coronary stenosis were compared to healthy controls $[21,50]$. In contrast, Bb was observed to be markedly increased in acute $\mathrm{MI}$ [21], which illustrates that the alternative pathway is indeed activated during CVE. Apparently, systemic levels of $\mathrm{Bb}$ rather reflect dynamic processes such as inflammation and endothelial dysfunction. A single measurement of $\mathrm{Bb}$ may only reflect acute activation of the alternative pathway in the circulation but may insufficiently reflect the degree of alternative pathway activation over time. 
Higher levels of properdin result in a higher capacity to activate the alternative pathway and may therefore represent activation of the alternative pathway in relevant situations. In the development of CVE, the alternative pathway may be activated at a relatively low level compared to pronounced activation during acute events, but may cumulatively contribute to CVE via sustained, potentially local effects on the vasculature. As alternative explanation for the association of properdin with CVE we consider the possibility that the potential to activate the alternative pathway upon acute incidents in the vasculature determines the severity of subsequent vascular damage. The alternative pathway is thought to be involved in platelet activation and is activated during ischaemia-reperfusion injury [51]. This suggests that a higher capacity to activate the alternative pathway upon haemostatic changes or during acute vascular injury may result in a higher degree of platelet activation and in more tissue injury, respectively. Upon higher properdin levels, plaque rupture or vessel stenosis may then more easily escalate into acute ischaemic events.

In order to relate associations of properdin to those of $\mathrm{C3}$, we also investigated $\mathrm{C3}$ in the current evaluation. C3 was not associated with incident CVD, while the association with incident CVE was positive but non-significant. The association of baseline properdin with future CVE is thus substantially stronger than for C3. In our previous cross-sectional study, the association of C3 with prevalent CVD was positive and significant [31]. Furthermore, we had observed that this association was affected by smoking behaviour and that C3 was in particular associated with atherothrombotic events [4, 31]. In the present study, the number of current smokers $(\mathrm{N}=64)$ in participants free of CVD at baseline was too small to investigate effect modification by smoking behaviour. In our view, the difference between the crosssectional and prospective findings may not exclusively be explained by a lack of power. Notably, in cross-sectional studies, C3 was consistently reported to be associated with CVD [13], while the few prospective studies on C3 and CVD had conflicting findings $[1,52,53]$. These studies investigated incident $\mathrm{CHD}, \mathrm{Ml}$ or stroke, which are manifestations of CVD comparable to our definition of CVE. In the first study, a significant association with MI was observed, but this association was only adjusted for age and sex [1]. In a large study in men $(\mathrm{N} \approx 6000), \mathrm{C} 3$ was not independently associated with $\mathrm{Ml}$ and stroke [52]. Only in one study, an independent association of C3 with CHD was observed [53]. Overall, C3 appears to be a reliable marker of prevalent CVD/CVE, while evidence for an association with future CVD/CVE in individuals without prior CVD is less conclusive. The association of properdin with CVE in our study was also independent of C3. This suggests that baseline properdin may be more relevant than $\mathrm{C} 3$ in the development of CVE.

Our study has several strengths but also limitations. Strengths include its prospective nature, the thorough evaluation of different functional components of the alternative complement pathway, and the detailed phenotyping of participants. Limitations include the relatively small number of cases with incident disease, which may have limited the power in the analysis of incident CVD and especially incident CVE. Furthermore, extrapolation of our findings should be done with caution, as our study includes individuals with at least one cardiometabolic risk factor. Notwithstanding, our cohort represents a large part of typical 
Western populations, namely middle-aged to elderly individuals with a moderately increased risk to develop cardiometabolic disease.

In summary, systemic activation of the alternative complement pathway was associated with a proinflammatory state and more endothelial dysfunction. In particular properdin, which is an enhancer of alternative pathway activation, was associated with a higher risk of CVE. This suggests that spontaneous activation of the alternative pathway in the circulation contributes to several aspects of vascular damage (summarized in figure 3 ). In conclusion, the alternative complement pathway may promote unfavourable cardiovascular outcomes in humans.

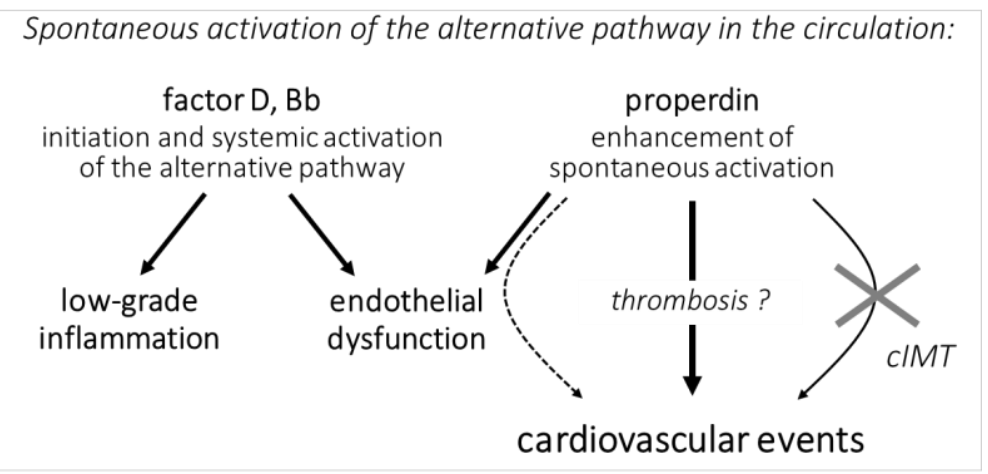

Figure 3: Graphical presentation of main findings. 


\section{References}

1. Muscari A, Bozzoli C, Puddu GM, Sangiorgi Z, Dormi A, Rovinetti C, Descovich GC, Puddu P. Association of serum C3 levels with the risk of myocardial infarction. Am. J. Med., 1995. 98: 357-64.

2. Carter AM. Complement activation: an emerging player in the pathogenesis of cardiovascular disease. Scientifica (Cairo), 2012. 2012: 402783.

3. Hertle E, van Greevenbroek MM, Stehouwer CD. Complement C3: an emerging risk factor in cardiometabolic disease. Diabetologia, 2012. 55: 881-4.

4. Hertle E, van Greevenbroek MM, Arts IC, van der Kallen CJ, Geijselaers SL, Feskens EJ, Jansen EH, Schalkwijk CG, Stehouwer CD. Distinct associations of complement C3a and its precursor C3 with atherosclerosis and cardiovascular disease. The CODAM study. Thromb. Haemost., 2014. 111: 1102-11.

5. Ricklin D, Hajishengallis G, Yang K, Lambris JD. Complement: a key system for immune surveillance and homeostasis. Nat. Immunol., 2010. 11: 785-97.

6. Harboe M, Mollnes TE. The alternative complement pathway revisited. J. Cell. Mol. Med., 2008. 12: $1074-84$.

7. Thurman JM, Holers VM. The central role of the alternative complement pathway in human disease. J. Immunol., 2006. 176: 1305-10.

8. Holers VM. The spectrum of complement alternative pathway-mediated diseases. Immunol. Rev., 2008. 223: 300-16.

9. Fischetti F, Tedesco F. Cross-talk between the complement system and endothelial cells in physiologic conditions and in vascular diseases. Autoimmunity, 2006. 39: 417-28.

10. Zipfel PF, Heinen S, Jozsi M, Skerka C. Complement and diseases: defective alternative pathway control results in kidney and eye diseases. Mol. Immunol., 2006. 43: 97-106.

11. Bossi F, Bulla R, Tedesco F. Endothelial cells are a target of both complement and kinin system. Int. Immunopharmacol., 2008. 8: 143-147.

12. Kerr H, Richards A. Complement-mediated injury and protection of endothelium: lessons from atypical haemolytic uraemic syndrome. Immunobiology, 2012. 217: 195-203.

13. Hertle E, Stehouwer CD, van Greevenbroek MM. The complement system in human cardiometabolic disease. Mol. Immunol., 2014. 61: 135-48.

14. Oikonomopoulou K, Ricklin D, Ward PA, Lambris JD. Interactions between coagulation and complement--their role in inflammation. Semin. Immunopathol., 2012. 34: 151-65.

15. Lupu F, Keshari RS, Lambris JD, Coggeshall KM. Crosstalk between the coagulation and complement systems in sepsis. Thromb. Res., 2014. 133 Suppl 1: S28-31.

16. Morgan BP, Gasque P. Extrahepatic complement biosynthesis: where, when and why? Clin. Exp. Immunol., 1997. 107: 1-7.

17. Mavri A, Stegnar M, Krebs M, Sentocnik JT, Geiger M, Binder BR. Impact of adipose tissue on plasma plasminogen activator inhibitor-1 in dieting obese women. Arterioscler. Thromb. Vasc. Biol., 1999. 19: 15827.

18. Prugger C, Luc G, Haas B, Arveiler D, Machez E, Ferrieres J, Ruidavets JB, Bingham A, Montaye M, Amouyel P, Yarnell J, Kee F, Ducimetiere P, Empana JP. Adipocytokines and the risk of ischemic stroke: the PRIME Study. Ann. Neurol., 2012. 71: 478-86.

19. Luc G, Empana JP, Morange P, Juhan-Vague I, Arveiler D, Ferrieres J, Amouyel P, Evans A, Kee F, Bingham A, Machez E, Ducimetiere P. Adipocytokines and the risk of coronary heart disease in healthy middle aged men: the PRIME Study. Int. J. Obes. (Lond.), 2010. 34: 118-26.

20. Moreno-Navarrete JM, Martinez-Barricarte R, Catalan V, Sabater M, Gomez-Ambrosi J, Ortega FJ, Ricart W, Bluher M, Fruhbeck G, Rodriguez de Cordoba S, Fernandez-Real JM. Complement factor H is expressed in adipose tissue in association with insulin resistance. Diabetes, 2010. 59: 200-9.

21. Yasuda M, Takeuchi K, Hiruma M, lida H, Tahara A, Itagane H, Toda I, Akioka K, Teragaki M, Oku H. The complement system in ischemic heart disease. Circulation, 1990. 81: 156-163.

22. Bongrazio M, Pries AR, Zakrzewicz A. The endothelium as physiological source of properdin: role of wall shear stress. Mol. Immunol., 2003. 39: 669-75.

23. Somani R, Richardson VR, Standeven KF, Grant PJ, Carter AM. Elevated properdin and enhanced complement activation in first-degree relatives of South Asian subjects with type 2 diabetes. Diabetes Care, 2012. 35: 894899.

24. Lesher AM, Nilsson B, Song WC. Properdin in complement activation and tissue injury. Mol. Immunol., 2013. 56: 191-198.

25. Bergseth G, Ludviksen JK, Kirschfink M, Giclas PC, Nilsson B, Mollnes TE. An international serum standard for application in assays to detect human complement activation products. Mol. Immunol., 2013. 56: 232-9. 
26. Oppermann M, Baumgarten H, Brandt E, Gottsleben W, Kurts C, Gotze O. Quantitation of components of the alternative pathway of complement (APC) by enzyme-linked immunosorbent assays. J. Immunol. Methods, 1990. 133: 181-90.

27. Pangburn MK. Analysis of the natural polymeric forms of human properdin and their functions in complement activation. J. Immunol., 1989. 142: 202-7.

28. Schwaeble WJ, Reid KB. Does properdin crosslink the cellular and the humoral immune response? Immunol. Today, 1999. 20: 17-21.

29. Pauly D, Nagel BM, Reinders J, Killian T, Wulf M, Ackermann S, Ehrenstein B, Zipfel PF, Skerka C, Weber BH. A novel antibody against human properdin inhibits the alternative complement system and specifically detects properdin from blood samples. PLoS ONE, 2014. 9: e96371.

30. Amato MC, Pizzolanti G, Torregrossa V, Misiano G, Milano S, Giordano C. Visceral Adiposity Index (VAI) IS Predictive of an Altered Adipokine Profile in Patients with Type 2 Diabetes. PLoS ONE, 2014. 9: e91969.

31. van Greevenbroek MM, Jacobs M, van der Kallen CJ, Blaak EE, Jansen EH, Schalkwijk CG, Feskens EJ, Stehouwer $\mathrm{CD}$. Human plasma complement C3 is independently associated with coronary heart disease, but only in heavy smokers (the CODAM study). Int. J. Cardiol., 2012. 154: 158-62.

32. van Bussel BC, Ferreira I, van de Waarenburg MP, van Greevenbroek MM, van der Kallen CJ, Henry RM, Feskens EJ, Stehouwer CD, Schalkwijk CG. Multiple Inflammatory Biomarker Detection in a Prospective Cohort Study: A Cross-Validation between Well-Established Single-Biomarker Techniques and an Electrochemiluminescense-Based Multi-Array Platform. PLoS ONE, 2013. 8: e58576.

33. Wlazlo N, van Greevenbroek MM, Ferreira I, Feskens EJ, van der Kallen CJ, Schalkwijk CG, Bravenboer B, Stehouwer CD. Complement factor 3 is associated with insulin resistance and with incident type 2 diabetes over a 7-year follow-up period: the CODAM Study. Diabetes Care, 2014. 37: 1900-9.

34. Jacobs M, van Greevenbroek MM, van der Kallen CJ, Ferreira I, Blaak EE, Feskens EJ, Jansen EH, Schalkwijk CG, Stehouwer CD. Low-grade inflammation can partly explain the association between the metabolic syndrome and either coronary artery disease or severity of peripheral arterial disease: the CODAM study. Eur. J. Clin. Invest., 2009. 39: 437-44.

35. Jacobs M, van Greevenbroek MM, van der Kallen CJ, Ferreira I, Blaak EE, Feskens EJ, Jansen EH, Schalkwijk CG, Stehouwer CD. The association between the metabolic syndrome and peripheral, but not coronary, artery disease is partly mediated by endothelial dysfunction: the CODAM study. Eur. J. Clin. Invest., 2011. 41: 16775.

36. Stam F, van Guldener C, Schalkwijk CG, ter Wee PM, Donker AJ, Stehouwer CD. Impaired renal function is associated with markers of endothelial dysfunction and increased inflammatory activity. Nephrol. Dial. Transplant., 2003. 18: 892-898.

37. Engelen L, Ferreira I, Gaens KH, Henry RM, Dekker JM, Nijpels G, Heine RJ, t Hart LM, van Greevenbroek MM, van der Kallen CJ, Blaak EE, Feskens EJ, Ten Cate H, Stehouwer CD, Schalkwijk CG. The association between the -374T/A polymorphism of the receptor for advanced glycation endproducts gene and blood pressure and arterial stiffness is modified by glucose metabolism status: the Hoorn and CoDAM studies. J. Hypertens., 2010. 28: 285-93.

38. Sterne JA, White IR, Carlin JB, Spratt M, Royston P, Kenward MG, Wood AM, Carpenter JR. Multiple imputation for missing data in epidemiological and clinical research: potential and pitfalls. Brit. Med. J., 2009. 338: b2393.

39. Janssen KJ, Donders AR, Harrell FE, Jr., Vergouwe Y, Chen Q, Grobbee DE, Moons KG. Missing covariate data in medical research: to impute is better than to ignore. J. Clin. Epidemiol., 2010. 63: 721-7.

40. Gauvreau D, Roy C, Tom FQ, Lu H, Miegueu P, Richard D, Song WC, Stover C, Cianflone K. A new effector of lipid metabolism: complement factor properdin. Mol. Immunol., 2012. 51: 73-81.

41. Paglialunga S, Fisette A, Yan Y, Deshaies Y, Brouillette JF, Pekna M, Cianflone K. Acylation-stimulating protein deficiency and altered adipose tissue in alternative complement pathway knockout mice. Am. J. Physiol. Endocrinol. Metab., 2008. 294: E521-529.

42. Peake PW, Kriketos AD, Campbell LV, Charlesworth JA. Response of the alternative complement pathway to an oral fat load in first-degree relatives of subjects with type II diabetes. Int. J. Obes. (Lond.), 2005. 29: 42935.

43. Westerink J, Hajer GR, Kranendonk ME, Schipper HS, Monajemi H, Kalkhoven E, Visseren FL. An oral mixed fat load is followed by a modest anti-inflammatory adipocytokine response in overweight patients with metabolic syndrome. Lipids, 2014. 49: 247-54.

44. Cortes C, Ohtola JA, Saggu G, Ferreira VP. Local release of properdin in the cellular microenvironment: role in pattern recognition and amplification of the alternative pathway of complement. Front. Immunol., 2012. 3: 412.

45. Kimura Y, Miwa T, Zhou L, Song WC. Activator-specific requirement of properdin in the initiation and amplification of the alternative pathway complement. Blood, 2008. 111: 732-40.

46. Steiner T, Francescut L, Byrne S, Hughes T, Jayanthi A, Guschina I, Harwood J, Cianflone K, Stover C, Francis S. Protective role for properdin in progression of experimental murine atherosclerosis. PLoS ONE, 2014. 9: e92404. 
47. Ruef J, Kuehnl P, Meinertz T, Merten M. The complement factor properdin induces formation of plateletleukocyte aggregates via leukocyte activation. Platelets, 2008. 19: 359-64.

48. Saggu G, Cortes C, Emch HN, Ramirez G, Worth RG, Ferreira VP. Identification of a novel mode of complement activation on stimulated platelets mediated by properdin and C3(H2O). J. Immunol., 2013. 190: 6457-67.

49. Prentice RL, Zhao S, Johnson M, Aragaki A, Hsia J, Jackson RD, Rossouw JE, Manson JE, Hanash SM. Proteomic risk markers for coronary heart disease and stroke: validation and mediation of randomized trial hormone therapy effects on these diseases. Genome Med., 2013. 5: 112.

50. Horvath Z, Csuka D, Vargova K, Kovacs A, Molnar AA, Gulacsi-Bardos P, Lee S, Varga L, Kiss RG, Preda I, Fust G. Elevated $\mathrm{C} 1 \mathrm{rC} 1 \mathrm{sC} 1 \mathrm{inh}$ levels independently predict atherosclerotic coronary heart disease. Mol. Immunol., 2013. 54: 8-13.

51. Gorsuch WB, Chrysanthou E, Schwaeble WJ, Stahl GL. The complement system in ischemia-reperfusion injuries. Immunobiology, 2012. 217: 1026-33.

52. Engstrom G, Hedblad B, Janzon L, Lindgarde F. Complement C3 and C4 in plasma and incidence of myocardial infarction and stroke: a population-based cohort study. Eur. J. Cardiovasc. Prev. Rehabil., 2007. 14: 392-7.

53. Onat A, Hergenc G, Can G, Kaya Z, Yuksel H. Serum complement C3: a determinant of cardiometabolic risk, additive to the metabolic syndrome, in middle-aged population. Metabolism, 2010. 59: 628-34. 


\section{Supplemental data}

Supplemental table 1: Characteristics of the study population across tertiles of FD and Bb concentrations

a) Tertiles of baseline plasma Factor D concentration

\begin{tabular}{|c|c|c|c|}
\hline & $\begin{array}{c}1 \mathrm{st} \text { tertile } \\
0.45-0.88 \mathrm{mg} / \mathrm{L}\end{array}$ & $\begin{array}{c}\text { 2nd tertile } \\
0.88-1.07 \mathrm{mg} / \mathrm{L}\end{array}$ & $\begin{array}{c}3 \text { rd tertile } \\
1.07-2.16 \mathrm{mg} / \mathrm{L}\end{array}$ \\
\hline Properdin (mg/L) & $5.86 \pm 1.28$ & $6.10 \pm 1.31$ & $6.18 \pm 1.23$ \\
\hline $\mathrm{FD}(\mathrm{mg} / \mathrm{L})$ & $0.77 \pm 0.09$ & $0.97 \pm 0.05$ & $1.26 \pm 0.19$ \\
\hline $\mathrm{Bb}(\mathrm{mg} / \mathrm{L})$ & $0.69 \pm 0.18$ & $0.69 \pm 0.17$ & $0.77 \pm 0.20$ \\
\hline Age (years) & $57.2 \pm 7.3$ & $59.9 \pm 6.6$ & $61.8 \pm 6.3$ \\
\hline Sex (\%men) & 55 & 65 & 65 \\
\hline NGM/IGM/T2DM (\%) & $47 / 26 / 27$ & $55 / 19 / 26$ & $53 / 23 / 24$ \\
\hline $\mathrm{HbA} 1 \mathrm{c}(\%)$ & $6.0 \pm 0.9$ & $6.0 \pm 0.9$ & $6.0 \pm 0.7$ \\
\hline Waist $(\mathrm{cm})$ & $96 \pm 12$ & $99 \pm 11$ & $102 \pm 12$ \\
\hline Systolic blood pressure (mmHg) & $138 \pm 19$ & $142 \pm 18$ & $142 \pm 20$ \\
\hline Diastolic blood pressure $(\mathrm{mmHg})$ & $91 \pm 10$ & $82 \pm 9$ & $82 \pm 9$ \\
\hline Triacylglycerols (mmol/L) & $1.40[0.90-1.80]$ & $1.40[1.00-2.00]$ & $1.50[1.10-2.00]$ \\
\hline Total cholesterol (mmol/L) & $5.2 \pm 1.0$ & $5.2 \pm 1.0$ & $5.2 \pm 1.0$ \\
\hline $\mathrm{HDL}(\mathrm{mmol} / \mathrm{L})$ & $1.3 \pm 0.4$ & $1.2 \pm 0.3$ & $1.1 \pm 0.3$ \\
\hline $\mathrm{LDL}(\mathrm{mmol} / \mathrm{L})$ & $3.3 \pm 0.9$ & $3.3 \pm 0.8$ & $3.3 \pm 0.9$ \\
\hline eGFR $\left(\mathrm{ml} / \mathrm{min} / 1.73 \mathrm{~cm}^{2}\right)$ & $102[91-114]$ & $96[85-104]$ & 86 [76-96] \\
\hline Physically active (\%) & 57 & 70 & 62 \\
\hline Current smokers/former smokers (\%) & $24 / 47$ & $18 / 54$ & 19/51 \\
\hline Pack years of smoking in former/current smokers & $20[11-34]$ & $23[13-36]$ & $26[15-40]$ \\
\hline Use of anti-hypertensive medication (\%) & 29 & 37 & 49 \\
\hline Use of glucose-lowering medication (\%) & 14 & 13 & 12 \\
\hline Use of lipid-modifying medication (\%) & 15 & 20 & 23 \\
\hline Use of anti-thrombotic medication (\%) & 17 & 20 & 26 \\
\hline $\mathrm{CRP}(\mathrm{mg} / \mathrm{L})$ & $1.85[0.89-3.75]$ & $1.74[0.86-3.46]$ & $2.35[1.1-4.46]$ \\
\hline Interleukin-6 (ng/L) & $1.43[0.99-2.09]$ & $1.52[1.15-2.16]$ & $1.80[1.29-2.58]$ \\
\hline Interleukin-8 (ng/L) & $4.09[3.34-5.41]$ & $4.46[3.57-5.92]$ & $4.52[3.82-5.48]$ \\
\hline Tumour necrosis factor- $\alpha$ (ng/L) & $5.65[4.71-6.88]$ & $6.29[5.30-7.35]$ & $6.97[5.79-8.10]$ \\
\hline sICAM-1 (mg/L) & $0.21[0.18-0.23]$ & $0.21[0.19-0.24]$ & $0.22[0.20-0.25]$ \\
\hline Serum amyloid $A(\mathrm{mg} / \mathrm{L})$ & $1.40[0.95-2.20]$ & $1.32[0.96-2.32]$ & $1.61[1.03-2.45]$ \\
\hline Von Willebrand factor (\% of normal pooled plasma) & $110[80-144]$ & 116 [88-163] & $132[97-176]$ \\
\hline Soluble E-selectin $(\mu \mathrm{g} / \mathrm{L})$ & $8.09[5.53-10.52]$ & $8.16[6.40-10.27]$ & $8.20[6.46-10.52]$ \\
\hline sVCAM-1 ( $\mu \mathrm{g} / \mathrm{L})$ & 314 [277-347] & $334[294-378]$ & 356 [308-409] \\
\hline Inflammation score & $-0.30 \pm 0.96$ & $-0.12 \pm 0.95$ & $0.16 \pm 0.88$ \\
\hline Endothelial dysfunction score & $-0.36 \pm 0.92$ & $-0.07 \pm 0.96$ & $0.24 \pm 0.91$ \\
\hline Carotid IMT ( $\mu \mathrm{m})$ & $758 \pm 158$ & $197 \pm 164$ & $774 \pm 145$ \\
\hline Prevalent CVD (\%) & 21 & 29 & 34 \\
\hline Prevalent CVE (\%) & 11 & 17 & 20 \\
\hline
\end{tabular}

Data are presented as mean \pm standard deviation (normally distributed variables), median [interquartile range] (skewed variables) or proportion (\%, categorical variables). CRP indicates C-reactive protein; CVD, cardiovascular disease; CVE, cardiovascular events; FD, Factor D; eGFR, estimated glomerular filtration rate; HbA1c, glycosylated haemoglobin, HDL, high-density lipoprotein; IGM, impaired glucose metabolism; cIMT, carotid intima-media thickness; LDL, low-density lipoprotein; NGM, normal glucose metabolism; sICAM-1, soluble intercellular adhesion molecule-1; sVCAM-1, soluble vascular cell adhesion molecule-1; T2DM, type 2 diabetes mellitus. 


\begin{tabular}{|c|c|c|c|}
\hline & $\begin{array}{c}\text { 1st tertile } \\
0.29-0.63 \mathrm{mg} / \mathrm{L}\end{array}$ & $\begin{array}{c}\text { 2nd tertile } \\
0.63-0.77 \mathrm{mg} / \mathrm{L}\end{array}$ & $\begin{array}{c}\text { 3rd tertile } \\
0.78-1.48 \mathrm{mg} / \mathrm{L}\end{array}$ \\
\hline Properdin (mg/L) & $6.22 \pm 1.32$ & $6.06 \pm 1.16$ & $5.90 \pm 1.33$ \\
\hline $\mathrm{FD}(\mathrm{mg} / \mathrm{L})$ & $0.93 \pm 0.19$ & $1.01 \pm 0.23$ & $1.06 \pm 0.27$ \\
\hline $\mathrm{Bb}(\mathrm{mg} / \mathrm{L})$ & $0.53 \pm 0.07$ & $0.69 \pm 0.04$ & $0.93 \pm 0.14$ \\
\hline Age (years) & $59.8 \pm 7.0$ & $59.6 \pm 6.8$ & $59.4 \pm 7.1$ \\
\hline Sex (\%men) & 65 & 60 & 59 \\
\hline NGM/IGM/T2DM (\%) & $50 / 21 / 30$ & $56 / 23 / 21$ & $53 / 22 / 25$ \\
\hline $\mathrm{HbA} 1 \mathrm{c}(\%)$ & $6.0 \pm 1.0$ & $6.0 \pm 0.7$ & $5.9 \pm 0.7$ \\
\hline Waist $(\mathrm{cm})$ & $100 \pm 11$ & $100 \pm 13$ & $98 \pm 12$ \\
\hline Systolic blood pressure (mmHg) & $141 \pm 19$ & $140 \pm 19$ & $140 \pm 19$ \\
\hline Diastolic blood pressure (mmHg) & $83 \pm 9$ & $82 \pm 9$ & $81 \pm 9$ \\
\hline Triacylglycerols (mmol/L) & $1.40[1.08-2.10]$ & $1.40[1.00-1.90]$ & $1.40[0.90-1.80]$ \\
\hline Total cholesterol (mmol/L) & $5.3 \pm 0.9$ & $5.3 \pm 1.0$ & $5.1 \pm 1.0$ \\
\hline $\mathrm{HDL}(\mathrm{mmol} / \mathrm{L})$ & $1.2 \pm 0.4$ & $1.2 \pm 0.3$ & $1.2 \pm 0.3$ \\
\hline $\mathrm{LDL}(\mathrm{mmol} / \mathrm{L})$ & $3.3 \pm 0.8$ & $3.4 \pm 0.8$ & $3.3 \pm 0.9$ \\
\hline eGFR (ml/min/1.73cm2) & 94 [85-105] & 95 [84-108] & 92 [81-104] \\
\hline Physically active (\%) & 67 & 61 & 59 \\
\hline Current smokers/former smokers (\%) & $9 / 56$ & $26 / 44$ & $26 / 52$ \\
\hline Pack years of smoking in former/current smokers & 19 [9-35] & $24[12-35]$ & 23 [15-39] \\
\hline Use of anti-hypertensive medication (\%) & 34 & 40 & 41 \\
\hline Use of glucose-lowering medication (\%) & 17 & 11 & 12 \\
\hline Use of lipid-modifying medication (\%) & 17 & 19 & 20 \\
\hline Use of anti-thrombotic medication (\%) & 18 & 22 & 22 \\
\hline $\mathrm{CRP}(\mathrm{mg} / \mathrm{L})$ & $1.43[0.80-2.71]$ & $2.32[1.07-4.21]$ & $2.33[1.10-5.15]$ \\
\hline Interleukin-6 (ng/L) & $1.39[1.04-1.94]$ & $1.56[1.13-2.36]$ & $1.73[1.24-2.48]$ \\
\hline Interleukin-8 (ng/L) & $4.25[3.49-5.63]$ & $4.34[3.64-5.19]$ & $4.59[3.77-5.75]$ \\
\hline Tumour necrosis factor- $\alpha$ (ng/L) & $5.95[4.93-7.27]$ & $6.14[5.30-7.52]$ & $6.44[5.53-7.87]$ \\
\hline sICAM-1 (mg/L) & $0.20[0.18-0.23]$ & $0.21[0.19-0.24]$ & $0.23[0.20-0.25]$ \\
\hline Serum amyloid $A(\mathrm{mg} / \mathrm{L})$ & $1.29[0.95-1.91]$ & $1.50[1.06-2.22]$ & $1.62[0.97-2.64]$ \\
\hline Von Willebrand factor (\% of normal pooled plasma) & 118 [87-159] & 119 [93-152] & $122[87-171]$ \\
\hline Soluble E-selectin $(\mu \mathrm{g} / \mathrm{L})$ & $8.63[5.95-10.63]$ & $7.84[5.86-10.13]$ & $8.26[6.53-10.47]$ \\
\hline sVCAM-1 $(\mu \mathrm{g} / \mathrm{L})$ & 331 [283-379] & $324[292-368]$ & $336[302-388]$ \\
\hline Inflammation score & $-0.36 \pm 0.86$ & $-0.07 \pm 0.92$ & $0.15 \pm 0.98$ \\
\hline Endothelial dysfunction score & $-0.18 \pm 0.93$ & $-0.11 \pm 0.93$ & $0.08 \pm 1.00$ \\
\hline Carotid IMT ( $\mu \mathrm{m})$ & $788 \pm 167$ & $759 \pm 156$ & $776 \pm 147$ \\
\hline Prevalent CVD (\%) & 25 & 27 & 31 \\
\hline Prevalent CVE (\%) & 12 & 16 & 19 \\
\hline
\end{tabular}

Data are presented as mean \pm standard deviation (normally distributed variables), median [interquartile range] (skewed variables) or proportion (\%, categorical variables). CRP indicates C-reactive protein; CVD, cardiovascular disease; CVE, cardiovascular events; FD, Factor D; eGFR, estimated glomerular filtration rate; HbA1c, glycosylated haemoglobin, HDL, high-density lipoprotein; IGM, impaired glucose metabolism; cIMT, carotid intima-media thickness; LDL, low-density lipoprotein; NGM, normal glucose metabolism; sICAM-1, soluble intercellular adhesion molecule-1; sVCAM-1, soluble vascular cell adhesion molecule-1; T2DM, type 2 diabetes mellitus. 
Supplemental table 2: Longitudinal associations (GEE) of properdin, $\mathrm{FD}$ and $\mathrm{Bb}$ with endothelial dysfunction over the 7-year follow-up period, stratified according to sex

\begin{tabular}{|c|c|c|c|c|c|c|}
\hline & \multicolumn{3}{|c|}{$\begin{array}{c}\text { Men } \\
\text { Endothelial dysfunction score } \\
\mathrm{N}=350,643 \text { observations }\end{array}$} & \multicolumn{3}{|c|}{$\begin{array}{c}\text { Women } \\
\text { Endothelial dysfunction score } \\
\mathrm{N}=222,402 \text { observations }\end{array}$} \\
\hline & $\beta$ & $95 \% \mathrm{Cl}$ & P-value & $\beta$ & $95 \% \mathrm{Cl}$ & P-value \\
\hline \multicolumn{7}{|c|}{ Properdin (per 1 SD increase) } \\
\hline model 1 & 0.12 & $0.03 ; 0.22$ & 0.011 & 0.24 & $0.14 ; 0.34$ & $<0.001$ \\
\hline model 2 & 0.09 & $-0.01 ; 0.19$ & 0.064 & 0.18 & $0.07 ; 0.29$ & $<0.001$ \\
\hline \multicolumn{7}{|c|}{ FD (per 1 SD increase) } \\
\hline model 1 & 0.19 & $0.08 ; 0.30$ & 0.001 & 0.33 & $0.24 ; 0.43$ & $<0.001$ \\
\hline model 2 & 0.11 & $-0.02 ; 0.23$ & 0.092 & 0.35 & $0.22 ; 0.47$ & $<0.001$ \\
\hline \multicolumn{7}{|c|}{$\mathrm{Bb}$ (per 1 SD increase) } \\
\hline model 1 & 0.11 & $0.01 ; 0.20$ & 0.027 & 0.17 & $0.05 ; 0.29$ & 0.006 \\
\hline model 2: & 0.05 & $-0.04 ; 0.15$ & 0.282 & 0.21 & $0.10 ; 0.32$ & $<0.001$ \\
\hline
\end{tabular}

$\beta$ indicates the longitudinal regression coefficient as obtained from GEE which indicates the relationship between baseline properdin, $\mathrm{FD}$ and $\mathrm{Bb}$ and endothelial dysfunction over the 7-year follow-up period; $95 \% \mathrm{Cl}, 95 \%$ confidence interval; FD, Factor D.

model 1: adjusted for age; sex; impaired glucose metabolism; type 2 diabetes, follow-up time model 2: model 1 + waist; systolic blood pressure; estimated glomerular filtration rate; current smoking; former smoking; smoking pack years; physical activity; use of lipid-modifying, glucose-lowering, anti-hypertensive or antithrombotic medication 


\section{Chapter 7 Cugisfr 1}

Longitudinal associations of factors of the classical complement pathway with cardiovascular outcomes: The CODAM study

E. Hertle, I.C.W. Arts, C.J.H. van der Kallen, E.J.M. Feskens, C.G. Schalkwijk, C.D.A. Stehouwer, M.M.J. van Greevenbroek 


\begin{abstract}
The classical complement pathway has been assigned both protective and pathological effects in cardiovascular disease (CVD). We measured crucial factors of classical pathway activation in a prospective human cohort and determined their associations with incident CVD, with carotid intima-media thickness (cIMT), low-grade inflammation (LGI) and endothelial dysfunction (ED).

We measured systemic concentrations of the pattern recognition factor $\mathrm{C} 1 \mathrm{q}$; the inhibitor $\mathrm{C} 1-\mathrm{INH}$; and the activation marker C4d at baseline in the CODAM study ( $\mathrm{N}=574,61 \%$ men, age $60 \pm 7$ years). Associations with 7-year incidence of CVD (73 cases) were studied with logistic regression analyses. Associations with cIMT and with biomarker scores reflecting LGI and ED, over the 7-year follow-up period, were studied with generalized estimating equations. All analyses were adjusted for potential confounders.

Individuals in the second tertile (T2) of C1q had the lowest incidence of CVD (T1 compared to $\mathrm{T} 2, \mathrm{OR}=2.38$ [95\% Cl: 1.14; 4.95]; T3 compared to T2, OR=1.96 [0.94; 4.07]). C1-INH was not associated with CVD. We observed significant effect modification by sex in associations of C4d with CVD. C4d was inversely associated with CVD in men (per 1SD, OR=0.59 [0.40; $0.87]$ ), while effect estimates were unstable in women. $\mathrm{C} 1 \mathrm{q}, \mathrm{C} 1-\mathrm{INH}$ and $\mathrm{C} 4 \mathrm{~d}$ were not, or inconsistently, associated with LGI, ED and cIMT.

In conclusion, our results suggest a U-shaped association between C1q and CVD. Additionally, C4d may be protective in men. Our findings support the concept that the classical pathway may have both protective and pathological effects in human CVD.
\end{abstract}




\section{Introduction}

The complement system is involved in cardiovascular disease (CVD) and can be activated via different pathways [1]. The classical pathway of complement activation is thought to have both protective and pathological effects in CVD [2-4]. The classical pathway is initiated by pattern recognition of external as well as internal danger signals, such as apoptotic cells, immune-complexes, CRP-marked surfaces, modified lipoproteins and cholesterol crystals (figure 1) [5-7]. Experimental studies suggested that this pattern recognition-induced complement activation can lead to 'silent' removal of unwanted products without full complement activation [4, 8, 9]. However, full activation of the classical pathway can be amplified via the alternative pathway and can induce terminal complement activation and proinflammatory signalling $[10,11]$. In mice models, a dual role of the classical pathway in atherosclerosis was observed with both inhibition and stimulation of classical pathway activation contributing to atherosclerosis: mice genetically deficient in classical pathway components developed more atherosclerosis, apparently because they could not efficiently dispose of apoptotic cells $[12,13]$. On the other hand, pharmacologic inhibition of classical pathway activation in atherosclerosis-prone mice resulted in reduced inflammation and plaque formation [14]. Thus, limited activation of the classical complement pathway may hamper the 'silent' clear-up processes that are considered beneficial for vascular integrity, while extensive activation may contribute to tissue damage and vascular dysfunction. The classical pathway has also been implicated in additional processes underlying CVD, such as in shear stress-induced endothelial dysfunction or in metabolic impairments $[15,16]$.

Human data on the classical pathway in CVD are scarce. Some smaller case-control studies are available, which each measured a single factor of the classical pathway, such as the pattern recognition factor $\mathrm{C} 1 \mathrm{q}$, the inhibitor $\mathrm{C} 1$-Inhibitor (C1-INH) or the activation product C4d (figure 1). These previous studies compared systemic concentrations of these complement factors between patients with CVD and healthy controls, or between patients with stable and unstable angina pectoris. In these studies, no associations were observed between C1q or C4d and CVD, and inconsistent associations were observed for C1-INH [1721]. However, these comparisons may not have provided a conclusive picture, because of their size, their design and the fact that $\mathrm{C} 1 \mathrm{q}, \mathrm{C} 1-\mathrm{INH}$ or $\mathrm{C} 4 \mathrm{~d}$ were measured in established disease. Since the classical pathway may have heterogeneous effects in different stages of CVD, these cross-sectional studies were unsuitable to thoroughly address the role of the classical pathway in the development of CVD

For these reasons, we measured, in a prospective cohort, C1q, C1-INH and C4d, the latter as estimate of classical pathway activation. We investigated associations of C1q, C1-INH and C4d with incident CVD and cardiovascular events (CVE) over a 7-year follow-up period. Furthermore, we aimed to distinguish different processes underlying the aetiology of CVD. For this, we determined associations of $\mathrm{C} 1 \mathrm{q}, \mathrm{C} 1-\mathrm{INH}$ and $\mathrm{C} 4 \mathrm{~d}$ with biomarker scores reflecting low-grade inflammation and endothelial dysfunction, and with cIMT reflecting atherosclerosis. Based on experimental studies that suggested both protective and pathological effects, we hypothesized that both under- and overactivation may lead to more CVD. Because this may manifest itself in non-linear, potentially U-shaped associations with 
cardiovascular outcomes, we carefully checked for potential deviations from linearity in all analyses. Furthermore, we aimed to investigate whether associations are comparable in men and women, given the known sex differences in development and presentation of CVD.

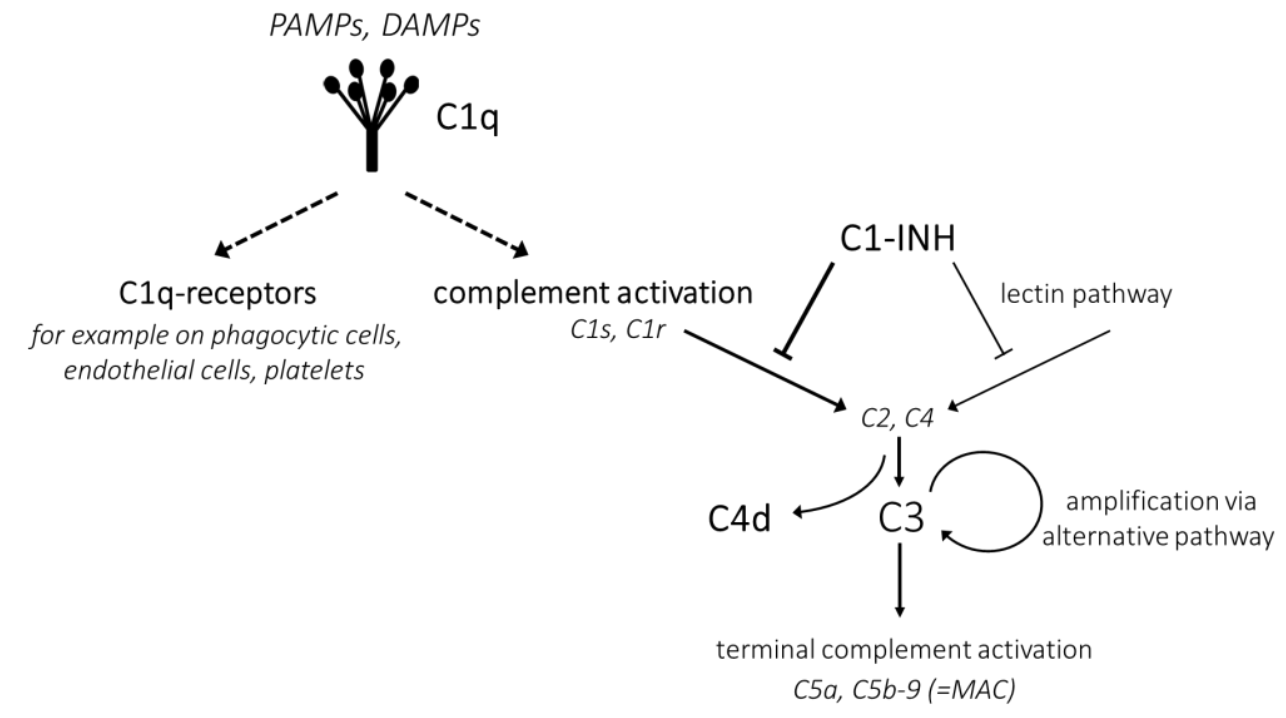

Figure 1: The classical pathway of complement activation. The classical pathway of complement activation is initiated by binding of the pattern recognition factor $\mathrm{Clq}$ to molecular danger signals. C1q recognizes pathogen-associated molecular patterns (PAMPs) on microbes as well as endogenous danger-associated molecular patterns (DAMPs), such as structures exposed on apoptotic cells, immunoglobulin- or CRP-marked surfaces, modified lipoproteins or cholesterol crystals. C1q bound to PAMPs or DAMPs can activate cells that express C1q-receptors, such as immune cells and in particular phagocytic cells. Thereby, C1q can stimulate clear-up processes independently of further complement activation [8]. Besides, binding of $\mathrm{C} 1 \mathrm{q}$ can induce activation of the classical complement cascade. In this cascade, binding of $\mathrm{C} 1 \mathrm{q}$ to surfaces activates the complement proteases $\mathrm{C} 1 \mathrm{r}$ and $\mathrm{C} 1 \mathrm{~s}$, and $\mathrm{C} 1 \mathrm{~s}$ subsequently activates the proteases $\mathrm{C} 2$ and $\mathrm{C} 4$. This cascade is tightly regulated by $\mathrm{C} 1-\mathrm{INH}$, which inhibits the proteases $\mathrm{C} 1 \mathrm{r}$ and $\mathrm{C} 1 \mathrm{~s}$ and thereby blocks activation downstream of $\mathrm{C} 1 \mathrm{q}$ [22]. When the cascade progresses to activation of C4, a C3-convertase is formed, containing activated C4. At this level, C3 activation resulting from initial classical pathway activation can be amplified via the alternative complement pathway, and the common end-route of terminal pathway activation downstream of $\mathrm{C} 3$ can be initiated. Activated C4 that is part of the active $\mathrm{C} 3$ convertase is later degraded, and the fragment $\mathrm{C} 4 \mathrm{~d}$ is released. $\mathrm{C} 4 \mathrm{~d}$ reflects thus progression of classical pathway activation until the common end route starting at $\mathrm{C} 3$. 


\section{Methods}

\subsection{Study design and participants}

The Cohort on Diabetes and Atherosclerosis Maastricht (CODAM) is a prospective cohort study [23]. Briefly, participants were selected from a population-based cohort study if they were Caucasian, aged $>40$ years, and met at least one of the following criteria: BMl>25 kg/m², use of anti-hypertensive medication, a positive family history of type 2 diabetes, history of gestational diabetes and/or glycosuria [24]. 574 participants were included and extensively phenotyped. After a median follow-up of 7.0 years [interquartile range (IQR) 6.9-7.1 years], 495 individuals participated in follow-up measurements (for details see flow-chart, figure 2). The study was approved by the Medical Ethics Committee of the Maastricht University Medical Centre. All participants gave written informed consent.

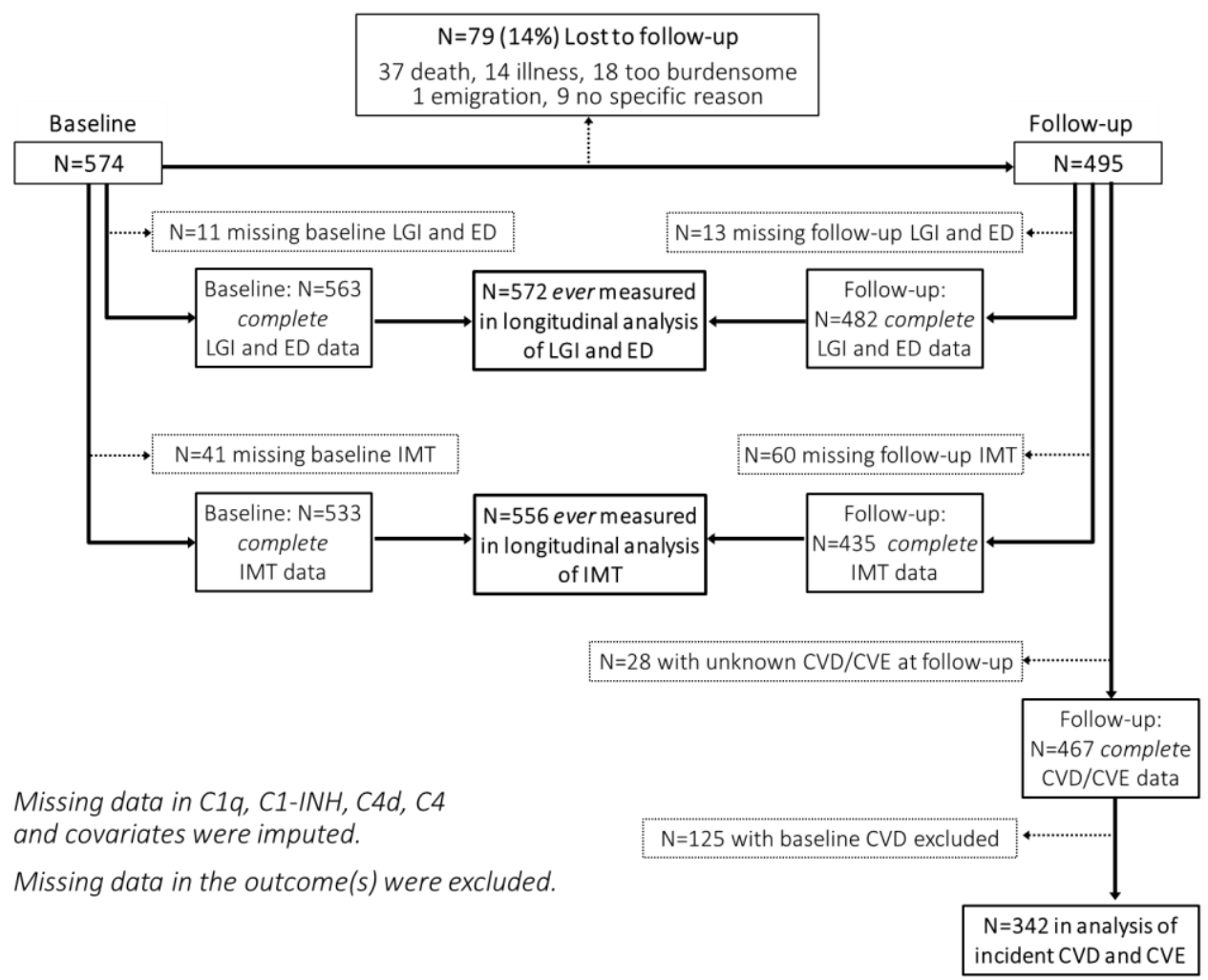

Figure 2: Flow-chart of study participants. CVD, cardiovascular disease; CVE, cardiovascular events; LGI, low-grade inflammation; ED, endothelial dysfunction; IMT, intima-media thickness. $\mathrm{N}=572$ ever measured in longitudinal analysis of $L G I$ and $E D$, of which $N=473$ have complete data at both baseline and follow-up, $N=90$ have complete data only at baseline and $\mathrm{N}=9$ have complete data only at follow-up, adding up to 1045 observations included in the GEE analysis of LGI and ED. $\mathrm{N}=556$ ever measured in longitudinal analysis of IMT, of which $\mathrm{N}=412$ have complete data at both baseline and follow-up, $\mathrm{N}=121$ have complete data only at baseline and $\mathrm{N}=23$ have complete data only at follow-up, adding up to 968 observations included in the GEE analysis of IMT. For the analyses with incident CVE and CVD, persons with prevalent CVD at baseline were excluded. 


\subsection{Measurements}

Participants were asked to stop lipid-modifying medication 14 days prior to blood withdrawal, while other medication was stopped one day beforehand. Venous blood samples were collected by venipuncture after an overnight fast.

Complement factors (baseline only): Aliquots were thawed rapidly at $37^{\circ} \mathrm{C}$ and subsequently transferred to ice. Complement C1q, C1-INH and C4d were measured in EDTA plasma by ELISA (C1q: as described in [25]; C1-INH: MicroVue C1-INH EIA kit, Quidel, Catalogue number A037, San Diego, USA; C4d: MicroVue C4d ElA kit, Quidel, Catalogue number A008, San Diego, USA). C4 was measured as a covariate in serum by auto analyser (Hitachi 912) using a Roche kit assay (Roche Diagnostics Nederland BV, Almere, The Netherlands). Inter-assay coefficients of variation were $18.5 \%$ for $\mathrm{C} 1 \mathrm{q}, 4.4 \%$ for $\mathrm{C} 1-\mathrm{INH}, 4.5 \%$ for $\mathrm{C} 4 \mathrm{~d}$ and $2.0 \%$ for $\mathrm{C} 4$. We cannot fully exclude that the absolute concentrations of these factors were to some extent affected during storage, but if this has happened, the extent was probably minor: $\mathrm{C} 4 \mathrm{~d}$ has been reported to be relatively stable even during repeated freeze-thaw cycles [26]. Concentrations of $\mathrm{C} 1 \mathrm{q}$ and $\mathrm{C} 1-\mathrm{INH}$ in our study were of the same order of magnitude as reported in previous studies $[17,18,21,27,28]$, and concentrations of C4 were within the published reference range [29].

Biomarkers of inflammation and endothelial dysfunction (baseline and follow-up): C-reactive protein (CRP), interleukin-6 (IL-6), soluble intercellular adhesion molecule-1 (sICAM-1), serum amyloid A (SAA), soluble E-selectin (SEs) and soluble vascular cell adhesion molecule1 (sVCAM-1) were measured twice in the baseline samples: once with individual ELISA assays in serum or EDTA plasma [30,31], and once paired with the follow-up samples plasma using a multiarray detection system (MesoScale Discovery, SECTOR Imager 2400, Gaithersburg, Maryland, USA). The two baseline measurements of CRP, IL-6, SICAM-1, SAA, sEs and SVCAM1 were calibrated after cross-validation as described [32], and the mean of two measurements was used for analyses. Paired measurements of baseline and follow-up interleukin-8 (IL-8) and tumour necrosis factor- $\alpha$ (TNF $\alpha$ ) were done in EDTA plasma using a multiarray detection system (MesoScale Discovery, SECTOR Imager 2400) [32]. Baseline and follow-up von Willebrand factor (VWF) was measured with an in-house ELISA in citrated plasma as described [33], and the concentration was expressed as percentage of normal pooled plasma.

Cardiovascular measures: Carotid IMT was measured by a trained vascular sonographer at both the left and right common carotid artery 10-20 mm proximal to the carotid bulb with a non-invasive ultrasound imaging device (at baseline with Ultramark 4+, Advance Technology Laboratories, Bothel, Washington, USA [34]; at follow-up with Picus, ESAOTE, Maastricht, Netherlands). CVD was defined as the occurrence of at least one of the following: previous myocardial infarction (MI); coronary bypass; percutaneous coronary intervention ( $\mathrm{PCl})$ reported by questionnaires (subjects' disease history questionnaire); signs on a 12-lead electrocardiogram of MI (Minnesota codes 1-1 or 1-2) or ischaemia (Minnesota codes 1-3, 41, 4-2, 4-3, 5-1, 5-2, 5-3 or 7-1); stroke or transient ischaemic attack reported by questionnaire; non-traumatic limb amputation; and/or an ankle-brachial index $<0.9$ [35]. The ankle-brachial index was determined as previously described $[31,36]$. This CVD definition 
was established to obtain a reference population that was truly free of CVD. This may, however, have resulted in identification of a heterogeneous group of cases, ranging from subclinical disease (e.g. stable atherosclerosis) to clinical events (atherothrombosis). Therefore we also investigated cardiovascular events (CVE), which comprised MI, stroke, coronary bypass and/or $\mathrm{PCl}$.

Other covariates: Normal glucose metabolism (NGM), impaired glucose metabolism (IGM, combining impaired fasting glucose [IFG] and impaired glucose tolerance [IGT]) and T2DM were identified with a standard 75g OGTT according to the WHO criteria of 1999 [37]. Waist and blood pressure were measured as reported previously [38]. Smoking, habitual physical activity, use of anti-hypertensive, glucose-lowering and lipid-modifying medication was assessed with on-site administered questionnaires [38, 39]. Use of anti-thrombotic medication was defined as use of either anti-platelet agents or coumarin derivatives. Participants were defined as physically active if they were compliant with the Dutch physical activity guideline [40]. Estimated glomerular filtration rate (eGFR) was calculated with the MDRD formula [41] using creatinine levels, measured in EDTA plasma with a Jaffé diagnostic test (Roche Diagnostics). High-density lipoprotein-cholesterol (HDL-cholesterol), total cholesterol and triacylglycerols (TGs) were measured in EDTA plasma as previously described [38]. Low-density lipoprotein cholesterol was estimated with the Friedewald formula [42].

\subsection{Statistical analysis}

Imputation of missing data: Multiple imputation (five imputed data sets) was used to impute missing data in the exposure and confounders in order to decrease bias and to increase the power of the analyses [43]. Missing values in C1q $(n=1), C 1-I N H(n=1), C 4 d(n=1), C 4(n=3)$ and confounders ( $\mathrm{N}=22)$ were imputed. The percentage of missing values per variable varied from $0 \%$ (age, sex, NGM, IGM, T2DM, blood pressure, use of medication, lipids) to $2.1 \%$ (smoking pack years).Missing data in the outcome variables were not imputed (see figure 2). Main analyses: Normally distributed variables are presented as mean \pm standard deviation (SD). Skewed variables (C4d, triacylglycerols, eGFR, biomarkers of inflammation and endothelial dysfunction) are presented as medians with interquartile range (IQR). Skewed variables were $\log _{\mathrm{e}}$-transformed to achieve normal distribution prior to the regression analyses. To provide a robust estimate of inflammation and endothelial dysfunction with minimization of random error, composite scores were calculated of loge-transformed CRP, IL-6, IL-8, TNF $\alpha$, sICAM-1 and SAA for inflammation [44] and of loge-transformed VWF, sEs, sICAM-1 and SVCAM-1 for endothelial dysfunction [45]. For this, each individual biomarker of inflammation or endothelial dysfunction was standardized to the mean of the baseline and follow-up examination (Z-score: [individual value minus mean of all values]/SD of all values). These individual Z-scores were averaged into an inflammation score and an endothelial dysfunction score. To allow for a direct comparison of the effect sizes, baseline C1q, C1-INH and $\log _{e} \mathrm{C} 4 \mathrm{~d}$ were subsequently converted to their respective Z-score. Consequently, in all analyses the associations are presented per 1 SD increase in C1q, C1-INH or logeC4d.

Longitudinal associations of baseline $\mathrm{C} 1 \mathrm{q}, \mathrm{C} 1-\mathrm{INH}$ or $\mathrm{C} 4 \mathrm{~d}$ with inflammation, endothelial dysfunction or IMT were investigated with generalized estimating equations (GEE) using an 
exchangeable correlation structure. Associations of baseline C1q, C1-INH or C4d with incident CVD and CVE in participants free of CVD at baseline were investigated with logistic regression. In all analyses, first non-linearity of any potential relationship was examined by investigating associations of tertiles of $\mathrm{C} 1 \mathrm{q}, \mathrm{C} 1-\mathrm{INH}$ or $\mathrm{C} 4 \mathrm{~d}$ with the respective outcomes. When no evidence for non-linearity was found, $\mathrm{C} 1 \mathrm{q}, \mathrm{C} 1-\mathrm{INH}$ or $\mathrm{C} 4 \mathrm{~d}$ were analysed as continuous variables. If associations of tertiles with an outcome indicated a non-linear relationship, tertiles were used throughout the analyses of that outcome. We also tested if the association of $\mathrm{C} 1 \mathrm{q}, \mathrm{C} 1-\mathrm{INH}$ or $\mathrm{C} 4 \mathrm{~d}$ with inflammation, endothelial dysfunction or IMT changed over time by including an interaction term between time and C1q, C1-INH or C4d in the fully adjusted GEE models. In case of significant interaction with time, GEE analyses were stratified according to baseline and follow-up examination. Such stratified analyses are comparable to cross-sectional and prospective analyses. Furthermore, all analyses were examined for effect modification by sex by including interaction terms between sex and C1q, C1-INH or C4d in the model. If the interaction term was significant, separate analyses among men and women were conducted.

Analyses were first adjusted for variables related to the inclusion procedure, which incorporated stratified sampling for age [years] and sex [men/women], and oversampling of IGM [yes/no] and T2DM [yes/no] (model 1). Subsequently, analyses were adjusted for the following potentially confounding cardiovascular risk factors: waist [cm], systolic blood pressure (SBP) $[\mathrm{mmHg}]$, total cholesterol $[\mathrm{mmol} / \mathrm{L}]$, HDL-cholesterol [mmol/L], triacylglycerols $[\mathrm{mmol} / \mathrm{L}]$, eGFR $\left[\mathrm{ml} \bullet 1.73 \mathrm{~m}^{2} \bullet(\mathrm{min})^{-1}\right]$, smoking [pack years], smoking behaviour [former smoker, current smoker], physical activity [yes/no] and use of antihypertensive [yes/no], lipid-modifying [yes/no], anti-thrombotic [yes/no] or glucoselowering medication [yes/no] (model 2). All adjustments were done with the baseline value of confounders, and all GEE models were additionally adjusted for time of examination [follow-up: yes/no] and follow-up time [years].

Additional analyses: All analyses were repeated with exclusion of participants with a possible acute inflammation (serum CRP concentrations $>10 \mathrm{mg} / \mathrm{L}, \mathrm{n}=38$ at baseline and $\mathrm{n}=42$ at follow-up). Furthermore, complete case analyses were performed. Analyses using only participants with complete data in $\mathrm{C} 1 \mathrm{q}, \mathrm{C} 1-\mathrm{INH}, \mathrm{C} 4 \mathrm{~d}$ and confounders, resulted in $\mathrm{N}=553$ (965 observations) in the GEE analyses of inflammation and endothelial dysfunction, $N=519$ (910 observations) in the GEE analyses of IMT and N=324 in the analyses on incident CVD and CVE. The results were comparable in all analyses, therefore only results obtained with the imputed data sets are presented. In significant associations of C4d with cardiovascular outcomes, we additionally adjusted the fully adjusted model for systemic C4 concentrations in order to evaluate whether associations of $\mathrm{C} 4 \mathrm{~d}$ were independent of its inactive precursor C4.

Assumptions for GEE and logistic regression analyses were met. Statistical analyses were performed using the SPSS package version 22.0 (SPSS, Chicago, IL, USA) and statistical significance was set at $\mathrm{P}<0.05$. 


\section{Results}

\subsection{General characteristics of the study population}

Table 1 shows baseline characteristics of the study population as a whole $(\mathrm{N}=545)$. At baseline, $28 \%$ had CVD and $16 \%$ had CVE. In supplemental table $1 \mathrm{a}-\mathrm{c}$, baseline characteristics across tertiles of $\mathrm{C} 1 \mathrm{q}, \mathrm{C} 1-\mathrm{INH}$ and C4d, respectively, are provided. Overall, C1q, C1-INH and C4d were not consistently associated with an adverse cardiometabolic profile.

The 459 participants with complete follow-up data were at baseline marginally younger ( $59.2 \pm 7.0$ years) compared to the total population at baseline ( $59.6 \pm 7.0$ years) and had a slightly better metabolic profile than the total population (data not shown). Over the 7-year follow-up period, participants increased in inflammation score from $-0.16 \pm 0.90$ to $0.10 \pm 1.05$, increased in endothelial dysfunction score from $-0.12 \pm 0.94$ to $0.08 \pm 1.04$, and increased in cIMT from $768 \pm 157 \mu \mathrm{m}$ to $818 \pm 101 \mu \mathrm{m}$.

Of the 342 individuals who were free of CVD at baseline, 73 developed CVD (21\%), of whom 39 experienced an event (CVE, 11\%) during follow-up. Individuals with incident CVD had at baseline more often T2DM, and were characterized by more inflammation and endothelial dysfunction, and a higher cIMT compared to individuals who remained free of CVD during the follow-up (table 1). 
Table 1: Baseline characteristics of the study population

\begin{tabular}{|c|c|c|c|}
\hline & \multirow{2}{*}{$\begin{array}{c}\text { Total study } \\
\text { population } \\
\mathrm{N}=545^{*}\end{array}$} & \multicolumn{2}{|c|}{ Participants free of CVD at baseline } \\
\hline & & $\begin{array}{c}\text { no CVD at follow-up } \\
\qquad=269\end{array}$ & $\begin{array}{l}\text { CVD at follow-up } \\
\qquad N=73\end{array}$ \\
\hline $\mathrm{C} 1 \mathrm{q}(\mathrm{mg} / \mathrm{L})$ & $72 \pm 15$ & $73 \pm 15$ & $72 \pm 16$ \\
\hline C1-Inhibitor (mg/L) & $170 \pm 12$ & $169 \pm 12$ & $169 \pm 11$ \\
\hline $\mathrm{C} 4 \mathrm{~d}(\mathrm{mg} / \mathrm{L})$ & $1.13[0.70 ; 1.72]$ & $1.11[0.68 ; 1.75]$ & $0.99[0.61 ; 1.64]$ \\
\hline $\mathrm{C} 4(\mathrm{~g} / \mathrm{L})$ & $0.29 \pm 0.07$ & $286 \pm 67$ & $297 \pm 92$ \\
\hline Age (years) & $59.6 \pm 7.0$ & $58 \pm 7$ & $59 \pm 7$ \\
\hline Sex (\%men) & 61 & 60 & 59 \\
\hline NGM/IGM/T2DM (\%) & $52 / 22 / 26$ & $59 / 24 / 17$ & $58 / 16 / 26$ \\
\hline $\mathrm{HbA} 1 \mathrm{c}(\%)$ & $6.0 \pm 0.8$ & $5.84 \pm 0.77$ & $5.90 \pm 0.65$ \\
\hline Waist $(\mathrm{cm})$ & $99 \pm 12$ & $97 \pm 12$ & $99 \pm 13$ \\
\hline Systolic blood pressure (mmHg) & $140 \pm 19$ & $136 \pm 18$ & $140 \pm 18$ \\
\hline Diastolic blood pressure (mmHg) & $82 \pm 9$ & $81 \pm 9$ & $83 \pm 9$ \\
\hline Fasting triacylglycerols (mmol/L) & $1.4[1.0-2.2]$ & $1.40[0.90 ; 1.90]$ & $1.40[0.90 ; 2.00]$ \\
\hline Fasting cholesterol (mmol/L) & $5.2 \pm 1.0$ & $5.28 \pm 0.99$ & $5.29 \pm 0.98$ \\
\hline Fasting HDL cholesterol (mmol/L) & $1.2 \pm 0.3$ & $1.23 \pm 0.35$ & $1.19 \pm 0.36$ \\
\hline Fasting LDL cholesterol (mmol/L) & $3.3 \pm 0.9$ & $3.35 \pm 0.87$ & $3.41 \pm 0.92$ \\
\hline eGFR $\left(\mathrm{ml} / \mathrm{min} / 1.73 \mathrm{~cm}^{2}\right)$ & $94[83-106]$ & $94[84 ; 107]$ & $95[84 ; 104]$ \\
\hline Physically active (\%) & 62 & 63 & 55 \\
\hline Current smokers/former smokers (\%) & $20 / 51$ & $20 / 47$ & $14 / 51$ \\
\hline Pack years of smoking in ex/current smokers & $23[12-36]$ & & \\
\hline Use of anti-hypertensive medication (\%) & 38 & 24 & 38 \\
\hline Use of glucose-lowering medication (\%) & 13 & 7 & 14 \\
\hline Use of lipid-modifying medication (\%) & 19 & 10 & 10 \\
\hline Use of anti-thrombotic medication (\%) & 21 & 5 & 18 \\
\hline $\mathrm{CRP}(\mathrm{mg} / \mathrm{L})$ & $2.07[0.97-3.92]$ & $1.56[0.84 ; 3.21]$ & $2.55[1.02 ; 3.41]$ \\
\hline IL-6 (ng/L) & $1.55[1.13-2.27]$ & $1.34[0.99 ; 1.95]$ & $1.61[1.12 ; 2.22]$ \\
\hline IL-8 (ng/L) & $4.40[3.60-5.56]$ & $4.32[3.47 ; 5.47]$ & $4.19[3.50 ; 5.20]$ \\
\hline TNFa (ng/L) & $6.21[5.26-7.52]$ & $6.08[5.16 ; 7.19]$ & $5.91[5.18 ; 7.30]$ \\
\hline sICAM-1 ( $\mathrm{gg} / \mathrm{L})$ & $0.21[0.19-0.24]$ & 204 [182; 237] & 210 [186; 235] \\
\hline Serum amyloid A (mg/L) & $1.42[0.99-2.26]$ & $1.33[0.94 ; 1.96]$ & $1.49[1.10 ; 2.54]$ \\
\hline Von Willebrand factor (\%) & 119 [91-161] & $113[88 ; 155]$ & $126[91 ; 178]$ \\
\hline Soluble E-selectin ( $\mu \mathrm{g} / \mathrm{L})$ & $8.16[5.98-10.40]$ & $7.85[5.94 ; 9.94]$ & $8.18[5.68 ; 10.10]$ \\
\hline sVCAM-1 ( $\mu \mathrm{g} / \mathrm{L})$ & 332 [293-379] & $324[285 ; 363]$ & 322 [293; 369] \\
\hline Inflammation score & $-0.09 \pm 0.94$ & $-0.34 \pm 0.85$ & $-0.10 \pm 0.92$ \\
\hline Endothelial dysfunction score & $-0.07 \pm 0.96$ & $-0.26 \pm 0.91$ & $-0.09 \pm 0.88$ \\
\hline Carotid IMT ( $\mu \mathrm{m})$ & $774 \pm 157$ & $753 \pm 155$ & $793 \pm 169$ \\
\hline Prevalent CVD (\%) & 28 & - & - \\
\hline Prevalent CVE (\%) & 16 & - & - \\
\hline
\end{tabular}

Data are presented as mean \pm standard deviation (normally distributed variables), median [interquartile range] (skewed variables) or proportion (\%, categorical variables). C1-INH indicates C1-inhibitor; CRP, C-reactive protein; CVD, cardiovascular disease; CVE, cardiovascular events; eGFR, estimated glomerular filtration rate; HbA1c, glycosylated haemoglobin, HDL, high-density lipoprotein; IGM, impaired glucose metabolism; IL-6, interleukin-6; IL-8, interleukin-8; IMT, intima-media thickness; LDL, low-density lipoprotein; NGM, normal glucose metabolism; sICAM-1, soluble intercellular adhesion molecule-1; sVCAM-1, soluble vascular cell adhesion molecule-1; TNF $\alpha$, tumour necrosis factor- $\alpha$; T2DM, type 2 diabetes.

* data before multiple imputation were available in $\mathrm{N}=523(\mathrm{HbA} 1 \mathrm{c})$ to 545 participants. 


\subsection{Associations with incident CVD and CVE}

We observed clear deviations from linearity in associations of C1q with incident CVD and CVE. Therefore C1q was subsequently analysed in tertiles. In contrast, we did not observe deviations from linearity in associations of C1-INH or C4d with the CVD or CVE. Therefore $\mathrm{C} 1-\mathrm{INH}$ and $\mathrm{C} 4 \mathrm{~d}$ were subsequently analysed as continuous variables.

Participants in the second tertile (T2) of C1q had the lowest incidence of CVD; therefore T2 was chosen as reference in the analyses of CVD and CVE (figure 3). In a model adjusted for age, sex, impaired glucose metabolism and T2DM, the OR for CVD of the first tertile (T1) compared to $\mathrm{T} 2$ of $\mathrm{C} 1 \mathrm{q}$ was 2.20 [95\% Cl: 1.10; 4.39], and the OR of the third tertile (T3) compared to T2 was 2.09 [1.05; 4.17]. After adjustment for relevant confounders, the OR of T1 compared to T2 remained significant and the OR for T3 compared to T2 became borderline significant (model 2, T1 compared to T2, OR=2.38 [1.14; 4.95], $P=0.021, \mathrm{~T} 3$ compared to $\mathrm{T} 2, \mathrm{OR}=1.96[0.94 ; 4.07], \mathrm{P}=0.071)$. This suggested a potentially $\mathrm{U}$-shaped relation between $\mathrm{C} 1 \mathrm{q}$ and incident CVD. For incident CVE, a similar pattern was observed. Participants in T2 of $\mathrm{C} 1 \mathrm{q}$ had the lowest incidence of CVE (figure 3), but this was not significantly different from T1 or T3 (model 1, T1 compared to T2, OR=1.66 [0.70; 3.95], $\mathrm{P}=0.253$, T3 compared to $\mathrm{T} 2, \mathrm{OR}=1.55[0.65 ; 3.70], \mathrm{P}=0.326$; model $2, \mathrm{~T} 1$ compared to $\mathrm{T} 2$, $\mathrm{OR}=1.77$ [0.66; 4.79], $\mathrm{P}=0.260$, T3 compared to $\mathrm{T} 2, \mathrm{OR}=1.42$ [0.52; 3.91], $\mathrm{P}=0.499$ ). Associations were comparable in men and women ( $P$-values for interaction $>0.05$ ). Associations were unchanged when participants with a potential acute inflammation (CRP>10 mg/L) were excluded.
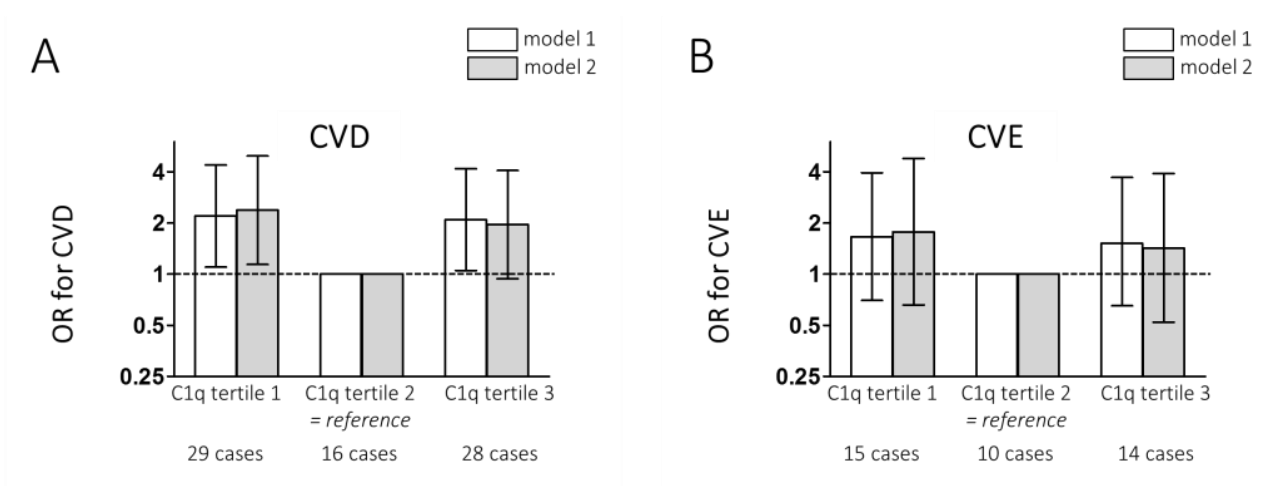

Figure 3: Association of baseline C1q with incident CVD and CVE over the 7-year follow-up. Tertiles of C1q were analysed in 342 participants free of CVD at baseline. Tertile $1(T 1, N=114)$, tertile $2(T 2, N=114)$, tertile $3(N=114)$. Model 1, adjusted for age; sex; impaired glucose metabolism; type 2 diabetes; model 2, additionally adjusted for waist; systolic blood pressure; total cholesterol; HDL-cholesterol; triacylglycerols; eGFR; current smoking; former smoking; smoking pack years; physical activity; use of lipid-modifying medication; use of glucose-lowering medication; use of anti-hypertensive medication; use of anti-thrombotic medication. For detailed effect estimates see text. 
C1-INH was not associated with incident CVD or CVE (table 2, all P-values>0.05). Associations were comparable in men and women (P-values for interaction $>0.05$ ). Associations were unchanged when participants with a potential acute inflammation (CRP>10 mg/L) were excluded.

Table 2: Associations of baseline C1-INH and C4d with incident CVD and CVE

\begin{tabular}{|c|c|c|c|c|}
\hline \multirow[b]{3}{*}{ C1-INH (per 1SD) } & \multicolumn{2}{|c|}{$\begin{array}{c}\text { Incident CVD } \\
\mathrm{N}=342,73 \text { cases }\end{array}$} & \multicolumn{2}{|c|}{$\begin{array}{c}\text { Incident CVE } \\
\mathrm{N}=342,39 \text { cases }\end{array}$} \\
\hline & OR [95\% Cl] & P-value & OR [95\% Cl] & P-value \\
\hline & & & & \\
\hline model 1 & $0.99[0.75 ; 1.31]$ & 0.947 & $0.96[0.66 ; 1.39]$ & 0.820 \\
\hline model 2 & $1.02[0.76 ; 1.38]$ & 0.895 & $1.06[0.69 ; 163]$ & 0.797 \\
\hline \multicolumn{5}{|l|}{$\log _{e}$ C4d (per 1SD) } \\
\hline model 1 & $0.90[0.70 ; 1.16]$ & 0.420 & $0.91[0.65 ; 1.27]$ & 0.258 \\
\hline model 2 & $0.88[0.67 ; 1.16]$ & 0.360 & $0.78[0.53 ; 1.15]$ & 0.209 \\
\hline \multicolumn{5}{|c|}{$\begin{array}{l}\text { OR indicates odds ratio; } 95 \% \mathrm{Cl}, 95 \% \text { confidence interval; CVD, cardiovascular disease; CVE, cardiovascular events; } \\
\text { i.s., interaction term significant. Skewed variables were loge-transformed prior to the analyses. } \\
\text { model 1: age; sex; impaired glucose metabolism; type } 2 \text { diabetes } \\
\text { model 2: model } 1 \text { + waist; systolic blood pressure; total cholesterol; HDL-cholesterol ; triacylglycerols; eGFR; } \\
\text { current smoking; former smoking; smoking pack years; physical activity; use of lipid-modifying medication; use of } \\
\text { glucose-lowering medication; use of anti-hypertensive medication; use of anti-thrombotic medication } \\
\text { * significant interaction terms between loge } C 4 d \text { and sex in the associations with CVD (P-value for } \\
\text { interaction=0.004) and CVE (P-value for interaction=0.003). Associations of loge C4d with CVD and CVE stratified } \\
\text { according to sex are shown in table } 3 .\end{array}$} \\
\hline
\end{tabular}

For C4d, we observed significant effect modification by sex in the associations with CVD (P-value=0.004) and CVE (P-value=0.003), therefore these associations were analysed separately in men and women (table 3). In men, C4d was associated with a lower incidence of CVD and CVE (model 2, OR was 0.59 [0.40; 0.87] for CVD and 0.42 [0.23; 0.75] for CVE). In contrast, in women, C4d appeared to be associated with a higher incidence of CVD and CVE (model 2, CVD: OR=2.14 [1.19; 3.88], CVE: OR=4.68 [1.22; 17.92]). Associations were unchanged when participants with a potential acute inflammation (CRP>10 mg/L) were excluded. 


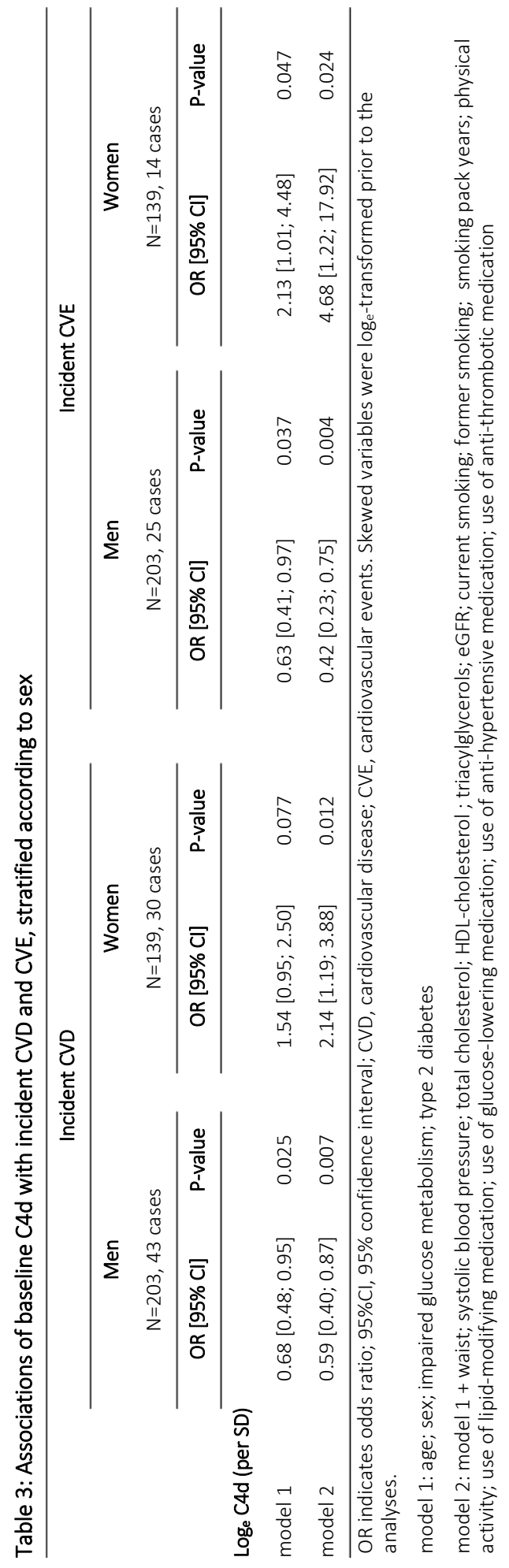




\subsection{Associations with inflammation, endothelial dysfunction and cIMT}

We did not observe deviations from linearity in associations of $\mathrm{C} 1 \mathrm{q}, \mathrm{C} 1-\mathrm{INH}$ or $\mathrm{C} 4 \mathrm{~d}$ with the inflammation score, the endothelial dysfunction score or cIMT. Therefore C1q, C1-INH and c4d were subsequently analysed as continuous variables.

C1q was positively associated with the endothelial dysfunction score over the 7-year followup period (table 4). Since the interaction term between $\mathrm{C} 1 \mathrm{q}$ and time was significant in the associations with the endothelial dysfunction score as well as the inflammation score, baseline and follow-up observations were analysed separately. There was a positive association of $\mathrm{C} 1 \mathrm{q}$ (measured only at baseline) with the baseline inflammation score and the baseline endothelial dysfunction score (table 5, model 2, per 1 SD higher baseline C1q, $\beta$ for the inflammation score was 0.09 [95\% $\mathrm{Cl}: 0.01 ; 0.16], \beta$ for the endothelial dysfunction score was $0.14[0.07 ; 0.21])$. In contrast, there was no association of $\mathrm{C} 1 \mathrm{q}$ with the inflammation or endothelial dysfunction score at follow-up, indicating the lack of a prospective association (all P-values >0.05). C1q was not associated with clMT $(P>0.05)$. Associations were comparable in men and women ( $P$-values for interaction $>0.05$ ) and were unchanged when participants with a potential acute inflammation (CRP>10 $\mathrm{mg} / \mathrm{L}$ ) were excluded.

Table 4: Associations of baseline C1q, C1-INH and C4d with inflammation, endothelial dysfunction and cIMT over the 7-year follow-up period

\begin{tabular}{|c|c|c|c|c|c|c|}
\hline \multirow[b]{3}{*}{ C1q (per 1SL } & \multicolumn{2}{|c|}{$\begin{array}{l}\text { Low-grade inflammation score } \\
\qquad \text { (SD) } \\
\mathrm{N}=572,1045 \text { observations }\end{array}$} & \multicolumn{2}{|c|}{$\begin{array}{l}\text { Endothelial dysfunction score } \\
\text { (SD) } \\
\mathrm{N}=572,1045 \text { observations }\end{array}$} & \multicolumn{2}{|c|}{$\begin{array}{c}\mathrm{clMT} \\
(\mu \mathrm{m}) \\
\mathrm{N}=556,968 \text { observations } \\
\end{array}$} \\
\hline & $\beta[95 \% \mathrm{Cl}]$ & P-value & $\beta[95 \% \mathrm{Cl}]$ & P-value & $\beta[95 \% \mathrm{Cl}]$ & P-value \\
\hline & & & & & & \\
\hline model 1 & $0.09[0.01 ; 0.16]$ & 0.018 & $0.15[0.08 ; 0.22]$ & $<0.001$ & $2[-3 ; 8]$ & 0.669 \\
\hline model 2 & $0.06[-0.01 ; 0.13]$ & 0.109 i.s. $^{*}$ & $0.10[0.03 ; 0.18]$ & 0.006 i.s. $^{*}$ & $1[-9 ; 12]$ & 0.773 \\
\hline \multicolumn{7}{|c|}{ C1-INH (per 1SD) } \\
\hline model 1 & $0.07[-0.00 ; 0.13]$ & 0.066 & $0.02[-0.05 ; 0.10]$ & 0.522 & $7[-2 ; 15]$ & 0.156 \\
\hline model 2 & $0.04[-0.03 ; 0.11]$ & 0.232 & $0.01[-0.06 ; 0.08]$ & 0.727 & $6[-4 ; 15]$ & 0.216 \\
\hline \multicolumn{7}{|c|}{$\log _{e} C 4 d($ per 1SD) } \\
\hline model 1 & $0.12[0.03 ; 0.21]$ & 0.008 & $0.00[-0.10 ; 0.20]$ & 0.505 & $6[-3 ; 16]$ & 0.177 \\
\hline model 2 & $0.05[-0.04 ; 0.13]$ & 0.292 i.s. $^{\#}$ & $-0.08[-0.17 ; 0.02]$ & 0.119 & $0[-9 ; 10]$ & 0.946 \\
\hline
\end{tabular}

$\beta$ indicates the longitudinal regression coefficient over the 7-year follow-up period, as obtained from GEE. $95 \% \mathrm{Cl}, 95 \%$ confidence interval; $\mathrm{C} 1-\mathrm{INH}, \mathrm{C} 1$-inhibitor; $\mathrm{GEE}$, generalized estimating equations; i.s., interaction term significant. Skewed variables were loge-transformed prior to the analyses.

model 1: time of examination; follow-up time; age; sex; impaired glucose metabolism; type 2 diabetes

model 2: model 1 + waist; systolic blood pressure; total cholesterol; HDL-cholesterol ; triacylglycerols; eGFR; current smoking; former smoking; smoking pack years; physical activity; use of lipid-modifying medication; use of glucose-lowering medication; use of anti-hypertensive medication; use of anti-thrombotic medication

* significant interaction terms between $\mathrm{C} 1 \mathrm{q}$ and time in the associations with the low-grade inflammation score ( $P$-value for interaction=0.049) and the endothelial dysfunction score ( $P$-value for interaction=0.024). Stratification of these associations according to time of examination are shown in table 5.

\# significant interaction terms between $\log _{\mathrm{e}} \mathrm{C} 4 \mathrm{~d}$ and sex in the associations with the low-grade inflammation score (P-value for interaction=0.049). Associations of $\log _{e} C 4 d$ with the inflammation score stratified according to sex are given in the text. 
C1-INH tended to be positively associated with the inflammation score in a model adjusted only for age, sex, impaired glucose metabolism and T2DM (table 4, per 1 SD higher C1-INH, $\beta$ was 0.07 [-0.00; 0.13]), but this association was attenuated after adjustment for relevant confounders. C1-INH was not associated with the endothelial dysfunction score or cIMT (all P-values>0.05). There was no interaction between $\mathrm{C} 1-\mathrm{INH}$ and time in the GEE models. Associations were comparable in men and women (P-values for interaction>0.05) and were unchanged when participants with a potential acute inflammation (CRP>10 mg/L) were excluded.

There was no interaction between C4d and time in the GEE models. C4d was not associated with the inflammation score in the whole population (table 4). For C4d, we observed significant effect modification by sex in the associations with the inflammation score ( $P$-value=0.040). Therefore this association was analysed separately in men and women. In men, c4d was in the fully adjusted model positively associated with the inflammation score (per 1SD, $\beta=0.11$ [0.02; $0.20], P=0.018)$, while in women, $C 4 d$ was not associated with the inflammation score (-0.08 [$0.22 ; 0.07], P=0.310)$. When participants with a potential acute inflammation (CRP $>10 \mathrm{mg} / \mathrm{L}$ ) were excluded in the sensitivity analysis, the positive association in men was however attenuated and became borderline significant $(\beta=0.09$ [-0.01; 0.19], $P=0.069)$. C4d was not associated with the endothelial dysfunction score or cIMT (table 4, all P-values>0.05). Associations of $\mathrm{C} 4 \mathrm{~d}$ with the endothelial dysfunction score and with CIMT were comparable in men and women (all P-values for interaction $>0.05$ ).

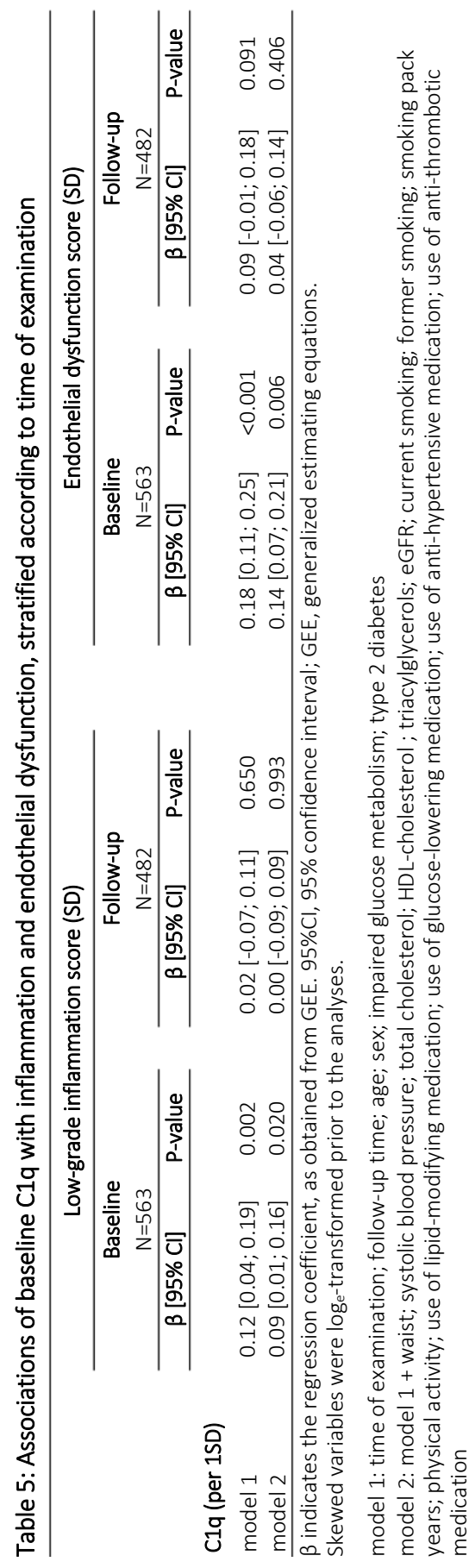




\subsection{Additional analyses}

When we additionally adjusted the associations of C4d with incident CVD and CVE for C4, the inverse associations of C4d with CVD and CVE in men remained significant (CVD, OR=0.56 [0.37; 0.86], $P=0.008 ; C V E, O R=0.35$ [0.17; 0.67], $P=0.002$ ), while the positive associations with CVD and CVE in women were attenuated and became non-significant (CVD, OR=1.60 [0.83; 3.08], $P=0.159 ; C V E, O R=2.42[0.51 ; 11.40], P=0.264)$. When we additionally adjusted the association of $\mathrm{C} 4 \mathrm{~d}$ with the low-grade inflammation score for $\mathrm{C} 4$, the positive association in men became non-significant $(\beta=0.22[-0.17 ; 0.60], P=0.277)$.

\section{Discussion}

This study is the first longitudinal investigation of systemic concentrations of classical pathway factors in human CVD and has several main observations. First, there was a U-shaped association between C1q and CVD. The association between C1q and CVE also appeared to be U-shaped, but was non-significant. Second, we observed significant effect modification by sex in associations of C4d with CVD and CVE. C4d was inversely associated with CVD and CVE in men, while effect estimates were unstable in women. Third, C1q, C1-INH and C4d were not, or inconsistently, associated with LGI, ED and cIMT.

These findings substantially extend previous knowledge. In the present study, we simultaneously measured $\mathrm{C} 1 \mathrm{q}, \mathrm{C} 1-\mathrm{INH}$ and $\mathrm{C} 4 \mathrm{~d}$. This allows a more complete evaluation of determinants of classical pathway activation in human CVD compared with previous studies that each measured a single factor. In our prospective study we observed a U-shaped association of C1q with future CVD. This may at first sight appear to contrast with previous studies that did not report an association between C1q and prevalent CVD [17, 18]. However, those previous studies were cross-sectional, considerably smaller than our cohort, and did not explore a non-linear relationship. Our sex-stratified analyses suggested that classical pathway activation, as represented by $\mathrm{C} 4 \mathrm{~d}$, may have a different effect on CVD in men and women. Notably, previous studies did not address these associations in men and women separately [19]. The current study is also the first to investigate whether the classical pathway is associated with low-grade inflammation and/or endothelial dysfunction. In this respect, the absence of positive associations between $\mathrm{C4d}$ and inflammation or endothelial dysfunction is noteworthy given that complement activation is generally thought to promote inflammation and vascular dysfunction.

The risk to develop CVD was lowest in the second tertile of baseline C1q concentrations. Apparently, both low and high systemic concentrations of $\mathrm{C} 1 \mathrm{q}$ are related to adverse processes in CVD. Such a dual role may be explained by protective as well as pathological effects of C1q in CVD, as was also suggested by experimental studies [2-4]. We did not observe similar U-shaped associations between $\mathrm{C} 1 \mathrm{q}$ and inflammation, endothelial dysfunction or CIMT. Therefore, the observed U-shaped association with CVD is unlikely to be explained by U-shaped associations with these processes underlying the aetiology of CVD. Notably, several in vitro studies have suggested additional mechanisms that might underlie 
a dual relationship with CVD. On the one hand, C1q was reported to direct macrophage polarization towards a more anti-inflammatory $\mathrm{M} 2$-like phenotype [46-48]. Accordingly, upon vascular injury C1q may promote a regulatory, anti-inflammatory immune response, which might have a beneficial effect in CVD. In the current study, we could only investigate the association of $\mathrm{C} 1 \mathrm{q}$ with proinflammatory cytokines, but, unfortunately, not with antiinflammatory or regulatory cytokines such as interleukin-10. On the other hand, C1q may reflect adverse metabolic effects. In mice, C1q was implicated in induction of adipose tissue inflammation and insulin resistance [16]. In agreement, we observed in our population that C1q correlated with a higher prevalence of T2DM, with higher triacylglycerols and lower HDLcholesterol (supplemental data, table 1). In our view, beneficial effects of $\mathrm{C} 1 \mathrm{q}$ in immune regulation combined with simultaneous adverse effects on metabolism and general lowgrade inflammation could underlie the U-shaped association of C1q with CVD observed in the current study.

We did not observe an association between C1q and inflammation or endothelial dysfunction over time. Baseline C1q was positively associated with baseline markers of inflammation and endothelial dysfunction, which suggests that plasma $\mathrm{C} 1 \mathrm{q}$ concentrations reflect inflammation and endothelial dysfunction only in the short-term. In contrast, baseline C1q was not associated with markers of inflammation and endothelial dysfunction seven years later. One possible explanation is that C1q may be causally involved in inflammation and endothelial dysfunction, but that imprecision in the measurements may have introduced bias towards null, and may have especially weakened the prospective association. Alternatively, lack of a prospective association could be interpreted as argument against causality in this relation. Such a reverse causation scenario is conceivable. $\mathrm{C} 1 \mathrm{q}$ is produced extrahepatically by macrophages, adipocytes and endothelial cells $[6,49,50]$ and secretion of C1q by macrophages was observed to be increased by proinflammatory stimuli [4]. As such, higher systemic C1q concentrations may, at least partly, be the result of ongoing inflammatory processes or endothelial activation and not vice versa.

We did not observe an association of C1q with atherosclerosis as reflected by cIMT. This was in line with the only previous but substantially smaller human study on C1q and cIMT [27]. Notably, cIMT reflects only thickening of the arterial wall and our findings do not preclude a role of $\mathrm{C} 1 \mathrm{q}$ in other aspects of atherosclerosis, such as in adverse arterial remodelling, plaque burden or plaque phenotype [51,52]. Both $\mathrm{C} 1 \mathrm{q}$ and $\mathrm{C} 1 \mathrm{q}$-receptors were found in human atherosclerotic plaques [53, 54], which supports relevance of $\mathrm{C} 1 \mathrm{q}$ also in human atherosclerosis. Animal studies suggested a protective effect of $\mathrm{C} 1 \mathrm{q}$ in atherosclerosis. In mice, genetic deficiency of $\mathrm{C} 1 \mathrm{q}$ resulted in more atherosclerosis due to impaired clearance of apoptotic cells. Moreover, C1q may also be produced locally by infiltrated immune cells [53], which suggests that plasma concentrations might insufficiently reflect local, potentially more relevant effects of $\mathrm{C} 1 \mathrm{q}$ in the vascular wall. This may explain the discrepancy between human and animal data with regard to atherosclerosis, and also the discrepancy between observations on cIMT and CVD/CVE in the current study.

C1-INH was not independently associated with any cardiovascular outcome. C1-INH itself is known to have potent anti-inflammatory effects that are already used therapeutically to 
treat acute inflammatory attacks in hereditary angioedema [28, 55]. We had therefore anticipated inverse associations of $\mathrm{C} 1-\mathrm{INH}$ with adverse cardiovascular outcomes, especially with inflammation. The lack of an association of C1-INH with inflammation in our study thus suggests that the anti-inflammatory properties of C1-INH are of minor relevance in obesityassociated chronic low-grade inflammation. The only previous human studies on C1-INH and CVD included patients with angina pectoris and had inconsistent findings [20,21]. Notably, besides acting as the main inhibitor of complement activation downstream of $\mathrm{C} 1 \mathrm{q}, \mathrm{C} 1-\mathrm{INH}$ also inhibits proteases of the lectin pathway and of the contact system of coagulation [25]. Furthermore, C1-INH may have additional biological activities independent of its function as protease inhibitor [56]. But taken together, systemic concentrations of C1-INH do in our study apparently not reflect relevant functions of C1-INH in development of CVD.

Associations of C4d with CVD and CVE were substantially different between men and women. This observation might potentially contribute to a better understanding of the known sex differences in risk factors and presentation of CVD $[57,58]$. At this point in time, we can, however, not conclusively explain how higher $\mathrm{C} 4 \mathrm{~d}$ could be protective in development of CVD in men, but not in women. In our view, the positive associations of C4d with CVD and CVE observed in women should be interpreted with caution. In women, the number of cases in the analyses of CVD and CVE was relatively small, and we observed wide confidence intervals around the effect estimates for CVD and CVE. Furthermore, these positive associations in women were not independent of $\mathrm{C} 4$, which is the inactive precursor of $\mathrm{C} 4 \mathrm{~d}$; while in men, inverse associations with CVD and CVE were independent of C4. When we considered female sex hormones, i.e. menopausal status, hormone replacement therapy or use of oral contraceptives as confounders or effect modifiers in our analyses, our findings were unaltered (data not shown). In previous studies on C4d and CVD or T2DM, results were not reported separately for men and women $[19,59]$. These studies were relatively small and may therefore not have specifically addressed possible differences between men and women. Also, to the best of our knowledge, most experimental studies on the contribution of the classical pathway to CVD included only male mice $[12,13,60]$. Noteworthy, we measured C4d as marker of classical pathway activation, but C4d is also released during activation of the lectin pathway. It is therefore possible, that associations of C4d partly represent events of lectin pathway activation. Notwithstanding, our findings of a protective effect in men and a null effect in women indicate that higher complement activation at the level of C4, irrespective whether arising from classical or lectin pathway activation, is not harmful in the development of CVD.

Our study is the hitherto most comprehensive study on the classical pathway in human CVD and has several strengths but also limitations. The greatest strength is its prospective design, which allowed us to investigate associations with incident CVD and CVE. Importantly, our study was large enough to investigate potential non-linear associations and to investigate associations in men and women separately. Furthermore, the detailed phenotyping of the participants allowed us to investigate different processes underlying the development of CVD. The most relevant limitation of our study is its observational nature. Furthermore, laboratory measures of $\mathrm{C} 1 \mathrm{q}, \mathrm{C} 1-\mathrm{INH}$ and $\mathrm{C} 4 \mathrm{~d}$ are not standardized and consequently, 
reported concentrations vary between studies. Yet, our study is a single-centre cohort with a standardized sample processing and storage protocol. Furthermore, we acknowledge the relatively high variation in the $\mathrm{C} 1 \mathrm{q}$ measurement, despite the use of a well-described, published assay [25]. This may have introduced random error in the C1q concentrations. Importantly, this can only have resulted in underestimation of the reported associations [61]. Also, the power in the analysis of incident CVD and especially incident CVE was relatively low. This may explain why the associations between C1q and CVD were significant, while the associations between $\mathrm{C} 1 \mathrm{q}$ and CVE showed a similar but non-significant pattern. Finally, our study cohort was selected for a moderately increased risk of cardiometabolic disease, which might limit the generalizability of our results. Notwithstanding, our cohort represents a large part of typical Western populations, namely middle-aged to elderly individuals that are overweight, modestly hypertensive, have some signs of a disturbed glucose metabolism and/or use medication for various reasons.

In summary, both high and low plasma concentrations of the pattern recognition molecule C1q were associated with an approximately 2 to 2.5 -fold higher risk to develop CVD, with a similar trend for CVE. This suggests a U-shaped relationship of C1q with risk of CVD and implies that C1q may have both protective and pathological effects in CVD. Furthermore, we observed, in men, inverse associations of C4d with CVD and CVE. This suggests that higher activation of the classical pathway may be protective in disease mechanisms that are particularly relevant in CVD in men. In conclusion, elevated systemic levels of C1q, C1-INH and C4d do overall not reflect an adverse cardiovascular profile. Rather, our findings support the concept that the classical pathway may have both protective and pathological effects in development of CVD. 


\section{References}

1. Hertle E, Stehouwer CD, van Greevenbroek MM. The complement system in human cardiometabolic disease. Mol. Immunol., 2014. 61: 135-48.

2. Speidl WS, Kastl SP, Huber K, Wojta J. Complement in atherosclerosis: friend or foe? J. Thromb. Haemost., 2011. 9: 428-40.

3. Haskard DO, Boyle JJ, Mason JC. The role of complement in atherosclerosis. Curr. Opin. Lipidol., 2008. 19: 47882.

4. Bohlson SS, Fraser DA, Tenner AJ. Complement proteins C1q and MBL are pattern recognition molecules that signal immediate and long-term protective immune functions. Mol. Immunol., 2007. 44: 33-43.

5. Gal P, Dobo J, Zavodszky P, Sim RB. Early complement proteases: C1r, C1s and MASPs. A structural insight into activation and functions. Mol. Immunol., 2009. 46: 2745-52.

6. Trouw LA, Blom AM, Gasque P. Role of complement and complement regulators in the removal of apoptotic cells. Mol. Immunol., 2008. 45: 1199-207.

7. Degn SE, Thiel S. Humoral pattern recognition and the complement system. Scand. J. Immunol., 2013. 78: 18193.

8. Nayak A, Pednekar L, Reid KB, Kishore U. Complement and non-complement activating functions of C1q: a prototypical innate immune molecule. Innate immunity, 2012. 18: 350-63.

9. Fraser DA, Bohlson SS, Jasinskiene N, Rawal N, Palmarini G, Ruiz S, Rochford R, Tenner AJ. C1q and MBL, components of the innate immune system, influence monocyte cytokine expression. J. Leukoc. Biol., 2006. 80: 107-16.

10. Beinrohr L, Dobo J, Zavodszky P, Gal P. C1, MBL-MASPs and C1-inhibitor: novel approaches for targeting complement-mediated inflammation. Trends Mol. Med., 2008. 14: 511-21.

11. Ghebrehiwet B, Jesty J, Vinayagasundaram R, Vinayagasundaram U, Ji Y, Valentino A, Tumma N, Hosszu KH, Peerschke El. Targeting gC1qR domains for therapy against infection and inflammation. Adv. Exp. Med. Biol., 2013. 735: 97-110.

12. Bhatia VK, Yun S, Leung V, Grimsditch DC, Benson GM, Botto MB, Boyle JJ, Haskard DO. Complement C1q reduces early atherosclerosis in low-density lipoprotein receptor-deficient mice. Am. J. Pathol., 2007. 170: 416-26.

13. Lewis MJ, Malik TH, Ehrenstein MR, Boyle JJ, Botto M, Haskard DO. Immunoglobulin M is required for protection against atherosclerosis in low-density lipoprotein receptor-deficient mice. Circulation, 2009. 120: 417-26.

14. Shagdarsuren E, Bidzhekov K, Djalali-Talab Y, Liehn EA, Hristov M, Matthijsen RA, Buurman WA, Zernecke A, Weber C. C1-esterase inhibitor protects against neointima formation after arterial injury in atherosclerosisprone mice. Circulation, 2008. 117: 70-8.

15. Yin W, Ghebrehiwet B, Weksler B, Peerschke El. Classical pathway complement activation on human endothelial cells. Mol. Immunol., 2007. 44: 2228-34.

16. Hillian AD, McMullen MR, Sebastian BM, Roychowdhury S, Kashyap SR, Schauer PR, Kirwan JP, Feldstein AE, Nagy LE. Mice lacking C1q are protected from high fat diet-induced hepatic insulin resistance and impaired glucose homeostasis. J. Biol. Chem., 2013. 288: 22565-75.

17. Hirata A, Kishida K, Nakatsuji H, Kobayashi H, Funahashi T, Shimomura I. High serum C1q-adiponectin/total adiponectin ratio correlates with coronary artery disease in Japanese type 2 diabetics. Metabolism, 2013. 62: 578-85.

18. Kishida K, Nakagawa Y, Kobayashi H, Yanagi K, Funahashi T, Shimomura I. Increased serum C1q-binding adiponectin complex to total-adiponectin ratio in men with multi-vessel coronary disease. Diabetol. Metab. Syndr., 2014. 6: 64 [doi: 10.1186/1758-5996-6-64].

19. Yasuda M, Takeuchi K, Hiruma M, lida H, Tahara A, Itagane H, Toda I, Akioka K, Teragaki M, Oku H. The complement system in ischemic heart disease. Circulation, 1990. 81: 156-163.

20. Danese C, Marciano F, Ciarla MV, Colotto M, Angrisani L, Ferranti E, Borgia MC. [Evaluation of C3c, C4 and C1esterase inhibitor (C1-INH) during unstable angina]. Minerva Cardioangiol., 2002. 50: 667-72.

21. Kostner KM, Fahti RB, Case C, Hobson P, Tate J, Marwick TH. Inflammation, complement activation and endothelial function in stable and unstable coronary artery disease. Clin. Chim. Acta, 2006. 365: 129-34.

22. Davis AE, 3rd, Mejia P, Lu F. Biological activities of C1 inhibitor. Mol. Immunol., 2008. 45: 4057-63.

23. Kruijshoop M, Feskens EJ, Blaak EE, de Bruin TW. Validation of capillary glucose measurements to detect glucose intolerance or type 2 diabetes mellitus in the general population. Clin. Chim. Acta, 2004. 341: 33-40.

24. Hertle E, van Greevenbroek MM, Arts IC, van der Kallen CJ, Geijselaers SL, Feskens EJ, Jansen EH, Schalkwijk CG, Stehouwer CD. Distinct associations of complement C3a and its precursor C3 with atherosclerosis and cardiovascular disease. The CODAM study. Thromb. Haemost., 2014. 111: 1102-11. 
25. Dillon SP, D'Souza A, Kurien BT, Scofield RH. Systemic lupus erythematosus and C1q: A quantitative ELISA for determining C1q levels in serum. Biotechnol. J., 2009. 4: 1210-4.

26. Bergseth G, Ludviksen JK, Kirschfink M, Giclas PC, Nilsson B, Mollnes TE. An international serum standard for application in assays to detect human complement activation products. Mol. Immunol., 2013. 56: 232-9.

27. Hirata A, Kishida K, Kobayashi H, Nakatsuji H, Funahashi T, Shimomura I. Correlation between serum C1qadiponectin/total adiponectin ratio and polyvascular lesions detected by vascular ultrasonography in Japanese type 2 diabetics. Metabolism, 2013. 62: 376-85.

28. Wouters D, Wagenaar-Bos I, van Ham M, Zeerleder S. C1 inhibitor: just a serine protease inhibitor? New and old considerations on therapeutic applications of C1 inhibitor. Expert Opin. Biol. Ther., 2008. 8: 1225-40.

29. Fuentes-Arderiu X, Alonso-Gregorio E, Alvarez-Funes V, Ambros-Marigomez C, Coca-Fabregas L, Cruz-Placer M, Diaz-Fernandez J, Pinel-Julian MP, Gutierrez-Cecchini B, Herrero-Bernal P, Sempere-Alcocer M, GarciaCaballero F, Del Mar Larrea-Ortiz-Quintana M, La-Torre-Marcellan P, Del Senor Lopez-Velez M, Mar-Medina C, Martin-Oncina J, Rodriguez-Hernandez MV, Romero-Sotomayor MV, Serrano-Lopez C, Sicilia-Enriquez-deSalamanca A, Velasco-Romero AM, Juve-Cuxart S. Multicentre physiological reference intervals for serum concentrations of immunoglobulins A, G and M, complement C3c and C4 measured with Tina-Quant reagents systems. Clin. Chem. Lab. Med., 2007. 45: 387-90.

30. Jacobs M, van Greevenbroek MM, van der Kallen CJ, Ferreira I, Blaak EE, Feskens EJ, Jansen EH, Schalkwijk CG, Stehouwer CD. Low-grade inflammation can partly explain the association between the metabolic syndrome and either coronary artery disease or severity of peripheral arterial disease: the CODAM study. Eur. J. Clin. Invest., 2009. 39: 437-44.

31. Jacobs M, van Greevenbroek MM, van der Kallen CJ, Ferreira I, Blaak EE, Feskens EJ, Jansen EH, Schalkwijk CG, Stehouwer $\mathrm{CD}$. The association between the metabolic syndrome and peripheral, but not coronary, artery disease is partly mediated by endothelial dysfunction: the CODAM study. Eur. J. Clin. Invest., 2011. 41: 16775.

32. van Bussel BC, Ferreira I, van de Waarenburg MP, van Greevenbroek MM, van der Kallen CJ, Henry RM, Feskens EJ, Stehouwer CD, Schalkwijk CG. Multiple Inflammatory Biomarker Detection in a Prospective Cohort Study: A Cross-Validation between Well-Established Single-Biomarker Techniques and an Electrochemiluminescense-Based Multi-Array Platform. PLoS ONE, 2013. 8: e58576.

33. Stam F, van Guldener C, Schalkwijk CG, ter Wee PM, Donker AJ, Stehouwer CD. Impaired renal function is associated with markers of endothelial dysfunction and increased inflammatory activity. Nephrol. Dial. Transplant., 2003. 18: 892-898.

34. Engelen L, Ferreira I, Gaens KH, Henry RM, Dekker JM, Nijpels G, Heine RJ, t Hart LM, van Greevenbroek MM, van der Kallen CJ, Blaak EE, Feskens EJ, Ten Cate H, Stehouwer CD, Schalkwijk CG. The association between the -374T/A polymorphism of the receptor for advanced glycation endproducts gene and blood pressure and arterial stiffness is modified by glucose metabolism status: the Hoorn and CoDAM studies. J. Hypertens., 2010. 28: 285-93.

35. Aboyans V, Criqui MH, Abraham P, Allison MA, Creager MA, Diehm C, Fowkes FG, Hiatt WR, Jonsson B, Lacroix P, Marin B, McDermott MM, Norgren L, Pande RL, Preux PM, Stoffers HE, Treat-Jacobson D. Measurement and interpretation of the ankle-brachial index: a scientific statement from the American Heart Association. Circulation, 2012. 126: 2890-909.

36. Atsma F, Bartelink ML, Grobbee DE, van der Schouw YT. Best reproducibility of the ankle-arm index was calculated using Doppler and dividing highest ankle pressure by highest arm pressure. J. Clin. Epidemiol., 2005. 58: 1282-8.

37. Alberti KG, Zimmet PZ. Definition, diagnosis and classification of diabetes mellitus and its complications. Part 1: diagnosis and classification of diabetes mellitus provisional report of a WHO consultation. Diabet. Med., 1998. 15: 539-53.

38. van Greevenbroek MM, Jacobs M, van der Kallen CJ, Blaak EE, Jansen EH, Schalkwijk CG, Feskens EJ, Stehouwer $\mathrm{CD}$. Human plasma complement C3 is independently associated with coronary heart disease, but only in heavy smokers (the CODAM study). Int. J. Cardiol., 2012. 154: 158-62.

39. Wendel-Vos GC, Schuit AJ, Saris WH, Kromhout D. Reproducibility and relative validity of the short questionnaire to assess health-enhancing physical activity. J. Clin. Epidemiol., 2003. 56: 1163-9.

40. Kemper HC, Ooijendijk WT, Stiggelbout M. Consensus over de Nederlandse Norm voor Gezond Bewegen. Tijdschr. Soc. Gezondheidsz., 2000. 78: 180-183.

41. Levey AS, Bosch JP, Lewis JB, Greene T, Rogers N, Roth D. A more accurate method to estimate glomerular filtration rate from serum creatinine: a new prediction equation. Modification of Diet in Renal Disease Study Group. Ann. Intern. Med., 1999. 130: 461-70.

42. Friedewald WT, Levy RI, Fredrickson DS. Estimation of the concentration of low-density lipoprotein cholesterol in plasma, without use of the preparative ultracentrifuge. Clin. Chem., 1972. 18: 499-502.

43. Sterne JA, White IR, Carlin JB, Spratt M, Royston P, Kenward MG, Wood AM, Carpenter JR. Multiple imputation for missing data in epidemiological and clinical research: potential and pitfalls. Brit. Med. J., 2009. 338: b2393. 
44. van Bussel BC, Schouten F, Henry RM, Schalkwijk CG, de Boer MR, Ferreira I, Smulders YM, Twisk JW, Stehouwer CD. Endothelial dysfunction and low-grade inflammation are associated with greater arterial stiffness over a 6-year period. Hypertension, 2011. 58: 588-95.

45. Hertle E, van Greevenbroek MM, Arts IC, van der Kallen CJ, Feskens EJ, Schalkwijk CG, Stehouwer CD. Complement activation products $\mathrm{C} 5 \mathrm{a}$ and $\mathrm{s} \mathrm{C} 5 \mathrm{~b}-9$ are associated with low-grade inflammation and endothelial dysfunction, but not with atherosclerosis in a cross-sectional analysis: The CODAM study. Int. J. Cardiol., 2014. 174: 400-3.

46. Bohlson SS, O'Conner SD, Hulsebus HJ, Ho MM, Fraser DA. Complement, c1q, and c1q-related molecules regulate macrophage polarization. Front. Immunol., 2014. 5: 402.

47. Spivia W, Magno PS, Le P, Fraser DA. Complement protein C1q promotes macrophage anti-inflammatory M2like polarization during the clearance of atherogenic lipoproteins. Inflamm. Res., 2014. 63: 885-93.

48. Clarke EV, Weist BM, Walsh CM, Tenner AJ. Complement protein C1q bound to apoptotic cells suppresses human macrophage and dendritic cell-mediated Th17 and Th1 T cell subset proliferation. J. Leukoc. Biol., 2015. 97: 147-60.

49. Morgan BP, Gasque P. Extrahepatic complement biosynthesis: where, when and why? Clin. Exp. Immunol., 1997. 107: 1-7.

50. Ghebrehiwet B, Hosszu KK, Valentino A, Peerschke EI. The C1q family of proteins: insights into the emerging non-traditional functions. Front. Immunol., 2012. 3.

51. Ward MR, Pasterkamp G, Yeung AC, Borst C. Arterial remodeling. Mechanisms and clinical implications. Circulation, 2000. 102: 1186-91.

52. Heusch G, Libby P, Gersh B, Yellon D, Bohm M, Lopaschuk G, Opie L. Cardiovascular remodelling in coronary artery disease and heart failure. Lancet, 2014. 383: 1933-43.

53. Cao W, Bobryshev YV, Lord RS, Oakley RE, Lee SH, Lu J. Dendritic cells in the arterial wall express C1q: potential significance in atherogenesis. Cardiovasc. Res., 2003. 60: 175-86.

54. Peerschke EI, Minta JO, Zhou SZ, Bini A, Gotlieb A, Colman RW, Ghebrehiwet B. Expression of gC1q-R/p33 and its major ligands in human atherosclerotic lesions. Mol. Immunol., 2004. 41: 759-66.

55. Sharp JA, Whitley PH, Cunnion KM, Krishna NK. Peptide inhibitor of complement c1, a novel suppressor of classical pathway activation: mechanistic studies and clinical potential. Front. Immunol., 2014. 5: 406.

56. Davis AE, 3rd, Cai S, Liu D. C1 inhibitor: biologic activities that are independent of protease inhibition. Immunobiology, 2007. 212: 313-23.

57. Maas AH, Appelman YE. Gender differences in coronary heart disease. Neth. Heart J., 2010. 18: 598-602.

58. Yahagi K, Davis HR, Arbustini E, Virmani R. Sex differences in coronary artery disease: pathological observations. Atherosclerosis, 2015. 239: 260-7.

59. Fujita T, Hemmi S, Kajiwara M, Yabuki M, Fuke Y, Satomura A, Soma M. Complement-mediated chronic inflammation is associated with diabetic microvascular complication. Diabetes Metab. Res. Rev., 2013. 29: 220-6.

60. Francescut L, Steiner T, Byrne S, Cianflone K, Francis S, Stover C. The role of complement in the development and manifestation of murine atherogenic inflammation: novel avenues. J. Innate Immun., 2012. 4: 260-72.

61. Hutcheon JA, Chiolero A, Hanley JA. Random measurement error and regression dilution bias. Brit. Med. J., 2010. 340: c2289. 


\section{Supplemental data}

\section{Supplemental table 1: Characteristics across tertiles of C1q, C1-INH or C4d}

\begin{tabular}{|c|c|c|c|}
\hline \multicolumn{4}{|l|}{ 1a) Characteristics according to tertiles of $\mathrm{C} 1 \mathrm{q}$} \\
\hline & $\begin{array}{l}\text { Lowest tertile } \\
\qquad \mathrm{n}=189 \\
31-64 \mathrm{mg} / \mathrm{L}\end{array}$ & $\begin{array}{c}\text { Middle tertile } \\
\quad n=190 \\
64-78 \mathrm{mg} / \mathrm{L}\end{array}$ & $\begin{array}{l}\text { Highest tertile } \\
\mathrm{n}=190 \\
78-129 \mathrm{mg} / \mathrm{L}\end{array}$ \\
\hline $\mathrm{C} 1 \mathrm{q}(\mathrm{mg} / \mathrm{L})$ & $56 \pm 6$ & $71 \pm 4$ & $90 \pm 10$ \\
\hline C1-Inhibitor (mg/L) & $171 \pm 13$ & $169 \pm 12$ & $170 \pm 12$ \\
\hline $\mathrm{C} 4 \mathrm{~d}(\mathrm{mg} / \mathrm{L})$ & $1.18[0.68 ; 1.68]$ & $1.11[0.74 ; 1.62]$ & $1.12[0.72 ; 1.87]$ \\
\hline $\mathrm{C} 4$ (g/L) & $0.29 \pm 0.07$ & $0.28 \pm 0.07$ & $0.30 \pm 0.08$ \\
\hline Age (years) & $60 \pm 7$ & $59 \pm 7$ & $60 \pm 7$ \\
\hline Sex (\%men) & 65 & 59 & 60 \\
\hline NGM/IGM/T2DM (\%) & $60 / 21 / 19$ & $49 / 21 / 30$ & $48 / 25 / 27$ \\
\hline HbA1c (\%) & $5.98 \pm 0.76$ & $5.99 \pm 0.80$ & $5.96 \pm 0.90$ \\
\hline Waist $(\mathrm{cm})$ & $99 \pm 12$ & $100 \pm 12$ & $100 \pm 12$ \\
\hline Systolic blood pressure (mmHg) & $139 \pm 19$ & $140 \pm 19$ & $142 \pm 20$ \\
\hline Diastolic blood pressure ( $\mathrm{mmHg}$ ) & $81 \pm 8$ & $82 \pm 9$ & $83 \pm 11$ \\
\hline Fasting triacylglycerols (mmol/L) & $1.30[0.90 ; 1.90]$ & $1.40[1.00 ; 1.93]$ & $1.50[1.08 ; 2.00]$ \\
\hline Fasting cholesterol (mmol/L) & $5.20 \pm 1.04$ & $5.22 \pm 0.82$ & $5.21 \pm 1.04$ \\
\hline Fasting HDL cholesterol (mmol/L) & $1.27 \pm 0.34$ & $1.18 \pm 0.36$ & $1.13 \pm 0.32$ \\
\hline Fasting LDL cholesterol (mmol/L) & $3.25 \pm 0.91$ & $3.36 \pm 0.77$ & $3.30 \pm 0.90$ \\
\hline eGFR $\left(\mathrm{ml} / \mathrm{min} / 1.73 \mathrm{~cm}^{2}\right)$ & $93[83 ; 106]$ & $97[87 ; 108]$ & $92[80 ; 104]$ \\
\hline Physically active (\%) & 61 & 62 & 64 \\
\hline Current smokers/former smokers (\%) & $23 / 52$ & $20 / 48$ & $18 / 52$ \\
\hline Pack years of smoking in ex/current smokers & $23[11 ; 43]$ & $23[14 ; 34]$ & $23[11 ; 38]$ \\
\hline Use of anti-hypertensive medication (\%) & 34 & 36 & 45 \\
\hline Use of glucose-lowering medication (\%) & 12 & 13 & 16 \\
\hline Use of lipid-modifying medication (\%) & 19 & 16 & 22 \\
\hline Use of anti-thrombotic medication (\%) & 20 & 18 & 25 \\
\hline $\mathrm{CRP}(\mathrm{mg} / \mathrm{L})$ & $1.54[82 ; 3.19]$ & $2.31[0.99 ; 4.49]$ & $2.29[1.14 ; 4.42]$ \\
\hline IL-6 (ng/L) & $1.51[1.03 ; 2.06]$ & $1.54[1.20 ; 2.38]$ & $1.68[1.16 ; 2.40]$ \\
\hline IL-8 (ng/L) & $4.39[3.44 ; 5.67]$ & $4.34[3.74 ; 5.58]$ & $4.42[3.70 ; 5.56]$ \\
\hline TNF $\alpha$ (ng/L) & $5.96[5.16 ; 7.40]$ & $6.19[5.16 ; 7.66]$ & $6.38[5.51 ; 7.62]$ \\
\hline sICAM-1 $(\mu \mathrm{g} / \mathrm{L})$ & $204[184 ; 237]$ & 215 [191; 249] & $219[192 ; 251]$ \\
\hline Serum amyloid A (mg/L) & $1.39[0.95 ; 1.86]$ & $1.44[1.03 ; 2.41]$ & $1.51[0.94 ; 2.59]$ \\
\hline Von Willebrand factor (\%) & $117[88 ; 157]$ & $119[92 ; 157]$ & $125[88 ; 168]$ \\
\hline Soluble E-selectin ( $\mu \mathrm{g} / \mathrm{L})$ & $8.03[5.77 ; 10.11]$ & $8.06[6.42 ; 10.49]$ & $8.29[6.44 ; 10.58]$ \\
\hline sVCAM-1 ( $\mu \mathrm{g} / \mathrm{L})$ & $319[286 ; 361]$ & $328[284 ; 365]$ & 351 [309; 405] \\
\hline Inflammation score & $-0.25 \pm 0.90$ & $-0.08 \pm 0.97$ & $0.05 \pm 0.96$ \\
\hline Endothelial dysfunction score & $-0.25 \pm 0.87$ & $-0.09 \pm 0.93$ & $0.15 \pm 1.03$ \\
\hline Carotid IMT ( $\mu \mathrm{m})$ & $783 \pm 161$ & $762 \pm 144$ & $777 \pm 166$ \\
\hline Prevalent CVD (\%) & 27 & 28 & 28 \\
\hline Prevalent CVE (\%) & 16 & 13 & 18 \\
\hline
\end{tabular}

Data are presented as mean \pm standard deviation (normally distributed variables), median [interquartile range] (skewed variables) or proportion (\%, categorical variables). C1-INH indicates C1-inhibitor; CRP, C-reactive protein; CVD, cardiovascular disease; CVE, cardiovascular events; eGFR, estimated glomerular filtration rate; HbA1c, glycosylated haemoglobin, HDL, high-density lipoprotein; IGM, impaired glucose metabolism; IL-6, interleukin-6; IL-8, interleukin-8; IMT, intima-media thickness; LDL, low-density lipoprotein; NGM, normal glucose metabolism; sICAM-1, soluble intercellular adhesion molecule-1; sVCAM-1, soluble vascular cell adhesion molecule-1; TNF $\alpha$, tumour necrosis factor- $\alpha$; T2DM, type 2 diabetes.

* data before multiple imputation were available in $\mathrm{N}=523$ ( $\mathrm{HbA1c}$ ) to 545 participants. 


\section{1b) Characteristics according to tertiles of C1-INH}

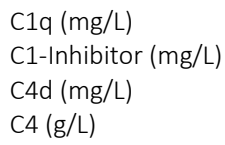

Age (years)

Sex (\%men)

NGM/IGM/T2DM (\%)

HbA1c (\%)

Waist $(\mathrm{cm})$

Systolic blood pressure $(\mathrm{mmHg})$

Diastolic blood pressure $(\mathrm{mmHg})$

Fasting triacylglycerols ( $\mathrm{mmol} / \mathrm{L})$

Fasting cholesterol (mmol/L)

Fasting HDL cholesterol ( $\mathrm{mmol} / \mathrm{L})$

Fasting LDL cholesterol ( $\mathrm{mmol} / \mathrm{L})$

eGFR $\left(\mathrm{ml} / \mathrm{min} / 1.73 \mathrm{~cm}^{2}\right)$

Physically active (\%)

Current smokers/former smokers (\%)

Pack years of smoking in ex/current smokers

Use of anti-hypertensive medication (\%)

Use of glucose-lowering medication (\%)

Use of lipid-modifying medication (\%)

Use of anti-thrombotic medication (\%)

$$
\begin{aligned}
& \text { CRP (mg/L) } \\
& \text { IL-6 (ng/L) } \\
& \text { IL-8 (ng/L) } \\
& \text { TNF } \alpha \text { (ng/L) } \\
& \text { sICAM-1 ( } \mathrm{mg} / \mathrm{L})
\end{aligned}
$$

Serum amyloid $A(\mathrm{mg} / \mathrm{L})$

Von Willebrand factor (\%)

Soluble E-selectin ( $\mu \mathrm{g} / \mathrm{L})$

sVCAM-1 ( $\mu \mathrm{g} / \mathrm{L})$

Inflammation score

\begin{tabular}{|c|c|c|}
\hline $\begin{array}{l}\text { Lowest tertile } \\
\quad n=189\end{array}$ & $\begin{array}{l}\text { Middle tertile } \\
n=190\end{array}$ & $\begin{array}{c}\text { Highest tertile } \\
n=190\end{array}$ \\
\hline $109-164 \mathrm{mg} / \mathrm{L}$ & $164-174 \mathrm{mg} / \mathrm{L}$ & $175-205 \mathrm{mg} / \mathrm{L}$ \\
\hline $73 \pm 16$ & $72 \pm 14$ & $72 \pm 17$ \\
\hline $157 \pm 7$ & $170 \pm 3$ & $183 \pm 7$ \\
\hline $0.97[0.67 ; 1.60]$ & $1.08[0.71 ; 1.66]$ & $1.35[0.82 ; 1.99]$ \\
\hline $0.28 \pm 0.07$ & $0.29 \pm 0.07$ & $0.31 \pm 0.08$ \\
\hline $58 \pm 7$ & $60 \pm 7$ & $61 \pm 7$ \\
\hline 69 & 62 & 53 \\
\hline $55 / 23 / 22$ & $55 / 19 / 26$ & $47 / 25 / 28$ \\
\hline $5.85 \pm 0.81$ & $6.01 \pm 0.83$ & $6.08 \pm 0.81$ \\
\hline $100 \pm 12$ & $99 \pm 12$ & $100 \pm 12$ \\
\hline $136 \pm 16$ & $141 \pm 20$ & $143 \pm 21$ \\
\hline $81 \pm 9$ & $82 \pm 9$ & $82 \pm 9$ \\
\hline $1.40[1.00 ; 1.90]$ & $1.40[1.00 ; 2.00]$ & $1.40[1.00 ; 2.00]$ \\
\hline $5.13 \pm 0.96$ & $5.24 \pm 0.85$ & $5.26 \pm 1.08$ \\
\hline $1.13 \pm 0.34$ & $1.24 \pm 0.37$ & $1.20 \pm 0.32$ \\
\hline $3.32 \pm 0.88$ & $3.28 \pm 0.82$ & $3.30 \pm 0.88$ \\
\hline $94[86 ; 104]$ & $93[83 ; 109]$ & $94[83 ; 106]$ \\
\hline 64 & 61 & 64 \\
\hline $18 / 51$ & $19 / 57$ & $24 / 44$ \\
\hline $20[10 ; 36]$ & $23[21 ; 34]$ & $26[13 ; 42]$ \\
\hline 33 & 39 & 43 \\
\hline 12 & 11 & 17 \\
\hline 15 & 19 & 23 \\
\hline 24 & 21 & 18 \\
\hline
\end{tabular}

Endothelial dysfunction score

Carotid IMT $(\mu \mathrm{m})$

Prevalent CVD (\%)

Prevalent CVE (\%)

$$
\begin{gathered}
1.64[0.83 ; 3.22] \\
1.52[1.11 ; 2.21] \\
4.31[3.49 ; 5.66] \\
6.08[5.19 ; 7.40] \\
205[185 ; 244] \\
1.25[0.88 ; 1.95] \\
117[87 ; 152] \\
8.03[5.77 ; 10.14] \\
325[286 ; 371] \\
-0.25 \pm 0.94 \\
-0.18 \pm 1.05 \\
755 \pm 147 \\
23 \\
14
\end{gathered}
$$

$2.21[1.01 ; 3.80]$

$1.52[1.10 ; 2.23]$

$4.46[3.70 ; 5.62]$

$6.17[5.29 ; 7.54]$

212 [187; 244]

1.35 [0.99; 2.27]

$122[93 ; 170]$

$8.06[6.16 ; 10.65]$

329 [290; 377]

$-0.07 \pm 0.92$

$-0.02 \pm 0.96$

$784 \pm 167$

26

14
$2.32[1.13 ; 4.69]$

$1.62[1.22 ; 2.38]$

$4.40[3.60 ; 5.39]$

$6.31[5.27 ; 7.62]$

218 [192; 246]

$1.68[1.10 ; 2.71]$

$121[90 ; 158]$

8.22 [6.00; 10.39]

346 [299; 394]

$0.05 \pm 0.96$

$0.01 \pm 0.86$

$783 \pm 155$

33

18

Data are presented as mean \pm standard deviation (normally distributed variables), median [interquartile range] (skewed variables) or proportion (\%, categorical variables). C1-INH indicates C1-inhibitor; CRP, C-reactive protein; CVD, cardiovascular disease; CVE, cardiovascular events; eGFR, estimated glomerular filtration rate; HbA1c, glycosylated haemoglobin, HDL, high-density lipoprotein; IGM, impaired glucose metabolism; IL-6, interleukin-6; IL-8, interleukin-8; IMT, intima-media thickness; LDL, low-density lipoprotein; NGM, normal glucose metabolism; sICAM-1, soluble intercellular adhesion molecule-1; sVCAM-1, soluble vascular cell adhesion molecule-1; TNF $\alpha$, tumour necrosis factor- $\alpha$; T2DM, type 2 diabetes.

* data before multiple imputation were available in $\mathrm{N}=523$ ( $\mathrm{HbA} 1 \mathrm{c})$ to 545 participants. 


\section{1c) Characteristics according to tertiles of C4d}

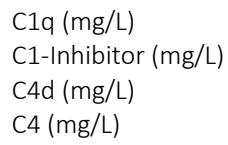

Age (years)

Sex (\%men)

NGM/IGM/T2DM (\%)

HbA1c (\%)

Waist $(\mathrm{cm})$

Systolic blood pressure $(\mathrm{mmHg})$

Diastolic blood pressure $(\mathrm{mmHg})$

Fasting triacylglycerols ( $\mathrm{mmol} / \mathrm{L})$

Fasting cholesterol (mmol/L)

Fasting HDL cholesterol ( $\mathrm{mmol} / \mathrm{L})$

Fasting LDL cholesterol ( $\mathrm{mmol} / \mathrm{L})$

eGFR $\left(\mathrm{ml} / \mathrm{min} / 1.73 \mathrm{~cm}^{2}\right)$

Physically active (\%)

Current smokers/former smokers (\%)

Pack years of smoking in ex/current smokers

Use of anti-hypertensive medication (\%)

Use of glucose-lowering medication (\%)

Use of lipid-modifying medication (\%)

Use of anti-thrombotic medication (\%)

$$
\begin{aligned}
& \text { CRP (mg/L) } \\
& \mathrm{IL}-6 \text { (ng/L) } \\
& \mathrm{IL}-8 \text { (ng/L) } \\
& \text { TNF } \alpha \text { (ng/L) } \\
& \text { sICAM-1 ( } \mathrm{mg} / \mathrm{L})
\end{aligned}
$$

Serum amyloid $A(\mathrm{mg} / \mathrm{L})$

Von Willebrand factor (\%)

Soluble E-selectin ( $\mu \mathrm{g} / \mathrm{L})$

sVCAM-1 ( $\mu \mathrm{g} / \mathrm{L})$

Endothelial dysfunction score

Carotid IMT $(\mu \mathrm{m})$

Prevalent CVE (\%)
Inflammation score

Prevalent CVD (\%)

\begin{tabular}{ccc} 
Lowest tertile & Middle tertile & Highest tertile \\
$\mathrm{n}=190$ & $\mathrm{n}=190$ & $\mathrm{n}=189$ \\
$0.16-0.82 \mathrm{mg} / \mathrm{L}$ & $0.83-1.52 \mathrm{mg} / \mathrm{L}$ & $1.53-13.09 \mathrm{mg} / \mathrm{L}$ \\
\hline $72 \pm 16$ & $71 \pm 15$ & $73 \pm 15$ \\
$168 \pm 12$ & $171 \pm 11$ & $170 \pm 13$ \\
$0.59[0.48 ; 0.71]$ & $1.13[0.97 ; 1.34]$ & $2.12[1.72 ; 2.75]$ \\
$259 \pm 57$ & $590 \pm 72$ & $324 \pm 71$ \\
& & \\
$59 \pm 7$ & $61 \pm 7$ & $59 \pm 7$ \\
63 & 63 & 59 \\
$45 / 24 / 31$ & $57 / 20 / 23$ & $54 / 24 / 22$ \\
$6.00 \pm 0.87$ & $5.99 \pm 0.86$ & $5.95 \pm 0.73$ \\
$99 \pm 11$ & $99 \pm 11$ & $99 \pm 12$ \\
$140 \pm 19$ & $140 \pm 19$ & $141 \pm 20$ \\
$81 \pm 9$ & $82 \pm 9$ & $83 \pm 9$ \\
$1.40[0.90 ; 1.90]$ & $1.45[1.00 ; 2.03]$ & $1.40[1.00 ; 1.90]$ \\
$5.12 \pm 0.94$ & $5.31 \pm 0.98$ & $5.21 \pm 0.98$ \\
$1.20 \pm 0.38$ & $1.18 \pm 0.33$ & $1.20 \pm 0.32$ \\
$3.22 \pm 0.83$ & $3.36 \pm 0.85$ & $3.32 \pm 0.91$ \\
$97[85 ; 108]$ & $92[82 ; 104]$ & $93[81 ; 106]$ \\
60 & 70 & 60 \\
$21 / 52$ & $18 / 56$ & $22 / 44$ \\
$23[11 ; 39]$ & $24[12 ; 34]$ & $20[12 ; 38]$ \\
38 & 37 & 40 \\
17 & 10 & 13 \\
15 & 18 & 24 \\
23 & 20 & 21
\end{tabular}

$\begin{array}{ccc}1.89[0.99 ; 4.02] & 2.07[0.91 ; 3.94] & 2.15[0.97 ; 3.91] \\ 1.59[1.18 ; 2.30] & 1.52[1.12 ; 2.24] & 1.55[1.11 ; 2.27] \\ 4.40[3.70 ; 5.88] & 4.53[3.68 ; 5.62] & 4.21[3.47 ; 5.36] \\ 6.29[5.35 ; 7.63] & 6.11[5.23 ; 7.49] & 6.23[5.02 ; 7.55] \\ 217[191 ; 243] & 213[189 ; 250] & 208[184 ; 235] \\ 1.29[0.98 ; 2.09] & 1.48[1.01 ; 2.27] & 1.49[0.96 ; 2.43] \\ 118[86 ; 156] & 121[92 ; 164] & 121[92 ; 166] \\ 8.78[6.04 ; 10.62] & 7.87[6.07 ; 10.19] & 8.06[5.89 ; 10.38] \\ 334[294 ; 379] & 331[290 ; 382] & 3278[290 ; 381] \\ -0.03 \pm 0.93 & -0.10 \pm 0.97 & -0.14 \pm 0.94 \\ -0.01 \pm 0.99 & -0.05 \pm 0.94 & -0.12 \pm 0.95 \\ 765 \pm 154 & 777 \pm 164 & 780 \pm 155 \\ 25 & 30 & 28 \\ 15 & 15 & 16\end{array}$

Data are presented as mean \pm standard deviation (normally distributed variables), median [interquartile range] (skewed variables) or proportion (\%, categorical variables). C1-INH indicates C1-inhibitor; CRP, C-reactive protein; CVD, cardiovascular disease; CVE, cardiovascular events; eGFR, estimated glomerular filtration rate; HbA1c, glycosylated haemoglobin, HDL, high-density lipoprotein; IGM, impaired glucose metabolism; IL-6, interleukin-6; IL-8, interleukin-8; IMT, intima-media thickness; LDL, low-density lipoprotein; NGM, normal glucose metabolism; sICAM-1, soluble intercellular adhesion molecule-1; sVCAM-1, soluble vascular cell adhesion molecule-1; TNF $\alpha$, tumour necrosis factor- $\alpha$; T2DM, type 2 diabetes.

${ }^{*}$ data before multiple imputation were available in $\mathrm{N}=523$ (HbA1c) to 545 participants. 


\section{Chapter 8 CusbfGr 8}

Longitudinal associations of MBL, MASP-1, MASP-2, MASP-3 and MAp44 with cardiovascular outcomes: The CODAM study

submitted for publication

E. Hertle, I.C.W. Arts, C.J.H. van der Kallen, E.J.M. Feskens, C.G. Schalkwijk, S. Thiel,

I. Hoffmann-Petersen, C.D.A. Stehouwer, M.M.J. van Greevenbroek 


\begin{abstract}
Objective

Previous studies have suggested a complex role of the lectin complement pathway in cardiovascular disease (CVD). There are no prospective human studies on the relationship of the initiating factor of the lectin pathway, i.e. mannose-binding lectin (MBL) with low-grade inflammation (LGI), endothelial dysfunction (ED) or carotid intima-media thickness (cIMT). Moreover, MBL-associated proteases (MASPs) and MBL-associated proteins (MAps), which mediate downstream complement activation, have not been studied in development of CVD. Approach and results

In a prospective cohort ( $N=574$, age $60 \pm 7$ years, 7 years follow-up), we investigated longitudinal associations of plasma MBL, MASP-1, MASP-2, MASP-3 and MAp44 with biomarker scores reflecting LGI and ED, and with CIMT; and investigated their associations with incident CVD. In adjusted analyses, LGI was lowest in the middle tertile ( $T_{\text {Middle) }}$ of MBL. TLow was 0.19 SD [0.03; 0.34] higher than TMiddle, and THigh was 0.15 SD [-0.02; 0.31] higher than TMiddle, suggesting a U-shaped relationship. cIMT was $28 \mu \mathrm{m}[-50 ;-5]$ lower in $T_{\text {High }}$ than in $T_{\text {Middle, }}$ while cIMT did not differ between $\mathrm{T}_{\text {Low }}$ and $\mathrm{T}_{\text {Middle. }} \mathrm{MBL}$ was not associated with ED or CVD. MASP-1 and MASP-2 were not associated with LGI, ED, CIMT or CVD. MASP-3 and MAp44 were independently of MBL associated with ED (per 1 SD, MASP-3: $\beta=0.10$ SD [0.02; 0.18]; MAp44: $\beta=0.12$ SD [0.04; 0.20]), but not with LGI, cIMT or CVD.
\end{abstract}

\title{
Conclusions
}

MBL may have a dual role in low-grade inflammation and contribute to low cIMT. MASP-1 and MASP-2 were not associated with adverse cardiovascular outcomes. MASP-3 and MAp44 may play a role in endothelial dysfunction, potentially independently of lectin pathway activation. 


\section{Introduction}

The immune system, and more specifically to the present report, the lectin pathway of complement activation, have been suggested to have a complex role in cardiovascular disease (CVD) [1]. The lectin pathway is initiated when foreign structures are recognized by soluble pattern-recognition molecules, one of which is mannose-binding lectin (MBL) [2]. Several large studies have investigated the role of MBL in CVD, but have yielded contradictory findings. Studies that investigated MBL genotypes as proxy for circulating MBL consistently found that genotypes resulting in low or deficient $M B L$ were a risk factor for the development of CVD [3-6]. This harmful effect of diminished MBL was explained by the role of MBL in the clearance of pathogens and apoptotic cells in the vessel wall [7-9]. However, when MBL concentrations instead of genotypes were investigated, most studies observed positive [1012] rather than inverse [13] associations with future CVD. Besides, MBL was suggested to be a key pathological mechanism in complications of established CVD. Wildtype genotypes and high MBL concentrations were associated with worse outcomes in most studies [14-16]. The discrepancy between these observations suggests a dual role of $\mathrm{MBL}$ in the pathophysiology of CVD.

To gain insight into the complex role of the lectin pathway in CVD, a more comprehensive evaluation of the lectin pathway is needed. Firstly, a better understanding of the role of $\mathrm{MBL}$ in different etiological aspects of CVD is required. Only few human studies have investigated $\mathrm{MBL}$ in low-grade inflammation, endothelial dysfunction and atherosclerosis. A previous study on carotid intima-media thickness (cIMT) observed a U-shaped association of MBL concentrations with cIMT [17]. However, this study was rather small $(\mathrm{N}=114)$ and included only patients with rheumatoid arthritis. Secondly, factors that mediate downstream complement activation through $\mathrm{MBL}$ should be considered as well. MBL circulates in plasma as a complex with MBL-associated proteases (MASPs) and MBL-associated proteins (MAps) [8]. MASPs are proteases with different substrates involved in activation of both the lectin and the alternative complement pathway $[18,19]$. MAps are thought to have regulatory functions in complement activation $[18,19]$. Currently, little is known about the function of MASPs and MAps in CVD. In humans, MASPs and MAps have so far only been measured in case-control studies or during acute cardiovascular events [20-22], but there are no longitudinal studies on MASPs and MAps in development of CVD.

For these reasons, we determined MBL concentrations as well as genotypes, and concentrations of MASP-1, MASP-2, MASP-3 and MAp44 in a prospective human cohort. We investigated their longitudinal associations with biomarker scores reflecting inflammation and endothelial dysfunction, and with cIMT as a marker of atherosclerosis. Furthermore, we determined their associations with incident CVD and cardiovascular events (CVE) over a 7-year follow-up period. Previous studies suggested both beneficial as well as adverse effects of $\mathrm{MBL}$ in CVD, which may yield non-linear associations with cardiovascular outcomes. Therefore, we checked for deviations from linearity in all analyses. 


\section{Methods}

\subsection{Study design and participants}

The Cohort on Diabetes and Atherosclerosis Maastricht (CODAM) is a prospective cohort study [23]. Briefly, participants were selected from a population-based cohort study if they were Caucasian, aged $>40$ years, and met at least one of the following criteria: $B M \mid>25$ $\mathrm{kg} / \mathrm{m} 2$, use of anti-hypertensive medication, a positive family history of type 2 diabetes, history of gestational diabetes and/or glycosuria [24]. 574 participants were included and extensively phenotyped. After a median follow-up of 7.0 years [interquartile range (IQR) 6.97.1 years], 495 individuals participated in follow-up measurements (for details see flowchart, figure 1). The study was approved by the Medical Ethics Committee of the Maastricht University Medical Centre. All participants gave written informed consent.

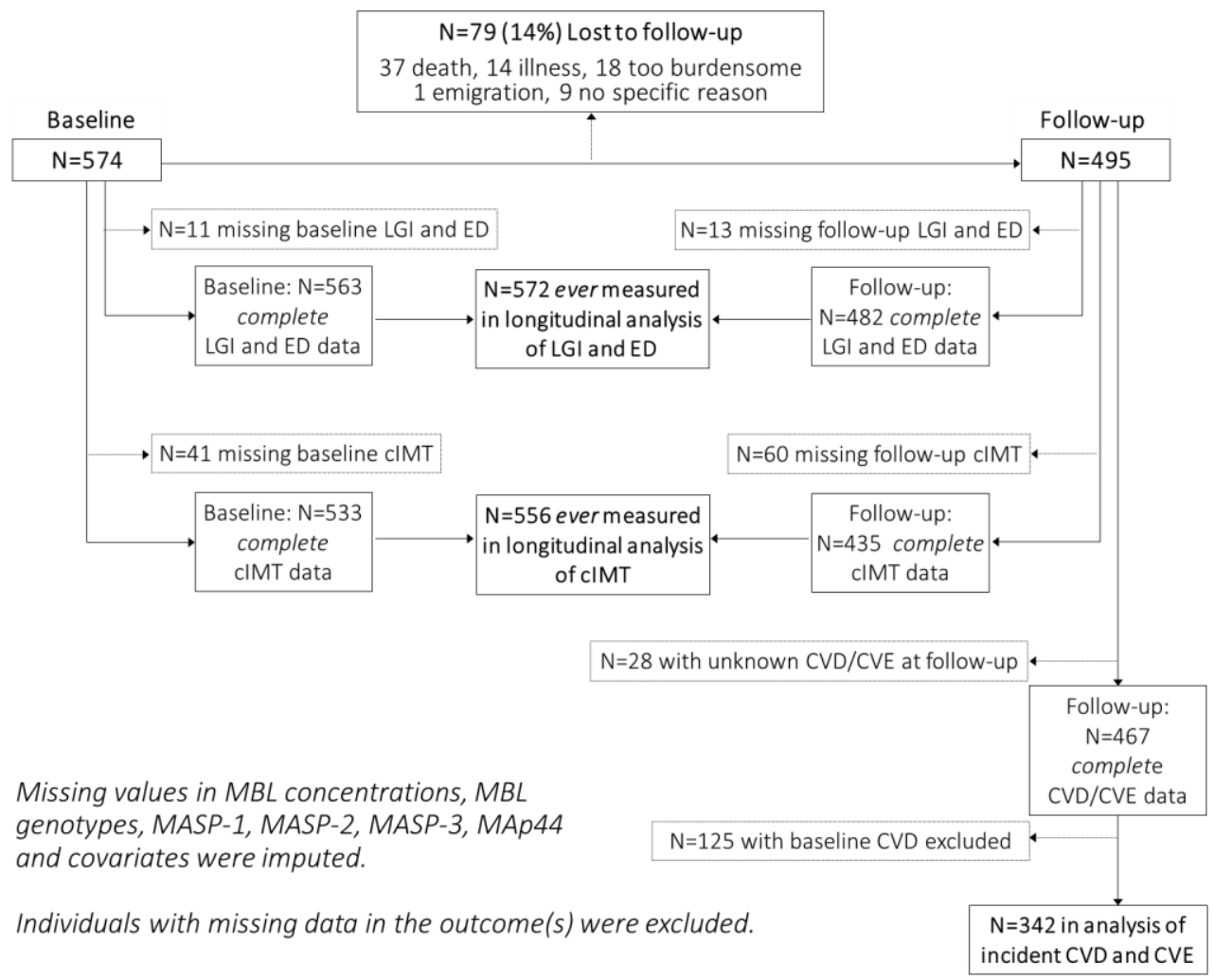

Figure 1: Flow-chart of study participants. CVD, cardiovascular disease; CVE, cardiovascular events; LGI, low-grade inflammation; ED, endothelial dysfunction; cIMT, intima-media thickness. $\mathrm{N}=572$ ever measured in longitudinal analysis of $\mathrm{LGI}$ and $\mathrm{ED}$, of which $\mathrm{N}=473$ have complete data at both baseline and follow-up, $\mathrm{N}=90$ have complete data only at baseline and $\mathrm{N}=9$ have complete data only at follow-up, adding up to 1045 observations included in the GEE analysis of LGI and ED. $\mathrm{N}=556$ ever measured in longitudinal analysis of $\mathrm{cIMT}$, of which $\mathrm{N}=412$ have complete data at both baseline and follow-up, $\mathrm{N}=121$ have complete data only at baseline and $\mathrm{N}=23$ have complete data only at followup, adding up to 968 observations included in the GEE analysis of cIMT. For the analyses with incident CVE and CVD, persons with prevalent CVD at baseline were excluded. 


\subsection{Measurements}

Participants were asked to stop lipid-modifying medication 14 days prior to blood withdrawal, while other medication was stopped one day beforehand. Venous blood samples were collected by venipuncture after an overnight fast. Plasma aliquots were stored at $-80^{\circ} \mathrm{C}$ until use.

Complement factors (measured at baseline): MBL was measured in EDTA plasma by ELISA (MBL ELISA kit, Hycult Biotech, catalogue number HK323, Uden, The Netherlands). MASP-1, MASP-2, MASP-3 and MAp44 were measured in citrate plasma with ELISA (MASP-2 with MASP-2 ELISA kit, Hycult Biotech, catalogue number HK326; MASP-1, MASP-3 and MAp44 with in-house time-resolved immunofluorometric assays as described in [25]). Inter-assay coefficients of variation were $12 \%$ for MBL, $8 \%$ for MASP-1, $13 \%$ for MASP-2, $13 \%$ for MASP-3 and $6 \%$ for MAp44. Median plasma MBL concentration was in our study lower than in two large population-based studies $[10,13]$, but was similar compared to two other cohorts of middle-aged to older individuals with T2DM $[5,26]$. Concentrations of MASP-1, MASP-3 and MAp44 were similar as reported in previous studies, while concentrations of MASP-2 were of the same order of magnitude [20-22]. C3a, C5a and sC5b-9 were measured as described previously [24, 27].

MBL genotypes: DNA was isolated from peripheral blood buffy coats (obtained from EDTAanticoagulated blood) by QIAamp DNA Mini Kit (Quiagen, Germantown, MD, USA). Single nucleotide polymorphisms in the $\mathrm{mb} / 2$ gene at codon 52 (D-allele, rs5030737) and codon 57 (C-allele, rs1800451) were detected by polymerase chain reaction (PCR) using the primers 5'CCAGGGATGGGTCATCTATTT 3' (forward) and 5'TGCCAGAGAATGAGAGCTGA 3' (reverse). After amplification, the PCR products were digested with the restriction endonucleases Banl and Mboll, and subsequently separated on a $2 \%$ agarose gel and visualized by ethidiumbromide staining. Furthermore, all CODAM participants have been genotyped using the Illumina HumanOmniExpress BeadChip assay (Illumina, San Diego, CA, USA). MBL polymorphisms at codon 54 (B-allele, rs1800450) as well as at codon 52 (D-allele, rs5030737) was extracted from the curated dataset. For the polymorphism at codon 52 (D-allele, rs5030737) the identity in the genotypes detected by PCR or as extracted from the HumanOmniExpress dataset was $>99 \%$. The presence of exclusively wildtype alleles was designated as $A / A$ genotype, the presence of one variant allele $(B, C$, or $D)$ was designated as $A / 0$ genotype, and the homozygous presence of one variant allele as well as the heterozygous presence of two variant alleles (compound heterozygosity) was designated as 0/0 [28, 29]. The distribution of genotypes was comparable to previous studies, such as a large population-based study of Caucasians or a study of patients with T2DM [4, 30].

Biomarkers of inflammation and endothelial dysfunction (measured at baseline and followup): C-reactive protein (CRP), interleukin-6, soluble intercellular adhesion molecule-1 (sICAM-1), serum amyloid A, soluble E-selectin and soluble vascular cell adhesion molecule1 (sVCAM-1) were measured twice in the baseline samples: once with individual ELISA assays in serum or EDTA plasma [31, 32], and once paired with the follow-up samples plasma using a multiarray detection system (MesoScale Discovery, SECTOR Imager 2400, Gaithersburg, Maryland, USA). The two baseline measurements of CRP, interleukin-6, sICAM-1, serum 
amyloid A, soluble E-selectin and SVCAM-1 were calibrated after cross-validation as described [33], and the mean of two measurements was used for analyses. Paired measurements of baseline and follow-up interleukin-8 and tumour necrosis factor- $\alpha$ were done in EDTA plasma using a multiarray detection system (MesoScale Discovery, SECTOR Imager 2400) [33]. Baseline and follow-up von Willebrand factor was measured with an inhouse ELISA in citrated plasma as described [34], and the concentration was expressed as percentage of normal pooled plasma.

CIMT, incident CVD and CVE: CIMT was measured by a trained vascular sonographer at both the left and right common carotid artery 10-20 mm proximal to the carotid bulb with a noninvasive ultrasound imaging device (at baseline with Ultramark 4+, Advance Technology Laboratories, Bothel, Washington, USA [35]; at follow-up with Picus, ESAOTE, Maastricht, Netherlands). CVD was defined as the occurrence of at least one of the following: previous myocardial infarction; coronary bypass; percutaneous coronary intervention $(\mathrm{PCI})$ reported by questionnaires (subjects' disease history questionnaire); signs on a 12-lead electrocardiogram of myocardial infarction (Minnesota codes 1-1 or 1-2) or ischaemia (Minnesota codes 1-3, 4-1, 4-2, 4-3, 5-1, 5-2, 5-3 or 7-1); stroke or transient ischaemic attack reported by questionnaire; non-traumatic limb amputation; and/or an ankle-brachial index $<0.9$ [36]. The ankle-brachial index was determined as previously described [32, 37]. This CVD definition was established to obtain a reference population that was truly free of CVD. This may, however, have resulted in identification of a heterogeneous group of cases, ranging from subclinical disease (e.g. stable atherosclerosis) to clinical events (atherothrombosis). Therefore we also investigated cardiovascular events (CVE), which comprised myocardial infarction, stroke, coronary bypass and/or PCl.

Other covariates: normal glucose metabolism, impaired glucose metabolism (impaired glucose metabolism, combining impaired fasting glucose, and impaired glucose tolerance) and T2DM were identified with a standard $75 \mathrm{~g}$ oral glucose tolerance test according to the WHO criteria of 1999 [38]. Waist and blood pressure were measured as reported previously [39]. Smoking, habitual physical activity, use of anti-hypertensive, glucose-lowering, antithrombotic and lipid-modifying medication was assessed with on-site administered questionnaires $[39,40]$. Use of anti-thrombotic medication was defined as use of either antiplatelet agents or coumarin derivatives. Participants were defined as physically active if they were compliant with the Dutch physical activity guideline [41]. Estimated glomerular filtration rate (eGFR) was calculated with the MDRD formula [42] using creatinine concentrations, measured in EDTA plasma with a Jaffé diagnostic test (Roche Diagnostics). High-density lipoprotein-cholesterol (HDL-cholesterol), total cholesterol and triacylglycerols were measured in EDTA plasma as previously described [39]. Low-density lipoproteincholesterol (LDL-cholesterol) was estimated with the Friedewald formula [43]. Homeostasis model assessment of insulin resistance was determined as previously described [44]. 


\subsection{Statistical analysis}

Multiple imputation (five imputed data sets) was used to impute missing data in the exposure and potential confounders in order to decrease bias and to increase the power of the analyses [45]. Missing values in MBL $(n=7)$, MBL-genotypes ( $N=8)$, MASP-1 $(N=11)$, MASP-2 $(\mathrm{N}=9), \operatorname{MASP}-3(\mathrm{~N}=11)$ and MAp44 $(\mathrm{N}=7)$ and confounders $(\mathrm{N}=22)$ were imputed. The percentage of missing values per variable varied from $0 \%$ (age, sex, impaired glucose metabolism, T2DM, blood pressure, use of medication, lipids) to $2.1 \%$ (smoking pack years). Missing data in the outcome variables were not imputed (see figure 2).

Normally distributed variables are presented as mean \pm standard deviation (SD). Skewed variables (triacylglycerols, eGFR, biomarkers of inflammation and endothelial dysfunction) and plasma $\mathrm{MBL}$ are presented as medians with interquartile range (IQR). Skewed variables were $\log _{\mathrm{e}}$-transformed to achieve normal distribution prior to the regression analyses. $\mathrm{MBL}$ was not normally distributed (figure 2). A part of the population had no detectable $\mathrm{MBL}$ protein, and in participants with detectable $M B L$, the distribution of $M B L$ was skewed to the right. Due to MBL-deficient participants, normal distribution of MBL cannot be achieved by standard loge-transformation. Therefore, plasma MBL was in all regression associations analysed as tertiles. To provide a robust estimate of inflammation and endothelial dysfunction with minimization of random error, composite scores were calculated of $\log _{\mathrm{e}^{-}}$ transformed CRP, interleukin-6, interleukin-8, tumour necrosis factor- $\alpha$, sICAM-1 and serum

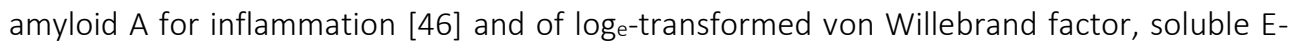
selectin, sICAM-1 and SVCAM-1 for endothelial dysfunction [27]. For this, each individual biomarker of inflammation or endothelial dysfunction was standardized to the mean of the baseline and follow-up examination (Z-score: [individual value minus mean of all values]/SD of all values). These individual Z-scores were averaged into an inflammation score and an endothelial dysfunction score.

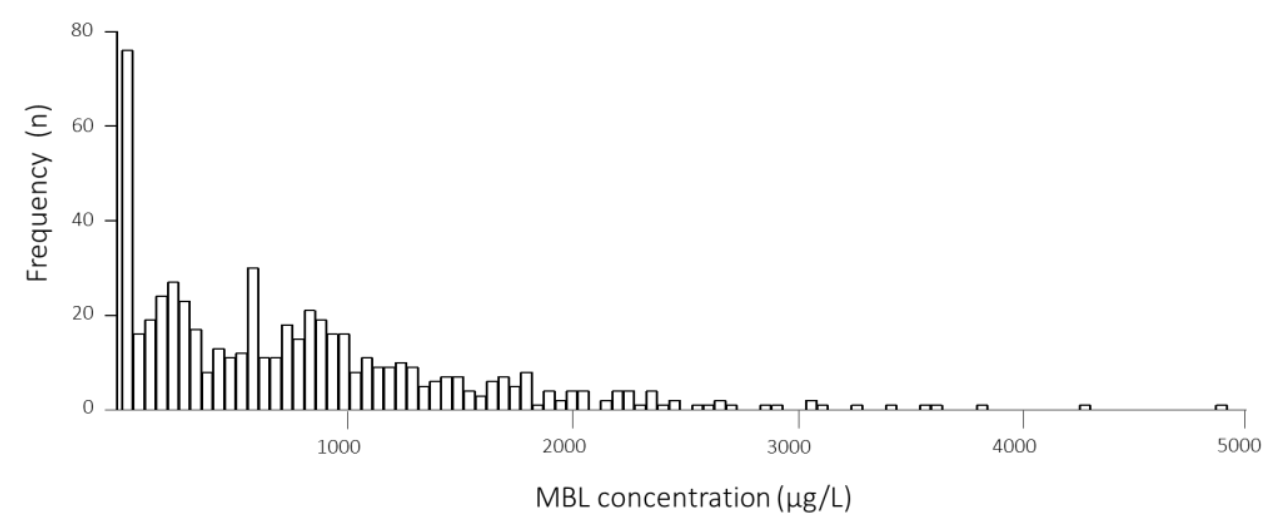

Figure 2: Distribution of plasma MBL. 
Regression analyses: longitudinal associations of lectin pathway factors with inflammation, endothelial dysfunction or cIMT were investigated with generalized estimating equations (GEE) using an exchangeable correlation structure. Associations of lectin pathway factors with incident CVD and CVE were investigated with logistic regression.

Based on previous studies that suggested a U-shaped association with cardiovascular outcomes such as with cIMT [17], the second tertile of MBL was chosen as reference category. In the analyses of $\mathrm{MBL}$ genotypes, the wildtype allele (A/A) was chosen as reference, and variant genotypes $\mathrm{A} / 0$ and $0 / 0$ were pooled into one group of individuals with diminished plasma MBL because the group of participants with the 0/0-genotype ( $N=31$ ) was too small to be analysed separately. In analyses of MASP-1, MASP-2, MASP-3 and MAp44, first non-linearity of any potential relationship was examined by investigating associations of tertiles with the respective outcomes. Because no evidence for non-linearity was found, MASPs and MAp44 were analysed as continuous variables. To allow for a direct comparison of their effect sizes, MASPs and MAp44 were subsequently converted to their respective Z-score. Consequently, in all analyses the associations are presented per 1 SD increase in MASP-1, MASP-2, MASP-3 or MAp44.

Analyses were first adjusted for variables related to the inclusion procedure, which incorporated stratified sampling for age and sex, and oversampling of impaired glucose metabolism and T2DM (model 1, age [years]; sex [men/women]; impaired glucose metabolism [yes/no]; T2DM [yes/no]). Subsequently, analyses were adjusted for the following potentially confounding cardiovascular risk factors: waist, systolic blood pressure, total cholesterol, HDL-cholesterol, triacylglycerols, eGFR, smoking, physical activity and use of anti-hypertensive, lipid-modifying, anti-thrombotic or glucose-lowering medication (model 2, model 1 + waist $[\mathrm{cm}]$; systolic blood pressure $[\mathrm{mmHg}]$; total cholesterol $[\mathrm{mmol} / \mathrm{L}]$; $\mathrm{HDL}$-cholesterol [mmol/L]; triacylglycerides [mmol/L]; eGFR $\left[\mathrm{ml} \bullet 1.73 \mathrm{~m}^{2} \bullet\left(\mathrm{min}^{-1}\right)\right]$; current smoking [yes/no], ex-smoking [yes/no], smoking [pack years], meeting the Dutch recommendation for habitual physical activity [yes/no] [41]; use of lipid-modifying medication [yes/no]; use of glucose-lowering medication [yes/no]; use of anti-hypertensive medication [yes/no]; use of anti-thrombotic medication [yes/no]). All adjustments were done with the baseline value of confounders, and all GEE models were additionally adjusted for time of examination (follow-up [yes/no]) and follow-up time [years].

We also tested if the association of lectin pathway factors with inflammation, endothelial dysfunction or cIMT changed over time by including an interaction term between time and lectin pathway factors in the fully adjusted GEE models. In case of significant interaction with time, GEE analyses were stratified according to baseline and follow-up examination. Such stratified analyses are comparable to cross-sectional analyses (baseline lectin pathway factors are investigated for their relationship with cardiovascular outcomes at baseline) and prospective analyses (baseline lectin pathway factors are investigated for their relationship with cardiovascular outcomes at follow-up). Furthermore, all analyses were examined for effect modification by sex by including interaction terms between sex and $M B L$ tertiles, $M B L$ genotypes, MASP-1, MASP-2, MASP-3 or MAp44 in the model. If the interaction term was significant, separate analyses among men and women were conducted. 
Sensitivity analyses: all analyses were repeated with exclusion of participants with a possible acute inflammation (serum CRP concentrations $>10 \mathrm{mg} / \mathrm{L}, \mathrm{n}=38$ at baseline and $\mathrm{n}=42$ at follow-up). Furthermore, complete case analyses were performed. The results were comparable in all analyses; therefore only results obtained with the imputed data sets are presented. Finally, significant associations of lectin pathway factors with cardiovascular outcomes were additionally adjusted for other lectin pathway factors that showed significant associations with the respective outcome.

Assumptions for GEE and logistic regression analyses were met. Statistical analyses were performed using the SPSS package version 22.0 (SPSS, Chicago, IL, USA) and statistical significance was set at $\mathrm{P}<0.05$.

\section{Results}

\subsection{Study population}

574 participants were included and extensively phenotyped. After a median follow-up of 7.0 years [interquartile range (IQR) 6.9-7.1 years], 495 individuals participated in follow-up measurements (for details see flow-chart, figure 1). Table 1 shows baseline characteristics of the study population as a whole. At baseline, 28\% had CVD and 16\% had CVE. In supplemental table 1, baseline characteristics across tertiles of MBL, MASP-1, MASP-2, MASP-3 and MAp44 and according to $\mathrm{MBL}$ genotypes, respectively, are provided. The frequencies of the $\mathrm{MBL}$ genotype were $62 \% \mathrm{~A} / \mathrm{A}$ (=wildtype), $33 \% \mathrm{~A} / 0$ and $5 \% 00$. As the $\mathrm{A} / \mathrm{A}$ individuals were previously described to have highest $M B L$ levels whereas both the $A / 0$ and 00 individuals have lower $\mathrm{MBL}$ levels, the $\mathrm{A} / 0$ and 00 individuals were combined into one group for the regression analyses.

Over the 7-year follow-up period, the 459 participants with complete follow-up data showed an increase in the inflammation score from $-0.16 \pm 0.90$ to $0.10 \pm 1.05$, an increase in the endothelial dysfunction score from $-0.12 \pm 0.94$ to $0.08 \pm 1.04$, and an increase in cIMT from $768 \pm 157 \mu \mathrm{m}$ to $818 \pm 101 \mu \mathrm{m}$.

Of the 342 individuals without CVD at baseline, 73 developed CVD (21\%), of whom 39 experienced an event (CVE, 11\%) during follow-up. Individuals who experienced incident CVD had more often T2DM at baseline, and were characterized by higher inflammation, higher endothelial dysfunction, and a higher cIMT as compared to those who remained free of CVD during follow-up (supplemental table 2). 
Table 1: Baseline characteristics of the study population

$\mathrm{MBL}(\mu \mathrm{g} / \mathrm{L})$

MBL genotypes, $\mathrm{A} / 0$ and $0 / 0$ combined (\%)

MASP-1 $(\mu \mathrm{g} / \mathrm{L})$

MASP-2 $(\mu \mathrm{g} / \mathrm{L})$

MASP-3 $(\mu \mathrm{g} / \mathrm{L})$

MAp44 ( $\mu \mathrm{g} / \mathrm{L})$

\begin{tabular}{c} 
Total study population \\
$\mathrm{N}=567^{*}$ \\
\hline $614[187-1088]$ \\
$38 \%$ \\
$9140 \pm 3112$ \\
$1230 \pm 608$ \\
$7161 \pm 2338$ \\
$2441 \pm 562$
\end{tabular}

Age (years)

Sex (\%men)

Normal/impaired glucose metabolism/T2DM (\%)

$59.6 \pm 7.0$

61

HbA1c (\%)

$52 / 22 / 26$

$6.0 \pm 0.8$

HOMA2-IR

Waist (cm)

$1.61[1.11-2.54]$

$99 \pm 12$

Systolic blood pressure $(\mathrm{mmHg})$

Diastolic blood pressure $(\mathrm{mmHg})$

$140 \pm 19$

$82 \pm 9$

Fasting triacylglycerols ( $\mathrm{mmol} / \mathrm{L}$ )

$1.4[1.0-2.2]$

Fasting cholesterol $(\mathrm{mmol} / \mathrm{L})$

$5.2 \pm 1.0$

$1.2 \pm 0.3$

Fasting HDL cholesterol $(\mathrm{mmol} / \mathrm{L})$

$3.3 \pm 0.9$

Fasting LDL cholesterol ( $\mathrm{mmol} / \mathrm{L})$

$94[83-106]$

Estimated glomerular filtration rate $(\mathrm{ml} / \mathrm{min} / 1.73 \mathrm{~cm} 2)$

62

Current smokers/former smokers (\%)

$20 / 51$

Pack years of smoking in ex/current smokers

$23[12-36]$

Use of anti-hypertensive medication (\%)

38

Use of glucose-lowering medication (\%)

13

19

Use of lipid-modifying medication (\%)

21

Use of anti-thrombotic medication (\%)

$2.07[0.97-3.92]$

$\mathrm{CRP}(\mathrm{mg} / \mathrm{L})$

$1.55[1.13-2.27]$

Interleukin-6 (ng/L)

Interleukin-8 (ng/L)

$4.40[3.60-5.56]$

$6.21[5.26-7.52]$

$0.21[0.19-0.24]$

Soluble intercellular adhesion molecule-1 $(\mu \mathrm{g} / \mathrm{L})$

$1.42[0.99-2.26]$

Serum amyloid $\mathrm{A}(\mathrm{mg} / \mathrm{L})$

Von Willebrand factor (\%)

$119[91-161]$

Soluble E-selectin $(\mu \mathrm{g} / \mathrm{L})$

$8.16[5.98-10.40]$

Soluble vascular cell adhesion molecule-1 ( $\mu \mathrm{g} / \mathrm{L})$

332 [293-379]

cIMT $(\mu \mathrm{m})$

$774 \pm 157$

Prevalent CVD (\%)

28

Prevalent CVE (\%)

16

Data are presented as mean \pm standard deviation, median [interquartile range] or proportion.

* data before multiple imputation were available in $\mathrm{N}=533$ to 567 participants. 


\subsection{Associations of MBL with low-grade inflammation and endothelial dysfunction}

In the association of plasma MBL with the low-grade inflammation score, deviations from linearity were observed. Over the 7-year follow-up period, the inflammation score was lowest in participants in the middle tertile ( $T_{\text {Middle }}$ ) of MBL (table 2 ). In a model adjusted for age, sex, impaired glucose metabolism and T2DM, the inflammation score was in the lowest

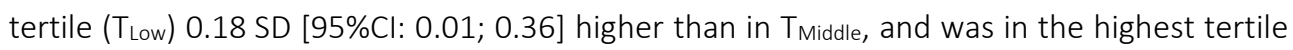
( $T_{\text {High }}$ ) 0.18 SD [0.02; 0.36] higher than in TMiddle. After adjustment for potential confounders, the difference between TLow and TMiddle remained significant (model $2, \beta=0.19$ SD [0.03; 0.34]) and the difference between $T_{H i g h}$ and $T_{\text {Middle }}$ became borderline significant ( $\beta=0.15$ SD [-0.02; $0.31]$ ). This suggested a U-shaped relationship between plasma MBL and the inflammation score. When participants with a potential acute inflammation (C-reactive protein (CRP) >10 $\mathrm{mg} / \mathrm{L}, \mathrm{n}=38$ at baseline, $\mathrm{n}=42$ at follow-up) were excluded from the analyses, these associations were strengthened: the inflammation score in TLow was 0.25 SD [0.09; 0.49] higher than in TMiddle, and was in THigh 0.20 SD [0.03; 0.36] higher than in TMiddle.

In contrast, plasma MBL was not associated with the endothelial dysfunction score in either model 1 or model 2 (table 2). Associations were unchanged when participants with a potential acute inflammation (CRP $>10 \mathrm{mg} / \mathrm{L}$ ) were excluded.

There was no interaction between $\mathrm{MBL}$ and time in these generalized estimating equations (GEE) models, and associations were comparable in men and women ( $P$-values for interaction $>0.05$ ).

\subsection{Associations of MBL with cIMT, incident CVD and incident CVE}

In the association between plasma MBL and CIMT, deviations from linearity were again observed. There was no difference in cIMT between $\mathrm{T}_{\text {Low }}$ and $\mathrm{T}_{\text {Middle. }}$ Participants in $\mathrm{T}_{\text {High }}$ had the lowest cIMT (table 2). In the fully adjusted model, cIMT was $28 \mu \mathrm{m}[-50 ;-5]$ ) lower in $\mathrm{T}_{\text {High }}$

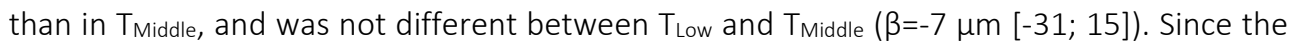
interaction term between $T_{H i g h}$ and time was significant $(P=0.044)$, associations of $M B L$ tertiles with cIMT were analysed separately for baseline and follow-up. In these stratified analyses, the difference in cIMT between $\mathrm{TH}_{\text {High }}$ and the lower tertiles in MBL (measured only at baseline) was more pronounced at baseline than at follow-up. In the fully adjusted model, baseline cIMT was $44 \mu \mathrm{m}[-73 ;-14]$ lower in $T_{\text {High }}$ than in $T_{\text {Middle }}$ of baseline MBL, while cIMT at follow-up was $7 \mu \mathrm{m}[-29 ; 14]$ lower in $T_{\text {High }}$ than in $\mathrm{T}_{\text {Middle }}$ of baseline MBL, indicating a cross-sectional but no prospective association. When participants with a potential acute inflammation (CRP>10 mg/L) were excluded from the analyses, the difference between $T_{\text {High }}$ and $T_{\text {Middle }}$ in CIMT was strengthened, both in the unstratified GEE association ( $\beta=-34 \mu \mathrm{m}$ [$58 ;-11])$ as well as in the cross-sectional association $(\beta=-52 \mu \mathrm{m}[-83 ;-22])$. Associations were comparable in men and women ( $P$-values for interaction $>0.05$ ).

In the associations with incident CVD, $\mathrm{T}_{\text {Low }}$ of baseline MBL had the highest and $\mathrm{T}_{\text {High }}$ had the lowest incidence of CVD (table 3). The difference between $T_{\text {High }}$ and $T_{\text {Middle }}$ was borderline 


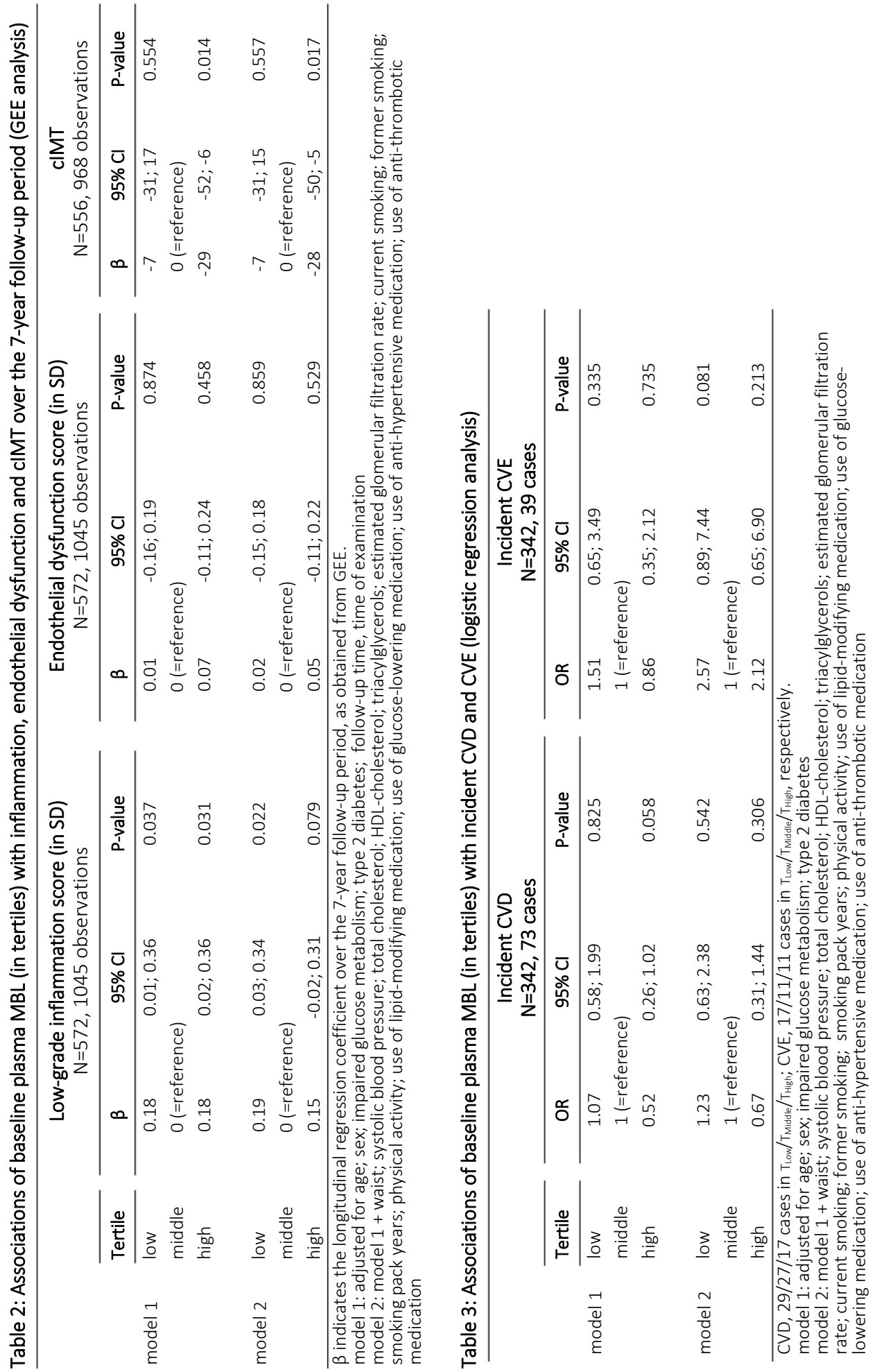


significant in a model adjusted for age, sex, impaired glucose metabolism and T2DM (model $1, \mathrm{OR}=0.52[0.26 ; 1.02]$ ). In the fully adjusted model, there were, however, no significant differences in CVD incidence between the tertiles. In the association with incident $C V D$, the interaction term between $T_{\text {Low }}$ of $M B L$ and sex was significant $(P=0.042)$. In analyses stratified for sex, however, no significant associations between MBL tertiles and CVD were observed in either men or women (model 2, men, $\mathrm{T}_{\text {Low }}$ compared to $\mathrm{T}_{\text {Middle: }} \mathrm{OR}=2.19$ [0.87; 5.52], $T_{\text {High }}$ compared to $T_{\text {Middle: }} \mathrm{OR}=0.86$ [0.31; 2.44]; women, $T_{\text {Low }}$ compared to $T_{\text {Middle: }}$ : $\mathrm{OR}=0.56[0.18 ; 1.80], \mathrm{T}_{\text {High }}$ compared to $\left.\mathrm{T}_{\text {Middle: }} \mathrm{OR}=0.81[0.23 ; 0.87]\right)$. In the associations with incident CVE, TLow again had the highest incidence of CVE (table 3). There were, however, no significant differences in CVE incidence between the tertiles. Associations with incident CVD and CVE were unchanged when participants with a potential acute inflammation (CRP $>10$ $\mathrm{mg} / \mathrm{L}$ ) were excluded. Because previous studies on MBL and CVD often contrasted two extremes in plasma MBL, we also performed an analysis using TLow as reference. In the fully adjusted model, the OR of THigh compared to TLow for CVD was 0.58 [0.29; 1.18], and the OR for CVE was 0.86 [0.33; 2.21].

In addition to the actual MBL plasma concentrations, we also investigated whether the genotypes coding for intermediate and low plasma MBL (the A/0 and 0/0 individuals) were associated with incident CVD or CVE. Participants with genotypes coding for lower plasma MBL had a slightly higher incidence of CVD (24\% in combined $A / 0$ and $0 / 0,20 \%$ in $A / A)$ and also CVE (15\% in combined $A / 0$ and $0 / 0,10 \%$ in $A / A)$, but this difference was not significant (model 2, combined $A / 0$ and $0 / 0$ compared to $A / A, C V D$ : OR=1.20 [0.68; 2.11]; CVE, OR=1.60 $[0.73 ; 3.51]$ ). Associations were comparable in men and women ( $P$-values for interaction $>0.05$ )

\subsection{Associations of MASP-1, MASP-2, MASP-3 and MAp44 with low-grade inflammation and endothelial dysfunction}

We did not observe deviations from linearity in associations of MASPs or MAp44 with the low-grade inflammation score or the endothelial dysfunction score, therefore MASPs and MAp44 were analysed as continuous variables.

MASP-1, MASP-2, MASP-3 and MAp44 were not associated with the inflammation score in the fully adjusted model (table 4). There was no interaction between MASPs or MAp44 and time in these GEE models.

MASP-1 and MASP-2 were not associated with the endothelial dysfunction score. In contrast, MASP-3 and MAp44 were positively associated with the endothelial dysfunction score (table 4). In the fully adjusted model (model 2), 1 SD higher baseline MASP-3 was associated with 0.10 SD $[0.02 ; 0.18]$ higher endothelial dysfunction score. Regarding MAp44, 1 SD higher baseline MAp44 was associated with 0.12 SD [0.04; 0.20] higher endothelial dysfunction score. Since the interaction term between MASP-3 and time was significant in the association with the endothelial dysfunction score ( $P$ for interaction=0.049), baseline and follow-up examinations were analysed separately. In this stratified analysis, the positive association between MASP-3 (measured only at baseline) with the baseline endothelial dysfunction 


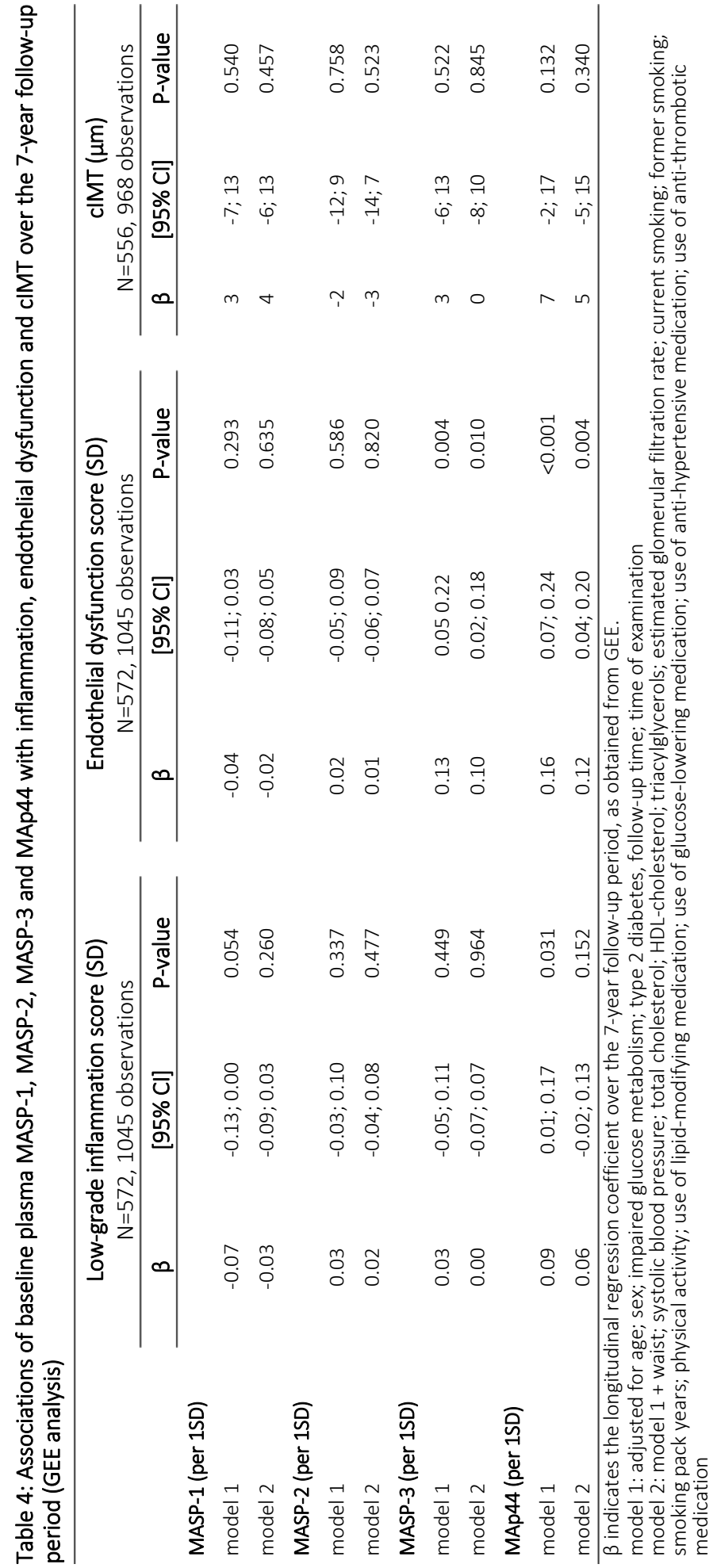


score was slightly stronger than with the endothelial dysfunction score at follow-up (baseline, $\beta=0.12$ SD [0.05; 0.19]; follow-up, $\beta=0.08$ SD [-0.02; 0.18]).

These associations were comparable in men and women ( $P$-values for interaction $>0.05$ ), and were unchanged when participants with a potential acute inflammation (CRP>10 mg/L) were excluded.

\subsection{Associations of MASP-1, MASP-2, MASP-3 and MAp44 with cIMT, incident CVD and incident CVE}

We did not observe deviations from linearity in associations of MASPs or MAp44 with cIMT, incident CVD or incident CVE. MASP-1, MASP-2, MASP-3 and MAp44 were not associated with cIMT in either model 1 or model 2 (table 4). There was no interaction between MASP1, MASP-2 or MASP-3 and time in any of these GEE models. Since the interaction term between MAp44 and time was significant in the association with cIMT ( $P$ for interaction<0.001), baseline and follow-up examinations were analysed separately. In this stratified analysis, however, no significant associations between baseline MAp44 and cIMT at either baseline or follow-up were observed (model 2, baseline: $\beta=11 \mu \mathrm{m}[-2 ; 34]$; followup: $\beta=-5 \mu \mathrm{m}[-15 ; 6])$. There were no associations between MASP-1, MASP-2, MASP-3 or MAp44 with incident CVD or CVE (table 5).

These associations were comparable in men and women ( $P$-values for interaction $>0.05$ ), and were unchanged when participants with a potential acute inflammation (CRP>10 mg/L) were excluded.

Table 5: Associations of baseline plasma MASP-1, MASP-2, MASP-3 and MAp44 with incident CVD and CVE (logistic regression analysis)

\begin{tabular}{|c|c|c|c|c|c|c|}
\hline & \multicolumn{3}{|c|}{$\begin{array}{c}\text { Incident CVD } \\
\mathrm{N}=342,73 \text { cases }\end{array}$} & \multicolumn{3}{|c|}{$\begin{array}{c}\text { Incident CVE } \\
\mathrm{N}=342,39 \text { cases }\end{array}$} \\
\hline & OR & {$[95 \% \mathrm{Cl}]$} & P-value & OR & {$[95 \% \mathrm{Cl}]$} & P-value \\
\hline \multicolumn{7}{|c|}{ MASP-1 (per 1SD) } \\
\hline model 1 & 0.84 & $0.63 ; 1.12$ & 0.232 & 0.73 & $0.49 ; 1.07$ & 0.107 \\
\hline model 2 & 0.83 & $0.62 ; 1.13$ & 0.241 & 0.70 & $0.43 ; 1.13$ & 0.139 \\
\hline \multicolumn{7}{|c|}{ MASP-2 (per 1SD) } \\
\hline model 1 & 0.91 & $0.70 ; 1.18$ & 0.475 & 0.95 & $0.68 ; 1.32$ & 0.757 \\
\hline model 2 & 0.92 & $0.69 ; 1.23$ & 0.574 & 1.00 & $0.67 ; 1.50$ & 0.999 \\
\hline \multicolumn{7}{|c|}{ MASP-3 (per 1SD) } \\
\hline model 1 & 1.21 & $0.95 ; 1.54$ & 0.121 & 1.14 & $0.85 ; 1.53$ & 0.376 \\
\hline model 2 & 1.23 & $0.95 ; 1.59$ & 0.121 & 1.20 & $0.85 ; 1.69$ & 0.291 \\
\hline \multicolumn{7}{|c|}{ MAp44 (per 1SD) } \\
\hline model 1 & 0.99 & $0.74 ; 1.32$ & 0.918 & 0.88 & $0.60 ; 1.29$ & 0.507 \\
\hline model 2 & 0.97 & $0.70 ; 1.35$ & 0.857 & 0.72 & $0.44 ; 1.19$ & 0.200 \\
\hline
\end{tabular}

model 1: adjusted for age; sex; impaired glucose metabolism; type 2 diabetes

model 2: model 1 + waist; systolic blood pressure; total cholesterol; HDL-cholesterol; triacylglycerols; estimated glomerular filtration rate; current smoking; former smoking; smoking pack years; physical activity; use of lipidmodifying medication; use of glucose-lowering medication; use of anti-hypertensive medication; use of antithrombotic medication 


\subsection{Sensitivity analyses}

When we mutually adjusted the respective associations of plasma MBL, MASP-3 and MAp44 with cardiovascular outcomes for one another, these associations were not materially altered. Regarding the association between $\mathrm{MBL}$ and the inflammation score, upon additional adjustment for MASP-3 and MAp44, TLow of plasma MBL was 0.19 SD [0.03; 0.34]

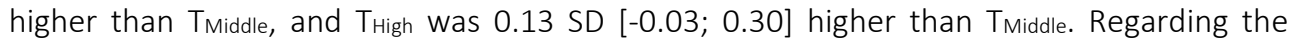
association between MBL and CIMT, upon additional adjustment for MASP-3 and MAp44, $T_{\text {High }}$ of plasma MBL was $29 \mu \mathrm{m}[-52 ;-6]$ lower than TMiddle. Regarding the association between MASP-3 and the endothelial dysfunction score, upon additional adjustment for MBL tertiles and MAp44, $\beta$ was 0.08 SD [0.01; 0.16]. Regarding the association of MAp44 with the endothelial dysfunction score, upon additional adjustment for MBL tertiles and MASP-3, $\beta$ was 0.10 SD [0.02; 0.18].

\section{Discussion}

The present comprehensive evaluation of initiating factors of the lectin pathway of complement activation in relation to development of CVD had several main findings: with respect to tertiles of plasma MBL, we observed that both high and low MBL was associated with low-grade inflammation, but that MBL was not associated with endothelial dysfunction. Furthermore, high plasma MBL was associated with lower cIMT, but was not significantly associated with CVD or CVE. Associations of MBL genotypes with CVD were similar to those of plasma MBL, and were also not significant. With respect to MASPs and MAp44, we observed that increasing concentrations of MASP-3 and MAp44 but not MASP-1 or MASP-2 were associated with augmented endothelial dysfunction. MASP-1, MASP-2, MASP-3 and MAp44 were not associated with low-grade inflammation or CIMT, nor with incident CVD or CVE.

These findings substantially extend previous knowledge. In previous studies, unadjusted correlations between plasma MBL and a single inflammatory marker, such as CRP, were investigated [5, 10, 12, 13, 47]. Mostly no correlation between MBL and inflammatory markers was found, but these studies did not consider a potential non-linear relationship. This is the first large, prospective study on MBL and cIMT. Previous studies on CIMT were cross-sectional, substantially smaller, and were done in selected populations $[17,48]$. We observed that high plasma MBL may be beneficial in atherosclerosis as reflected by CIMT, while both high and low MBL may be adverse with regard to low-grade inflammation. This suggests that MBL may indeed have protective as well as harmful effects in the vasculature, which explains why previous large studies on MBL and incident CVD partly had contradictory findings. This is also the first study on the role of MASPs and MAp44 in development of CVD. The few previous studies on MASPs in CVD were case-control studies [20], or measured MASPs at admission for an acute cardiovascular event [21], or measured only a single MASP (MASP-2) [22].

Over the 7-year follow-up period, the low and high MBL tertiles were associated with more inflammation than the middle tertile. A dual role of $\mathrm{MBL}$ in inflammation (i.e. beneficial 
effects for anti-microbial defence and adverse effects as an initiator of auto-inflammatory mechanisms) may be explained by impaired clearance processes upon low MBL, while high $\mathrm{MBL}$ may result in hyperresponsiveness to inflammatory triggers. Low MBL was shown to compromise the immune response and to limit the capacity to remove waste products such as apoptotic cells [9]. Consequently, low MBL may entail more inflammation as a result of chronic infections and impaired turnover of dysfunctional tissues. On the other hand, high $\mathrm{MBL}$ may result in hyperalertness of the lectin pathway and may increase inflammation as a result of oxidative stress or membrane damage. This is suggested by studies in ischemiareperfusion injury, rheumatoid arthritis or diabetic nephropathy, which showed that high MBL was associated with cardiovascular and renal damage [11, 14, 15, 49, 50]. We observed that this $U$-shaped association of MBL with inflammation was considerably strengthened upon exclusion of participants with elevated CRP concentrations. Although MBL is only a weak acute phase-reactant $[51,52]$, analyses that limit the influence of acute inflammation are more likely to reflect the relationship of MBL with chronic low-grade inflammation. The fact that MBL was not associated with endothelial dysfunction suggests that associations of low MBL with low-grade inflammation and atherosclerosis may be explained by a crucial role of $\mathrm{MBL}$ in clear-up processes inside the vessel wall, but not by effects on endothelial activation or damage.

Our observations on MBL and CIMT, CVD and CVE are in line with the hypothesized beneficial effects of MBL in vascular clear-up processes. Participants with high plasma MBL had lower CIMT and were also less likely to develop CVD and CVE, although the latter associations were non-significant. Associations of MBL genotypes with CVD and CVE were also non-significant, but showed similar patterns as observed in large studies in comparable populations. At first sight, our results for cIMT are in contrast with the previously largest study on cIMT ( $N=114)$, which reported a U-shaped association between MBL concentrations and cIMT [17]. Notably, in that study on patients with rheumatoid arthritis, MBL concentrations were up to twice as high as in our study. In line with our findings, a smaller study in systematic lupus erythematosus patients with MBL concentrations comparable to our study observed that high plasma MBL was indeed associated with lower cIMT [48]. Beneficial effects of high MBL in the vessel wall despite higher systemic inflammation may be explained by the opsonic activity of MBL, potentially independent of downstream complement activation [8, 9].

Associations of MASP-1, MASP-2, MASP-3 and MAp44 with cardiovascular outcomes differed from those of MBL. Over the 7-year follow-up, MASP-3 and MAp44 were positively associated with endothelial dysfunction, but not with low-grade inflammation. MASP-1 and MASP-2 were neither associated with low-grade inflammation nor with endothelial dysfunction. Biological functions of MASP-3 and MAp44 are hitherto unclear. In contrast to the proteases MASP-1 and MASP-2, for whom the enzymatic activities are well described, much less is known for MASP-3. MASP-3 may not primarily be involved in the activation of any downstream complement proteases [18, 19], but may rather be involved in developmental processes $[53,54]$. MAp44 has no catalytic activity at all, and is thought to exert biological functions only via binding to other proteins [18, 19, 25]. An effect of MAp44 is thus likely to involve a regulatory function on the activities of the three MASPs, i.e. by 
competing for binding to MBL. Notably, MBL, MASP-1 and MASP-2 were not associated with endothelial dysfunction, and associations of MASP-3 and MAp44 with endothelial dysfunction were independent of plasma MBL. This opens up the possibility that these associations may be explained by effects beyond lectin pathway activation. So far, the only identified substrate for MASP-3 is insulin-like growth factor binding protein-5 [55], which regulates the activity of insulin-like growth factor. Insulin-like growth factor is increasingly implicated in endothelial function [56], which might potentially explain our observations. Future research may clarify the functions of MASP-3 and MAp44 in lectin pathway regulation, and may identify further, potentially non-complement substrates of MASP-3. Those insights are needed to understand how MASP-3 and MAp44 may contribute to endothelial dysfunction and indeed to basic tissue developmental processes. There are other proteins, i.e. the three ficolins and the collectin CL-LK that interact with MASP-3 and the other MASPS and MAp44 [2]. Whether the effect of MASP-3 and MAp44 is mediated via interactions with these other molecules remains to be examined in future studies.

We did not observe associations of MASP-1, MASP-2, MASP-3 or MAp44 with cIMT, incident CVD or CVE. This was in line with a previous cross-sectional study on cases with coronary artery disease and controls [20]. We did observe a positive, but non-significant OR of MASP3 for CVD and CVE. Given the relatively low power to detect CVD and CVE in our study and the observed longitudinal association of MASP-3 with endothelial dysfunction, this observation might potentially be biologically relevant, but this needs to be confirmed in larger prospective studies. Overall, plasma concentrations of MASPs and MAp44 do not appear to reflect any major contribution to cardiovascular risk.

Our study has several strengths but also limitations. The greatest strength is the concomitant measurement of MBL, MASP-1, MASP-2, MASP-3 and MAp44 in a large, extensively phenotyped cohort. The detailed phenotyping of the participants allowed us to investigate lectin pathway factors in low-grade inflammation, endothelial dysfunction and atherosclerosis in order to gain an insight into the role of the lectin pathway in different pathophysiological aspects of CVD. Additionally, our study was also large enough to investigate potential non-linear associations. The most relevant limitation of our study is its observational nature. Furthermore, our study cohort was selected for a moderately increased risk of cardiometabolic disease, which might limit the generalizability of our results. Notwithstanding, our cohort represents a large part of typical Western populations, namely middle-aged to elderly individuals that are overweight, modestly hypertensive, have some signs of a disturbed glucose metabolism and/or use medication for various reasons.

In conclusion, our findings indicate that MBL may be protective in atherosclerosis but may have a dual role in low-grade inflammation. Low MBL may result in impaired clear-up processes, which in turn may induce inflammation and favour the development of atherosclerosis. High MBL may render the lectin pathway hyperresponsive to inflammatory triggers. Proteases MASP-1 and MASP-2, which induce further complement activation upon $\mathrm{MBL}$-binding to ligands, were not associated with cardiovascular outcomes. This suggests that the capacity to recognize targets via MBL rather than the efficiency of MASP-1 and MASP-2-mediated complement activation is relevant for the role of the lectin pathway in 
inflammation and atherosclerosis. MASP-3 and MAp44 were positively associated with endothelial dysfunction. Given that MBL itself was not associated with endothelial dysfunction, this suggests that MASP-3 and MAp44 may potentially have functions in endothelial activation independent of lectin-pathway activation. In figure 3, our observations on factors of the lectin pathway are summarized in a graphical representation.

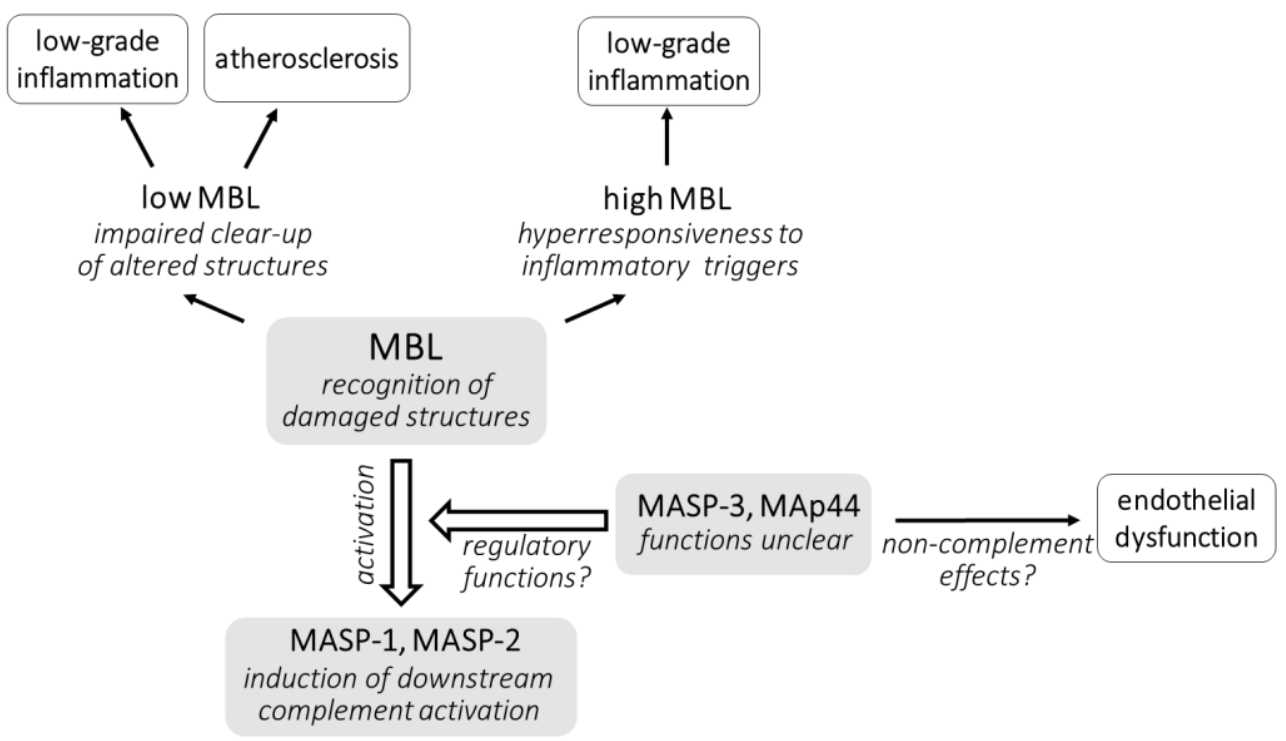

Figure 3: Schematic representation of the observed associations and proposed underlying mechanisms. 


\section{References}

1. Pagowska-Klimek I, Cedzynski M. Mannan-binding lectin in cardiovascular disease. Biomed Res. Int., 2014. 2014: 616817.

2. Degn SE, Thiel S. Humoral pattern recognition and the complement system. Scand. J. Immunol., 2013. 78: 18193.

3. Best LG, Davidson M, North KE, MacCluer JW, Zhang Y, Lee ET, Howard BV, DeCroo S, Ferrell RE. Prospective analysis of mannose-binding lectin genotypes and coronary artery disease in American Indians: the Strong Heart Study. Circulation, 2004. 109: 471-5.

4. Dahl M, Tybjaerg-Hansen A, Schnohr P, Nordestgaard BG. A population-based study of morbidity and mortality in mannose-binding lectin deficiency. J. Exp. Med., 2004. 199: 1391-9.

5. Siezenga MA, Chandie Shaw PK, Daha MR, Rabelink TJ, Berger SP. Low Mannose-Binding Lectin (MBL) genotype is associated with future cardiovascular events in type 2 diabetic South Asians A prospective cohort study. Cardiovasc. Diabetol., 2011. 10: 60.

6. Vengen IT, Madsen HO, Garred P, Platou C, Vatten L, Videm V. Mannose-binding lectin deficiency is associated with myocardial infarction: the HUNT2 study in Norway. PLoS ONE, 2012. 7: e42113.

7. Rugonfalvi-Kiss S, Endresz V, Madsen HO, Burian K, Duba J, Prohaszka Z, Karadi I, Romics L, Gonczol E, Fust G, Garred P. Association of Chlamydia pneumoniae with coronary artery disease and its progression is dependent on the modifying effect of mannose-binding lectin. Circulation, 2002. 106: 1071-6.

8. Takahashi K. Mannose-binding lectin and the balance between immune protection and complication. Expert Rev. Anti Infect. Ther., 2011. 9: 1179-90.

9. Ip WK, Takahashi K, Ezekowitz RA, Stuart LM. Mannose-binding lectin and innate immunity. Immunol. Rev., 2009. 230: 9-21.

10. Keller TT, van Leuven SI, Meuwese MC, Wareham NJ, Luben R, Stroes ES, Hack CE, Levi M, Khaw KT, Boekholdt SM. Serum levels of mannose-binding lectin and the risk of future coronary artery disease in apparently healthy men and women. Arterioscler. Thromb. Vasc. Biol., 2006. 26: 2345-50.

11. Troelsen LN, Garred P, Madsen HO, Jacobsen S. Genetically determined high serum levels of mannose-binding lectin and agalactosyl IgG are associated with ischemic heart disease in rheumatoid arthritis. Arthritis Rheum., 2007. 56: 21-9.

12. Hansen TK, Gall MA, Tarnow L, Thiel S, Stehouwer CD, Schalkwijk CG, Parving HH, Flyvbjerg A. Mannosebinding lectin and mortality in type 2 diabetes. Arch. Intern. Med., 2006. 166: 2007-13.

13. Saevarsdottir S, Oskarsson OO, Aspelund T, Eiriksdottir G, Vikingsdottir T, Gudnason V, Valdimarsson H. Mannan binding lectin as an adjunct to risk assessment for myocardial infarction in individuals with enhanced risk. J. Exp. Med., 2005. 201: 117-25.

14. Collard CD, Shernan SK, Fox AA, Bernig T, Chanock SJ, Vaughn WK, Takahashi K, Ezekowitz AB, Jarolim P, Body SC. The MBL2 'LYQA secretor' haplotype is an independent predictor of postoperative myocardial infarction in whites undergoing coronary artery bypass graft surgery. Circulation, 2007. 116: I106-12.

15. Trendelenburg $M$, Theroux $P$, Stebbins A, Granger $C$, Armstrong $P$, Pfisterer $M$. Influence of functional deficiency of complement mannose-binding lectin on outcome of patients with acute ST-elevation myocardial infarction undergoing primary percutaneous coronary intervention. Eur. Heart J., 2010. 31: 1181-7.

16. Rugonfalvi-Kiss S, Dosa E, Madsen HO, Endresz V, Prohaszka Z, Laki J, Karadi I, Gonczol E, Selmeci L, Romics L, Fust G, Entz L, Garred P. High rate of early restenosis after carotid eversion endarterectomy in homozygous carriers of the normal mannose-binding lectin genotype. Stroke, 2005. 36: 944-8.

17. Troelsen LN, Garred P, Christiansen B, Torp-Pedersen C, Christensen IJ, Narvestad E, Jacobsen S. Double role of mannose-binding lectin in relation to carotid intima-media thickness in patients with rheumatoid arthritis. Mol. Immunol., 2010. 47: 713-8

18. Yongqing T, Drentin N, Duncan RC, Wijeyewickrema LC, Pike RN. Mannose-binding lectin serine proteases and associated proteins of the lectin pathway of complement: two genes, five proteins and many functions? Biochim. Biophys. Acta, 2012. 1824: 253-62.

19. Matsushita M, Endo Y, Fujita T. Structural and functional overview of the lectin complement pathway: its molecular basis and physiological implication. Arch. Immunol. Ther. Exp. (Warsz.), 2013. 61: 273-83.

20. Frauenknecht V, Thiel S, Storm L, Meier N, Arnold M, Schmid JP, Saner H, Schroeder V. Plasma levels of mannan-binding lectin (MBL)-associated serine proteases (MASPs) and MBL-associated protein in cardio- and cerebrovascular diseases. Clin. Exp. Immunol., 2013. 173: 112-20.

21. Holt CB, Thiel S, Munk K, Ostergaard JA, Botker HE, Hansen TK. Association between endogenous complement inhibitor and myocardial salvage in patients with myocardial infarction. Eur. Heart J. Acute Cardiovas. Care, 2014. 3: 3-9.

22. Mellbin LG, Bjerre M, Thiel S, Hansen TK. Complement activation and prognosis in patients with type 2 diabetes and myocardial infarction: a report from the DIGAMI 2 trial. Diabetes Care, 2012. 35: 911-7. 
23. Kruijshoop M, Feskens EJ, Blaak EE, de Bruin TW. Validation of capillary glucose measurements to detect glucose intolerance or type 2 diabetes mellitus in the general population. Clin. Chim. Acta, 2004. 341: 33-40.

24. Hertle E, van Greevenbroek MM, Arts IC, van der Kallen CJ, Geijselaers SL, Feskens EJ, Jansen EH, Schalkwijk CG, Stehouwer CD. Distinct associations of complement C3a and its precursor C3 with atherosclerosis and cardiovascular disease. The CODAM study. Thromb. Haemost., 2014. 111: 1102-11.

25. Degn SE, Jensen L, Gal P, Dobo J, Holmvad SH, Jensenius JC, Thiel S. Biological variations of MASP-3 and MAp44, two splice products of the MASP1 gene involved in regulation of the complement system. J. Immunol. Methods, 2010. 361: 37-50.

26. Hansen TK, Tarnow L, Thiel S, Steffensen R, Stehouwer CD, Schalkwijk CG, Parving HH, Flyvbjerg A. Association between mannose-binding lectin and vascular complications in type 1 diabetes. Diabetes, 2004. 53: $1570-6$.

27. Hertle E, van Greevenbroek MM, Arts IC, van der Kallen CJ, Feskens EJ, Schalkwijk CG, Stehouwer CD. Complement activation products $\mathrm{C} 5 \mathrm{a}$ and $\mathrm{s} \mathrm{C} 5 \mathrm{~b}-9$ are associated with low-grade inflammation and endothelial dysfunction, but not with atherosclerosis in a cross-sectional analysis: the CODAM study. Int. J. Cardiol., 2014. 174: 400-3.

28. Kelly M, Semsarian C. Multiple mutations in genetic cardiovascular disease: a marker of disease severity? Circ. Cardiovasc. Genet., 2009. 2: 182-90.

29. Lundbo LF, Harboe ZB, Clausen LN, Hollegaard MV, Sorensen HT, Hougaard DM, Konradsen HB, Norgaard M, Benfield T. Mannose-binding lectin gene, MBL2, polymorphisms are not associated with susceptibility to invasive pneumococcal disease in children. Clin. Infect. Dis., 2014. 59: e66-71.

30. Mellbin LG, Hamsten A, Malmberg K, Steffensen R, Ryden L, Ohrvik J, Hansen TK. Mannose-binding lectin genotype and phenotype in patients with type 2 diabetes and myocardial infarction: a report from the DIGAMI 2 trial. Diabetes Care, 2010. 33: 2451-6.

31. Jacobs M, van Greevenbroek MM, van der Kallen CJ, Ferreira I, Blaak EE, Feskens EJ, Jansen EH, Schalkwijk CG, Stehouwer CD. Low-grade inflammation can partly explain the association between the metabolic syndrome and either coronary artery disease or severity of peripheral arterial disease: the CODAM study. Eur. J. Clin. Invest., 2009. 39: 437-44.

32. Jacobs M, van Greevenbroek MM, van der Kallen CJ, Ferreira I, Blaak EE, Feskens EJ, Jansen EH, Schalkwijk CG, Stehouwer CD. The association between the metabolic syndrome and peripheral, but not coronary, artery disease is partly mediated by endothelial dysfunction: the CODAM study. Eur. J. Clin. Invest., 2011. 41: 16775.

33. van Bussel BC, Ferreira I, van de Waarenburg MP, van Greevenbroek MM, van der Kallen CJ, Henry RM, Feskens EJ, Stehouwer CD, Schalkwijk CG. Multiple Inflammatory Biomarker Detection in a Prospective Cohort Study: A Cross-Validation between Well-Established Single-Biomarker Techniques and an Electrochemiluminescense-Based Multi-Array Platform. PLoS ONE, 2013. 8: e58576.

34. Stam F, van Guldener C, Schalkwijk CG, ter Wee PM, Donker AJ, Stehouwer CD. Impaired renal function is associated with markers of endothelial dysfunction and increased inflammatory activity. Nephrol. Dial. Transplant., 2003. 18: 892-898.

35. Engelen L, Ferreira I, Gaens KH, Henry RM, Dekker JM, Nijpels G, Heine RJ, t Hart LM, van Greevenbroek MM, van der Kallen CJ, Blaak EE, Feskens EJ, Ten Cate H, Stehouwer CD, Schalkwijk CG. The association between the -374T/A polymorphism of the receptor for advanced glycation endproducts gene and blood pressure and arterial stiffness is modified by glucose metabolism status: the Hoorn and CoDAM studies. J. Hypertens., 2010. 28: 285-93.

36. Aboyans V, Criqui MH, Abraham P, Allison MA, Creager MA, Diehm C, Fowkes FG, Hiatt WR, Jonsson B, Lacroix P, Marin B, McDermott MM, Norgren L, Pande RL, Preux PM, Stoffers HE, Treat-Jacobson D. Measurement and interpretation of the ankle-brachial index: a scientific statement from the American Heart Association. Circulation, 2012. 126: 2890-909.

37. Atsma F, Bartelink ML, Grobbee DE, van der Schouw YT. Best reproducibility of the ankle-arm index was calculated using Doppler and dividing highest ankle pressure by highest arm pressure. J. Clin. Epidemiol., 2005. 58: 1282-8.

38. Alberti KG, Zimmet PZ. Definition, diagnosis and classification of diabetes mellitus and its complications. Part 1: diagnosis and classification of diabetes mellitus provisional report of a WHO consultation. Diabet. Med., 1998. 15: 539-53.

39. Wlazlo N, van Greevenbroek MM, Ferreira I, Jansen EJ, Feskens EJ, van der Kallen CJ, Schalkwijk CG, Bravenboer B, Stehouwer CD. Low-grade inflammation and insulin resistance independently explain substantial parts of the association between body fat and serum C3: the CODAM study. Metabolism, 2012. 61: 1787-96.

40. Wendel-Vos GC, Schuit AJ, Saris WH, Kromhout D. Reproducibility and relative validity of the short questionnaire to assess health-enhancing physical activity. J. Clin. Epidemiol., 2003. 56: 1163-9.

41. Kemper HC, Ooijendijk WT, Stiggelbout M. Consensus over de Nederlandse Norm voor Gezond Bewegen. Tijdschr. Soc. Gezondheidsz., 2000. 78: 180-183. 
42. Levey AS, Bosch JP, Lewis JB, Greene T, Rogers N, Roth D. A more accurate method to estimate glomerular filtration rate from serum creatinine: a new prediction equation. Modification of Diet in Renal Disease Study Group. Ann. Intern. Med., 1999. 130: 461-70.

43. Friedewald WT, Levy RI, Fredrickson DS. Estimation of the concentration of low-density lipoprotein cholesterol in plasma, without use of the preparative ultracentrifuge. Clin. Chem., 1972. 18: 499-502.

44. Wlazlo N, van Greevenbroek MM, Ferreira I, Feskens EJ, van der Kallen CJ, Schalkwijk CG, Bravenboer B, Stehouwer CD. Complement factor 3 is associated with insulin resistance and with incident type 2 diabetes over a 7-year follow-up period: the CODAM Study. Diabetes Care, 2014. 37: 1900-9.

45. Sterne JA, White IR, Carlin JB, Spratt M, Royston P, Kenward MG, Wood AM, Carpenter JR. Multiple imputation for missing data in epidemiological and clinical research: potential and pitfalls. BMJ, 2009. 338: b2393.

46. van Bussel BC, Schouten F, Henry RM, Schalkwijk CG, de Boer MR, Ferreira I, Smulders YM, Twisk JW, Stehouwer CD. Endothelial dysfunction and low-grade inflammation are associated with greater arterial stiffness over a 6-year period. Hypertension, 2011. 58: 588-95.

47. Kildey K, Rooks K, Weier S, Flower RL, Dean MM. Effect of age, gender and mannose-binding lectin (MBL) status on the inflammatory profile in peripheral blood plasma of Australian blood donors. Hum. Immunol., 2014. 75: 973-9.

48. Troelsen LN, Garred P, Christiansen B, Torp-Pedersen C, Jacobsen S. Genetically determined serum levels of mannose-binding lectin correlate negatively with common carotid intima-media thickness in systemic lupus erythematosus. J. Rheumatol., 2010. 37: 1815-21.

49. Hansen TK, Forsblom C, Saraheimo M, Thorn L, Waden J, Hoyem P, Ostergaard J, Flyvbjerg A, Groop PH. Association between mannose-binding lectin, high-sensitivity C-reactive protein and the progression of diabetic nephropathy in type 1 diabetes. Diabetologia, 2010. 53: 1517-24.

50. Zhang N, Zhuang M, Ma A, Wang G, Cheng P, Yang Y, Wang X, Zhang J, Chen X, Lu M. Association of levels of mannose-binding lectin and the MBL2 gene with type 2 diabetes and diabetic nephropathy. PLoS ONE, 2013. 8: e83059.

51. Ytting H, Christensen IJ, Basse L, Lykke J, Thiel S, Jensenius JC, Nielsen HJ. Influence of major surgery on the mannan-binding lectin pathway of innate immunity. Clin. Exp. Immunol., 2006. 144: 239-46.

52. Dean MM, Minchinton RM, Heatley S, Eisen DP. Mannose binding lectin acute phase activity in patients with severe infection. J. Clin. Immunol., 2005. 25: 346-52.

53. Degn SE, Jensenius JC, Thiel S. Disease-causing mutations in genes of the complement system. Am. J. Hum. Genet., 2011. 88: 689-705.

54. Sirmaci A, Walsh T, Akay H, Spiliopoulos M, Sakalar YB, Hasanefendioglu-Bayrak A, Duman D, Farooq A, King MC, Tekin M. MASP1 mutations in patients with facial, umbilical, coccygeal, and auditory findings of Carnevale, Malpuech, OSA, and Michels syndromes. Am. J. Hum. Genet., 2010. 87: 679-86.

55. Cortesio CL, Jiang W. Mannan-binding lectin-associated serine protease 3 cleaves synthetic peptides and insulin-like growth factor-binding protein 5. Arch. Biochem. Biophys., 2006. 449: 164-70.

56. Bach LA. Endothelial cells and the IGF system. J. Mol. Endocrinol., 2015. 54: R1-13. 


\section{Supplemental data}

Supplemental table 1: Baseline characteristics of the study population according to tertiles of MBL, MASP1, MASP-2, MASP-3, MAp44 and according to MBL genotypes

a) According to tertiles of plasma MBL concentrations

\begin{tabular}{|c|c|c|c|}
\hline Baseline characteristics * & $\begin{array}{c}\text { Tertile } 1 \\
\text { n=189 }\end{array}$ & $\begin{array}{c}\text { Tertile } 2 \\
\text { n=189 }\end{array}$ & $\begin{array}{c}\text { Tertile } 3 \\
\mathrm{n}=189\end{array}$ \\
\hline $\mathrm{MBL}(\mu \mathrm{g} / \mathrm{L})$ & $86[0.1-188]$ & 614 [484-775] & 1408 [1086-1839] \\
\hline MBL genotypes, $\mathrm{A} / 0$ and $0 / 0$ combined (\%) & 84 & 24 & 4 \\
\hline MASP-1 ( $\mu \mathrm{g} / \mathrm{L})$ & $10460 \pm 2940$ & $9317 \pm 2889$ & $7614 \pm 2844$ \\
\hline MASP-2 ( $\mu \mathrm{g} / \mathrm{L})$ & $1482 \pm 660$ & $1182 \pm 538$ & $1024 \pm 538$ \\
\hline MASP-3 ( $\mu \mathrm{g} / \mathrm{L})$ & $7272 \pm 2325$ & $7009 \pm 2497$ & $7239 \pm 2201$ \\
\hline MAp44 ( $\mu \mathrm{g} / \mathrm{L})$ & $2420 \pm 596$ & $2407 \pm 574$ & $2492 \pm 515$ \\
\hline C3a $(\mu \mathrm{g} / \mathrm{L})$ & $61[50-72]$ & $58[48-72]$ & $58[50-71]$ \\
\hline $\mathrm{C} 5 \mathrm{a}(\mu \mathrm{g} / \mathrm{L})$ & $7.9 \pm 4.1$ & $7.3 \pm 3.6$ & $7.6 \pm 3.8$ \\
\hline sC5b-9 ( $\mu \mathrm{g} / \mathrm{L})$ & $113 \pm 35$ & $113 \pm 36$ & $114 \pm 33$ \\
\hline Age (years) & $59 \pm 7$ & $60 \pm 7$ & $59 \pm 7$ \\
\hline Sex $(\%$ men $)$ & 69 & 58 & 68 \\
\hline Normal/impaired glucose metabolism/T2DM (\%) & $51 / 20 / 29$ & $54 / 26 / 20$ & $50 / 21 / 29$ \\
\hline $\mathrm{HbA} 1 \mathrm{c}(\%)$ & $6.0 \pm 0.9$ & $5.9 \pm 0.7$ & $6.0 \pm 0.9$ \\
\hline HOMA2-IR & $1.51[1.01-2.38]$ & $1.63[1.12-2.67]$ & $1.70[1.16-2.56]$ \\
\hline Waist $(\mathrm{cm})$ & $100 \pm 12$ & $99 \pm 12$ & $99 \pm 12$ \\
\hline Systolic blood pressure (mmHg) & $140 \pm 18$ & $142 \pm 21$ & $139 \pm 19$ \\
\hline Diastolic blood pressure (mmHg) & $81 \pm 10$ & $83 \pm 9$ & $82 \pm 9$ \\
\hline Fasting triacylglycerols (mmol/L) & $1.50[1.00-2.00]$ & $1.40[1.00-1.95]$ & $1.30[0.90-2.05]$ \\
\hline Fasting cholesterol (mmol/L) & $5.3 \pm 1.1$ & $5.3 \pm 0.9$ & $5.1 \pm 0.9$ \\
\hline Fasting HDL cholesterol (mmol/L) & $1.2 \pm 0.4$ & $1.2 \pm 0.3$ & $1.1 \pm 0.3$ \\
\hline Fasting LDL cholesterol (mmol/L) & $3.3 \pm 0.9$ & $3.3 \pm 0.8$ & $3.3 \pm 0.8$ \\
\hline eGFR $\left(\mathrm{ml} / \mathrm{min} / 1.73 \mathrm{~cm}^{2}\right)$ & 93 [84-105] & $93[82-104]$ & 96 [84-110] \\
\hline Physically active (\%) & 56 & 64 & 68 \\
\hline Current smokers/former smokers (\%) & $16 / 55$ & $19 / 50$ & $25 / 48$ \\
\hline Pack years of smoking in ex/current smokers & $23[11-34]$ & $18[9-36]$ & $27[15-44]$ \\
\hline Use of anti-hypertensive medication (\%) & 38 & 37 & 40 \\
\hline Use of glucose-lowering medication (\%) & 15 & 10 & 15 \\
\hline Use of lipid-modifying medication (\%) & 22 & 19 & 16 \\
\hline Use of anti-thrombotic medication (\%) & 19 & 24 & 20 \\
\hline $\mathrm{CRP}(\mathrm{mg} / \mathrm{L})$ & $2.10[0.94-3.95]$ & $1.81[0.90-3.67]$ & 2.15 [1.07-4.30] \\
\hline Interleukin-6 (ng/L) & $1.68[1.15-2.36]$ & $1.43[1.00-2.20]$ & $1.60[1.18-2.20]$ \\
\hline Interleukin-8 (ng/L) & $4.46[3.55-5.81]$ & $4.26[3.49-5.20]$ & $4.49[3.82-5.74]$ \\
\hline Tumour necrosis factor- $\alpha$ (ng/L) & $6.08[5.22-7.73]$ & $6.28[5.42-7.47]$ & $6.18[5.16-7.51]$ \\
\hline Soluble intercellular adhesion molecule-1 ( $\mu \mathrm{g} / \mathrm{L}$ ) & 209 [185-249] & 213 [188-239] & $218[190-246]$ \\
\hline Serum amyloid $A$ (mg/L) & $1.36[1.02-2.35]$ & $1.33[0.95-2.27]$ & $1.50[1.02-2.23]$ \\
\hline Von Willebrand factor (\%) & 115 [84-161] & 114 [88-152] & 132 [97-182] \\
\hline Soluble E-selectin ( $\mu \mathrm{g} / \mathrm{L})$ & 8.26 [6.59-10.55] & 8.70 [6.01-10.93] & 7.67 [5.43-9.79] \\
\hline Soluble vascular cell adhesion molecule-1 ( $\mu \mathrm{g} / \mathrm{L}$ ) & 329 [294-367] & $334[295-384]$ & $332[288-382]$ \\
\hline Inflammation score & $-0.07 \pm 1.0$ & $-0.19 \pm 1.0$ & $-0.02 \pm 0.9$ \\
\hline Endothelial dysfunction score & $-0.08 \pm 1.0$ & $-0.09 \pm 0.9$ & $-0.03 \pm 1.0$ \\
\hline $\mathrm{cIMT}(\mu \mathrm{m})$ & $0.78 \pm 0.16$ & $0.79 \pm 0.17$ & $0.75 \pm 0.14$ \\
\hline Prevalent CVD (\%) & 29 & 27 & 28 \\
\hline Prevalent CVE (\%) & 15 & 14 & 18 \\
\hline
\end{tabular}

Data are presented as mean \pm standard deviation, median [interquartile range] or proportion. * data before multiple imputation were available in N=533 to 567 participants; eGFR, estimated glomerular filtration rate 


\section{b) According to tertiles of plasma MASP-1 concentrations}

\begin{tabular}{|c|c|c|c|}
\hline \multirow[t]{2}{*}{ Baseline characteristics $^{\dagger}$} & Tertile 1 & Tertile 2 & Tertile 3 \\
\hline & $n=187$ & $\mathrm{n}=188$ & $n=188$ \\
\hline $\mathrm{MBL}(\mu \mathrm{g} / \mathrm{L})$ & 969 [560-1605] & 524 [131-970] & $355[88-752]$ \\
\hline MBL genotypes, A/0 and 0/0 combined (\%) & 17 & 44 & 53 \\
\hline MASP-1 ( $\mu \mathrm{g} / \mathrm{L})$ & $5878 \pm 1183$ & $8953 \pm 748$ & $12572 \pm 2152$ \\
\hline MASP-2 ( $\mu \mathrm{g} / \mathrm{L})$ & $1049 \pm 554$ & $1230 \pm 563$ & $1414 \pm 652$ \\
\hline MASP-3 ( $\mu \mathrm{g} / \mathrm{L})$ & $7046 \pm 2001$ & $7039 \pm 2293$ & $7406 \pm 2674$ \\
\hline MAp44 ( $\mu \mathrm{g} / \mathrm{L})$ & $2360 \pm 484$ & $2397 \pm 528$ & $2566 \pm 639$ \\
\hline C3a $(\mu \mathrm{g} / \mathrm{L})$ & $60[51-74]$ & $59[48-73]$ & $58[49-70]$ \\
\hline $\mathrm{C} 5 \mathrm{a}(\mu \mathrm{g} / \mathrm{L})$ & $7.7 \pm 3.6$ & $7.5 \pm 4.1$ & $7.6 \pm 3.7$ \\
\hline $\mathrm{sC} 5 \mathrm{~b}-9(\mu \mathrm{g} / \mathrm{L})$ & $113 \pm 32$ & $112 \pm 37$ & $115 \pm 36$ \\
\hline Age (years) & $60 \pm 7$ & $59 \pm 7$ & $59 \pm 7$ \\
\hline Sex $(\%$ men $)$ & 66 & 64 & 54 \\
\hline Normal/impaired glucose metabolism/T2DM (\%) & $51 / 23 / 26$ & $55 / 21 / 24$ & $50 / 24 / 26$ \\
\hline $\mathrm{HbA} 1 \mathrm{c}(\%)$ & $5.9 \pm 0.8$ & $6.0 \pm 0.8$ & $6.0 \pm 0.9$ \\
\hline HOMA2-IR & $1.77[1.19-2.96]$ & $1.61[1.11-2.40]$ & $1.47[1.01-2.40]$ \\
\hline Waist $(\mathrm{cm})$ & $101 \pm 11$ & $100 \pm 12$ & $97 \pm 12$ \\
\hline Systolic blood pressure (mmHg) & $142 \pm 20$ & $138 \pm 18$ & $140 \pm 20$ \\
\hline Diastolic blood pressure (mmHg) & $83 \pm 9$ & $81 \pm 9$ & $82 \pm 10$ \\
\hline Fasting triacylglycerols (mmol/L) & $1.40[1.00-1.80]$ & $1.40[1.00-1.90]$ & $1.50[1.00-2.00]$ \\
\hline Fasting cholesterol (mmol/L) & $5.1 \pm 0.9$ & $5.2 \pm 0.9$ & $5.4 \pm 1.1$ \\
\hline Fasting HDL cholesterol (mmol/L) & $1.15 \pm 0.34$ & $1.18 \pm 0.34$ & $1.24 \pm 0.37$ \\
\hline Fasting LDL cholesterol (mmol/L) & $3.2 \pm 0.8$ & $3.3 \pm 0.9$ & $3.3 \pm 0.9$ \\
\hline $\mathrm{eGFR}\left(\mathrm{ml} / \mathrm{min} / 1.73 \mathrm{~cm}^{2}\right)$ & 94 [83-109] & $92[83-103]$ & $96[85-106]$ \\
\hline Physically active (\%) & 67 & 55 & 66 \\
\hline Current smokers/former smokers (\%) & $22 / 48$ & $19 / 54$ & $19 / 51$ \\
\hline Pack years of smoking in ex/current smokers & 26 [15-43] & $20[11-34]$ & $23[11-36]$ \\
\hline Use of anti-hypertensive medication (\%) & 40 & 37 & 38 \\
\hline Use of glucose-lowering medication (\%) & 14 & 11 & 15 \\
\hline Use of lipid-modifying medication (\%) & 18 & 15 & 25 \\
\hline Use of anti-thrombotic medication (\%) & 21 & 20 & 22 \\
\hline $\mathrm{CRP}(\mathrm{mg} / \mathrm{L})$ & $2.24[1.08-5.01]$ & $2.09[0.84-3.75]$ & $1.87[0.97-3.35]$ \\
\hline Interleukin-6 (ng/L) & $1.59[1.23-2.32]$ & $1.58[1.11-2.47]$ & $1.46[1.08-2.18]$ \\
\hline Interleukin-8 (ng/L) & $4.37[3.60-5.58]$ & $4.30[3.59-5.47]$ & $4.58[3.64-5.68]$ \\
\hline Tumour necrosis factor- $\alpha$ (ng/L) & $6.42[5.51-7.75]$ & $6.12[5.14-7.81]$ & $6.15[5.26-7.35]$ \\
\hline Soluble intercellular adhesion molecule-1 ( $\mu \mathrm{g} / \mathrm{L})$ & 213 [192-245] & 217 [188-245] & 209 [184-242] \\
\hline Serum amyloid $A$ (mg/L) & $1.54[1.06-2.45]$ & $1.49[1.00-2.35]$ & $1.28[0.89-1.94]$ \\
\hline Von Willebrand factor (\%) & 127 [97-165] & 117 [89-156] & $114[84-162]$ \\
\hline Soluble E-selectin ( $\mu \mathrm{g} / \mathrm{L})$ & $8.31[6.55-10.2]$ & $8.01[5.91-10.5]$ & $7.98[5.74-10.4]$ \\
\hline Soluble vascular cell adhesion molecule-1 ( $\mu \mathrm{g} / \mathrm{L}$ ) & 336 [296-386] & 329 [292-372] & $331[289-372]$ \\
\hline Inflammation score & $0.03 \pm 0.94$ & $-0.08 \pm 1.03$ & $-0.21 \pm 0.84$ \\
\hline Endothelial dysfunction score & $0.03 \pm 0.93$ & $-0.04 \pm 0.98$ & $-0.18 \pm 0.96$ \\
\hline $\operatorname{cIMT}(\mu \mathrm{m})$ & $777 \pm 158$ & $773 \pm 158$ & $772 \pm 165$ \\
\hline Prevalent CVD (\%) & 29 & 23 & 30 \\
\hline Prevalent CVE (\%) & 19 & 13 & 14 \\
\hline
\end{tabular}

Data are presented as mean \pm standard deviation, median [interquartile range] or proportion.

${ }^{+}$data before multiple imputation were available in $\mathrm{N}=533$ to 567 participants

eGFR, estimated glomerular filtration rate 


\section{c) According to tertiles of plasma MASP-2 concentrations}

\begin{tabular}{|c|c|c|c|}
\hline \multirow[t]{2}{*}{ Baseline characteristics ${ }^{\ddagger}$} & Tertile 1 & Tertile 2 & Tertile 3 \\
\hline & $\mathrm{n}=188$ & $n=189$ & $\mathrm{n}=188$ \\
\hline $\mathrm{MBL}(\mu \mathrm{g} / \mathrm{L})$ & $902[524-1354]$ & 553 [164-994] & $314[109-804]$ \\
\hline MBL genotypes, $\mathrm{A} / 0$ and $0 / 0$ combined (\%) & 20 & 42 & 52 \\
\hline MASP-1 ( $\mu \mathrm{g} / \mathrm{L})$ & $8088 \pm 3062$ & $9456 \pm 2884$ & $9870 \pm 3133$ \\
\hline MASP-2 ( $\mu \mathrm{g} / \mathrm{L})$ & $661 \pm 218$ & $1126 \pm 117$ & $1904 \pm 510$ \\
\hline MASP-3 ( $\mu \mathrm{g} / \mathrm{L})$ & $6965 \pm 2392$ & $7051 \pm 2057$ & $7429 \pm 2522$ \\
\hline MAp44 ( $\mu \mathrm{g} / \mathrm{L})$ & $2284 \pm 447$ & $2480 \pm 593$ & $2551 \pm 601$ \\
\hline $\mathrm{C} 3 \mathrm{a}(\mu \mathrm{g} / \mathrm{L})$ & $58[49-72]$ & $58[48-71]$ & $61[51-76]$ \\
\hline $\mathrm{C} 5 \mathrm{a}(\mu \mathrm{g} / \mathrm{L})$ & $7.0 \pm 3.6$ & $7.7 \pm 3.7$ & $8.1 \pm 4.1$ \\
\hline $\mathrm{sC} 5 \mathrm{~b}-9(\mu \mathrm{g} / \mathrm{L})$ & $110 \pm 36$ & $111 \pm 36$ & $118 \pm 33$ \\
\hline Age (years) & $60 \pm 7$ & $59 \pm 7$ & $60 \pm 7$ \\
\hline Sex $(\%$ men $)$ & 65 & 61 & 58 \\
\hline Normal/impaired glucose metabolism/T2DM (\%) & $57 / 21 / 22$ & $53 / 22 / 25$ & $47 / 24 / 29$ \\
\hline $\mathrm{HbA} 1 \mathrm{c}(\%)$ & $6.0 \pm 0.9$ & $6.0 \pm 0.8$ & $6.0 \pm 0.7$ \\
\hline HOMA2-IR & $1.59[1.12-2.34]$ & $1.47[1.07-2.65]$ & $1.86[1.14-2.74]$ \\
\hline Waist $(\mathrm{cm})$ & $98 \pm 12$ & $99 \pm 11$ & $100 \pm 12$ \\
\hline Systolic blood pressure (mmHg) & $140 \pm 22$ & $139 \pm 18$ & $141 \pm 18$ \\
\hline Diastolic blood pressure $(\mathrm{mmHg})$ & $82 \pm 10$ & $82 \pm 9$ & $82 \pm 9$ \\
\hline Fasting triacylglycerols (mmol/L) & $1.30[0.90-1.78]$ & $1.40[1.00-2.00]$ & $1.50[1.10-2.00]$ \\
\hline Fasting cholesterol (mmol/L) & $5.0 \pm 0.9$ & $5.2 \pm 0.9$ & $5.4 \pm 1.1$ \\
\hline Fasting HDL cholesterol (mmol/L) & $1.2 \pm 0.3$ & $1.2 \pm 0.3$ & $1.2 \pm 0.4$ \\
\hline Fasting LDL cholesterol (mmol/L) & $3.2 \pm 0.9$ & $3.3 \pm 0.8$ & $3.4 \pm 0.9$ \\
\hline $\mathrm{eGFR}\left(\mathrm{ml} / \mathrm{min} / 1.73 \mathrm{~cm}^{2}\right)$ & $94[82-105]$ & 97 [85-108] & 92 [83-104] \\
\hline Physically active (\%) & 64 & 65 & 60 \\
\hline Current smokers/former smokers (\%) & $18 / 46$ & $26 / 48$ & $17 / 59$ \\
\hline Pack years of smoking in ex/current smokers & $24[13-38]$ & $25[12-37]$ & $21[11-34]$ \\
\hline Use of anti-hypertensive medication (\%) & 38 & 35 & 42 \\
\hline Use of glucose-lowering medication (\%) & 12 & 15 & 14 \\
\hline Use of lipid-modifying medication (\%) & 12 & 20 & 26 \\
\hline Use of anti-thrombotic medication (\%) & 19 & 22 & 22 \\
\hline $\mathrm{CRP}(\mathrm{mg} / \mathrm{L})$ & $1.91[0.91-3.49]$ & $1.87[0.98-3.75]$ & $2.28[0.99-4.47]$ \\
\hline Interleukin-6 (ng/L) & $1.51[1.12-2.19]$ & $1.59[1.11-2.18]$ & $1.63[1.15-2.37]$ \\
\hline Interleukin-8 (ng/L) & $4.32[3.60-5.53]$ & $4.41[3.55-5.84]$ & $4.46[3.62-5.48]$ \\
\hline Tumour necrosis factor- $\alpha$ (ng/L) & $6.34[5.23-7.66]$ & $6.08[5.11-7.39]$ & $6.28[5.41-7.69]$ \\
\hline Soluble intercellular adhesion molecule-1 ( $\mu \mathrm{g} / \mathrm{L})$ & $212[187-240]$ & 210 [189-244] & $217[188-251]$ \\
\hline Serum amyloid $A(\mathrm{mg} / \mathrm{L})$ & $1.31[0.95-2.24]$ & $1.47[1.06-2.35]$ & $1.46[0.95-2.26]$ \\
\hline Von Willebrand factor (\%) & 124 [97-165] & $116[86-152]$ & $117[83-164]$ \\
\hline Soluble E-selectin $(\mu \mathrm{g} / \mathrm{L})$ & $8.08[5.97-10.1]$ & $8.49[5.89-10.5]$ & $8.06[6.13-10.7]$ \\
\hline Soluble vascular cell adhesion molecule-1 ( $\mu \mathrm{g} / \mathrm{L})$ & 335 [294-394] & 327 [293-376] & $332[293-373]$ \\
\hline Inflammation score & $-0.15 \pm 1.00$ & $-0.09 \pm 0.89$ & $-0.03 \pm 0.94$ \\
\hline Endothelial dysfunction score & $-0.04 \pm 0.88$ & $-0.12 \pm 0.96$ & $-0.03 \pm 1.04$ \\
\hline $\mathrm{cIMT}(\mu \mathrm{m})$ & $0.77 \pm 0.16$ & $0.78 \pm 0.15$ & $0.77 \pm 0.17$ \\
\hline Prevalent CVD (\%) & 26 & 28 & 29 \\
\hline Prevalent CVE (\%) & 16 & 15 & 16 \\
\hline
\end{tabular}

Data are presented as mean \pm standard deviation, median [interquartile range] or proportion.

₹ data before multiple imputation were available in $N=533$ to 567 participants

eGFR, estimated glomerular filtration rate 


\section{d) According to tertiles of plasma MASP-3 concentrations}

\begin{tabular}{|c|c|c|c|}
\hline \multirow[t]{2}{*}{ Baseline characteristics $\S$} & Tertile 1 & Tertile 2 & Tertile 3 \\
\hline & $\mathrm{n}=187$ & $\mathrm{n}=188$ & $\mathrm{n}=188$ \\
\hline $\mathrm{MBL}(\mu \mathrm{g} / \mathrm{L})$ & 565 [183-1039] & 633 [189-1137] & $640[187-1184]$ \\
\hline MBL genotypes, $\mathrm{A} / 0$ and $0 / 0$ combined (\%) & 40 & 38 & 37 \\
\hline MASP-1 $(\mu \mathrm{g} / \mathrm{L})$ & $9276 \pm 2987$ & $8688 \pm 3066$ & $9477 \pm 3232$ \\
\hline MASP-2 ( $\mu \mathrm{g} / \mathrm{L})$ & $1186 \pm 621$ & $1234 \pm 621$ & $1265 \pm 582$ \\
\hline MASP-3 ( $\mu \mathrm{g} / \mathrm{L})$ & $4987 \pm 716$ & $6810 \pm 494$ & $9673 \pm 2115$ \\
\hline MAp44 ( $\mu \mathrm{g} / \mathrm{L})$ & $2317 \pm 484$ & $2375 \pm 474$ & $2627 \pm 663$ \\
\hline C3a $(\mu \mathrm{g} / \mathrm{L})$ & $60[59-71]$ & $58[48-73]$ & $59[51-75]$ \\
\hline $\mathrm{C} 5 \mathrm{a}(\mu \mathrm{g} / \mathrm{L})$ & $7.1 \pm 3.6$ & $7.7 \pm 3.9$ & $8.0 \pm 4.0$ \\
\hline $\mathrm{sC} 5 \mathrm{~b}-9(\mu \mathrm{g} / \mathrm{L})$ & $111 \pm 33$ & $115 \pm 36$ & $115 \pm 36$ \\
\hline Age (years) & $60 \pm 7$ & $60 \pm 7$ & $59 \pm 6$ \\
\hline Sex $(\%$ men $)$ & 63 & 61 & 59 \\
\hline Normal/impaired glucose metabolism/T2DM (\%) & $58 / 23 / 19$ & $52 / 23 / 25$ & $46 / 21 / 33$ \\
\hline $\mathrm{HbA} 1 \mathrm{c}(\%)$ & $5.9 \pm 0.8$ & $6.0 \pm 0.8$ & $6.1 \pm 0.9$ \\
\hline HOMA2-IR & $1.38[0.99-2.22]$ & $1.48[1.04-2.39]$ & $2.06[1.35-3.49]$ \\
\hline Waist $(\mathrm{cm})$ & $98 \pm 11$ & $98 \pm 11$ & $102 \pm 13$ \\
\hline Systolic blood pressure (mmHg) & $140 \pm 20$ & $141 \pm 19$ & $140 \pm 19$ \\
\hline Diastolic blood pressure (mmHg) & $82 \pm 9$ & $82 \pm 9$ & $83 \pm 10$ \\
\hline Fasting triacylglycerols (mmol/L) & $1.30[0.90-1.80]$ & $1.40[0.90-1.90]$ & $1.60[1.10-2.08]$ \\
\hline Fasting cholesterol (mmol/L) & $5.2 \pm 1.0$ & $5.2 \pm 0.9$ & $5.3 \pm 1.0$ \\
\hline Fasting HDL cholesterol (mmol/L) & $1.2 \pm 0.4$ & $1.2 \pm 0.4$ & $1.1 \pm 0.3$ \\
\hline Fasting LDL cholesterol (mmol/L) & $3.3 \pm 0.9$ & $3.3 \pm 0.8$ & $3.4 \pm 0.9$ \\
\hline $\mathrm{eGFR}\left(\mathrm{ml} / \mathrm{min} / 1.73 \mathrm{~cm}^{2}\right)$ & $94[83-104]$ & $93[81-104]$ & $96[85-111]$ \\
\hline Physically active (\%) & 65 & 64 & 59 \\
\hline Current smokers/former smokers (\%) & $21 / 49$ & $20 / 48$ & $19 / 55$ \\
\hline Pack years of smoking in ex/current smokers & $21[12-38]$ & $24[13-34]$ & $24[13-34]$ \\
\hline Use of anti-hypertensive medication (\%) & 36 & 40 & 38 \\
\hline Use of glucose-lowering medication (\%) & 10 & 16 & 14 \\
\hline Use of lipid-modifying medication (\%) & 19 & 22 & 16 \\
\hline Use of anti-thrombotic medication (\%) & 20 & 23 & 19 \\
\hline $\operatorname{CRP}(\mathrm{mg} / \mathrm{L})$ & $1.85[0.98-3.75]$ & $1.84[0.86-3.86]$ & $2.34[1.11-4.30]$ \\
\hline Interleukin-6 (ng/L) & $1.52[1.11-2.21]$ & $1.55[1.12-2.25]$ & $1.58[1.21-2.31]$ \\
\hline Interleukin-8 (ng/L) & $4.24[3.50-5.41]$ & $4.46[3.70-5.60]$ & $4.56[3.70-5.76]$ \\
\hline Tumour necrosis factor- $\alpha$ (ng/L) & $6.11[5.27-7.50]$ & $6.30[5.21-7.62]$ & $6.26[5.27-7.72]$ \\
\hline Soluble intercellular adhesion molecule-1 ( $\mu \mathrm{g} / \mathrm{L}$ ) & 208 [184-239] & 209 [186-238] & 223 [198-254] \\
\hline Serum amyloid $A$ (mg/L) & $1.33[0.98-2.32]$ & $1.49[1.00-2.27]$ & $1.46[1.01-2.26]$ \\
\hline Von Willebrand factor (\%) & $114[77-156]$ & $121[92-164]$ & $121[94-163]$ \\
\hline Soluble E-selectin ( $\mu \mathrm{g} / \mathrm{L})$ & $7.96[5.75-9.76]$ & $8.00[5.97-10.3]$ & $8.74[6.50-11.9]$ \\
\hline Soluble vascular cell adhesion molecule-1 ( $\mu \mathrm{g} / \mathrm{L})$ & $329[290-382]$ & 329 [293-365] & 337 [296-386] \\
\hline Inflammation score & $-0.17 \pm 0.93$ & $-0.12 \pm 0.99$ & $0.04 \pm 0.90$ \\
\hline Endothelial dysfunction score & $-0.22 \pm 0.88$ & $-0.11 \pm 0.95$ & $0.16 \pm 1.01$ \\
\hline $\mathrm{cIMT}(\mu \mathrm{m})$ & $0.77 \pm 0.15$ & $0.77 \pm 0.16$ & $0.78 \pm 0.16$ \\
\hline Prevalent CVD (\%) & 24 & 33 & 25 \\
\hline Prevalent CVE (\%) & 16 & 18 & 12 \\
\hline
\end{tabular}

Data are presented as mean \pm standard deviation, median [interquartile range] or proportion.

$\S$ data before multiple imputation were available in $\mathrm{N}=533$ to 567 participants eGFR, estimated glomerular filtration rate 


\section{e) According to tertiles of plasma MAp44 concentrations}

\begin{tabular}{|c|c|c|c|}
\hline \multirow[t]{2}{*}{ Baseline characteristics $\|$} & Tertile 1 & Tertile 2 & Tertile 3 \\
\hline & $\mathrm{n}=189$ & $n=189$ & $n=189$ \\
\hline $\mathrm{MBL}(\mu \mathrm{g} / \mathrm{L})$ & 522 [146-929] & $805[234-1218]$ & 609 [169-1166] \\
\hline MBL genotypes, $\mathrm{A} / 0$ and $0 / 0$ combined (\%) & 43 & 33 & 38 \\
\hline MASP-1 ( $\mu \mathrm{g} / \mathrm{L})$ & $8596 \pm 2836$ & $8879 \pm 3043$ & $9960 \pm 3292$ \\
\hline MASP-2 ( $\mu \mathrm{g} / \mathrm{L})$ & $1081 \pm 534$ & $1250 \pm 609$ & $1363 \pm 647$ \\
\hline MASP-3 ( $\mu \mathrm{g} / \mathrm{L})$ & $6439 \pm 1929$ & $7278 \pm 2160$ & $7756 \pm 2682$ \\
\hline MAp44 ( $\mu \mathrm{g} / \mathrm{L})$ & $1962 \pm 186$ & $2365 \pm 130$ & $3041 \pm 505$ \\
\hline $\mathrm{C} 3 \mathrm{a}(\mu \mathrm{g} / \mathrm{L})$ & $60[59-71]$ & 58 [48-73] & $59[51-75]$ \\
\hline $\mathrm{C} 5 \mathrm{a}(\mu \mathrm{g} / \mathrm{L})$ & $7.1 \pm 3.6$ & $7.7 \pm 3.9$ & $8.0 \pm 4.0$ \\
\hline sC5b-9 ( $\mu \mathrm{g} / \mathrm{L})$ & $111 \pm 33$ & $115 \pm 36$ & $115 \pm 36$ \\
\hline Age (years) & $60 \pm 7$ & $60 \pm 6$ & $59 \pm 7$ \\
\hline Sex $(\%$ men $)$ & 60 & 62 & 61 \\
\hline Normal/impaired glucose metabolism/T2DM (\%) & $62 / 20 / 18$ & $48 / 23 / 29$ & $47 / 24 / 29$ \\
\hline $\mathrm{HbA} 1 \mathrm{c}(\%)$ & $5.9 \pm 0.9$ & $6.0 \pm 0.7$ & $6.0 \pm 0.8$ \\
\hline HOMA2-IR & $1.23[0.89-2.01]$ & $1.65[1.13-2.45]$ & $2.02[1.43-3.29]$ \\
\hline Waist $(\mathrm{cm})$ & $96 \pm 11$ & $100 \pm 11$ & $102 \pm 12$ \\
\hline Systolic blood pressure (mmHg) & $138 \pm 20$ & $141 \pm 20$ & $142 \pm 18$ \\
\hline Diastolic blood pressure (mmHg) & $80 \pm 9$ & $82 \pm 10$ & $83 \pm 9$ \\
\hline Fasting triacylglycerols (mmol/L) & $1.10[0.80-1.40]$ & $1.50[1.10-2.00]$ & $1.70[1.30-2.20]$ \\
\hline Fasting cholesterol (mmol/L) & $5.0 \pm 0.9$ & $5.2 \pm 0.9$ & $5.4 \pm 1.1$ \\
\hline Fasting HDL cholesterol (mmol/L) & $1.3 \pm 0.4$ & $1.2 \pm 0.3$ & $1.1 \pm 0.3$ \\
\hline Fasting LDL cholesterol (mmol/L) & $3.1 \pm 0.8$ & $3.4 \pm 0.8$ & $3.4 \pm 0.9$ \\
\hline eGFR $\left(\mathrm{ml} / \mathrm{min} / 1.73 \mathrm{~cm}^{2}\right)$ & $95[85-104]$ & 92 [79-104] & $95[84-111]$ \\
\hline Physically active (\%) & 60 & 67 & 61 \\
\hline Current smokers/former smokers (\%) & $22 / 48$ & $23 / 51$ & $16 / 53$ \\
\hline Pack years of smoking in ex/current smokers & $23[12-41]$ & $24[12-35]$ & $21[12-36]$ \\
\hline Use of anti-hypertensive medication (\%) & 36 & 40 & 39 \\
\hline Use of glucose-lowering medication (\%) & 13 & 16 & 11 \\
\hline Use of lipid-modifying medication (\%) & 20 & 18 & 20 \\
\hline Use of anti-thrombotic medication (\%) & 21 & 22 & 20 \\
\hline $\mathrm{CRP}(\mathrm{mg} / \mathrm{L})$ & $1.81[0.86-3.87]$ & $2.07[0.97-3.85]$ & 2.28 [1.09-4.50] \\
\hline Interleukin-6 (ng/L) & $1.57[1.12-2.42]$ & $1.54[1.16-2.17]$ & 1.58 [1.09-2.23] \\
\hline Interleukin-8 (ng/L) & $4.17[3.425 .31]$ & $4.54[3.71-5.51]$ & $4.54[3.70-5.84]$ \\
\hline Tumour necrosis factor- $\alpha$ (ng/L) & $6.18[5.26-7.63]$ & $6.03[5.13-7.31]$ & $6.48[5.44-7.85]$ \\
\hline Soluble intercellular adhesion molecule-1 ( $\mu \mathrm{g} / \mathrm{L}$ ) & 203 [182-237] & 213 [187-243] & $227[198-253]$ \\
\hline Serum amyloid $A$ (mg/L) & $1.27[0.92-2.21]$ & $1.54[1.08-2.25]$ & $1.40[0.98-2.34]$ \\
\hline Von Willebrand factor (\%) & $109[81-151]$ & $130[97-172]$ & $120[92-163]$ \\
\hline Soluble E-selectin ( $\mu \mathrm{g} / \mathrm{L})$ & $7.94[5.80-9.75]$ & $7.84[5.88-10.3]$ & $8.90[6.71-11.5]$ \\
\hline Soluble vascular cell adhesion molecule-1 ( $\mu \mathrm{g} / \mathrm{L}$ ) & 327 [289-377] & $331[293-374]$ & $333[296-386]$ \\
\hline Inflammation score & $-0.21 \pm 0.94$ & $-0.10 \pm 0.90$ & $0.03 \pm 0.98$ \\
\hline Endothelial dysfunction score & $-0.30 \pm 0.86$ & $-0.02 \pm 0.93$ & $0.13 \pm 1.03$ \\
\hline $\operatorname{cIMT}(\mu \mathrm{m})$ & $0.75 \pm 0.15$ & $0.78 \pm 0.16$ & $0.79 \pm 0.16$ \\
\hline Prevalent CVD (\%) & 27 & 27 & 30 \\
\hline Prevalent CVE (\%) & 15 & 16 & 15 \\
\hline
\end{tabular}

Data are presented as mean \pm standard deviation, median [interquartile range] or proportion.

II data before multiple imputation were available in $\mathrm{N}=533$ to 567 participants eGFR, estimated glomerular filtration rate 


\begin{tabular}{|c|c|c|c|}
\hline \multicolumn{4}{|l|}{ f) According to MBL genotypes } \\
\hline \multirow[t]{2}{*}{ Baseline characteristics \# } & $A / A$ & $\mathrm{~A} / 0$ & $0 / 0$ \\
\hline & $n=343$ & $n=181$ & $n=31$ \\
\hline $\mathrm{MBL}(\mu \mathrm{g} / \mathrm{L})$ & 923 [497-1902] & $183[33-326]$ & $0.1[0.1-0.1]$ \\
\hline MBL genotypes, $\mathrm{A} / 0$ and $0 / 0$ combined $(\%)$ & - & - & - \\
\hline MASP-1 ( $\mu \mathrm{g} / \mathrm{L})$ & $8368 \pm 2998$ & $10394 \pm 2953$ & $10154 \pm 2426$ \\
\hline MASP-2 ( $\mu \mathrm{g} / \mathrm{L})$ & $1101 \pm 529$ & $1435 \pm 656$ & $1505 \pm 719$ \\
\hline MASP-3 ( $\mu \mathrm{g} / \mathrm{L})$ & $7143 \pm 2297$ & $7190 \pm 2327$ & $7128 \pm 2606$ \\
\hline MAp44 ( $\mu \mathrm{g} / \mathrm{L})$ & $2453 \pm 500$ & $2429 \pm 683$ & $2392 \pm 480$ \\
\hline C3a $(\mu \mathrm{g} / \mathrm{L})$ & $60[50-74]$ & 59 [49-72] & 55 [42-69] \\
\hline $\mathrm{C5a}(\mu \mathrm{g} / \mathrm{L})$ & $7.4 \pm 3.6$ & $7.9 \pm 4.2$ & $7.5 \pm 4.2$ \\
\hline $\mathrm{sC} 5 \mathrm{~b}-9(\mu \mathrm{g} / \mathrm{L})$ & $115 \pm 36$ & $112 \pm 34$ & $109 \pm 27$ \\
\hline Age (years) & $60 \pm 7$ & $59 \pm 7$ & $59 \pm 7$ \\
\hline Sex (\%men) & 62 & 60 & 71 \\
\hline Normal/impaired glucose metabolism/T2DM (\%) & $53 / 22 / 25$ & $50 / 22 / 28$ & $68 / 16 / 16$ \\
\hline $\mathrm{HbA} 1 \mathrm{c}(\%)$ & $6.0 \pm 0.8$ & $6.0 \pm 0.9$ & $5.8 \pm 0.5$ \\
\hline HOMA2-IR & $1.65[1.13-2.62]$ & $1.51[1.03-2.42]$ & $1.88[1.10-2.65]$ \\
\hline Waist $(\mathrm{cm})$ & $99 \pm 12$ & $100 \pm 12$ & $99 \pm 10$ \\
\hline Systolic blood pressure (mmHg) & $141 \pm 20$ & $140 \pm 19$ & $138 \pm 17$ \\
\hline Diastolic blood pressure ( $\mathrm{mmHg}$ ) & $81 \pm 9$ & $82 \pm 9$ & $79 \pm 9$ \\
\hline Fasting triacylglycerols (mmol/L) & $1.40[1.00-2.00]$ & $1.40[0.90-1.90]$ & $1.40[1.00-2.00]$ \\
\hline Fasting cholesterol (mmol/L) & $5.2 \pm 0.9$ & $5.2 \pm 1.11$ & $5.2 \pm 1.01$ \\
\hline Fasting HDL cholesterol (mmol/L) & $1.2 \pm 0.4$ & $1.2 \pm 0.3$ & $1.2 \pm 0.3$ \\
\hline Fasting LDL cholesterol (mmol/L) & $3.3 \pm 0.9$ & $3.3 \pm 0.9$ & $3.2 \pm 0.9$ \\
\hline eGFR $\left(\mathrm{ml} / \mathrm{min} / 1.73 \mathrm{~cm}^{2}\right)$ & $94[83-108]$ & 95 [84-105] & 88 [82-97] \\
\hline Physically active (\%) & 65 & 57 & 63 \\
\hline Current smokers/former smokers (\%) & $23 / 48$ & $17 / 53$ & 13/61 \\
\hline Pack years of smoking in ex/current smokers & 24 [14-39] & $24[11-35]$ & 14 [9-23] \\
\hline Use of anti-hypertensive medication (\%) & 37 & 39 & 45 \\
\hline Use of glucose-lowering medication (\%) & 12 & 16 & 7 \\
\hline Use of lipid-modifying medication (\%) & 18 & 20 & 19 \\
\hline Use of anti-thrombotic medication (\%) & 21 & 21 & 23 \\
\hline $\mathrm{CRP}(\mathrm{mg} / \mathrm{L})$ & $1.99[0.97-3.86]$ & $2.14[0.95-4.03]$ & $2.10[1.00-3.07]$ \\
\hline Interleukin-6 (ng/L) & $1.55[1.14-2.21]$ & $1.62[1.10-2.31]$ & $1.93[1.19-2.62]$ \\
\hline Interleukin-8 (ng/L) & $4.42[3.70-5.56]$ & $4.31[3.51-5.47]$ & $4.45[3.19-6.21]$ \\
\hline Tumour necrosis factor- $\alpha$ (ng/L) & $6.14[5.28-7.34]$ & $6.25[5.19-7.92]$ & $6.73[5.54-7.42]$ \\
\hline Soluble intercellular adhesion molecule-1 ( $\mu \mathrm{g} / \mathrm{L})$ & 215 [188-244] & 213 [188-249] & $200[186-251]$ \\
\hline Serum amyloid $A$ (mg/L) & $1.44[0.98-2.25]$ & $1.43[1.02-2.35]$ & $1.36[0.89-2.17]$ \\
\hline Von Willebrand factor (\%) & $121[88-163]$ & 115 [92-155] & $110[89-176]$ \\
\hline Soluble E-selectin ( $\mu \mathrm{g} / \mathrm{L})$ & $8.28[6.01-10.5]$ & $8.11[5.94-10.08]$ & $8.60[5.95-11.29]$ \\
\hline Soluble vascular cell adhesion molecule-1 ( $\mu \mathrm{g} / \mathrm{L}$ ) & 329 [289-381] & 339 [297-378] & 324 [292-386] \\
\hline Inflammation score & $-0.10 \pm 0.92$ & $-0.05 \pm 1.00$ & $-0.08 \pm 0.89$ \\
\hline Endothelial dysfunction score & $-0.07 \pm 0.98$ & $-0.04 \pm 0.96$ & $-0.05 \pm 0.77$ \\
\hline $\operatorname{cIMT}(\mu \mathrm{m})$ & $0.77 \pm 0.15$ & $0.78 \pm 0.17$ & $0.75 \pm 0.14$ \\
\hline Prevalent CVD (\%) & 29 & 23 & 36 \\
\hline Prevalent CVE (\%) & 17 & 12 & 23 \\
\hline
\end{tabular}

Data are presented as mean \pm standard deviation, median [interquartile range] or proportion.

\# data before multiple imputation were available in $\mathrm{N}=533$ to 567 participants

eGFR, estimated glomerular filtration rate 
Supplemental table 2: Baseline characteristics of the participants free of CVD at baseline, stratified for incident CVD at follow-up

\begin{tabular}{|c|c|c|}
\hline \multirow[t]{2}{*}{ Baseline characteristics } & \multicolumn{2}{|c|}{ Participants free of CVD at baseline } \\
\hline & $\begin{array}{c}\text { no CVD } \\
\text { at follow-up } \\
N=269\end{array}$ & $\begin{array}{c}\text { CVD } \\
\text { at follow-up } \\
N=73\end{array}$ \\
\hline $\mathrm{MBL}(\mu \mathrm{g} / \mathrm{L})$ & 690 [228-1218] & $529[133-891]$ \\
\hline MBL genotypes, $\mathrm{A} / 0$ and $0 / 0$ combined (\%) & $35 \%$ & $40 \%$ \\
\hline MASP-1 ( $\mu \mathrm{g} / \mathrm{L})$ & $9304 \pm 3042$ & $8729 \pm 3210$ \\
\hline MASP-2 ( $\mu \mathrm{g} / \mathrm{L})$ & $1228 \pm 629$ & $1170 \pm 616$ \\
\hline MASP-3 ( $\mu \mathrm{g} / \mathrm{L})$ & $7040 \pm 2418$ & $7566 \pm 571$ \\
\hline MAp44 ( $\mu \mathrm{g} / \mathrm{L})$ & $2445 \pm 526$ & $2423 \pm 571$ \\
\hline Age (years) & $58 \pm 7$ & $59 \pm 7$ \\
\hline Sex (\%men) & 60 & 59 \\
\hline Normal/impaired glucose metabolism/T2DM (\%) & $59 / 24 / 17$ & $58 / 16 / 26$ \\
\hline $\mathrm{HbA} 1 \mathrm{c}(\%)$ & $5.84 \pm 0.77$ & $5.90 \pm 0.65$ \\
\hline HOMA2-IR & $1.49[1.07-2.14]$ & $1.53[1.06-2.56]$ \\
\hline Waist $(\mathrm{cm})$ & $97 \pm 12$ & $99 \pm 13$ \\
\hline Systolic blood pressure (mmHg) & $136 \pm 18$ & $140 \pm 18$ \\
\hline Diastolic blood pressure (mmHg) & $81 \pm 9$ & $83 \pm 9$ \\
\hline Fasting triacylglycerols (mmol/L) & $1.40[0.90-1.90]$ & $1.40[0.90-2.00]$ \\
\hline Fasting cholesterol (mmol/L) & $5.28 \pm 0.99$ & $5.29 \pm 0.98$ \\
\hline Fasting HDL cholesterol (mmol/L) & $1.23 \pm 0.35$ & $1.19 \pm 0.36$ \\
\hline Fasting LDL cholesterol (mmol/L) & $3.35 \pm 0.87$ & $3.41 \pm 0.92$ \\
\hline eGFR $\left(\mathrm{ml} / \mathrm{min} / 1.73 \mathrm{~cm}^{2}\right)$ & 94 [84-107] & $95[84-104]$ \\
\hline Physically active (\%) & 63 & 55 \\
\hline Current smokers/former smokers (\%) & $20 / 47$ & $14 / 51$ \\
\hline Pack years of smoking in ex/current smokers & $22[12-35]$ & 18 [10-39] \\
\hline Use of anti-hypertensive medication (\%) & 24 & 38 \\
\hline Use of glucose-lowering medication (\%) & 7 & 14 \\
\hline Use of lipid-modifying medication (\%) & 10 & 10 \\
\hline Use of anti-thrombotic medication (\%) & 5 & 18 \\
\hline $\mathrm{CRP}(\mathrm{mg} / \mathrm{L})$ & $1.56[0.84-3.21]$ & $2.55[1.02-3.41]$ \\
\hline Interleukin-6 (ng/L) & $1.34[0.99-1.95]$ & $1.61[1.12-2.22]$ \\
\hline Interleukin-8 (ng/L) & $4.32[3.47-5.47]$ & $4.19[3.50-5.20]$ \\
\hline Tumour necrosis factor- $\alpha$ (ng/L) & $6.08[5.16-7.19]$ & $5.91[5.18-7.30]$ \\
\hline Soluble intercellular adhesion molecule-1 ( $\mu \mathrm{g} / \mathrm{L}$ ) & 204 [182-237] & 210 [186-235] \\
\hline Serum amyloid $A$ (mg/L) & $1.33[0.94-1.96]$ & $1.49[1.10-2.54]$ \\
\hline Von Willebrand factor (\%) & 113 [88-155] & 126 [91-178] \\
\hline Soluble E-selectin ( $\mu \mathrm{g} / \mathrm{L})$ & $7.85[5.94-9.94]$ & $8.18[5.68-10.10]$ \\
\hline Soluble vascular cell adhesion molecule-1 ( $\mu \mathrm{g} / \mathrm{L})$ & $324[285-363]$ & 322 [293-369] \\
\hline Inflammation score & $-0.34 \pm 0.85$ & $-0.10 \pm 0.92$ \\
\hline Endothelial dysfunction score & $-0.26 \pm 0.91$ & $-0.09 \pm 0.88$ \\
\hline $\operatorname{clMT}(\mu \mathrm{m})$ & $753 \pm 155$ & $793 \pm 169$ \\
\hline
\end{tabular}

Data are presented as mean \pm standard deviation, median [interquartile range] or proportion. eGFR, estimated glomerular filtration rate 


\section{Chapter 9 Cusiffrd}

General discussion 


\section{General discussion}

Cardiovascular disease (CVD) is the leading cause of death worldwide [1]. In low-income countries, CVD burden is steeply increasing at the moment. In high-income countries, CVD mortality has been decreasing over the last years due to improved pharmacological treatment, but the current rise in type 2 diabetes (T2DM) may compromise the achievements made in the past. Currently, low-grade inflammation and dysregulation of the immune system are of particular interest in cardiovascular research. The complement system is part of the innate immune system and regulates inflammation in liver, adipose tissue and in immune cells. A great body of in vitro and animal studies suggested a causal role of the complement system in different processes contributing to CVD. The complement network is classified into four main pathways of complement activation. Findings from animal studies implied that these different pathways may have distinct roles in the development of CVD [2-4]. However, it is currently unclear whether the different complement activation pathways are also in humans relevant in CVD. The present thesis therefore aimed to investigate the four main complement activation pathways in development of CVD using epidemiological study designs. This chapter summarizes the main findings of this thesis and discusses the most important methodological considerations of this approach. Furthermore, it presents scientific and public health implications of these findings and proposes directions for future research.

\section{Summary of the main findings}

Chapter 2 summarized previous observations on complement factors in human cardiometabolic disease. The majority of studies investigated C3, and showed positive associations of C3 with insulin resistance, liver dysfunction, the metabolic syndrome, T2DM and, inconsistently, with CVD. Furthermore, several large studies investigated mannosebinding lectin (MBL) in CVD, but yielded contradictory findings. Studies on other complement factors are scarce, and were mostly case-control studies, cross-sectional or were done in selected populations, such as in patients with T2DM.

Chapter 3 provided a more detailed overview on the mechanisms that may involve $\mathrm{C} 3$ and C3 activation in development of T2DM, CVD and in particular diabetic CVD. For example, C3 is a structural component of fibrin clots, where it increases clot solidity and resistance to lysis. The incorporation of C3 into clots appeared to be pronounced in patients with T2DM. Overall, the role of C3 in CVD may thus be modulated by other cardiovascular risk factors, such as hyperglycaemia.

In each of the Chapters 4-8, we present original data on the different complement activation pathways in CVD.

In Chapter 4, we investigated C3 and its activated product C3a in a cross-sectional analysis. We also examined whether a crucial cardiovascular risk factor, smoking, modulated the associations of C3 and C3a with CVD. Smoking was shown to activate C3 in several experimental studies [5-7]. C3 and C3a were both positively associated with low-grade 
inflammation. However, only C3a but not C3 was positively associated with markers of atherosclerosis. Associations with inflammation and atherosclerosis were not modulated by smoking, but we observed strong effect modification by smoking in associations with prevalent CVD. Both C3 and C3a were significantly associated with prevalent CVD, but only in the group of heavy smokers. Importantly, their associations with CVD in smokers were independent of each other, which suggests that distinct mechanisms may underlie these associations. To explore this, we conducted a post-hoc analysis and sub-classified CVD into atherothrombotic and other aetiology. In this analysis, C3 was exclusively associated with atherothrombotic events, while C3a was equally associated with CVD of atherothrombotic and of other aetiology. Overall, these findings imply that C3 and C3a reflect different mechanisms in CVD. C3 by itself does obviously not represent complement activation, and may contribute to CVD via other mechanisms, potentially via hypofibrinolysis, as discussed in Chapter 3.

In Chapter 5, we investigated products of terminal pathway activation C5a and sC5b-9 in a cross-sectional analysis. $\mathrm{C} 5 \mathrm{a}$ and $\mathrm{sC} 5 \mathrm{~b}-9$ were positively associated with low-grade inflammation and endothelial dysfunction. In contrast, C5a and sC5b-9 were not associated with markers of atherosclerosis or prevalent CVD. In the latter associations, the regression coefficients for markers of atherosclerosis were close to zero, and the ORs for CVD was close to one. This indicates that a lack of power is unlikely to explain these null-findings. Overall, systemic terminal complement activation may be involved in ongoing inflammatory processes and acute endothelial activation, but does not appear to be relevant in atherosclerosis and stable CVD.

In Chapter 6, we investigated three factors of the alternative pathway in a longitudinal analysis. We measured $\mathrm{Bb}$, which reflects current alternative pathway activation; factor $\mathrm{D}$ (FD), which is the rate-limiting protease in the activation step, and properdin. Properdin stabilizes the enzymatic complex of the alternative pathway and is thus an enhancer of alternative pathway activation. FD and $\mathrm{Bb}$ were positively associated with low-grade inflammation, and all three factors were positively associated with endothelial dysfunction. In the associations with incident CVD and cardiovascular events (CVE), the ORs of properdin, $\mathrm{FD}$ and $\mathrm{Bb}$ were all positive, and properdin was positively and significantly associated with incident CVE. Together, these observations suggest that the alternative pathway contributes to several processes of CVD and in particular to development of CVE. Properdin, FD and Bb were not associated with a marker of atherosclerosis, carotid intima-media thickness (cIMT). Obviously, the association with CVE may not be explained by a general role in atherosclerosis. The alternative pathway can be activated spontaneously in the vasculature, and is thought to be involved in platelet activation. Spontaneous activation of the alternative pathway may thus contribute to CVE via endothelial damage and, possibly, atherothrombosis. Furthermore, our findings indicate that especially a higher capacity to activate the alternative pathway as reflected by properdin, but not the current level of alternative pathway activation as reflected by $\mathrm{Bb}$, appears to be relevant in the development of CVE over time.

In chapter 7, we investigated three factors of the classical pathway in a longitudinal analysis. We measured the pattern-recognition factor $\mathrm{C} 1 \mathrm{q}$, the activation marker $\mathrm{C} 4 \mathrm{~d}$, and an inhibitor 
of classical pathway activation, C1-inhibitor. We observed a significant U-shaped association of C1q with incident CVD, and a similar, but non-significant association with CVE. This suggests that C1q may have protective as well as harmful effects in the development of CVD. Animal studies showed indeed a beneficial function of $\mathrm{C} 1 \mathrm{q}$ in atherosclerosis. However, $\mathrm{C} 1 \mathrm{q}$ was not associated with cIMT. C1q can also direct macrophage polarisation towards an antiinflammatory phenotype. Therefore, a protective role of $\mathrm{C} 1 \mathrm{q}$ in atherosclerosis could also involve effects on plaque phenotype, which is an aspect of atherosclerosis not reflected in cIMT. An adverse effect of C1q in CVD may be explained by its role in insulin resistance and metabolic inflammation. Observations on C4d were inconclusive, and C1-Inhibitor was not associated with any of the cardiovascular outcomes. Overall, these observations may imply that recognition of targets by $\mathrm{C} 1 \mathrm{q}$, but not downstream activation of the classical pathway is relevant in the development of CVD.

In chapter 8 , we investigated MBL, MBL-associated proteases (MASPs) and MBL-associated protein 44 (MAp44) of the lectin pathway in a longitudinal analysis. MBL can recognize abnormal structures and MASPs and MAp44 regulate complement activation. We observed a U-shaped association of MBL with low-grade inflammation, and observed that lower MBL was associated with higher cIMT. This suggests that low MBL may lead to more atherosclerosis and more low-grade inflammation, potentially due to impaired clearance of damaged tissues. On the other hand, high MBL may result in hyperresponsiveness to inflammatory triggers, and therefore also contribute to more low-grade inflammation. $\mathrm{MBL}$ can induce 'silent' clear-up processes without further complement activation, which may explain why high MBL was at the same time associated with more inflammation but a lower CIMT. MASP-1 and MASP-2, which mediate downstream complement activation upon MBL binding, were not associated with cardiovascular outcomes. Accordingly, the capacity to recognize targets via $\mathrm{MBL}$, but not the efficiency to translate this binding into downstream complement activation, may be relevant in inflammation and atherosclerosis. MASP-3 and MAp44, for which the roles in complement activation are not well-defined, were independently of MBL associated with endothelial dysfunction. MASP-3 can also cleave noncomplement proteins, therefore its association with endothelial dysfunction might be explained by functions independent of lectin pathway activation. Overall, our findings suggest that also the lectin pathway may have beneficial as well as adverse roles in the development of CVD. 


\section{Methodological considerations}

\subsection{Validity of determinants and outcomes}

\section{a) Validity of complement factors}

We measured systemic concentrations of complement factors assuming that they reflect their concentrations in relevant tissues. In the associations with endothelial dysfunction, this assumption may be fulfilled, because the endothelium is directly exposed to the circulation and to circulating complement factors. In the associations with low-grade inflammation and atherosclerosis, this assumption may be less feasible, because local complement concentrations in liver, adipose tissue and the vessel wall may be more relevant than systemic concentrations. In liver and in adipose tissue, local complement concentrations may be instrumental in the induction of inflammation, leading to release of inflammatory factors into the circulation. In the vessel wall, local complement concentrations may be relevant in the recognition of abnormal structures, attraction and activation of immune cells and in stimulation of smooth muscle cell proliferation. Local concentrations in liver, adipose tissue and the vessel wall may partly originate from diffusion of complement factors from systemic circulation [8-10], but may partly also depend on local production and local complement activation. Most complement factors can be produced in liver, adipose tissue and by immune cells infiltrated into the vessel wall (see chapter 2; $[3,11])$. For "inactive" complement factors with relatively high systemic concentrations such as C3 (see figure 1), the correlation between local and systemic concentrations may potentially be highest, because the capacity for local production may be unlikely to exceed diffusion from systemic circulation materially. In contrast, when systemic concentrations are relatively low, local production may have the potential to increase local concentrations substantially above systemic levels. Especially for the activated products such as $\mathrm{C} 5 \mathrm{a}$, local complement activation may increase local concentrations manifold above systemic levels. Higher local than systemic concentrations in relevant tissues were for example shown in synovial fluids from patients with rheumatoid arthritis [12-15]. In addition, local complement activation in liver and adipose tissue is thought to have mainly paracrine and autocrine effects [16-20], which also suggests that systemic concentrations may not fully represent concentrations that exert biological effects in inflammation and atherosclerosis in relevant tissues. Consequently, the associations described in this thesis most likely underestimate the relationship between complement factors and low-grade inflammation and atherosclerosis.

\section{b) Validity of outcomes}

We investigated low-grade inflammation, endothelial dysfunction and atherosclerosis because these are crucial pathophysiological processes of CVD. We assessed low-grade inflammation and endothelial dysfunction with biomarker scores, and used cIMT and anklearm blood pressure index (AAIx) as markers of atherosclerosis. Biomarkers of low-grade inflammation and endothelial dysfunction as well as cIMT and AAlx were shown to be associated with future CVD in several studies, which implies that they indeed reflect crucial processes of CVD [21-31]. Based on some limitations, these measures may however not 
perfectly represent the pathophysiological process of interest. For the measurement of lowgrade inflammation, no gold-standard has been established, and it is currently unclear whether systemic or local low-grade inflammation is most important in development of CVD. On the one hand, systemic low-grade inflammation is thought to induce pathophysiological processes in several organs and tissues [32], and studies that identified low-grade inflammation as a risk factor for CVD have almost always investigated systemic inflammation. Thus, measuring systemic inflammatory markers may be perfectly valid when inflammation is conceived as crucial risk factor for CVD. On the other hand, systemic low-grade inflammation originates from local inflammation in liver and adipose tissue [33-38], which suggests that measurement of local inflammation may be a more direct measure of the concept low-grade inflammation. Given that local, biopsy-proven inflammation in liver and adipose tissue was associated with systemic inflammation [39-42], measurement of circulating inflammatory markers may at least be a good reflection of local inflammation, in case that the latter is decisive in development of CVD. With regard to endothelial dysfunction, it should be noted that circulating markers secreted by a compromised endothelium are only indirect measures, while also direct, functional measurements exist, such as flow-mediated dilatation of peripheral arteries $[43,44]$. Circulating markers and functional measurements were shown to correlate [45], but may each reflect distinct aspects of endothelial (dys)function. Circulating markers are thought to reflect endothelial activation and endothelial permeability, while functional measures reflect the capacity for vasodilation [4650]. Therefore, the associations investigated in this thesis could cover only one aspect but not the full complexity of endothelial (dys)function. Also cIMT and AAlx as markers for atherosclerosis each reflect only one specific aspect of atherosclerosis. First, cIMT and AAIX reflect atherosclerosis in discrete parts of the vasculature. cIMT reflects atherosclerosis in the carotid arteries supplying the brain and AAlx reflects atherosclerosis in the lower limbs. This is likely a minor limitation, as atherosclerosis is a systemic disease and atherosclerosis in a single arterial site was shown to correlate with atherosclerosis in other vascular beds [51-55]. More importantly, cIMT represents thickening of the arterial wall and AAIx represents narrowing or occlusion of peripheral arterial vessels. However, atherosclerosis involves many more aspects, such as plaque burden, plaque phenotype, arterial remodelling and calcification [56-64]. Especially the measurement of plaque burden and coronary calcification is nowadays considered to be superior to the measurement of cIMT in order to assess the severity of atherosclerosis [56, 65-67].

The validity of the dichotomous outcomes CVD and CVE is very high, because here, the intention is to measure functional and clinically detectable impairments in the arterial vasculature. 


\subsection{Reliability}

\section{a) Biological variability}

The complement factors were determined in single measurement in fasted samples, assuming that these reflect their habitual concentrations. For most complement factors, little is known on their short-term variability, i.e. diurnal variability and day-to-day variability, and their long-term variability. Diurnal variability may originate from acute effects of meals, physical exercise or mood on systemic complement factors. Food-intake was observed to acutely affect plasma FD, C4, C3 and C3a, while Factor B and C5a were observed to be stable in the postprandial phase [68-75]. Acute exercise was observed to change plasma C1q, C3, $\mathrm{C} 3 \mathrm{a}, \mathrm{C} 5 \mathrm{a}$ and sC5b-9 [76-82]. Acute psychological stress was observed to change plasma $\mathrm{Bb}$, C3a and C5 [83]. Furthermore, for plasma C4, C3 and C3a, a circadian rhythm was observed [84], and also sleep deprivation was observed to change plasma C3 and C4 [85]. In addition, variability in plasma C3a and C1-inhibitor was observed during the menstrual cycle [86, 87]. These studies each only measured one or a few complement factors, and thus do not give a comprehensive picture on the biological variability of plasma complement factors. But together, they illustrate that there may be substantial biological variability in several complement factors. Suboptimal reflection of habitual levels in a single fasted sample may have resulted in random error, as all participants were measured under the same conditions. This suggests that many observed associations are likely to underestimate the true associations of complement factors with cardiovascular outcomes. In contrast, MBL and MASP-2 were in healthy individuals relatively stable during the day, were not influenced by acute exercise and $\mathrm{MBL}$ was reported to be stable over a period of six months or even 20 years $[88,89]$. For low-grade inflammation and endothelial dysfunction, random error due to biological variability was minimized by measuring several plasma markers and combining them into a score [90-92]. The reliability of the low-grade inflammation score may be slightly higher than reliability of the endothelial dysfunction score, because for low-grade inflammation eight (cross-sectional analyses) or six (longitudinal analyses) plasma inflammatory markers were pooled, while for endothelial dysfunction only four plasma markers were available.

\section{b) Measurement error in the laboratory measurements}

All laboratory measurements of complement factors and biomarkers of low-grade inflammation and endothelial dysfunction were performed in duplicate and blinded to participant characteristics in order to limit random and systematic measurement error, respectively. However, long-term storage and freeze-thaw cycles of samples may have introduced measurement error in the laboratory measurements, although the effect appears to have been minor.

All samples for the laboratory measurements had been stored at $-80^{\circ} \mathrm{C}$ in order to minimize the effects of sample storage $[93,94]$. Complement factors were only measured in the baseline samples, and we observed that absolute concentrations of all complement factors were within the same range as observed in previous studies (detailed discussion in the individual chapters). This suggests that the effect of sample storage on absolute 
concentrations of complement factors is most likely neglectable. With regard to biomarkers of low-grade inflammation and endothelial dysfunction, sample storage may have affected their absolute concentrations differently at baseline and at follow-up. Most biomarkers were measured paired, consequently the follow-up samples had been stored for $\approx 3-5$ years, while the baseline samples had been stored for $\approx 10-12$ years at the time of the measurements. C-reactive protein has been shown to be relatively stable during long-term storage [95], while other biomarkers may be less stable. For example, concentrations of interleukin- 6 and TNF $\alpha$ have been shown to decrease with longer sample-storage [96]. In that study [96], the greatest decline occurred between the second and the fourth year, which suggests that concentrations in samples stored for five years may have decreased to greater extent than concentrations in samples stored for three years. Consequently, at follow-up, sample storage time may be related to absolute biomarker concentrations. Together with the circumstance that in CODAM, relatively more patients with T2DM were included at the end of the inclusion procedure (see introduction), this may have resulted in a systematic measurement error, as storage time in the follow-up samples may be related to both absolute concentrations of biomarkers and T2DM. In contrast, at baseline, samples stored for ten years are unlikely to be substantially stronger affected from a storage effect than samples stored for twelve years, because with longer duration, storage effects may reach a plateau and may balance out over the samples. Thus, sample storage may only at follow-up have introduced a systematic error into the measurements of biomarkers of low-grade inflammation and endothelial dysfunction. Systematic measurement error may bias associations in either direction $[97,98]$. In case associations with low-grade inflammation or endothelial dysfunction differed between baseline and follow-up, associations at follow-up were weaker. Thus, even though a systematic measurement error might have affected the follow-up samples, this appears to have introduced bias towards the null.

In order to minimize the effect of freeze-thaw cycles, all activated products ( $C 4 d, B b, C 3 a$, C5a, sC5b-9) and also properdin, C3, C4 and MBL were measured in samples that had never been thawed before. Other complement factors were measured in samples that had been thawed maximally twice before, and for those measurements, tests had been performed beforehand to evaluate the effect of freeze-thaw cycles. In these tests, freeze-thaw cycles did not appear to affect the absolute concentrations measured. Biomarkers of low-grade inflammation and endothelial dysfunction were also measured in samples that had maximally been thawed twice before, and for several of those biomarkers (CRP, interleukin-6, sICAM1, sVCAM-1) with the exception of TNF $\alpha$, it has been shown that repeated freeze-thaw cycles may have a minor effect on the absolute concentrations [96, 99].

Lastly, it should be noted that none of these laboratory measurements are standardized; therefore the absolute concentrations of complement factors measured in our study (figure 1) may be slightly different from the true absolute concentrations. As this does not affect the ranking of the participants, this is unlikely to have affected the observed associations. 


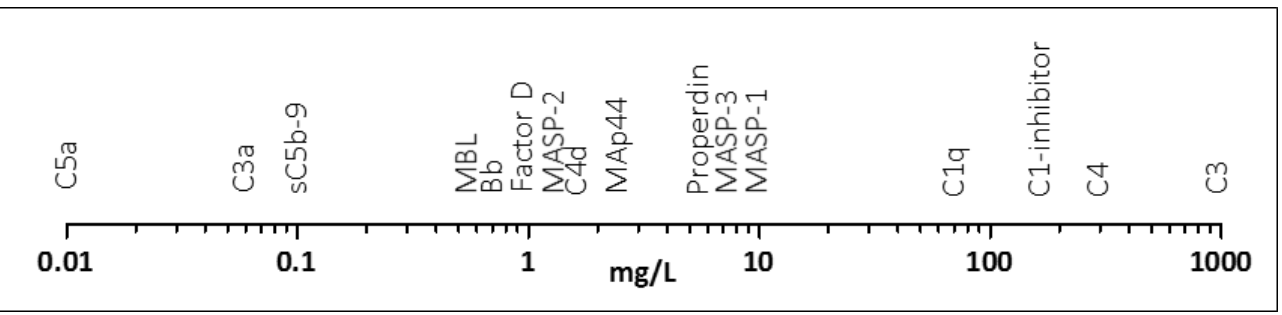

Figure 1: Median absolute systemic concentrations of complement factors in CODAM.

\subsection{Study design}

\section{a) Cross-sectional and longitudinal analyses}

The current thesis presents cross-sectional as well as longitudinal analyses. Longitudinal analyses allow to investigate temporality between associations, which is considered to provide better evidence for causality [100]. However, longitudinal analyses may also have disadvantages related to typical problems with the follow-up procedure. In CODAM, $14 \%$ of participants were lost-to-follow up, and those participants also had a slightly worse cardiometabolic profile than participants who attended the follow-up measurements. This indicates that a selective drop-out of unhealthier participants has occurred, which may have resulted in a smaller chance to detect associations. For example, participants who experienced a lethal cardiovascular event were not included in the follow-up sample, which means that the most severe incident CVD cases were not included in the analyses. Thus, despite advantages of longitudinal analyses in terms of causality, the issue of selection bias may have resulted in underestimation of several associations.

\section{b) Confounding and overadjustment}

Confounding is a source of bias in the estimation of causal effects. A confounder is a variable that creates a spurious relation between the exposure and the outcome [101]. Traditionally, a confounder is defined as being associated with the outcome, being associated with the exposure, but not being a consequence of the exposure (thus not being a mediator). At first glance, the identification of confounders in the research question of interest appears thus to be straightforward. However, in practice, the issue of confounding is highly complex, making it difficult to categorize variables unequivocally as confounders or mediators. First of all, in biomedical research, bidirectional relations and vicious circles between an exposure and a potential confounder may exist. For example, adipose tissue secretes complement factors, but several complement factors were in animal studies shown to be causally linked to obesity, suggesting a bidirectional relation. Second, current views how to deal with common causes of exposure and disease are conflicting. For example, adipose tissue contributes to CVD and also secretes complement factors, therefore obesity is a common cause of circulating complement factors and of CVD. Conservatively, a common cause of exposure and disease is clearly categorised as confounder [102]. As opposed to this, others advocate, that when the exposure is caused by the confounder, but mediates the effect of the potential 
confounder on the outcome, adjusting for that variable would be incorrect [103]. According to this view, adjustment for obesity may be inappropriate when it is assumed that secretion of complement factors partly explains how obesity contributes to CVD. Lastly, it has now become clear, that the extent of measurement error in exposure and covariates has profound effects on the results [104, 105]. Simulation studies showed that adjustment for covariates that are more precisely measured than the exposure of interest may result in underestimation of the exposure effect [106], and in overestimation of the covariate effect. In this thesis, we adjusted all analyses for the most important cardiovascular risk factors. Most of these potentially confounding variables are likely to be measured with higher precision than the complement factors. For example, obesity or use of medication is measured more reliably because they feature basically no short-term variability. Blood pressure or blood lipids may have similar biological variability to complement factors, but the measurement procedure may be more reliable because these are clinical measures carried out according to standardized procedures. Together, these reflections aim to illustrate that any 'true' association may not be described with one definite statistical model. Several of the variables we adjusted for may be confounders and mediators at the same time, therefore, the fully adjusted models in this thesis may to some extent be overadjusted [107]. Furthermore, a higher degree of measurement error in complement factors than in covariates may have resulted in underestimation of associations of complement factors with cardiovascular outcomes. These issues are fundamentally relevant for the interpretation of the current results, because several of the associations presented in this thesis were just significant or borderline significant. Vagueness in the concept of confounding and practical problems in the implementation of adjustments should be considered when judging the overall relevance of our findings. On the other hand, the possibility of residual confounding can never be excluded in observational studies [108].

\section{c) Causality}

Proof of causality was not the primary aim of the current thesis. As described above, our results may to some extent be affected by several forms of bias. Furthermore, reverse causation may have occurred in the cross-sectional associations with CVD. However, no study design may exist that is completely free of bias and is perfectly generalizable to the population of interest. Experimental animal and in vitro studies showed a causal role of the complement system in several processes of CVD, but may not be fully representative for the complex disease process in humans. Our study aimed to explore whether these mechanisms implicated in basic science studies are relevant in humans. Together with findings from experimental studies, the current observational evidence strengthens the concept that the complement system is causally involved in CVD.

\section{d) Generalizability}

The CODAM study includes individuals with an increased cardiovascular risk. Therefore, the effect sizes may be different in populations with other distributions of complement factors and cardiovascular outcomes [109]. However, the aim of this thesis was not to estimate the predictive effect of complement factors, but to explore their role in the aetiology of CVD. The 
pathophysiological concept that the complement system is involved in CVD does not rely on the study population to be representative for another population [110]. Aside from that, our findings can be generalized to the large group of Western individuals who have one or more cardiovascular risk factor.

\section{Overall interpretation of results}

\subsection{Relevance of the different complement pathways in human CVD}

Taken together, the findings presented in the current thesis sketch the following overall picture of the role of the complement system in human CVD (table 1): First, activation of the alternative pathway may be harmful in the development of CVD and in particular CVE. All measured components of the alternative pathway correlated positively with adverse cardiovascular outcomes, and higher levels of properdin were independently associated with future CVE. Second, the classical and the lectin pathway may have beneficial as well as adverse roles in development of CVD. Both high and low levels of the pattern recognition molecules $\mathrm{C} 1 \mathrm{q}$ and $\mathrm{MBL}$ correlated with worse cardiovascular outcomes. Factors that reflect the level or the capacity for classical and lectin pathway activation, C4d and C1-inhibitor, were not consistently associated with cardiovascular outcomes. This also supports the concept that classical and lectin pathway activation is not exclusively harmful in CVD. Third, systemic activation of the terminal pathway may be adverse with regard to low-grade inflammation and endothelial dysfunction, but does not appear to be relevant in CVD. Lastly, the function of the C3-C3a axis in CVD appears to be complex. Higher levels of C3 and C3a may be adverse with regard to CVD, but appear to reflect distinct molecular mechanisms.

\subsection{Relevance of the individual complement factors in human CVD}

In addition, the findings of the current thesis reveal three further important aspects of the role of complement factors in CVD. First, we observed that different components of each pathway did not show similar associations with cardiovascular outcomes. For example, associations of MBL and MASPs as well as associations of C1q and C1-inhibtor with cardiovascular outcomes were substantially different. This may be explained by the concept that they reflect distinct molecular mechanisms: For example, C1q may rather represent the capacity to recognize and opsonize targets, while higher c1-inhibitor may reflect the potential to inhibit complement activation after binding of $\mathrm{C} 1 \mathrm{q}$ to targets. A higher capacity for target recognition may however not necessarily lead to more complement activation, and therefore not critically depend on an inhibitor to exert effects. Second, higher concentrations of the pattern recognition factors $\mathrm{C} 1 \mathrm{q}$ and $\mathrm{MBL}$ were not correlated with the activation product of the classical and the lectin pathway $\mathrm{C} 4 \mathrm{~d}$. This may indicate, that associations of C1q and MBL with CVD and CIMT, respectively, may arise for partly from their function as pattern recognition factors and their capacity to opsonize targets without further complement activation. Alternatively, this may be explained by the third observation: all activated products correlated positively with low-grade inflammation, but most of them 
(C4d, Bb, C5a, sC5b-9, with the exception of C3a) were not or only weakly associated with stable outcomes such as CVD and cIMT. This suggests that systemic concentrations of most activated products reflect dynamic processes such as low-grade inflammation, but may insufficiently reflect complement activation over time or local concentrations in relevant tissues.

Table 1: Overview on the fully adjusted associations of complement factors with cardiovascular outcomes in the whole study population

\begin{tabular}{|c|c|c|c|c|c|c|c|}
\hline \multicolumn{2}{|c|}{$\begin{array}{l}\text { complement pathway } \\
\text { factor }\end{array}$} & \multirow{2}{*}{$\begin{array}{l}\text { function } \\
\text { pattern- } \\
\text { recognition }\end{array}$} & \multicolumn{3}{|c|}{$\begin{array}{l}\text { low-grade endothelial cIMT } \\
\text { inflammation dysfunction }\end{array}$} & \multirow{2}{*}{$\begin{array}{l}\begin{array}{l}\text { incident } \\
\text { CVD }\end{array} \\
\cup \cup\end{array}$} & \multirow{2}{*}{$\begin{array}{l}\begin{array}{l}\text { incident } \\
\text { CVE }\end{array} \\
U\end{array}$} \\
\hline $\mathrm{C} 1 \mathrm{q}$ & classical & & + & ++ & 0 & & \\
\hline \multicolumn{3}{|c|}{ C1-Inhibitor classical/lectin inhibitor } & 0 & 0 & 0 & 0 & 0 \\
\hline C4d & classical/lectin & $\begin{array}{l}\text { activation } \\
\text { marker }\end{array}$ & ○ & 0 & 0 & 0 & O \\
\hline $\mathrm{MBL}$ & lectin & $\begin{array}{l}\text { pattern- } \\
\text { recognition }\end{array}$ & $\cup \cup$ & 0 & -- & - & - \\
\hline MASP-1 & lectin & protease & 0 & 0 & ○ & 0 & 一 \\
\hline MASP-2 & lectin & protease & 0 & 0 & ○ & 0 & ○ \\
\hline MASP-3 & lectin & $\begin{array}{l}\text { regulatory } \\
\text { functions? }\end{array}$ & O & ++ & 0 & + & + \\
\hline MAp44 & lectin & $\begin{array}{l}\text { regulatory } \\
\text { functions? }\end{array}$ & ○ & ++ & 0 & 0 & ○ \\
\hline Properdin & alternative & $\begin{array}{l}\text { enhancer } \\
\text { initiator? }\end{array}$ & + & ++ & O & O & +++ \\
\hline Factor D & alternative & protease & +++ & +++ & 0 & + & + \\
\hline $\mathrm{Bb}$ & alternative & $\begin{array}{l}\text { activation } \\
\text { marker }\end{array}$ & ++ & ++ & ○ & + & ○ \\
\hline C3 & $\begin{array}{l}\text { convergence } \\
\text { alternative }\end{array}$ & protease & +++ & ++ & O & O & + \\
\hline C3a & convergence & anaphylatoxin & +++ & + & +++ & + & 0 \\
\hline C5a & terminal & anaphylatoxin & +++ & ++ & 0 & O & ○ \\
\hline sC5b-9 & terminal & $\begin{array}{l}\text { activation } \\
\text { marker }\end{array}$ & +++ & +++ & O & ○ & 0 \\
\hline $\begin{array}{l}\text { Legend: On } \\
+++ \text { signs de } \\
\text { relatively st } \\
\text { significant } \\
\text { association; } \\
\text { completion } \\
\text { described ir } \\
0.15] \text {; incid }\end{array}$ & $\begin{array}{l}\text { - or + sign de } \\
\text { note a negative } \\
\text { rong negative or } \\
\text { battern of an as } \\
\text { in grey: to prov } \\
\text { of the current } \\
\text { chapters } 7 \text { anc } \\
\text { nt CVD, C3a: OP }\end{array}$ & $\begin{array}{l}\text { enotes a negati } \\
\text { e or positive, sig } \\
\text { r positive, signifi } \\
\text { ssociation; two } \\
\text { vide a comprehe } \\
\text { table but were } \\
\text { d 8), endothelia } \\
R=2.07 \text { [0.90; } 4 .\end{array}$ & $\begin{array}{l}\text { ve or posit } \\
\text { gnificant as } \\
\text { ficant assoc } \\
\cup \text { sig } \\
\text { ensive pict } \\
\text { e not pres } \\
\text { al dysfunct } \\
.76] \text {, incide }\end{array}$ & $\begin{array}{l}\text { but non } \\
\text { iation; th } \\
\text { ion; one } \\
\text { denote a } \\
\text {, the foll } \\
\text { ed in ch } \\
\text { CB: } \beta=C \\
\text { CVE, C3a: }\end{array}$ & $\begin{array}{l}\text { nificant } \\
\text { ign deno } \\
\text { shaped, } \\
\text { ng associ } \\
\text { ers 4-8: } \\
{[0.04 ; 0}\end{array}$ & $\begin{array}{l}\text { ssociation, } \\
\text { or }+++ \text { s } \\
\text { tes a U-sha } \\
\text { jignificant } \\
\text { ations wer } \\
\text { ully adjust } \\
\text { 22]; C3a: } \\
\text { 37; } 3.80] \text {. }\end{array}$ & $\begin{array}{l}\text { gwo - - } \\
\text { gns denote } \\
\text { ed, but nor } \\
\text { attern of a } \\
\text { analysed fo } \\
d \text { model (a } \\
=0.08[-0.0\end{array}$ \\
\hline
\end{tabular}




\subsection{Comparison with previous studies}

\section{a) Human studies}

As summarized in chapter 2, C3 and MBL have been measured in several cohorts, while human studies on other complement factors are scarce. We observed that C3 was independently associated with prevalent CVD, but not with future CVD. Other studies consistently observed positive associations with prevalent CVD, while in the few prospective studies, the association of C3 with CVD was not always independent of confounders [111113]. This suggests that associations of C3 with prevalent CVD may partly arise from reverse causation, or may be mediated by covariates or unmeasured variables strongly related to covariates. Our observations revealed that activation of C3, i.e. reflected by C3a, did not explain associations of C3. Potentially, associations of C3 with CVD may partly arise from effects beyond complement activation. Interestingly, C3 was recently shown to bind unspecifically to several plasma proteins via its reactive thioester group, which can be exposed spontaneously $[114,115]$. Considering the relatively high plasma concentration of C3, this mechanism may have interesting yet unresolved functions in CVD. Next, we observed that MBL may have beneficial as well as adverse effects in low-grade inflammation and atherosclerosis. This may explain why previous studies have been contradictory, and observed either positive or inverse associations with CVD. Regarding complement factors other than $\mathrm{C3}$ or $\mathrm{MBL}$, only one large prospective study is available on FD. FD was in a large cohort of men associated with risk of stroke, but not with risk of coronary heart disease [116, 117]. This is in line with our findings, as it also suggests that the alternative pathway may in particular contribute to atherothrombosis. We measured several alternative pathway factors and observed the strongest association between properdin and CVE. With regard to other complement factors, this is the first longitudinal study on Bb, C1q, C1-inhibitor, C4d, MASPs and MAp44 in CVD. Therefore, comparison with previous studies is not possible.

\section{b) Animal and in vitro studies}

Our findings confirm that several causal mechanisms suggested by experimental studies are relevant in humans. Our findings on the classical and the lectin pathway are largely in agreement with animal studies, which already indicated protective as well as harmful functions of $\mathrm{C} 1 \mathrm{q}$ and $\mathrm{MBL}$ in different processes of $\mathrm{CVD}$. Protective functions of $\mathrm{C} 1 \mathrm{q}$ and $\mathrm{MBL}$ were observed in mice models that mimic the early stages of atherosclerosis (LDL-receptor knockout mice, $\mathrm{LDLR}^{--}$) [118]. Mice genetically deficient in C1q or $\mathrm{MBL}$ had more severe atherosclerosis and an impaired clearance of apoptotic cells [119-121]. Harmful functions in CVD may be explained differently for C1q and MBL. C1q appears to have an adverse role in metabolism, as mice deficient in $\mathrm{C} 1 \mathrm{q}$ developed less insulin resistance and glucose intolerance on a high-fat diet [122]. For MBL, a harmful role was shown in mice models that simulate later stages of CVD, when hyperglycaemia or oxidative stress advanced vascular tissue damage $[123,124]$. In mice, MBL had thus a beneficial function in lipid-induced atherosclerosis, but had an adverse function in hyperglycaemic vascular damage.

Our findings on the alternative pathway are in line with the available mice models on atherosclerosis; however they lead to different conclusions regarding the overall relevance 
in CVD. In LDLR ${ }^{-/}$mice, deficiency of properdin or Factor B did not have consistent effects on atherosclerosis [125-127], which would imply that the alternative pathway is not crucially relevant in CVD. In contrast, our findings suggest that in humans, the alternative pathway is highly relevant in CVD. In line with the mice models, we did not observe an association of the alternative pathway with atherosclerosis. However, we observed that the alternative pathway was in particular associated with CVE, which may be explained by a role in atherothrombosis. In agreement, deficiency of Factor B resulted in less microvascular thrombosis in a mouse model of haemolytic uraemic syndrome, although the translatability to arterial thrombosis may be limited [128]. Several in vitro studies also suggested functions of the alternative pathway in endothelial activation, endothelial release of procoagulant factors and platelet activation [129-134]. In line with that, we observed a relatively strong association of all measured alternative pathway components with endothelial dysfunction (regression coefficients up to twice as high than for example of MASP-3 or MAp44). Our findings thus support the large body of in vitro studies that implicated the alternative pathway in coagulation. Furthermore, they illustrate that mice models only cover very specific aspects in the cardiovascular disease process, and that studies in a single model such as $\mathrm{LDLR}^{-/}$are limited to appraise the relevance of a certain factor in the complex development of human CVD.

Our findings on the terminal pathway are not in line with the majority of experimental studies. In in vitro studies, C5a appeared to be the most potent anaphylatoxin [135-137], which would suggest that $\mathrm{C} 5 \mathrm{a}$ is also most crucial in CVD. Furthermore, in mice models of atherosclerosis, deficiency of CD59, which protects cells from terminal pathway attack, resulted consistently in more atherosclerosis [138-141]. In line with an adverse role of terminal pathway activation, deficiency of C6 itself or inhibition of the C5a-receptor in mice decreased atherosclerosis $[138,142]$. We did however observe that terminal complement activation was clearly not associated with atherosclerosis, and also not with CVD. Only one animal study is in line with our findings, and did not observe an effect of C5 deficiency on atherosclerosis [143]. Importantly, systemic levels of terminal pathway activation may not reflect local and potentially more crucial effects inside the vessel wall. Nevertheless, our findings indicate that systemic activation of the terminal pathway is in human CVD less relevant as one would infer at first glance when reviewing data from animal studies.

Our findings on the C3-C3a axis are in the total picture consistent with experimental evidence from basic science, although they are partly in contrast with individual observations. Overall, animal studies already indicated that C3 and C3a may contribute to CVD via different mechanisms, and may have different roles in atherosclerosis and thrombosis. In mice models of atherosclerosis, effects of $\mathrm{C} 3$ deficiency were not consonant with effects of C3a-receptor deficiency: deficiency of the C3a-receptor decreased atherosclerosis [144], which is in line with the positive association of C3a with atherosclerosis observed in our study. Deficiency of C3 however resulted in more atherosclerosis, which would imply a beneficial function of C3 in atherosclerosis $[126,145]$. We did clearly observe that C3 was not associated with atherosclerosis, and our study suggested a harmful but not a beneficial role of C3 in other processes contributing to CVD. This contradiction may be explained by the fact that 
atherosclerosis in these mice models is excessively driven by dyslipidaemia and that C3 was repeatedly implicated in lipid metabolism. In mice, C3 may thus have a beneficial effect on atherosclerosis partly via lipid metabolism, while in humans, beneficial effects on lipid metabolism may be of minor relevance. We did observe that C3 was particularly associated with atherothrombotic events, which is in line with a study that observed delayed thrombosis in C3 deficient mice [146].

Overall, our findings from a human cohort substantiate a large part of animal and in vitro data, but also suggest that some pathways may in humans be more relevant (alternative pathway), less relevant (terminal pathway) or relevant in other aspects of CVD (C3) than anticipated from available mice models on atherosclerosis.

\section{Implications and future directions}

CVD is the most common cause of death worldwide [1], and better understanding of mechanisms contributing to CVD is needed in order to decrease the burden of CVD. This thesis showed that concentrations of complement factors in the circulation were associated with CVD as well as with low-grade inflammation, endothelial dysfunction and atherosclerosis. This suggests that the complement system may be involved in the development of CVD. Importantly, the different pathways of complement activation may play different roles. Especially the alternative pathway, which can be activated spontaneously in the circulation, appeared to have harmful effects, while the classical and the lectin pathway appeared to have harmful but also beneficial effects. These findings have several scientific and also some clinical implications. First of all, our study provided for the first time a comprehensive evaluation of the complement system in CVD in humans. The present findings substantiated data from animal and in vitro studies and gave also some new insights. For example, the alternative pathway did not increase atherosclerosis in mice models [126, 127], but appeared to be highly relevant in human CVD. Future studies should investigate in detail, which factors enhance spontaneous activation of the alternative pathway in the circulation. From a clinical point of view, in particular the enhancer of alternative pathway activation properdin may in the future be used as prognostic marker to identify individuals with an increased cardiovascular risk. The associations of properdin with incident CVE were independent of C3 and of low-grade inflammation, which suggests that properdin reflects indeed a novel aspect in the aetiology of CVD. Next, our findings on the lectin pathway may be especially relevant in the aetiology of CVD among other ethnicities. We observed that lower MBL levels were associated with more atherosclerosis. Genotypes resulting in low circulating $\mathrm{MBL}$ levels are more prevalent in African and South American populations [147-149], therefore low MBL may partly explain the observation that black race is associated with more atherosclerosis compared to white race [150, 151]. However, pharmacological targeting of the complement system in CVD is unlikely in the near future. The complement system has diverse roles in many physiological processes; and pharmacological therapy may not be successful unless finely adjusted modulation with strictly site-targeted, controlled drug delivery is available. Strategies to target complement in acute myocardial infarction have so far been unsuccessful and were discontinued [152, 
153]. On the other hand, a healthy diet, physical activity and commonly prescribed drugs may have beneficial effects on systemic complement factors, as described in chapter 2 .

In the future, our findings need of course replication in larger studies. However, also larger studies will suffer from similar limitations as our study regarding validity, reliability, and confounding/overadjustment. In order to further elucidate the role of complement in the aetiology of CVD, studies applying the principles of Mendelian randomization can add valuable information. In the Mendelian randomization approach, genetic variants that cause a change in concentration, activity or function in the respective protein are investigated as reflection of lifelong exposure. This approach limits bias by confounding as well as measurement error, and is considered superior to indicate a causal relationship. For most complement factors, already several polymorphisms are known that affect protein concentration or function [154-157]. Furthermore, several cases of genetic deficiency in for example C1, C4, C5, C6, C7, C8, C9 or Factor H have been reported [158-169]. In case that deficiencies did not lead to severe orphan diseases, their effects have so far been investigated mainly with regard to infectious diseases. In the future, polymorphisms should be characterized for their molecular effects and subsequently be merged in high- and lowactivity complotypes. Targeted investigation of complotypes in large GWAS databases combined with better characterization of cases with complement deficiencies may provide a deeper insight into the role of complement in CVD. 


\section{References}

1. World Health Organization. Global status report on non-communicable diseases 2014. [cited 7 February 2016]; retrieved from http://apps.who.int/iris/bitstream/10665/148114/1/9789241564854 eng.pdf.

2. Francescut L, Steiner T, Byrne S, Cianflone K, Francis S, Stover C. The role of complement in the development and manifestation of murine atherogenic inflammation: novel avenues. J. Innate Immun., 2012. 4: 260-72.

3. Oksjoki R, Kovanen PT, Meri S, Pentikainen MO. Function and regulation of the complement system in cardiovascular diseases. Front. Biosci., 2007. 12: 4696-708.

4. Speidl WS, Kastl SP, Huber K, Wojta J. Complement in atherosclerosis: friend or foe? J. Thromb. Haemost., 2011. 9: 428-40.

5. Kew RR, Ghebrehiwet B, Janoff A. Characterization of the third component of complement (C3) after activation by cigarette smoke. Clin. Immunol. Immunopathol., 1987. 44: 248-58.

6. Yin W, Ghebrehiwet B, Weksler B, Peerschke El. Regulated complement deposition on the surface of human endothelial cells: effect of tobacco smoke and shear stress. Thromb. Res., 2008. 122: 221-8.

7. Perricone R, de Carolis C, de Sanctis G, Fontana L. Complement activation by cigarette smoke condensate and tobacco infusion. Arch. Environ. Health, 1983. 38: 176-9.

8. Smith EB, Staples EM. Plasma protein concentrations in interstitial fluid from human aortas. Proc. R. Soc. Lond. B Biol. Sci., 1982. 217: 59-75.

9. Bent-Hansen L. Whole body capillary exchange of albumin. Acta Physiol. Scand. Suppl., 1991. 603: 5-10.

10. Taylor DG, Bert JL, Bowen BD. A mathematical model of interstitial transport. I. Theory. Microvasc. Res., 1990. 39: 253-78.

11. Morgan BP, Gasque P. Extrahepatic complement biosynthesis: where, when and why? Clin. Exp. Immunol., 1997. 107: 1-7.

12. Morgan BP, Daniels RH, Williams BD. Measurement of terminal complement complexes in rheumatoid arthritis. Clin. Exp. Immunol., 1988. 73: 473-8.

13. Miller JJ, 3rd, Olds LC, Huene DB. Complement activation products and factors influencing phagocyte migration in synovial fluids from children with chronic arthritis. Clin. Exp. Rheumatol., 1986. 4: 53-6.

14. Oleesky DA, Daniels RH, Williams BD, Amos N, Morgan BP. Terminal complement complexes and C1/C1 inhibitor complexes in rheumatoid arthritis and other arthritic conditions. Clin. Exp. Immunol., 1991. 84: 250-5.

15. Bengtsson A, Bengtson JP, Rydenhag A, Roxvall L, Heideman M. Accumulation of anaphylatoxins and terminal complement complexes in inflammatory fluids. J. Intern. Med., 1990. 228: 173-6.

16. Mathern DR, Heeger PS. Molecules Great and Small: The Complement System. Clin. J. Am. Soc. Nephrol., 2015. 10: $1636-50$.

17. Puschel GP, Oppermann M, Muschol W, Gotze O, Jungermann K. Increase of glucose and lactate output and decrease of flow by human anaphylatoxin C3a but not C5a in perfused rat liver. FEBS Lett., 1989. 243: 83-7.

18. Havel PJ. Control of energy homeostasis and insulin action by adipocyte hormones: leptin, acylation stimulating protein, and adiponectin. Curr. Opin. Lipidol., 2002. 13: 51-9.

19. Czermak BJ, Sarma V, Bless NM, Schmal H, Friedl HP, Ward PA. In vitro and in vivo dependency of chemokine generation on C5a and TNF-alpha. J. Immunol., 1999. 162: 2321-5.

20. Zhou $W$. The new face of anaphylatoxins in immune regulation. Immunobiology, 2012. 217: 225-34.

21. van den Oord SC, Sijbrands EJ, ten Kate GL, van Klaveren D, van Domburg RT, van der Steen AF, Schinkel AF. Carotid intima-media thickness for cardiovascular risk assessment: systematic review and meta-analysis. Atherosclerosis, 2013. 228: 1-11.

22. Eikendal AL, Groenewegen KA, Anderson TJ, Britton AR, Engstrom G, Evans GW, de Graaf J, Grobbee DE, Hedblad B, Holewijn S, Ikeda A, Kitagawa K, Kitamura A, Lonn EM, Lorenz MW, Mathiesen EB, Nijpels G, Dekker JM, Okazaki S, O'Leary DH, Polak JF, Price JF, Robertson C, Rembold CM, Rosvall M, Rundek T, Salonen JT, Sitzer M, Stehouwer CD, Hoefer IE, Peters SA, Bots ML, den Ruijter HM. Common carotid intima-media thickness relates to cardiovascular events in adults aged <45 years. Hypertension, 2015. 65: 707-13.

23. Criqui $\mathrm{MH}, \mathrm{McClelland} \mathrm{RL}$, McDermott MM, Allison MA, Blumenthal RS, Aboyans V, Ix JH, Burke GL, Liu K, Shea $\mathrm{S}$. The ankle-brachial index and incident cardiovascular events in the MESA (Multi-Ethnic Study of Atherosclerosis). J. Am. Coll. Cardiol., 2010. 56: 1506-12.

24. Aboyans V, Criqui MH, Abraham P, Allison MA, Creager MA, Diehm C, Fowkes FG, Hiatt WR, Jonsson B, Lacroix P, Marin B, McDermott MM, Norgren L, Pande RL, Preux PM, Stoffers HE, Treat-Jacobson D. Measurement and interpretation of the ankle-brachial index: a scientific statement from the American Heart Association. Circulation, 2012. 126: 2890-909.

25. Luc G, Arveiler D, Evans A, Amouyel P, Ferrieres J, Bard JM, Elkhalil L, Fruchart JC, Ducimetiere P. Circulating soluble adhesion molecules ICAM-1 and VCAM-1 and incident coronary heart disease: the PRIME Study. Atherosclerosis, 2003. 170: 169-76. 
26. Ridker PM, Rifai N, Stampfer MJ, Hennekens $\mathrm{CH}$. Plasma concentration of interleukin- 6 and the risk of future myocardial infarction among apparently healthy men. Circulation, 2000. 101: 1767-72.

27. Ridker PM, Hennekens CH, Buring JE, Rifai N. C-reactive protein and other markers of inflammation in the prediction of cardiovascular disease in women. N. Engl. J. Med., 2000. 342: 836-43.

28. Jenny NS, Arnold AM, Kuller LH, Sharrett AR, Fried LP, Psaty BM, Tracy RP. Soluble intracellular adhesion molecule-1 is associated with cardiovascular disease risk and mortality in older adults. J. Thromb. Haemost., 2006. 4: 107-13.

29. Morange PE, Simon C, Alessi MC, Luc G, Arveiler D, Ferrieres J, Amouyel P, Evans A, Ducimetiere P, JuhanVague I. Endothelial cell markers and the risk of coronary heart disease: the Prospective Epidemiological Study of Myocardial Infarction (PRIME) study. Circulation, 2004. 109: 1343-8.

30. Empana JP, Sykes DH, Luc G, Juhan-Vague I, Arveiler D, Ferrieres J, Amouyel P, Bingham A, Montaye M, Ruidavets JB, Haas B, Evans A, Jouven X, Ducimetiere P. Contributions of depressive mood and circulating inflammatory markers to coronary heart disease in healthy European men: the Prospective Epidemiological Study of Myocardial Infarction (PRIME). Circulation, 2005. 111: 2299-305.

31. Blankenberg S, Rupprecht HJ, Bickel C, Peetz D, Hafner G, Tiret L, Meyer J. Circulating cell adhesion molecules and death in patients with coronary artery disease. Circulation, 2001. 104: 1336-42.

32. Lumeng CN, Saltiel AR. Inflammatory links between obesity and metabolic disease. J. Clin. Invest., 2011. 121: 2111-7.

33. Perseghin G. The role of non-alcoholic fatty liver disease in cardiovascular disease. Dig. Dis., 2010. 28: $210-3$.

34. Cai D, Yuan M, Frantz DF, Melendez PA, Hansen L, Lee J, Shoelson SE. Local and systemic insulin resistance resulting from hepatic activation of IKK-beta and NF-kappaB. Nat. Med., 2005. 11: 183-90.

35. Trayhurn P, Wood IS. Adipokines: inflammation and the pleiotropic role of white adipose tissue. Br. J. Nutr., 2004. 92: 347-55.

36. Lee J. Adipose tissue macrophages in the development of obesity-induced inflammation, insulin resistance and type 2 diabetes. Arch. Pharm. Res., 2013. 36: 208-22.

37. Maury E, Brichard SM. Adipokine dysregulation, adipose tissue inflammation and metabolic syndrome. Mol. Cell. Endocrinol., 2010. 314: 1-16.

38. Mohamed-Ali V, Goodrick S, Rawesh A, Katz DR, Miles JM, Yudkin JS, Klein S, Coppack SW. Subcutaneous adipose tissue releases interleukin-6, but not tumor necrosis factor-alpha, in vivo. J. Clin. Endocrinol. Metab., 1997. 82: 4196-200.

39. Haukeland JW, Damas JK, Konopski Z, Loberg EM, Haaland T, Goverud I, Torjesen PA, Birkeland K, Bjoro K, Aukrust P. Systemic inflammation in nonalcoholic fatty liver disease is characterized by elevated levels of CCL2. J. Hepatol., 2006. 44: 1167-74.

40. Targher G. Relationship between high-sensitivity C-reactive protein levels and liver histology in subjects with non-alcoholic fatty liver disease. J. Hepatol., 2006. 45: 879-81.

41. Yoneda M, Mawatari H, Fujita K, lida H, Yonemitsu K, Kato S, Takahashi H, Kirikoshi H, Inamori M, Nozaki $Y$, Abe Y, Kubota K, Saito S, Iwasaki T, Terauchi Y, Togo S, Maeyama S, Nakajima A. High-sensitivity C-reactive protein is an independent clinical feature of nonalcoholic steatohepatitis (NASH) and also of the severity of fibrosis in NASH. J. Gastroenterol., 2007. 42: 573-82.

42. McLaughlin T, Liu LF, Lamendola C, Shen L, Morton J, Rivas H, Winer D, Tolentino L, Choi O, Zhang H, Hui Yen Chng $\mathrm{M}$, Engleman $\mathrm{E}$. T-cell profile in adipose tissue is associated with insulin resistance and systemic inflammation in humans. Arterioscler. Thromb. Vasc. Biol., 2014. 34: 2637-43.

43. Lekakis J, Abraham P, Balbarini A, Blann A, Boulanger CM, Cockcroft J, Cosentino F, Deanfield J, Gallino A, Ikonomidis I, Kremastinos D, Landmesser U, Protogerou A, Stefanadis C, Tousoulis D, Vassalli G, Vink H, Werner N, Wilkinson I, Vlachopoulos C. Methods for evaluating endothelial function: a position statement from the European Society of Cardiology Working Group on Peripheral Circulation. Eur. J. Cardiovasc. Prev. Rehabil., 2011. 18: 775-89.

44. Stoner L, Tarrant MA, Fryer S, Faulkner J. How should flow-mediated dilation be normalized to its stimulus? Clin. Physiol. Funct. Imaging, 2013. 33: 75-8.

45. Chong AY, Blann AD, Patel J, Freestone B, Hughes E, Lip GY. Endothelial dysfunction and damage in congestive heart failure: relation of flow-mediated dilation to circulating endothelial cells, plasma indexes of endothelial damage, and brain natriuretic peptide. Circulation, 2004. 110: 1794-8.

46. Deanfield J, Donald A, Ferri C, Giannattasio C, Halcox J, Halligan S, Lerman A, Mancia G, Oliver JJ, Pessina AC, Rizzoni D, Rossi GP, Salvetti A, Schiffrin EL, Taddei S, Webb DJ. Endothelial function and dysfunction. Part I: Methodological issues for assessment in the different vascular beds: a statement by the Working Group on Endothelin and Endothelial Factors of the European Society of Hypertension. J. Hypertens., 2005. 23: 7-17.

47. Constans J, Conri C. Circulating markers of endothelial function in cardiovascular disease. Clin. Chim. Acta, 2006. 368: 33-47.

48. Kubes $\mathrm{P}$, Kerfoot SM. Leukocyte recruitment in the microcirculation: the rolling paradigm revisited. News Physiol. Sci., 2001. 16: 76-80. 
49. Stehouwer CD. Is measurement of endothelial dysfunction clinically useful? Eur. J. Clin. Invest., 1999. 29: 45961.

50. Joannides R, Haefeli WE, Linder L, Richard V, Bakkali EH, Thuillez C, Luscher TF. Nitric oxide is responsible for flow-dependent dilatation of human peripheral conduit arteries in vivo. Circulation, 1995. 91: 1314-9.

51. Vidakovic R, Schouten O, Kuiper R, Hoeks SE, Flu WJ, van Kuijk JP, Goei D, Verhagen HJ, Neskovic AN, Poldermans $\mathrm{D}$. The prevalence of polyvascular disease in patients referred for peripheral arterial disease. Eur. J. Vasc. Endovasc. Surg., 2009. 38: 435-40.

52. Paraskevas KI, Mikhailidis DP, Liapis CD. Internal carotid artery occlusion: association with atherosclerotic disease in other arterial beds and vascular risk factors. Angiology, 2007. 58: 329-35.

53. Paraskevas KI, Mikhailidis DP, Liapis CD. Severe, but not moderate, carotid atherosclerosis may predict concomitant vascular disease in other arterial beds. Stroke, 2010. 41: e597.

54. Wu YW, Lin MS, Lin YH, Chao CL, Kao HL. Prevalence of concomitant atherosclerotic arterial diseases in patients with significant cervical carotid artery stenosis in Taiwan. Int. J. Cardiovasc. Imaging, 2007. 23: 433-9.

55. Doonan AL, Karha J, Carrigan TP, Bavry AA, Begelman SM, Ellis SG, Yadav J, Bhatt DL. Presence of carotid and peripheral arterial disease in patients with left main disease. Am. J. Cardiol., 2007. 100: 1087-9.

56. Spence JD. Measurement of carotid plaque burden. JAMA neurology, 2015. 72: 383-4.

57. Fishbein MC. The vulnerable and unstable atherosclerotic plaque. Cardiovasc. Pathol., 2010. 19: 6-11.

58. Otsuka F, Joner M, Prati F, Virmani R, Narula J. Clinical classification of plaque morphology in coronary disease. Nature reviews. Cardiology, 2014. 11: 379-89.

59. Silva Marques J, Pinto FJ. The vulnerable plaque: current concepts and future perspectives on coronary morphology, composition and wall stress imaging. Rev. Port. Cardiol., 2014. 33: 101-10.

60. Ward MR, Pasterkamp G, Yeung AC, Borst C. Arterial remodeling. Mechanisms and clinical implications. Circulation, 2000. 102: 1186-91.

61. Pasterkamp G, Fitzgerald PF, de Kleijn DP. Atherosclerotic expansive remodeled plaques: a wolf in sheep's clothing. J. Vasc. Res., 2002. 39: 514-23.

62. Heusch G, Libby P, Gersh B, Yellon D, Bohm M, Lopaschuk G, Opie L. Cardiovascular remodelling in coronary artery disease and heart failure. Lancet, 2014. 383: 1933-43.

63. Ruiz JL, Hutcheson JD, Aikawa E. Cardiovascular calcification: current controversies and novel concepts. Cardiovasc. Pathol., 2015. 24: 207-212.

64. Zhang Y, Guallar E, Qiao Y, Wasserman BA. Is carotid intima-media thickness as predictive as other noninvasive techniques for the detection of coronary artery disease? Arterioscler. Thromb. Vasc. Biol., 2014. 34: $1341-5$.

65. Gepner AD, Young R, Delaney JA, Tattersall MC, Blaha MJ, Post WS, Gottesman RF, Kronmal R, Budoff MJ, Burke GL, Folsom AR, Liu K, Kaufman J, Stein JH. Comparison of coronary artery calcium presence, carotid plaque presence, and carotid intima-media thickness for cardiovascular disease prediction in the Multi-Ethnic Study of Atherosclerosis. Circ. Cardiovasc. Imaging, 2015. 8.

66. Mathiesen EB, Johnsen SH, Wilsgaard T, Bonaa KH, Lochen ML, Njolstad I. Carotid plaque area and intimamedia thickness in prediction of first-ever ischemic stroke: a 10-year follow-up of 6584 men and women: the Tromso Study. Stroke, 2011. 42: 972-8.

67. Folsom AR, Kronmal RA, Detrano RC, O'Leary DH, Bild DE, Bluemke DA, Budoff MJ, Liu K, Shea S, Szklo M, Tracy RP, Watson KE, Burke GL. Coronary artery calcification compared with carotid intima-media thickness in the prediction of cardiovascular disease incidence: the Multi-Ethnic Study of Atherosclerosis (MESA). Arch. Intern. Med., 2008. 168: 1333-9.

68. Charlesworth JA, Peake PW, Campbell LV, Pussell BA, O'Grady S, Tzilopoulos T. The influence of oral lipid loads on acylation stimulating protein (ASP) in healthy volunteers. Int. J. Obes. Relat. Metab. Disord., 1998. 22: 1096102.

69. Dejeans N, Herosimczyk A, Sayd T, Chambon C, Martin JF, Maier JA, Tauveron I, Mazur A. Effect of a high-fat challenge on the proteome of human postprandial plasma. Clin. Nutr., 2013. 32: 468-71.

70. Meijssen S, van Dijk H, Verseyden C, Erkelens DW, Cabezas MC. Delayed and exaggerated postprandial complement component 3 response in familial combined hyperlipidemia. Arterioscler. Thromb. Vasc. Biol., 2002. 22: 811-6.

71. Peake PW, Kriketos AD, Campbell LV, Charlesworth JA. Response of the alternative complement pathway to an oral fat load in first-degree relatives of subjects with type II diabetes. Int. J. Obes. (Lond), 2005. 29: 429-35.

72. Westerink J, Hajer GR, Kranendonk ME, Schipper HS, Monajemi H, Kalkhoven E, Visseren FL. An oral mixed fat load is followed by a modest anti-inflammatory adipocytokine response in overweight patients with metabolic syndrome. Lipids, 2014. 49: 247-54.

73. Halkes CJ, van Dijk H, de Jaegere PP, Plokker HW, van Der Helm Y, Erkelens DW, Castro Cabezas M. Postprandial increase of complement component 3 in normolipidemic patients with coronary artery disease: effects of expanded-dose simvastatin. Arterioscler. Thromb. Vasc. Biol., 2001. 21: 1526-30. 
74. Sodowski K, Zwirska-Korczala K, Kuka D, Kukla M, Budziszewska P, Zebaty A, Wender-Ozegowska E, Baumert $\mathrm{M}$, Wloch A. Acylation stimulating protein is associated with pregnancy weight gain. J. Physiol. Pharmacol., 2008. 59 Suppl 4: 33-43.

75. van Oostrom AJ, Alipour A, Plokker TW, Sniderman AD, Cabezas MC. The metabolic syndrome in relation to complement component 3 and postprandial lipemia in patients from an outpatient lipid clinic and healthy volunteers. Atherosclerosis, 2007. 190: 167-73.

76. Wolach B, Eliakim A, Gavrieli R, Kodesh E, Yarom Y, Schlesinger M, Falk B. Aspects of leukocyte function and the complement system following aerobic exercise in young female gymnasts. Scand. J. Med. Sci. Sports, 1998. 8: 91-7.

77. Wang JS, Chiu YT. Systemic hypoxia enhances exercise-mediated bactericidal and subsequent apoptotic responses in human neutrophils. J. Appl. Physiol., 2009. 107: 1213-22.

78. Schrauwen P, Hesselink MK, Jain M, Cianflone K. Acylation-stimulating protein: effect of acute exercise and endurance training. Int. J. Obes. (Lond), 2005. 29: 632-8.

79. Paulsen G, Benestad HB, Strom-Gundersen I, Morkrid L, Lappegard KT, Raastad T. Delayed leukocytosis and cytokine response to high-force eccentric exercise. Med. Sci. Sports Exerc., 2005. 37: 1877-83.

80. Cordova A, Sureda A, Tur JA, Pons A. Immune response to exercise in elite sportsmen during the competitive season. J. Physiol. Biochem., 2010. 66: 1-6.

81. Ihalainen J, Walker S, Paulsen G, Hakkinen K, Kraemer WJ, Hamalainen M, Vuolteenaho K, Moilanen E, Mero AA. Acute leukocyte, cytokine and adipocytokine responses to maximal and hypertrophic resistance exercise bouts. Eur. J. Appl. Physiol., 2014. 114: 2607-16.

82. Smith JD, Cianflone K, Martin J, Poirier P, Broderick TL, Noel M. Plasma adipokine and hormone changes in mountaineers on ascent to 5300 meters. Wilderness Environ. Med., 2011. 22: 107-14.

83. Burns VE, Edwards KM, Ring C, Drayson M, Carroll D. Complement cascade activation after an acute psychological stress task. Psychosom. Med., 2008. 70: 387-96.

84. Reis ES, Lange T, Kohl G, Herrmann A, Tschulakow AV, Naujoks J, Born J, Kohl J. Sleep and circadian rhythm regulate circulating complement factors and immunoregulatory properties of C5a. Brain. Behav. Immun., 2011. 25: 1416-26.

85. Hui L, Hua F, Diandong H, Hong Y. Effects of sleep and sleep deprivation on immunoglobulins and complement in humans. Brain. Behav. Immun., 2007. 21: 308-10.

86. Saleh J, Al-Khanbashi M, Al-Maarof M, Al-Lawati M, Rizvi SG, Cianflone K. Acylation-stimulating protein increases and correlates with increased progesterone levels during the luteal phase of the menstrual cycle. Eur. J. Endocrinol., 2009. 160: 301-7.

87. Mackie IJ, Campbell S, Gallimore M, Robinson G, Machin SJ. Procoagulant changes induced by oral contraceptives are balanced by an increased fibrinolytic tendency. Agents Actions. Suppl., 1992. 38 (Pt 2): 310-9.

88. Ytting H, Christensen IJ, Thiel S, Jensenius JC, Svendsen MN, Nielsen L, Lottenburger T, Nielsen HJ. Biological variation in circulating levels of mannan-binding lectin (MBL) and $\mathrm{MBL}$-associated serine protease-2 and the influence of age, gender and physical exercise. Scand. J. Immunol., 2007. 66: 458-64.

89. Saevarsdottir S, Oskarsson OO, Aspelund T, Eiriksdottir G, Vikingsdottir T, Gudnason V, Valdimarsson H. Mannan binding lectin as an adjunct to risk assessment for myocardial infarction in individuals with enhanced risk. J. Exp. Med., 2005. 201: 117-25.

90. Calder PC, Ahluwalia N, Albers R, Bosco N, Bourdet-Sicard R, Haller D, Holgate ST, Jonsson LS, Latulippe ME, Marcos A, Moreines J, M'Rini C, Muller M, Pawelec G, van Neerven RJ, Watzl B, Zhao J. A consideration of biomarkers to be used for evaluation of inflammation in human nutritional studies. Br. J. Nutr., 2013. 109 Suppl 1: S1-34.

91. McClearn GE. The reliability and stability of biomarkers of aging. Ann. N. Y. Acad. Sci., 1992. 673: 1-8.

92. Shklyar I, Pasternak A, Kapur K, Darras BT, Rutkove SB. Composite biomarkers for assessing Duchenne muscular dystrophy: an initial assessment. Pediatr. Neurol., 2015. 52: 202-5.

93. Hubel A, Spindler R, Skubitz AP. Storage of human biospecimens: selection of the optimal storage temperature. Biopreserv. Biobank., 2014. 12: 165-75.

94. Zander J, Bruegel M, Kleinhempel A, Becker S, Petros S, Kortz L, Dorow J, Kratzsch J, Baber R, Ceglarek U, Thiery J, Teupser D. Effect of biobanking conditions on short-term stability of biomarkers in human serum and plasma. Clin. Chem. Lab. Med., 2014. 52: 629-39.

95. Doumatey AP, Zhou J, Adeyemo A, Rotimi C. High sensitivity C-reactive protein (Hs-CRP) remains highly stable in long-term archived human serum. Clin. Biochem., 2014. 47: 315-8.

96. de Jager W, Bourcier K, Rijkers GT, Prakken BJ, Seyfert-Margolis V. Prerequisites for cytokine measurements in clinical trials with multiplex immunoassays. BMC immunology, 2009. 10: 52.

97. Tripepi G, Jager KJ, Dekker FW, Zoccali C. Selection bias and information bias in clinical research. Nephron Clin. Pract., 2010. 115: c94-9.

98. Dohoo IR. Bias--is it a problem, and what should we do? Prev. Vet. Med., 2014. 113: 331-7. 
99. Hartweg J, Gunter M, Perera R, Farmer A, Cull C, Schalkwijk C, Kok A, Twaalfhoven H, Holman R, Neil A. Stability of soluble adhesion molecules, selectins, and C-reactive protein at various temperatures: implications for epidemiological and large-scale clinical studies. Clin. Chem., 2007. 53: 1858-60.

100. Hill AB. The environment and disease: association or causation? Bull. World Health Organ., 2005. 83: 796-798.

101. Greenland S, Morgenstern H. Confounding in health research. Annu. Rev. Public Health, 2001. 22: 189-212.

102. Suttorp MM, Siegerink B, Jager KJ, Zoccali C, Dekker FW. Graphical presentation of confounding in directed acyclic graphs. Nephrol. Dial. Transplant., 2014.

103. Franks PW. Obesity, inflammatory markers and cardiovascular disease: distinguishing causality from confounding. J. Hum. Hypertens., 2006. 20: 837-40.

104. le Cessie S, Debeij J, Rosendaal FR, Cannegieter SC, Vandenbroucke JP. Quantification of bias in direct effects estimates due to different types of measurement error in the mediator. Epidemiology, 2012. 23: 551-60.

105. VanderWeele TJ, Valeri L, Ogburn EL. The role of measurement error and misclassification in mediation analysis: mediation and measurement error. Epidemiology, 2012. 23: 561-4.

106. Wlazlo N, van Bussel CT, Van Greevenbroek MM, Schalkwijk C, Bravenboer B, Ferreira I, Stehouwer CD, Mediation analyses in aetiological biomedical research: the influence of random error. In: Novel aspects of insulin resistance and type 2 diabetes mellitus: Iron metabolism, the complement system, and liver cirrhosis. 2014, Ede: Ponsen \& Looijen.

107. Schisterman EF, Cole SR, Platt RW. Overadjustment bias and unnecessary adjustment in epidemiologic studies. Epidemiology, 2009. 20: 488-95.

108. Rothman KJ, Greenland S, Lash TJ, Modern Epidemiology. 2008, Philadelphia: Lippincott Williams And Wilkins.

109. Greenland S, Maclure M, Schlesselman JJ, Poole C, Morgenstern H. Standardized regression coefficients: a further critique and review of some alternatives. Epidemiology, 1991. 2: 387-92.

110. Bouter LM, van Dongen MCJM, Zielhuis GA, Epidemiologisch onderzoek: opzet en interpretatie. Vol. 6. 2010, Houten: Bohn Stefleu van Loghum.

111. Engstrom G, Hedblad B, Janzon L, Lindgarde F. Complement C3 and C4 in plasma and incidence of myocardial infarction and stroke: a population-based cohort study. Eur. J. Cardiovasc. Prev. Rehabil., 2007. 14: 392-7.

112. Muscari A, Bozzoli C, Puddu GM, Sangiorgi Z, Dormi A, Rovinetti C, Descovich GC, Puddu P. Association of serum C3 levels with the risk of myocardial infarction. Am. J. Med., 1995. 98: 357-64.

113. Onat A, Hergenc G, Can G, Kaya Z, Yuksel H. Serum complement C3: a determinant of cardiometabolic risk, additive to the metabolic syndrome, in middle-aged population. Metabolism, 2010. 59: 628-34.

114. Ramadass M, Ghebrehiwet B, Kew RR. Enhanced recognition of plasma proteins in a non-native state by complement C3b. A possible clearance mechanism for damaged proteins in blood. Mol. Immunol., 2015. 64: 55-62.

115. Ramadass M, Ghebrehiwet B, Smith RJ, Kew RR. Generation of multiple fluid-phase C3b:plasma protein complexes during complement activation: possible implications in C3 glomerulopathies. J. Immunol., 2014. 192: 1220-30.

116. Luc G, Empana JP, Morange P, Juhan-Vague I, Arveiler D, Ferrieres J, Amouyel P, Evans A, Kee F, Bingham A, Machez E, Ducimetiere P. Adipocytokines and the risk of coronary heart disease in healthy middle aged men: the PRIME Study. Int. J. Obes. (Lond), 2010. 34: 118-26.

117. Prugger C, Luc G, Haas B, Arveiler D, Machez E, Ferrieres J, Ruidavets JB, Bingham A, Montaye M, Amouyel P, Yarnell J, Kee F, Ducimetiere P, Empana JP. Adipocytokines and the risk of ischemic stroke: the PRIME Study. Ann. Neurol., 2012. 71: 478-86.

118. Jawien J, Nastalek P, Korbut R. Mouse models of experimental atherosclerosis. J. Physiol. Pharmacol., 2004. 55: 503-17.

119. Bhatia VK, Yun S, Leung V, Grimsditch DC, Benson GM, Botto MB, Boyle JJ, Haskard DO. Complement C1q reduces early atherosclerosis in low-density lipoprotein receptor-deficient mice. Am. J. Pathol., 2007. 170: 416-26.

120. Lewis MJ, Malik TH, Ehrenstein MR, Boyle JJ, Botto M, Haskard DO. Immunoglobulin M is required for protection against atherosclerosis in low-density lipoprotein receptor-deficient mice. Circulation, 2009. 120: 417-26.

121. Matthijsen RA, de Winther MP, Kuipers D, van der Made I, Weber C, Herias MV, Gijbels MJ, Buurman WA. Macrophage-specific expression of mannose-binding lectin controls atherosclerosis in low-density lipoprotein receptor-deficient mice. Circulation, 2009. 119: 2188-95.

122. Hillian AD, McMullen MR, Sebastian BM, Roychowdhury S, Kashyap SR, Schauer PR, Kirwan JP, Feldstein AE, Nagy LE. Mice lacking C1q are protected from high fat diet-induced hepatic insulin resistance and impaired glucose homeostasis. J. Biol. Chem., 2013. 288: 22565-75.

123. Busche MN, Walsh MC, McMullen ME, Guikema BJ, Stahl GL. Mannose-binding lectin plays a critical role in myocardial ischaemia and reperfusion injury in a mouse model of diabetes. Diabetologia, 2008. 51: 1544-51. 
124. Pavlov VI, La Bonte LR, Baldwin WM, Markiewski MM, Lambris JD, Stahl GL. Absence of mannose-binding lectin prevents hyperglycemic cardiovascular complications. Am. J. Pathol., 2012. 180: 104-12.

125. Malik TH, Cortini A, Carassiti D, Boyle JJ, Haskard DO, Botto M. The alternative pathway is critical for pathogenic complement activation in endotoxin- and diet-induced atherosclerosis in low-density lipoprotein receptor-deficient mice. Circulation, 2010. 122: 1948-56.

126. Persson L, Boren J, Robertson AK, Wallenius V, Hansson GK, Pekna M. Lack of complement factor C3, but not factor B, increases hyperlipidemia and atherosclerosis in apolipoprotein $\mathrm{E}^{-/}$low-density lipoprotein receptor ${ }^{-1}$ mice. Arterioscler. Thromb. Vasc. Biol., 2004. 24: 1062-7.

127. Steiner T, Francescut L, Byrne S, Hughes T, Jayanthi A, Guschina I, Harwood J, Cianflone K, Stover C, Francis S. Protective role for properdin in progression of experimental murine atherosclerosis. PLoS ONE, 2014. 9: e92404.

128. Morigi M, Galbusera M, Gastoldi S, Locatelli M, Buelli S, Pezzotta A, Pagani C, Noris M, Gobbi M, Stravalaci M, Rottoli D, Tedesco F, Remuzzi G, Zoja C. Alternative pathway activation of complement by Shiga toxin promotes exuberant C3a formation that triggers microvascular thrombosis. J. Immunol., 2011. 187: 172-80.

129. Turner NA, Moake J. Assembly and activation of alternative complement components on endothelial cellanchored ultra-large von Willebrand factor links complement and hemostasis-thrombosis. PLoS ONE, 2013. 8: e59372.

130. Saggu G, Cortes C, Emch HN, Ramirez G, Worth RG, Ferreira VP. Identification of a novel mode of complement activation on stimulated platelets mediated by properdin and C3(H2O). J. Immunol., 2013. 190: 6457-67.

131. Conway EM. Reincarnation of ancient links between coagulation and complement. J. Thromb. Haemost., 2015. 13 Suppl 1: S121-32.

132. Oikonomopoulou K, Ricklin D, Ward PA, Lambris JD. Interactions between coagulation and complement--their role in inflammation. Semin. Immunopathol., 2012. 34: 151-65.

133. Markiewski MM, Nilsson B, Ekdahl KN, Mollnes TE, Lambris JD. Complement and coagulation: strangers or partners in crime? Trends Immunol., 2007. 28: 184-92.

134. Peerschke El, Yin W, Ghebrehiwet B. Platelet mediated complement activation. Adv. Exp. Med. Biol., 2008. 632: 81-91.

135. Hugli TE. Structure and function of the anaphylatoxins. Springer Semin. Immunopathol., 1984. 7: 193-219.

136. Guo RF, Ward PA. Role of C5a in inflammatory responses. Annu. Rev. Immunol., 2005. 23: 821-52.

137. Manthey HD, Woodruff TM, Taylor SM, Monk PN. Complement component 5a (C5a). Int. J. Biochem. Cell Biol., 2009. 41: 2114-7.

138. Lewis RD, Jackson CL, Morgan BP, Hughes TR. The membrane attack complex of complement drives the progression of atherosclerosis in apolipoprotein E knockout mice. Mol. Immunol., 2010. 47: 1098-105.

139. An G, Miwa T, Song WL, Lawson JA, Rader DJ, Zhang Y, Song WC. CD59 but not DAF deficiency accelerates atherosclerosis in female ApoE knockout mice. Mol. Immunol., 2009. 46: 1702-9.

140. Wu G, Hu W, Shahsafaei A, Song W, Dobarro M, Sukhova GK, Bronson RR, Shi GP, Rother RP, Halperin JA, Qin $X$. Complement regulator CD59 protects against atherosclerosis by restricting the formation of complement membrane attack complex. Circ. Res., 2009. 104: 550-8.

141. Yun S, Leung VW, Botto M, Boyle JJ, Haskard DO. Brief report: accelerated atherosclerosis in low-density lipoprotein receptor-deficient mice lacking the membrane-bound complement regulator CD59. Arterioscler. Thromb. Vasc. Biol., 2008. 28: 1714-6.

142. Manthey HD, Thomas AC, Shiels IA, Zernecke A, Woodruff TM, Rolfe B, Taylor SM. Complement C5a inhibition reduces atherosclerosis in ApoE- mice. FASEB J., 2011. 25: 2447-55.

143. Patel S, Thelander EM, Hernandez M, Montenegro J, Hassing H, Burton C, Mundt S, Hermanowski-Vosatka A, Wright SD, Chao YS, Detmers PA. ApoE $\left({ }^{-1}\right)$ mice develop atherosclerosis in the absence of complement component C5. Biochem. Biophys. Res. Commun., 2001. 286: 164-70.

144. Yang X, Peterson L, Thieringer R, Deignan JL, Wang X, Zhu J, Wang S, Zhong H, Stepaniants S, Beaulaurier J, Wang IM, Rosa R, Cumiskey AM, Luo JM, Luo Q, Shah K, Xiao J, Nickle D, Plump A, Schadt EE, Lusis AJ, Lum PY. Identification and validation of genes affecting aortic lesions in mice. J. Clin. Invest., 2010. 120: 2414-22.

145. Buono C, Come CE, Witztum JL, Maguire GF, Connelly PW, Carroll M, Lichtman AH. Influence of C3 deficiency on atherosclerosis. Circulation, 2002. 105: 3025-31.

146. Gushiken FC, Han H, Li J, Rumbaut RE, Afshar-Kharghan V. Abnormal platelet function in C3-deficient mice. J. Thromb. Haemost., 2009. 7: 865-70.

147. Turner MW. Mannose-binding lectin: the pluripotent molecule of the innate immune system. Immunol. Today, 1996. 17: 532-40.

148. Ivanova M, Ruiqing J, Matsushita M, Ogawa T, Kawai S, Ochiai N, Shivarov V, Maruya E, Saji H. MBL2 single nucleotide polymorphism diversity among four ethnic groups as revealed by a bead-based liquid array profiling. Hum. Immunol., 2008. 69: 877-84. 
149. Kilpatrick DC. Mannan-binding lectin: clinical significance and applications. Biochim. Biophys. Acta, 2002. 1572: 401-13.

150. Erqou S, Kip KE, Mulukutla SR, Aiyer AN, Reis SE. Racial differences in the burden of coronary artery calcium and carotid intima media thickness between Blacks and Whites. Neth. Heart J., 2015. 23: 44-51.

151. Jones MR, Diez-Roux AV, O'Neill MS, Guallar E, Sharrett AR, Post W, Kaufman JD, Navas-Acien A. Ambient air pollution and racial/ethnic differences in carotid intima-media thickness in the Multi-Ethnic Study of Atherosclerosis (MESA). J. Epidemiol. Community Health, 2015. 69: 1191-8.

152. Ricklin D, Lambris JD. Complement-targeted therapeutics. Nat. Biotechnol., 2007. 25: 1265-75.

153. Ricklin D, Lambris JD. Therapeutic control of complement activation at the level of the central component C3. Immunobiology, 2015 [doi: 10.1016/j.imbio.2015.06.012].

154. Brai M, Accardo P, Bellavia D. Polymorphism of the complement components in human pathology. Ann. Ital. Med. Int., 1994. 9: 167-72.

155. Kraivong R, Vasanawathana S, Limpitikul W, Malasit P, Tangthawornchaikul N, Botto M, Screaton GR, Mongkolsapaya J, Pickering MC. Complement alternative pathway genetic variation and Dengue infection in the Thai population. Clin. Exp. Immunol., 2013. 174: 326-34.

156. Heurich M, Martinez-Barricarte R, Francis NJ, Roberts DL, Rodriguez de Cordoba S, Morgan BP, Harris CL. Common polymorphisms in C3, factor B, and factor $\mathrm{H}$ collaborate to determine systemic complement activity and disease risk. Proc. Natl. Acad. Sci. U. S. A., 2011. 108: 8761-6.

157. Harris $\mathrm{CL}$, Heurich M, Rodriguez de Cordoba S, Morgan BP. The complotype: dictating risk for inflammation and infection. Trends Immunol., 2012. 33: 513-21.

158. Zhu ZB, Totemchokchyakarn K, Atkinson TP, Volanakis JE. Molecular defects leading to human complement component C6 deficiency in an African-American family. Clin. Exp. Immunol., 1998. 111: 91-6.

159. Wuillemin WA, Spath PJ, Uring-Lambert B, Straub PW. Clinical manifestations in humans of combined C7 and C4 deficiency associated with low levels of C2, C8, and C9. Complement Inflamm., 1991. 8: 70-9.

160. Westra D, Kurvers RA, van den Heuvel LP, Wurzner R, Hoppenreijs EP, van der Flier M, van de Kar NC, Warris A. Compound heterozygous mutations in the $\mathrm{C} 6$ gene of a child with recurrent infections. Mol. Immunol., 2014. 58: 201-5.

161. Thomas AD, Orren A, Connaughton J, Feighery C, Morgan BP, Roberts AG. Characterization of a large genomic deletion in four Irish families with C7 deficiency. Mol. Immunol., 2012. 50: 57-9.

162. Sjoholm AG. Inherited complement deficiency states: implications for immunity and immunological disease. APMIS, 1990. 98: 861-74.

163. Schejbel L, Fadnes D, Permin H, Lappegard KT, Garred P, Mollnes TE. Primary complement C5 deficiencies molecular characterization and clinical review of two families. Immunobiology, 2013. 218: 1304-10.

164. Ki CS, Kim JW, Kim HJ, Choi SM, Ha GY, Kang HJ, Kim WD. Two novel mutations in the C7 gene in a Korean patient with complement C7 deficiency. J. Korean Med. Sci., 2005. 20: 220-4.

165. Fukumori Y, Horiuchi T. Terminal complement component deficiencies in Japan. Exp. Clin. Immunogenet., 1998. 15: 244-8.

166. Fijen CA, Kuijper EJ, Te Bulte M, van de Heuvel MM, Holdrinet AC, Sim RB, Daha MR, Dankert J. Heterozygous and homozygous factor $\mathrm{H}$ deficiency states in a Dutch family. Clin. Exp. Immunol., 1996. 105: 511-6.

167. Falcao DA, Reis ES, Paixao-Cavalcante D, Amano MT, Delcolli MI, Florido MP, Albuquerque JA, MoraesVasconcelos D, Duarte AJ, Grumach AS, Isaac L. Deficiency of the human complement regulatory protein factor $\mathrm{H}$ associated with low levels of component C9. Scand. J. Immunol., 2008. 68: 445-55.

168. Corvini M, Randolph C, Aronin SI. Complement C7 deficiency presenting as recurrent aseptic meningitis. Ann. Allergy. Asthma. Immunol., 2004. 93: 200-5.

169. Chiang YC, Shyur SD, Huang LH, Wen TC, Lin MT, Yang HC, Liang PH. Deficiency of the seventh component of complement in a Taiwanese boy. J. Formos. Med. Assoc., 2006. 105: 770-4. 
Nederlandstalige samenvatting uGdGNgndzfg!! 


\section{Nederlandstalige samenvatting}

Hart- en vaatziekten zijn de belangrijkste doodsoorzak wereldwijd. Hart- en vaat-ziekten is een verzamelbegrip voor aandoeningen van de bloedvaten, bijvoorbeeld in het hart, de hersenen of in de benen. Aandoeningen van de bloedvaten kunnen leiden tot een hartinfarct, beroerte of perifeer arterieel vaatlijden, ook wel etalagebenen genoemd. Deze ziekten vormen een grote last zowel voor patienten als voor de gezondheidszorg. Daarom is het belangrijk om meer inzicht te krijgen in de oorzaken van hart- en vaatziekten. Het ontdekken van oorzaken voor ziekten kan bijdragen aan het ontwikkelen van nieuwe behandelmethoden. Reeds bekende oorzaken van hart- en vaatziekten zijn laaggradige ontsteking, ongezonde en slecht functionerende vaten (vaatdisfunctie) en slagaderverkalking (atherosclerose). Leefstijlfactoren zoals overgewicht en roken kunnen leiden tot laaggradige ontsteking van de bloedvaten. In de loop van de tijd leidt laaggradige ontsteking tot vaatdisfunctie. Door een verslechterde functie van de binnenbekleding van de bloedvaten (het endotheel) ontstaat bijvoorbeeld endotheeldisfunctie. Een van de gevolgen van endotheeldisfunctie is slagaderverkalking, de verharding en vernauwing van de vaatwanden. Vernauwing van de slagaders zorgt voor een verslechterde bloed- en zuurstofvoorziening en kan leiden tot de afsluiting of scheuring van vaten. Door afgesloten vaten kan bloed minder goed aan- en afgevoerd worden en functioneren de weefsels minder goed, zoals bij etalagebenen, of kunnen deels afsterven, zoals bij een hartinfarct of beroerte.

In de laatste jaren is er bijzondere belangsteling voor de rol van het afweersysteem gekomen in het ontstaan van hart- en vaatziekten. Het afweersysteem zorgt niet alleen voor de afweer tegen indringers, maar speelt ook een belangrijke rol in het initiëren van onsteking en het aansturen van weefselvernieuwing en -herstel. Het afweersysteem is ook betrokken bij het onderhoud van de bloedvaten, en onderzoek laat zien dat het afweersysteem een rol speelt in ontsteking van de bloedvaten, vaatdisfunctie en slagaderverkalking.

Een belangrijk onderdeel van het afweersysteem is het complementsysteem. Het complementsysteem bestaat uit verschillende eiwitten, de zogenaamde complementfactoren. Complementfactoren zijn aanwezig in het bloed en in de weefsels en kunnen via kettingreacties aanleiding geven tot productie van lokstoffen en signaalstoffen en kunnen bijdragen aan het opruimen van beschadigde weefsels. Een kettingreactie kan bijvoorbeeld gestart worden door het herkennen van beschadigde weefsels. Die kettingreacties van complementactivatie kunnen verlopen via drie verschillende routes: de klassieke route, de lectineroute en de alternatieve route. Alle drie deze routes maken eigen signaalstoffen vrij en ze monden uit op een gezamenlijke eindroute. Het voortschrijden van de kettingreactie tot de gezamenlijke eindroute leidt onder meer tot de productie van krachtige complementsignaalstoffen. Complementsignaalstoffen veroorzaken ontsteking, daarom kan overmatige activatie van het complementsysteem aanzienlijke schade toebrengen aan de weefsels.

In dit proefschrift onderzochten we de rol van het complementsysteem in het ontstaan van hart- en vaatziekten. Daarbij hebben we gebruik gemaakt van de CODAM studie. In het bloed van deelnemers van de CODAM studie hebben we de hoeveelheiden van verschillende complementfactoren gemeten, en gekeken of deze samenhingen met laaggradige 
ontsteking, endotheeldisfunctie, slagaderverkalking en het krijgen van hart- en vaatziekten. De belangrijkste bevindingen zijn hieronder beschreven:

\section{Factoren van de alternatieve route bevorderen het onstaan van hart- en vaatziekten}

De kettingreactie van de alternatieve route kan spontaan en door toeval gestart worden. Eerder onderzoek in mensen heeft laten zien dat spontane sterke en ongerichte activatie van de alternatieve route tot schade aan het oog kan leiden. Dit suggereert dat een grotere hoeveelheid alternatieve complementfactoren in het bloed kan leiden tot meer spontane en ongerichte complementactivatie in de bloedvaten, met als gevolg beschadiging van de vaten. In dit proefschrift hebben we drie complementfactoren van de alternatieve route gemeten: twee factoren die deel vormen van de kettingreactie, en één factor die als aanjager van de kettingreactie werkt. Grotere hoeveelheiden van alle drie factoren van de alternatieve route hingen samen met meer ontsteking en meer endotheeldisfunctie. Daarnaast gaf een grotere hoeveelheid van de aanjager een hoger risico op het krijgen van hart- en vaatziekten. Dit suggereert dat het spontane starten van de alternatieve route inderdaad bij kan dragen aan vaatschade, en dat in het bijzondere het aanjagen van de kettingreactie het onstaan van harten vaatziekten bevordert.

\section{Factoren van de klassieke route en de lectineroute hebben zowel beschermende als beschadigende effekten op het hart- en vaatstelsel}

De kettingreacties van de klassieke route en de lectineroutes worden beide gestart door het herkennen van gevaarsignalen, zoals bijvoorbeeld afvalproducten of beschadigde weefsels. Uit onderzoek in proefdieren is gebleken, dat het starten van kettingreacties via de klassieke route en de lectineroute beschermend kan zijn tegen slagaderverkalking. Een mogelijke verklaring hiervoor is dat de klassieke route en de lectineroute belangrijk zijn voor het opruimen van afvalproducten en dat minder afvalproducten leiden tot minder hart- en vaatziekten. In dit proefschrift hebben we factoren van de klassieke route en de lectineroute gemeten die gevaarsignalen kunnen herkennen. Het bleek dat zowel grote als ook kleine hoeveelheiden van deze herkenningsfactoren samenhingen met meer ontsteking of het onstaan van hart- en vaatziekten. Dit suggereert een tweeledige rol van de klassieke route en de lectineroute in mensen: Aan de ene kant bestaan er beschermende effecten op het vaatstelsel. Daardoor kan een tekort aan herkenningsfactoren leiden tot onvoldoende opruiming van afvalproducten. Dit kan het onstaan van hart- en vaatziekten bevorderen. Aan de andere kant veroorzaakt een teveel aan herkenningsfactoren van de klassieke route en de lectineroute overmatige complementactivatie. Dit kan bijdragen aan ontsteking en vaatschade. 
De meest sterke complementsignaalstoffen hangen niet samen met hart- en vaatziekten

Vanuit de gezamelijke eindroute kunnen complementsignaalstoffen vrijgemaakt worden. Het is bekend dat deze complementsignaalstoffen verschillen in de mate waarin ze ontsteking kunnen aanjagen. Eerder onderzoek in proefdieren liet zien, dat al deze complementsignaalstoffen de ontwikkeling van slagaderverkalking bevorderen. In onze studies hing een signaalstof van gematigde sterkte samen met slagaderverkalking. Echter hingen de twee sterkste complementsignaalstoffen niet samen met slagaderverkalking of hart- en vaatziekten. Dit suggereert dat de sterkte van complementsignaalstoffen niet bepalend is voor de mate waarin ze samenhangen met hart- en vaatziekten. Een mogelijke verklaring hiervoor is dat complementsignaalstoffen voornamelijk binnen weefsels vrijgemaakt worden. Mogelijk is de hoeveelheid in het bloed geen heel goede weerspiegeling van de hoeveelheid complementsignaalstoffen die binnen de weefsels geproduceerd is.

\section{Conclusie}

Dit proefschrift laat zien dat complementfactoren in het bloed samenhangen met de ontwikkeling van hart- en vaatziekten. In het bijzonder leidt spontane en ongerichte complementactivatie via de alternatieve route tot ontsteking, endotheeldisfunctie en het ontstaan van hart- en vaatziekten. Daarentegen leiden zowel een teveel als een tekort van herkenningsfactoren van de klassieke route en de lectineroute tot ontsteking en het ontstaan van hart- en vaatziekten. Echter is mogelijk het meten van de sterkste complementsignaalstoffen in het bloed geen goede weerspiegeling van de bijdrage van complementactivatie aan hart- en vaatziekten.

Deze bevindingen geven inzicht in het ontstaan van hart- en vaatziekten in mensen en kunnen bijdragen aan het ontwikkelen van nieuwe preventie- en behandelmethoden. Complementfactoren van de alternatieve route zijn mogelijk geschikt voor het voorspellen van de kans op het krijgen van hart- en vaatziekten. Dat vergroot de mogelijkheid om mensen met een verhoogd risico te herkennen en vroeger en intensiever te behandelen. Daarnaast zijn deze bevindingen bemoedigend voor vervolgonderzoek, om methoden te ontwikkelen die leiden tot de remming van spontane complementactivatie via de alternatieve route. 


\section{Valorisation addendum $\wedge$ glomiggfion gddGndrim}




\section{Valorisation addendum}

Valorisation is the "process of value-creation out of knowledge, by making this knowledge suitable and available for economic or societal utilisation" [1]. Economic utilisation involves for example commercialisation of knowledge by generating patents and license contracts [2]. Societal utilisation means making knowledge available to individuals and public organizations who might apply research findings.

The studies included in the current thesis investigated the associations between plasma complement factors and development of cardiovascular disease (CVD) in a cohort of middleaged to elderly individuals. We showed that circulating complement factors are associated with CVD and processes contributing to CVD. These findings are relevant to healthcare providers and to individuals at risk for CVD. However, this research project did not explore hypotheses that could directly lead to patent applications. Hence, this chapter describes how the current findings may be applied in order to decrease cardiovascular burden and provides an outlook on the steps to be taken in order to translate the current findings into prevention and treatment strategies of CVD.

The investigation of novel risk factors for CVD can create value at different levels. First, it can result in finding novel risk markers in order to assess the cardiovascular risk of an individual. Quantifying an individual's cardiovascular risk can be utilized in clinical decision making, and can decrease cardiovascular burden by delivering medical interventions earlier and by providing personalized treatment. Identification of novel risk markers can also yield economic utilization, for example when the measurement method can be patented and is applicable on a large scale in clinical practice. Second, novel risk factors for CVD may pose potential targets for novel medical treatments or healthy behaviours and thereby decrease overall CVD risk at the population level. Third, elucidating the pathophysiology of CVD creates valuable knowledge by itself as it advances the understanding of a highly prevalent and harmful disease and provides the basis for further investigations.

\section{Complement factors as potential novel risk markers}

Our investigations suggested that properdin and $\mathrm{C} 1 \mathrm{q}$ may potentially be suitable as risk markers of CVD. Plasma properdin and C1q provided independent information on cardiovascular risk, while other complement factors were associated with early processes of vascular damage but not with future disease. The American Heart Association recommends several steps in the identification of novel markers of cardiovascular risk [3]. Accordingly, an "initial proof of concept", and subsequently a "prospective validation in independent populations" are the first steps to identify risk markers of CVD. The data on properdin can be considered as first proof of concept, because this is the first investigation of properdin in human CVD. Regarding C1q, our study provided the first prospective evaluation, as previous studies on C1q in human CVD were cross-sectional. This thesis thus provides the first steps in the investigation of properdin and C1q as potential novel markers of CVD risk. To confirm their suitability as risk markers, replication of our findings in prospective evaluations in other, larger populations is needed for both properdin and C1q. Importantly, in order to be used 
for risk prediction in practice, the laboratory measurements of risk markers must be reliable, accurate and cost-effective [4]. In the present investigation, the laboratory measurement of C1q was only moderately accurate (inter-assay coefficient of variation 18.5\%), and for properdin, two-to-three-fold higher concentrations were reported in previous studies $[5,6]$. Thereby our study also points up the need to optimize and standardize the laboratory methods prior to a potential routine use in the clinic.

\section{Complement activation pathways as potential targets}

Factors that are associated with CVD and processes contributing to CVD may serve as potential targets of future treatment and prevention strategies, provided that they also play a causal role in CVD. The current findings suggest a role for all complement activation pathways in CVD, as factors from each pathway were associated with pathophysiological processes, i.e. inflammation, endothelial dysfunction and/or atherosclerosis. Notably, our investigations suggested that the alternative pathway may be most relevant in development of CVD. Therefore, the alternative pathway may be the most promising target for potential treatment and prevention strategies. First, relevance of the alternative pathway should be confirmed at the tissue-level, given that our findings are based on observations on systemic complement concentrations. If confirmed, the alternative pathway may be a particularly interesting target because it can be activated spontaneously. Understanding in which circumstances the alternative pathway is activated could result in novel treatment strategies and optimization of current treatment approaches. For example, current strategies to develop antioxidant therapies for CVD could take potential effects on alternative pathway activation into account, as the alternative pathway can be activated by oxidative stress $[7,8]$. Furthermore, alternative pathway activation may be partly modifiable by lifestyle factors, as lipid-rich meals and smoking were reported to affect the alternative pathway [9-11]. A better understanding of the interplay between lifestyle and alternative pathway activation may provide further rationale to support the promotion of healthy behaviours at the population level.

\section{Novel insights into the pathophysiology of CVD}

This comprehensive evaluation of complement factors provided several novel insights that enrich the understanding of CVD. A better understanding of the complexity of CVD is valuable by itself and, in addition, provides the basis for further research. For example, our studies suggested that MASP-3 and MAp44 may play a role in endothelial dysfunction independently of lectin pathway activation. Although this finding cannot directly be translated into an application to recognize or intervene on cardiovascular risk, it may stimulate further research and potentially result in the discovery of novel pathways in endothelial (dys)function. Endothelial dysfunction is not only involved in CVD, but also in development of other chronic diseases such as type 2 diabetes mellitus $[12,13]$. Identification of novel pathophysiological interplays in endothelial dysfunction may in the long-term perspective yield novel targets to decrease chronic diseases such as CVD or type 2 diabetes. 
In conclusion, there is a clear potential to apply the current findings in the development of clinical risk markers and in the development of treatment and prevention strategies. For this, confirmation of our findings in other studies and optimisation of the laboratory measurements are needed in advance. Furthermore, it should be kept in mind that treatment and prevention strategies must be very cost-effective in order to be applicable on a large scale. Screening of individuals based on risk markers, personalized interventions and (development of) novel pharmacological treatments are highly costly. Viewed realistically, societal utilization of the present findings may thus be feasible in the more distant future, and potentially only in high-income countries. Nevertheless, each and every small contribution to advance the understanding of CVD is one step forward to combat a major health issue. 


\section{References}

1. Netherlands Proteomic Centre. What is valorisation? [cited 11 December 2015]; retrieved from http://www.netherlandsproteomicscentre.nl/npc/valorisation/what-is-valorisation.

2. De Jonge B, Louwaars N. Valorizing science: whose values? Science \& society series on convergence research. EMBO Rep., 2009. 10: 535-9.

3. Hlatky MA, Greenland P, Arnett DK, Ballantyne CM, Criqui MH, Elkind MS, Go AS, Harrell FE, Jr., Hong Y, Howard BV, Howard VJ, Hsue PY, Kramer CM, McConnell JP, Normand SL, O'Donnell CJ, Smith SC, Jr., Wilson PW. Criteria for evaluation of novel markers of cardiovascular risk: a scientific statement from the American Heart Association. Circulation, 2009. 119: 2408-16.

4. Stampfer MJ, Ridker PM, Dzau VJ. Risk factor criteria. Circulation, 2004. 109: IV3-5.

5. Schwaeble WJ, Reid KB. Does properdin crosslink the cellular and the humoral immune response? Immunol. Today, 1999. 20: 17-21.

6. Somani R, Richardson VR, Standeven KF, Grant PJ, Carter AM. Elevated properdin and enhanced complement activation in first-degree relatives of South Asian subjects with type 2 diabetes. Diabetes Care, 2012. 35: 894-99.

7. Spychalowicz A, Wilk G, Sliwa T, Ludew D, Guzik TJ. Novel therapeutic approaches in limiting oxidative stress and inflammation. Curr. Pharm. Biotechnol., 2012. 13: 2456-66.

8. Pujol-Lereis LM, Schafer N, Kuhn LB, Rohrer B, Pauly D. Interrelation Between Oxidative Stress and Complement Activation in Models of Age-Related Macular Degeneration. Adv. Exp. Med. Biol., 2016. 854: 8793.

9. Peake PW, Kriketos AD, Campbell LV, Charlesworth JA. Response of the alternative complement pathway to an oral fat load in first-degree relatives of subjects with type II diabetes. Int. J. Obes. (Lond), 2005. 29: 429-35.

10. Westerink J, Hajer GR, Kranendonk ME, Schipper HS, Monajemi H, Kalkhoven E, Visseren FL. An oral mixed fat load is followed by a modest anti-inflammatory adipocytokine response in overweight patients with metabolic syndrome. Lipids, 2014. 49: 247-54.

11. Kew RR, Ghebrehiwet B, Janoff A. Cigarette smoke can activate the alternative pathway of complement in vitro by modifying the third component of complement. J. Clin. Invest., 1985. 75: 1000-7.

12. Del Turco S, Gaggini M, Daniele G, Basta G, Folli F, Sicari R, Gastaldelli A. Insulin resistance and endothelial dysfunction: a mutual relationship in cardiometabolic risk. Curr. Pharm. Des., 2013. 19: 2420-31.

13. Muris DM, Houben AJ, Schram MT, Stehouwer CD. Microvascular dysfunction: an emerging pathway in the pathogenesis of obesity-related insulin resistance. Rev. Endocr. Metab. Disord., 2013. 14: 29-38. 


\section{Dankwoord DsnkMOORd}

Acknowledgement $\forall C K N O M I G d$ a GWGNf

Danksagung 


\section{Dankwoord Acknowledgement Danksagung}

Conducting the research presented in this thesis substantially advanced my scientific but also my professional and personal development. I am deeply grateful for this experience and want to thank many people for their support and guidance.

Mijn promotie team, prof. dr. Coen Stehouwer, dr. Marleen van Greevenbroek en prof. dr. Ilja Arts. Ik wil jullie bedanken voor het mogelijk maken van dit onderzoek en ben jullie in het bijzonder dankbaar voor de kansen die ik naast het uitvoeren van onderzoek heb gekregen. Ik heb de M.Sc. Epidemiologie en diverse cursussen mogen volgen, en heb talrijke congressen bezocht. Verder wil ik jullie bedanken voor jullie betrokkenheid bij het zoeken naar een baan naar de promotie. Beste Coen, je bent super inspirerend als promotor en bent in veel opzichten een onbereikbaar voorbeeld. Ik ben vol bewondering voor je streven naar wetenschappelijke integriteit, je ongelofelijk efficiënte werkwijze en je belangstelling voor de kunst van de retoriek. Ook je directe en heldere communicatie vond ik heel prettig, hoewel ik soms mijn collega's voor de interpretatie van afkortingen in je e-mails moest benaderen. Beste Marleen en Ilja, ik heb door onze overleggen ongelofelijk veel geleerd. Ook wil ik jullie bedanken voor het spoedig en grondig nakijken van manuscripten en dergelijken. Marleen, je deur stond altijd open en je hebt steeds de tijd voor mij genomen, ongeacht hoe druk je het had. Toen we samen op congres waren, was het altijd gezellig. Ilja, je frisse blik op de dingen was heel nuttig en heeft deels pragmatische oplossingen opgeleverd.

Prof. dr. Casper Schalkwijk. Beste Casper, ik heb heel erg genoten van de ontspannen sfeer op onze afdeling, toe te schrijven aan jouw manier van leiden. Hoewel ik niet jouw promovendus was, was je altijd geïnteresseerd in mijn project. Daarnast wil ik je bedanken voor je hartelijke gastvrijheid - hier ook mijn dank en bewondering aan Hermine.

Dr. Boy Houben. Beste Boy, bedankt voor je gezelligheid! Voor mij waren de stapavonden tijdens congressen echte hoogtepunten - en doordat je meestal mee van de partij was heb ik me achteraf nooit schuldig van een kater gevoeld.

Dr. Carla van der Kallen. Beste Carla, bedankt voor de prettige samenwerking en je openheid tijdens congressen en bijeenkomsten.

Alle leden van de beoordelingscommissie, prof. dr. Hugo ten Cate, prof. dr. Nikolaus Marx, prof. dr. Martin Prins, dr. Judith Sluimer en dr. Leendert Trouw, wil ik graag bedanken voor het lezen en beoordelen van mijn proefschrift.

Mijn paranimf, beste Marja, wat had ik een geluk dat je toen je WESP over C3a hebt gedaan! Je bent een belangrijke vriendin geworden, en met jou heb ik mijn leukste avonden in 
Maastricht gehad. Ik wil je bedanken voor al die wandelingen, shoppingtours, etentjes, wijntjes, biertjes en überhaupt voor de gezelligheid! Ik ben super blij en dankbaar dat je op deze bijzondere dag naast me staat. Ik hoop dat we in de toekomst desondanks de afstand nog vaak afspreken, en bedank me - deels vooruit - voor je gastvrijheid.

Mijn paranimf, beste Jean, het is gewoon geweldig hoe je je voor anderen en de afdeling inzet. Je hulp bij computerproblemen is onmisbar, en je wetenschappelijke nieuwsgierigheid maakt de overleggen leuk interactief. Je staat altijd open voor groepsactiviteiten en nieuwe ideën, en je enthusiasme en dadendrang zijn super aanstekelijk. Ik ben je heel dankbaar voor het professionele design van mijn omslag. Bovendien heb je de leukste foto's ooit van mijn genomen. Ik ben super blij dat je mijn paranimf bent - bedankt Jean!

Mijn kamergenoten Lian en Marcelle. Lieve meisjes, wat was het gezellig met jullie! Eerlijk, zonder ons geklets, gebabbel, gezwets en niet te vergeten de wetenschappelijke discussies was ik de PhD minder goed doorgekomen. Jullie waren essentieel voor mijn promotie.

Mijn niet minder gezellige kamergenoten in het eerste jaar, beste Bas, Yvo en Petra, bedankt voor jullie hulp en enthousiasme. Bas en Yvo, ik wens jullie heel veel succes met jullie carrières in de kliniek als artsen en wetenschappers, maar dat zal ongetwijfeld goed komen. Petra, het is super leuk hoe open en vrolijk je bent!

Nog meer collega's van interne geneeskunde: Adriaan, Annemieke, Ben, Dennis, Dionne, Hanneke, Johanna, Jose, Katrien, Kristiaan, Maria, Marjo, Margee, Marnix, Matthias, Mitchell, Monica, Nick, Nordin, Olaf, Pauline, Remy, Roel, Stefan, Stijn, Suzan, Teba, Thomas, Vicky, bedankt voor de gezellige congressen, (lab-)uitjes, (kerst-)diners, etentjes, stapavonden, carnavalsfeesten en niet te vergeten - voor al die traktaties! Altijd weer de heerlijke Limburgse vlaai...die mis ik nu al. Ik wens jullie allen van harte succes met jullie persoonlijke en wetenschappelijke projecten. Vicky, Marjo en Margee, bedankt voor jullie geduld en praktische hulp in het lab en voor de verschillende metingen die jullie voor me hebben uitgevoerd. Stefan, in je WESP heb je de basis voor het C3a stuk gelegd, daardoor kwam ik in de luxe situatie dat ik bij het leren van SPSS een voorbeeld had. Super leuk dat we de Cambridge cursus samen gedaan hebben! Nick, bedankt voor je glasheldere uitleggen over GEE en andere statistische fenomenen, en voor je passie voor de feestmuziek. Nordin, je kwam altijd met interessante onderwerpen, die gelukkig veel mogelijkheden om te discussiëren boden - bedankt voor de discussies. Dennis, bedankt voor de lol en het luisteren. Olaf, door jij was ik zelf een keer uit in Heerlen, bedankt voor je gezelligheid. Stijn, bedankt voor een heerlijk decadente avond in Barcelona. Mitchell, bedankt voor je wetenschappelijke passie en kritisch vermogen in de werkoverleggen. Pauline, bedankt voor je optimisme en je steeds aanstekelijke goede zin. Katrien, Dionne en Monica, bedankt voor jullie goede humeur. Matthias, je kwam helaas pas laat bij onze groep, maar toch wil ik je bedanken voor je motiverende woorden in mijn laatste, drukke fase. 
I would like to thank all co-authors and collaborators who contributed to the present manuscripts with constructive comments and provision of laboratory data. Prof. dr. Edith Feskens, dr. Eugene Jansen, dr. Steffen Thiel and dr. Ingeborg Hoffmann-Petersen, it was a great pleasure to work with you.

Dit hele proefschrift baseerd op data van de CODAM studie. Ik wil alle deelnemers en onderzoekers bedanken die dit mooie data set mogelijk gemaakt hebben.

Ik ben dankbaar aan de Nederlandse hartstichting. Misschien ongebruikelijk, maar ik wil hier ook mijn dank uitspreken aan CARIM en de Universiteit Maastricht. Voor mij was het brede leeraanbod (vooral het niet wetenschappelijke leeraanbod) geen vanzelfsprekendheid, en ik heb tijdens mijn PhD erg geprofiteerd van allerlei cursussen.

Mijn vriendinnen die ik in Nederland gevonden heb, bedankt voor de leuke afwisseling en jullie betrokkenheid. Linda, bedankt voor de goede gesprekken en etentjes, je had altijd een luisterend oor. Anja en Bianca, we hebben een vergelijkbare weg genomen, maar ik had het geluk dat jullie deze weg al voor me zijn gegaan. In de afrondingsfase waren jullie mijn voorbeelden, en jullie tips en motivatie waren onmisbaar. Jullie zijn naar de promotie ook aan een baan op een nieuwe plek begonnen (zelf in een ander land), en binnenkort zal ik eindelijk de tijd hebben om jullie te bezoeken.

Meine unbeschreiblich tollen Eltern: Diana und Papa, ich bin unglaublich froh dass wir so oft telefonieren, ihr mich regelmäßig besucht, und ihr mir immer zur Seite steht wenn ich Hilfe brauche - notfalls auch wenn das mit (spontanen) Reisen verbunden ist. Danke für alles! Meine Grosselten Oma, Rosi und Helmut. Durch meine Promotion im Ausland (bzw. Emigration) sehen wir uns leider viel zu selten. Vielen Dank für euer Verständnis dafür und eure unablässige Unterstützung! Harry, durch dich bin ich früh mit der Wissenschaft in Kontakt gekommen und konnte ich mein erstes Praktikum an der Uni machen, vielen Dank für diese Erfahrung. An den Rest meiner Familie: Vielen Dank für die gute Zeit zuhause und eure bisherigen und hoffentlich zukünftigen Besuche!

Alle Augsburger und Königsbrunner: Wie schön es doch immer ist heimzukommen! Und genauso schön ist es wenn ihr mich hier besucht - vielen Dank für eure Besuche Kathy, Stanczi, Manu und Doro!

Meine Geschwister, Sophia, Jakob und Theresa: Geschwister sind das Tollste was man haben kann! Wir sind alle sehr individuell und eigensinnig, aber ich spüre mit jedem von euch eine so besondere Gemeinsamkeit die man wohl einfach nur mit Geschwistern hat. Ich bin so froh, dass es Menschen gibt die irgendwie so sind wie ich. 


$$
\begin{aligned}
& \text { List of publications } \\
& \text { r!af Ot Dnpl!csf!onz }
\end{aligned}
$$




\section{List of publications}

Hertle E, Arts IC, van der Kallen CJ, Feskens EJ, Schalkwijk CG, Stehouwer CD, van Greevenbroek MM. The alternative complement pathway is longitudinally associated with adverse cardiovascular outcomes. The CODAM study. Thrombosis and Haemostasis, 2015. 115 [doi: 10.1160/th15-05-0439].

Hertle E, Stehouwer CD, van Greevenbroek MM. The complement system in human cardiometabolic disease. Molecular Immunology, 2014. 61: 135-48.

Hertle E, van Greevenbroek MM, Arts IC, van der Kallen CJ, Feskens EJ, Schalkwijk CG, Stehouwer CD. Complement activation products $\mathrm{C} 5 \mathrm{a}$ and SC5b-9 are associated with lowgrade inflammation and endothelial dysfunction, but not with atherosclerosis in a crosssectional analysis: the CODAM study. International Journal of Cardiology, 2014. 174: 400-3.

Hertle E, van Greevenbroek MM, Arts IC, van der Kallen CJ, Geijselaers SL, Feskens EJ, Jansen EH, Schalkwijk CG, Stehouwer CD. Distinct associations of complement C3a and its precursor C3 with atherosclerosis and cardiovascular disease. The CODAM study. Thrombosis and Haemostasis, 2014. 111: 1102-11.

Hertle E, van Greevenbroek MM, Stehouwer CD. Complement C3: an emerging risk factor in cardiometabolic disease. Diabetologia, 2012. 55: 881-4.

Groen BB, Res PT, Pennings B, Hertle E, Senden JM, Saris WH, van Loon LJ. Intragastric protein administration stimulates overnight muscle protein synthesis in elderly men. American Journal of Physiology, Endocrinology and Metabolism, 2012. 302: 52-60. 


\section{Curriculum Vitae

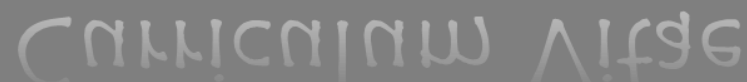




\section{Curriculum Vitae}

Elisabeth Hertle was born on June $4^{\text {th }} 1985$ in Augsburg, Germany. In 2004, she graduated from grammar school (Abitur, Gymnasium Königsbrunn). In 2005, she started her study of Nutritional Science at the Technische Universität München. In 2010, she completed a ninemonth internship at the Department of Human Movement Sciences of Maastricht University and obtained her Master's degree (passed with distinction). From 2011 to 2015, she worked as a PhD researcher at the Department of Internal Medicine of Maastricht University. Concomitantly with her PhD research, she studied Epidemiology in part-time and obtained her Master's degree in 2013 (cum laude). Since January 2016, Elisabeth works as a Pharmacovigilance Manager at the Drug Safety Department of Synthon, Nijmegen. 